University of Louisville

ThinkIR: The University of Louisville's Institutional Repository

8-2015

\title{
Optical and sensing properties of various shaped gold nanoplates and highly controlled asymmetric gold nanoplate/nanosphere coupled assemblies.
}

Aiqin Fang

University of Louisville

Follow this and additional works at: https://ir.library.louisville.edu/etd

Part of the Chemistry Commons

\section{Recommended Citation}

Fang, Aiqin, "Optical and sensing properties of various shaped gold nanoplates and highly controlled asymmetric gold nanoplate/nanosphere coupled assemblies." (2015). Electronic Theses and Dissertations. Paper 2258.

https://doi.org/10.18297/etd/2258

This Doctoral Dissertation is brought to you for free and open access by ThinkIR: The University of Louisville's Institutional Repository. It has been accepted for inclusion in Electronic Theses and Dissertations by an authorized administrator of ThinkIR: The University of Louisville's Institutional Repository. This title appears here courtesy of the author, who has retained all other copyrights. For more information, please contact thinkir@louisville.edu. 
OPTICAL AND SENSING PROPERTIES OF VARIOUS SHAPED GOLD NANOPLATES AND HIGHLY CONTROLLED ASYMMETRIC GOLD NANOPLATE/NANOSPHERE COUPLED ASSEMBLIES

\title{
By
}

\author{
Aiqin Fang \\ A Dissertation \\ Submitted to the Faculty of the \\ College of Arts and Sciences of the University of Louisville \\ in Partial Fulfillment of the Requirements \\ for the Degree of
}

Doctor of Philosophy in Chemistry

Department of Chemistry

University of Louisville

Louisville, Kentucky

August 2015 
Copyright 2015 by Aiqin Fang

All rights reserved 



\title{
OPTICAL AND SENSING PROPERTIES OF VARIOUS SHAPED GOLD NANOPLATES AND HIGHLY CONTROLLED ASYMMETRIC GOLD NANOPLATE/NANOSPHERE COUPLED ASSEMBLIES
}

\author{
By \\ Aiqin Fang \\ A Dissertation Approved on
}

May 13, 2015

by the following Dissertation Committee:

Dissertation Director

Dr. Francis P. Zamborini

Dr. Craig A. Grapperhaus

Dr. Richard P. Baldwin

Dr. Aleeta M. Powe

Dr. Gamini U. Sumanasekera 


\section{DEDICATION}

This dissertation is dedicated to my parents and adoptive father

Shiping Fang and Mingying Chi

Shixing Fang

My wonderful husband and sons

Jubin Chen

Chris Chen and Charles Chen 


\section{ACKNOWLEDGEMENTS}

First of all, I would like to acknowledge my advisor Dr. Francis P. Zamborini without whom none of this would have been possible. I appreciate him for taking me into his group, teaching me to think independently, to do research, and to have a vision for the future. He is always there for scientific discussions and research difficulties. His insight, support, and encouragement during these four years have been invaluable.

Second, I would like to thank my committee members Dr. Craig A. Grapperhaus, Dr. Richard P. Baldwin, Dr. Aleeta M. Powe, and Dr. Gamini U. Sumanasekera for their valuable advice and suggestions on my research. I also would like to thank all current and former group members. Dr. Radhka Dasari and Lanlan Bao have mentored and trained me when I first joined the group. Nidhi Shah and others are always willing to listen to my difficulties, discuss with me, and give me valuable suggestions.

Third, I would like to acknowledge our collaborators Dr. Prashant Jain and Sarah White from University of Illinois at Urbana-Champaign. Without their expertise in electrodynamic simulation of plasmon resonance, it would be very hard for us to interpret some of the experimental results. Dr. Tereza Paronyan from the ElectroOptics Research Institute and Nanotechnology Center deserves special thanks for her assistance with SEM and AFM. I appreciate the help from Dr. Julia Aebersold and Curt McKenna from Micro Nano Technology Center on fabrication of glass slides with etched numbers. A 
deep gratitude goes to all the faculties and staff from Department of Chemistry for serving me in many different ways.

Last but not least, I would like to acknowledge my parents and adoptive father for making many sacrifices in supporting me to pursue my studies thousands of miles away from home. I also would like to express my deep love and appreciations to my husband Jubin Chen for always encouraging and supporting me in the tough times. A special thank to my sweet angels Chris and Charles for bringing so much joy and happiness to my life. 


\begin{abstract}
OPTICAL AND SENSING PROPERTIES OF VARIOUS SHAPED GOLD NANOPLATES AND HIGHLY CONTROLLED ASYMMETRIC GOLD NANOPLATE/NANOSPHERE COUPLED ASSEMBLIES
\end{abstract}

Aiqin Fang

May 13, 2015

With the development of a strategy to correlate the dark-field light scattering spectra of individual nanostructures with scanning electron microscopy (SEM) and atomic force microscopy (AFM) images of the same nanostructures, we were able to investigate several interesting optical properties of $\mathrm{Au}$ nanoplates (NPs) and asymmetrically-coupled Au nanospheres (NSs) attached to Au NPs with a high level of control. The light scattering spectra of the NP/NS coupled structures depend strongly on the location of NS attachment on the NP. Attachment of multiple NSs at the edge/vertex sites leads to a unique synergistic effect. In contrast to the uniform distribution of NSs, asymmetric distributions of multiple NSs attached to the sides of a NP result in complex, broadened, multi-peaked spectra with larger plasmonic shifts. Simulations using the discrete dipole approximation (DDA) method verified all of the experimental results.

The positive shift in the dipolar plasmon mode of the NP/NS assembly relative to the original NP increases with increasing NS size for those attached on the side of the NP 
in the order of $9 \pm 2 \mathrm{~nm}, 24 \pm 4 \mathrm{~nm}$, and $98 \pm 16 \mathrm{~nm}$ for the 13,24 , and $51 \mathrm{~nm}$ average diameter NSs, respectively. For a NS attached to the top terrace of a NP, the shift in the dipolar plasmon mode is $1 \pm 1 \mathrm{~nm}, 3 \pm 1 \mathrm{~nm}$, and $14 \pm 4 \mathrm{~nm}$ for the 13,24 , and $51 \mathrm{~nm} \mathrm{NS}$, respectively, and the spectra become more broad. The attachment of a Au NS to a hexagonal or circular Au NP through a cysteamine (Cys) linker shows different light scattering properties compared to attachment through 4-aminothiophenol (4-ATP). The shorter length of Cys leads to stronger dipolar plasmon coupling along the long axis of the NP/NS structure. This leads to a larger red-shift compared to linking with 4-ATP. The geometric shape of the NPs dramatically affects their sensitivity to refractive index changes in the environment and sensitivity to the attachment of a Au NS. The sensitivity of $\lambda \max$ to a change in the refractive index of the environment followed the order of triangles $>$ hexagons $>$ circles. This research provides new fundamental information and a better understanding of shape-dependent optical properties and plasmon coupling of asymmetric metallic nanostructures with potential use in three-dimensional spatial sensing and other plasmonic applications. 
TABLE OF CONTENTS

PAGE

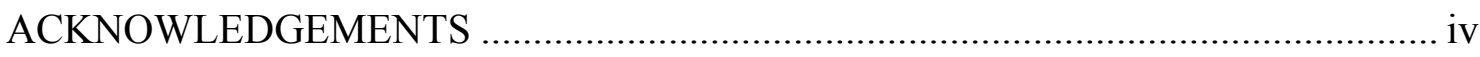

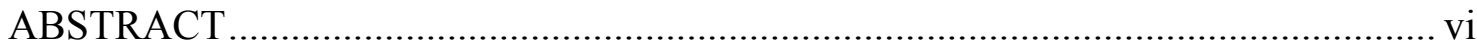

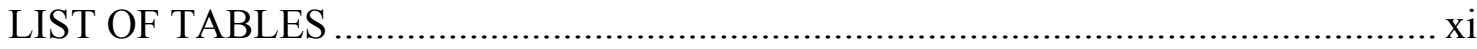

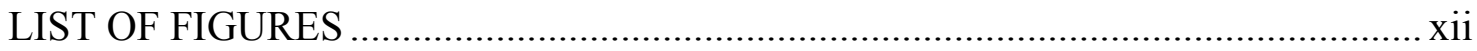

\section{CHAPTER}

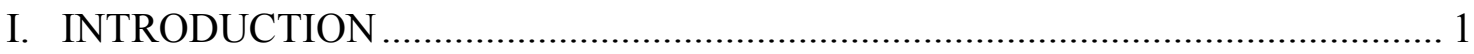

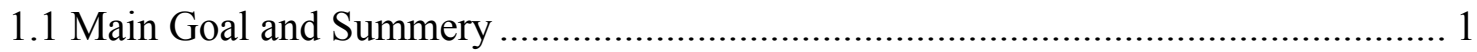

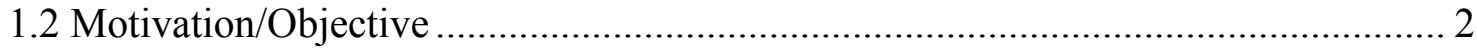

1.3 Synthesis and Assembly of Metallic Nanostructures .............................................. 3

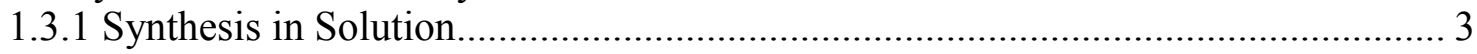

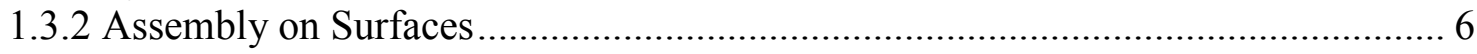

1.3.3 Direct Fabrication and Synthesis on Surfaces ………........................................... 7

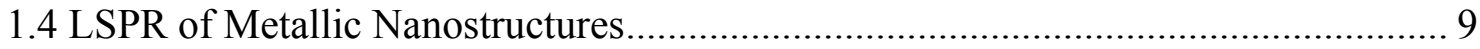

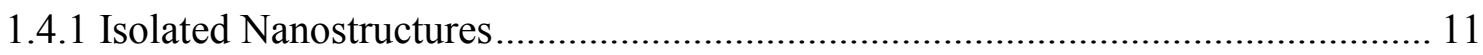

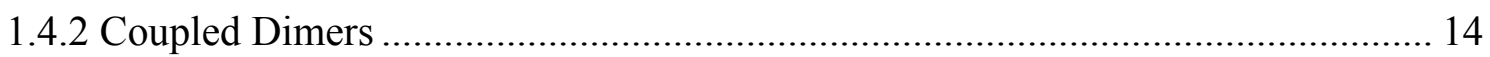

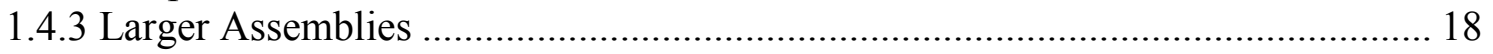

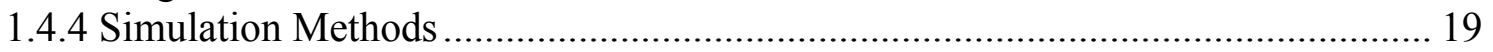

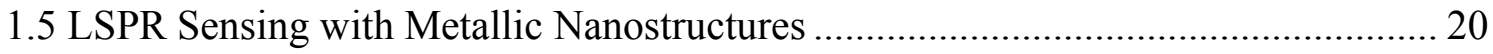

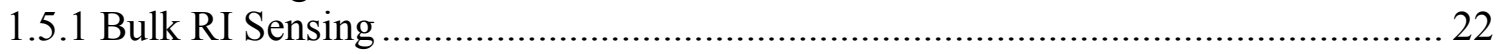

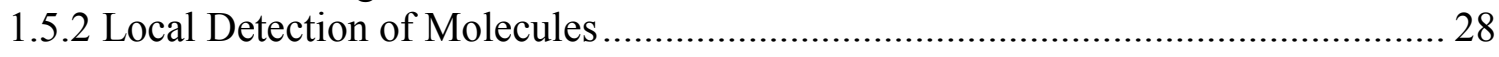

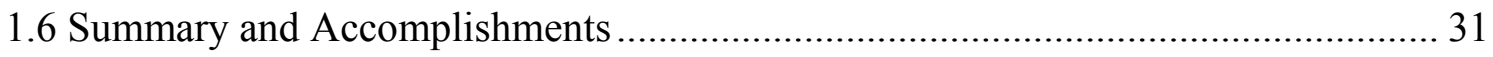

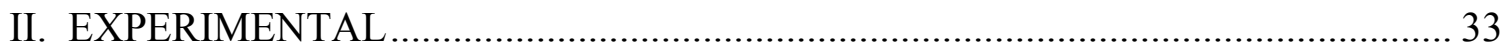

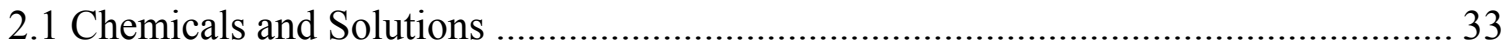

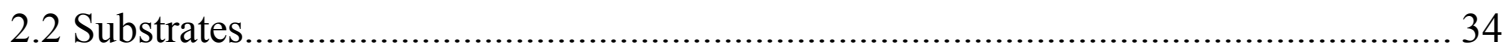

2.3 Synthesis of 3-5 nm Diameter Gold Nanosphere (NS) Solution. ............................... 34

2.4 Preparation of $25 \mathrm{~nm}$ Diameter Citrate-Stabilized Au NSs........................................ 34

2.5 Preparation of $\sim 51 \mathrm{~nm}$ and $\sim 13 \mathrm{~nm}$ Diameter Citrate-Stabilized Au NSs................ 35

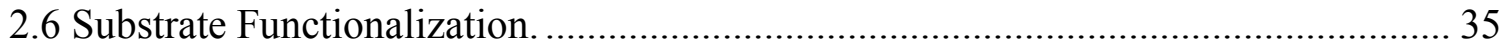

2.7 Synthesis of Au NPs Directly on Glass Surfaces with Optimal Size and Coverage 35

2.7.1 Optimization of Au NP Coverage for Single Particle Dark-Field (DF) Scattering. 38

2.7.2 Optimization of Au NP Size for Single Particle DF Scattering.............................. 40 
2.8. Attachment of $25 \mathrm{~nm} \mathrm{Au} \mathrm{NSs} \mathrm{to} \mathrm{Au} \mathrm{NPs} \mathrm{via} \mathrm{Organic} \mathrm{linkers.................................} 42$

2.8.1 The $\mathrm{pH}$ Value of 4-ATP Monolayer Protonation... ………................................... 42

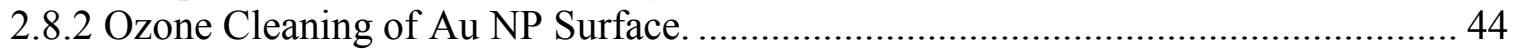

2.9 Controlled Attachment of Au NSs to the Vertex/Edge Sites of Au NPs. .................. 46

2.10 Controlled Attachment of Au NSs to Terrace Sites of Au NPs................................ 48

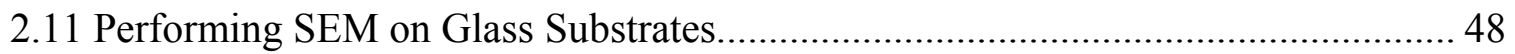

2.12 Correlating SEM with LSPR Spectra...............................................................5 54

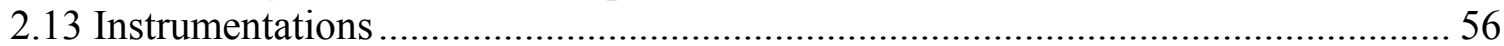

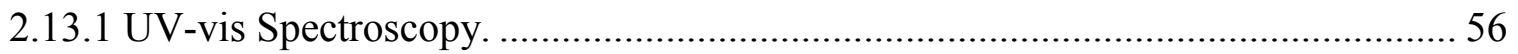

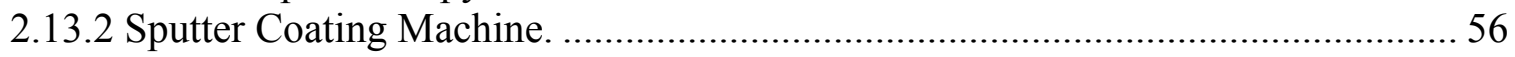

2.13.3 Scanning Electron Microscopy (SEM) …………........................................ 56

2.13.4 Dark-Field Microscopy/Spectroscopy. ........................................................... 57

2.13.5 LSPR Spectrum Acquisition and Process ........................................................... 57

III. REGIOSELECTIVE PLASMONIC COUPLING IN METAMOLECULAR

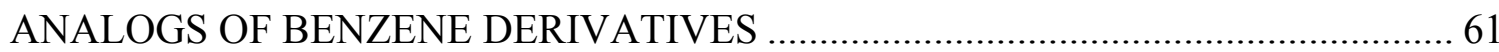

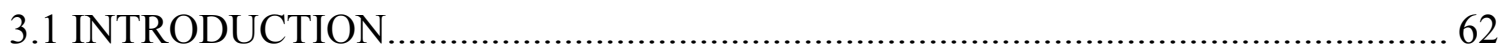

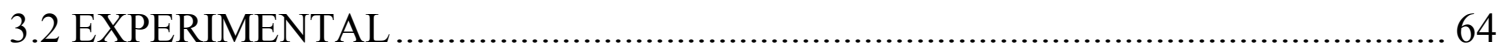

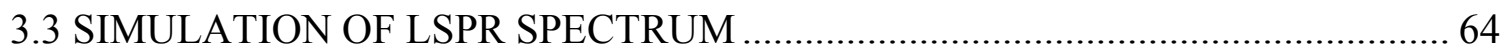

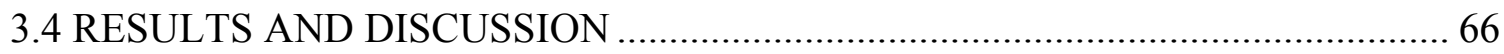

3.4.1 Correlation of LSPR Spectrum with SEM Images. .................................................6 66

3.4.2 Experimental Comparison of Terrace Site vs Edge Site Attachment ....................... 67

3.4.3 Discrete Dipole Approximation (DDA) Simulation.............................................. 74

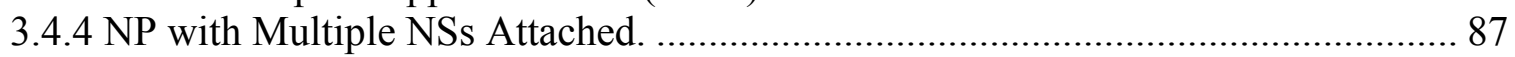

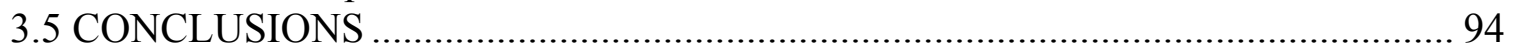

IV. THE EFFECT OF NS SIZE ON THE PLASMONIC PROPERTIES OF REGIOSELECTIVELY-COUPLED GOLD NP/NS STRUCTURES AT THE

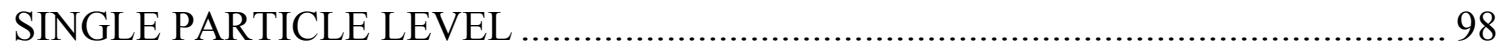

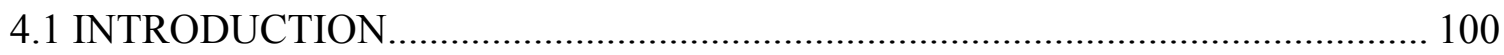

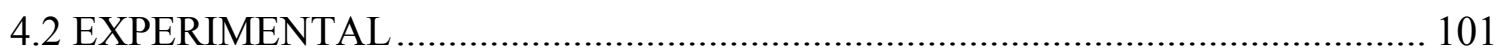

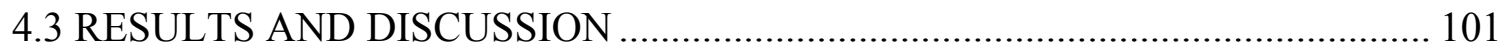

4.3.1 Size-Dependent Plasmon Properties of NP/NS Dimers with NSs Attached to the

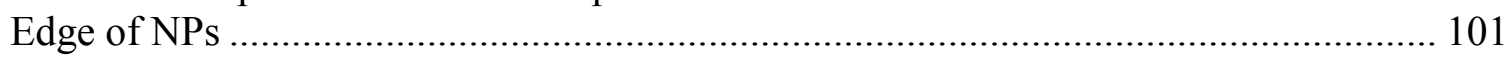

4.3.2 Size-Dependent Plasmon Properties of NP/NS Structures with NSs Coupled to the

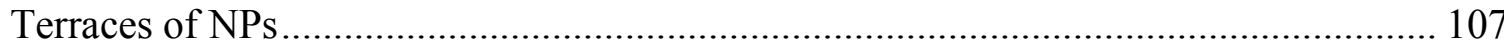

4.3.3 Multiple Big NSs Coupled to the Edge and Terraces of NPs .............................. 112

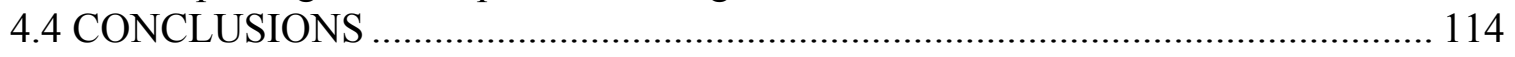

V. THE EFFECT OF LINKER ON THE PLASMONIC COUPLING BETWEEN

GOLD NANOSPHERES AND GOLD NANOPLATES MEASURED AT THE

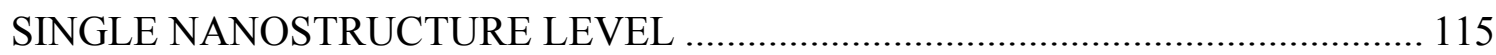

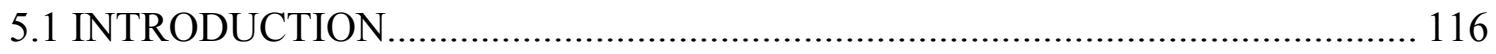

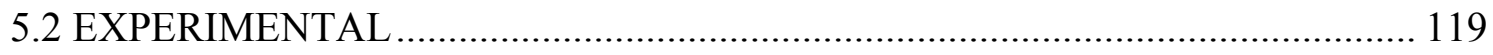

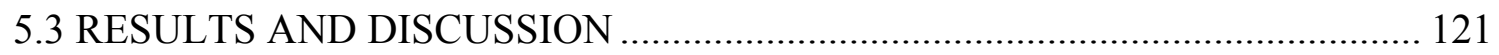


5.3.1 The Coupling of Au NS to Hexagonal Au NP through Cys and 4-ATP Linkers . 121 5.3.2 The Coupling of Au NSs to Circular Au NPs through Cys and 4-ATP Linkers .. 127 5.3.3 The Effect of Coupling Location on the Plasmonic Properties of NSs Attached to Triangular NPs

5.4 CONCLUSIONS

VI. EFFECT OF NP SHAPE ON LIGHT SCATTERING PROPERTIES AND

SENSITIVTY TO CHANGES IN THE REFRACTIVE INDEX OF THE

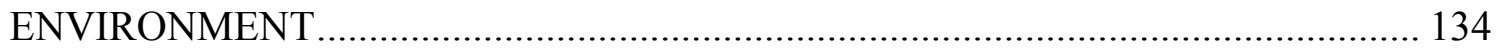

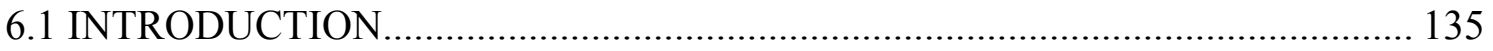

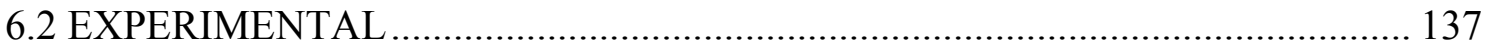

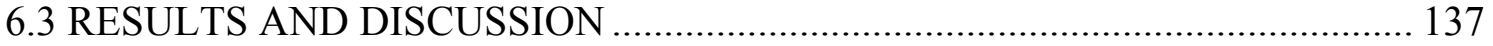

6.3.1 LSPR Correlated to Aspect Ratio of Individual Au NPs .................................... 137

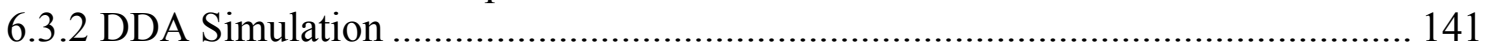

6.3.3 Bulk Refractive Index Sensing of Different Shaped NPs ................................... 142

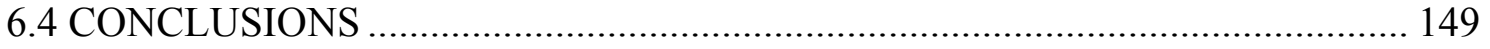

VII. SUMMARY AND FUTURE DIRECTIONS .............................................. 150

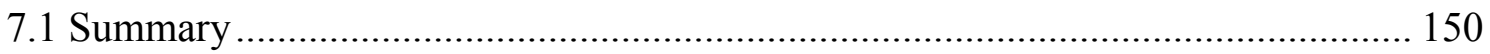

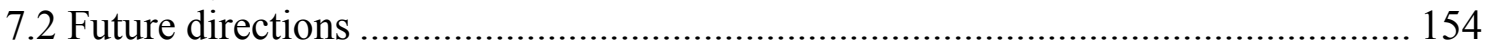

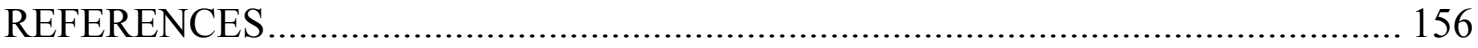

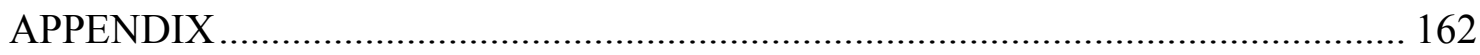

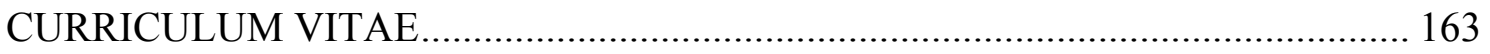




\section{LIST OF TABLES}

TABLE

PAGE

3.1. LSPR data and SEM images of representative single Au NPs with a NS 71 attached on the terrace.

3.2. LSPR data and SEM images of representative single Au NPs with a NS attached on a side face/vertex.

4.1 LSPRs and SEM images of representative single Au NPs with different size 106 NSs attached on the edge sites of the NPs.

4.2. Table 4.2. LSPRs and SEM images of representative single Au NPs with 110 different size NSs attached on the terrace sites of the NPs.

6.1. LSPR $\lambda_{\max }$ shift data and pertinent experimental environmental sensitivity parameters for dipole resonance of three different triangular NPs.

6.2. LSPR $\lambda_{\max }$ shift data and pertinent experimental environmental sensitivity 148 parameters for dipole resonance of four different hexagonal NPs.

6.3. LSPR $\lambda_{\max }$ shift data and pertinent experimental environmental sensitivity 148 parameters for dipole resonance of two different circular NPs. 


\section{LIST OF FIGURES}

$\begin{array}{ll}\text { FIGURE } & \text { PAGE }\end{array}$

1.1. Depiction of the response of a nanoparticle's conduction band electrons under 10 electromagnetic excitation.

1.2. Schematic illustration of symmetric (A) and asymmetric (B) nanoparticle coupling.

1.3. A) Apparatus for measuring dark-field scattering. B) Diagram showing the

conical illumination. Yellow lines represent transmitted light missing the objective and green lines represent the scattered light detected by the objective.

1.4 Dark-field microscopy images of Au nanoparticles on a glass slide at 100x magnification. Scale bar is $10 \mu \mathrm{m}$.

2.1 A schematic illustration of the growth of $\mathrm{Au}$ nanostructures directly on the surface of glass and $\mathrm{Si} / \mathrm{SiOX}$ substrates by the seed-mediated growth procedure.

2.2. Dark-field and SEM images of Au NP samples synthesized from Au seeds solution without dilution (A and $\mathrm{B}$ ) and with 1:10 dilution (C and D). Scale bars in DF images are $40 \mu \mathrm{m}$ and in SEM images are $5 \mu \mathrm{m}$.

2.3. SEM images and scattering spectra of Au NPs samples synthesized in growth solution for 60 min (A and B) and 5 min (C and D). Scale bars in the SEM images are $200 \mathrm{~nm}$. 
2.4. SEM images of NSs attached to a planar Au film surface by soaking in a $\mathrm{Au}$

NS solution at pH 4.5 (A) and 5.5 (B). The scale bars in the SEM images are 200 nm.

2.5. SEM images of NSs attached to 4-ATP-functionalized Au NPs without exposure to ozone (A) and after exposure to ozone for $10 \mathrm{~min}(\mathrm{~B})$. The scale bars in the SEM images are $100 \mathrm{~nm}$.

2.6. A schematic illustration of the attachment of $25 \mathrm{~nm} \mathrm{Au} \mathrm{NSs} \mathrm{onto} \mathrm{the} \mathrm{vertex}$ and edge sites of Au NPs via ligand place-exchange reaction between HT and 4ATP for $1 \mathrm{~h}$.

2.7. A schematic illustration of the attachment of $25 \mathrm{~nm} \mathrm{Au} \mathrm{NSs} \mathrm{onto} \mathrm{the} \mathrm{terrace}$ sites of Au NPs via ligand place-exchange reaction between 4-ATP and HT for 1 h.

2.8. SEM images of Au NPs on silicon substrate (A) and a glass substrate (B). 50 Scale bars in SEM images are $500 \mathrm{~nm}$.

2.9. SEM images of Au NPs on a glass substrate with SEM acceleration voltage at (A) $15 \mathrm{KV}$ and (B) $1 \mathrm{KV}$. Scale bars in SEM images are $500 \mathrm{~nm}$.

2.10. SEM images of Au NPs on glass substrates with a Pt coating by sputtering for (A) $10 \mathrm{~min}$, (B) 5min, (C) $3 \mathrm{~min}$, (D) and (E) 1 min. Scale bars in SEM images $(\mathrm{A}, \mathrm{B}, \mathrm{C})$ are $2 \mu \mathrm{m}$ and in images $(\mathrm{D}, \mathrm{E})$ are $100 \mathrm{~nm}$.

2.11. Dark-field images (A, C) and correlated SEM images (B, D) of Au NPs on a glass substrate correlated by using the etched marker number 223. Scale bars in DF and SEM images are $10 \mu \mathrm{m}$. 
2.12. A) Dark-field image of a selected sample area with four nanoparticles of interest. B) LSPR map of the same area and corresponding nanoparticles.

2.13. A) Raw LSPR spectrum of a single Au nanoplate. B) LSPR spectrum after processing.

3.1. Correlated single-nanoparticle dark-field spectroscopy/ electron microscopy. Dark-field microscopy image of Au NP scatterers on a glass slide in the region of an etched marker labeled '223' (A) and in a region directly to the left of the marker (B). The region marked by the blue rectangle in (B) is shown at a higher magnification in $(C)$ with five NPs selected and numbered. The light-scattering spectrum of the Au NP labeled 2 is shown in (D). The spectrum shows a wavelength maximum, $\lambda_{\max }$, of $668 \mathrm{~nm}$. An SEM image of the same region as (C) is shown in (E). A higher magnification SEM image of the NP labeled 2 is shown in $(\mathrm{F})$.

3.2. Terrace site vs. edge site attachment. (A) Scattering spectrum of a single Au NP before (black line) and after (red line) attachment of a $24 \mathrm{~nm}$ diameter Au NS on an inner terrace site of the NP, as shown in the SEM image to the right. (B) Scattering spectrum of a single Au NP before (black line) and after (red line) attachment of a $27 \mathrm{~nm}$ diameter $\mathrm{Au}$ NS on a vertex site, as shown in the SEM image to the right. The $\lambda \max$ values were determined by fitting the spectra to single Lorentzian peaks. The shift in $\lambda$ max upon NS attachment is indicated.

3.3. Experimental and simulated scattering spectra of a hexagonal Au NP. (A) Experimental scattering spectrum (black curve) of a single hexagonal $\mathrm{Au}$ nanoplate obtained via dark-field spectroscopy and DDA simulated scattering spectrum (red, green, and blue curves). (B) SEM image of the experimental NP. (C) Rendition of the simulated structure.

3.4. Simulated effect of incident light polarization on the plasmon coupling 75 between a Au NP and a Au NS attached to a vertex on the side of the NP.

3.5. Simulated plasmon coupling between a Au NP and a Au NS: effect of the 78 attachment location of the NS along the sides of the NP. 
3.6. Simulated plasmon coupling between a hexagonal Au NP and an Au NS: effect of the location of the NS on the terrace of the NP.

3.7. Simulated distance-dependence of plasmon coupling between a Au NP and a single Au NS for two cases: a NS placed at a vertex on the side (A) and a NS placed at the center of the terrace (B) as shown by the renditions in the insets. The relationship between inter-particle distance and wavelength shift is shown in (C).

3.8. Simulated near-field of a Au NP coupled to a Au NS placed on the terrace of 82 the NP. The images represent a side-view of a cross-section taken along the polarization direction. The near-field is depicted in the form of an amplitude enhancement $(|\mathrm{E}| /|\mathrm{E} 0|)$ on a color scale.

3.9. Terrace site vs. edge site attachment simulation results (A) Scattering spectrum of a single Au NP before (black line) and after (red line) attachment of a $27 \mathrm{~nm}$ diameter Au NS on an inner terrace site of the NP, as shown in the simulated configurations to the right. (B) Scattering spectrum of a single Au NP before (black line) and after (red line) attachment of a $27 \mathrm{~nm}$ diameter Au NS on a vertex site, as shown in the simulated configurations to the right. The $\lambda_{\max }$ values were determined by fitting the spectra to single Lorentzian peaks. The shift in $\lambda \max$ upon NS attachment is indicated.

3.10. Spatial map of the NP polarizability. Simulated near-field over the surface of a Au NP excited by $645 \mathrm{~nm}$ unpolarized light. The near-field is depicted in the form of an amplitude enhancement (|E|/|E0|) on a color scale.

3.11. Experimental (A \& B) and simulated (C \& D) plasmonic coupling between a $\mathrm{Au}$ NP and multiple NSs. Dark-field scattering spectra of Au NPs before (black) and after (red) three (A) and four (B) $\sim 25 \mathrm{~nm}$ diameter Au NSs are attached to the sides of the Au NPs. The SEM images of the corresponding assemblies are shown on the right.Simulations were performed as described in section 3.3. Here, $27 \mathrm{~nm}$ diameter $\mathrm{Au}$ NSs were placed at three (C) or four (D) vertices along the sides of the NP (red data-points connected by cubic splines). Simulated configurations and NS locations are shown for each case and are representative of experimental structures. For attachment of three NSs, the LSPR wavelength maximum was determined by fitting the simulated scattering spectrum to a single Lorentzian peak. For four NSs, the spectrum was fit to two Lorentzian peaks. The red-shift $\Delta \lambda \max$ indicated for each case is relative to the $\lambda_{\max }$ for a NP without an attached 
NS (spectrum shown by black data-points connected by a cubic spline). Unpolarized light was used for excitation in the simulations, similar to the conditions in experiment.

3.12. Experimental (A \& B) and simulated (C \& D) plasmonic coupling between a $\mathrm{Au}$ NP and multiple asymmetrically distributed NSs. Dark-field light-scattering spectra of a roughly hexagonal Au NP before (black) and after (red) attachment of four (A) and five (B) asymmetrically distributed NSs to the sides of the NP. The SEM images of the corresponding assemblies are shown on the right. Simulations were performed as described in the section 3.3. Here, four $27 \mathrm{~nm}$ diameter $\mathrm{Au}$ NSs were placed along the sides of the NP. The simulated configuration, representative of the experimental configuration in (A), is shown besides each simulated spectrum. In (C), the simulated spectrum of the NP/NS assembly (red data-points connected by a cubic spline) under unpolarized light excitation is shown alongside the simulated spectrum of a NP with no NSs attached (black datapoints connected by a cubic spline), also for unpolarized light excitation. In (D), simulated spectra of the NP/NS assembly are shown for light polarized along the NP short-axis (green data-points connected by a cubic spline) and for light polarized along the long-axis (blue data-points connected by cubic spline). The spectrum for long-axis polarization, fit to two Lorentzian peaks, shows a lower energy band centered at $721 \mathrm{~nm}$, red-shifted from the NP LSPR by as much as 79 $\mathrm{nm}$. The spectrum for short-axis polarization, fit to one Lorentzian peak, shows a band centered at $656 \mathrm{~nm}$.

3.13. Single-particle dark-field light-scattering spectra of a roughly hexagonal Au NP before (black) and after (red) attachment of multiple NSs to NP terrace sites. Final morphologies are displayed on the right.

3.14. Simulated effect of the incident light polarization on the plasmon coupling 95 between an Au NP and four NSs. Simulations were performed for a hexagonal Au $\mathrm{NP}$ with a long axis of $137 \mathrm{~nm}$, a thickness of $31 \mathrm{~nm}$, and a 1-nm thick ligand shell of HT and 4-ATP, representative of the experimental structure. Four Au NSs, each with a diameter of $27 \mathrm{~nm}$, were attached to four of the vertices of the NP, (configuration is shown in Figure 3.16). The surface-to-surface distance between the NP and each NS was $1 \mathrm{~nm}$, measured along the long-axis of the hexagon.

3.15. Simulated near-field over the surface of a Au NP coupled to four Au NSs. The near-field is depicted in the form of an amplitude enhancement $(|\mathrm{E}| /|\mathrm{E} 0|)$ on a color scale. Simulations were performed for a hexagonal Au NP with a long axis of $137 \mathrm{~nm}$, a thickness of $31 \mathrm{~nm}$, and a 1-nm thick ligand shell of HT and 4-ATP, representative of the experimental structure. Four Au NSs, each with a diameter of 
$27 \mathrm{~nm}$, were attached to four of the vertices of the NP, as depicted. The surfaceto-surface distance between the NP and each NS was $1 \mathrm{~nm}$, measured along the long-axis of the hexagon. Three different simulations were run: the direction of polarization and wavelength of incident light employed in each simulation are noted for each near-field plot. An electrostatic charge distribution corresponding to each near-field plot is also shown below each plot.

4.1. Dark-field scattering spectra of single NP before (black) and after (red) NS attached on the edge (left) and their SEM images (right). Scale bars in SEM images are $100 \mathrm{~nm}$.

4.2. (A) Simulated scattering spectra for a hexagon NP coupled with a single NS 104 with $67 \mathrm{~nm}$ in diameter under different polarization conditions. (B) The field enhancement at the surface for the nanoassembly, excited with light polarized along the interparticle axis at two different excitation wavelengths.

4.3. Dark-field light scattering spectra of a single NP before (black) and after (red) 108 NS attached on the terrace (left) and their SEM images (right). Scale bars in the SEM images are $100 \mathrm{~nm}$.

4.4 Dipolar plasmon shift as a function of NS size attached to an edge or terrace. NSs attached to the edges are represented by green circles $(13 \mathrm{~nm})$, blue circles $(24$ $\mathrm{nm})$, and red circles $(51 \mathrm{~nm})$; NSs attached to the terrace are represented by green triangles $(13 \mathrm{~nm})$, blue triangles $(25 \mathrm{~nm})$, and red triangles $(42 \mathrm{~nm})$.

4.5. Dark-field scattering spectra of a single nanoplate before (black) and after 113 (red) NS attachment on the edge (A) or terrace (B) and their SEM images to the right. Scale bars in SEM images are $100 \mathrm{~nm}$.

5.1. SEM images of $25 \mathrm{~nm} \mathrm{Au} \mathrm{NSs} \mathrm{attached} \mathrm{onto} \mathrm{a} \mathrm{Au} \mathrm{NP} \mathrm{under} \mathrm{different}$ 120 conditions. (A) $6 \mathrm{mM}$ Cys overnight and 30 min soaking in a Au NS solution. (B) $6 \mathrm{mM}$ Cys overnight and $5 \mathrm{~min}$ soaking in a Au NS solution. (C) HT-Cys ligand place-exchange for $1 \mathrm{~h}$ followed by 30 min of soaking in a Au NS solution. (D) HT-Cys ligand place-exchange for $1 \mathrm{~h}$ followed by 5 min of soaking in a Au NS solution.

5.2. Scattering spectrum of a hexagonal Au NP before (black line) and after (red line) attachment of a Au NS on an vertex site of the NP vis a (A) Cys linker and (B) 4-ATP linker. The SEM image of the exact structure is shown to the right of 
the spectra. Scale bars in SEM images are $100 \mathrm{~nm}$.

5.3. Illustration of the interparticle distance between a NS and NP linked by (A)

Cys molecule and (B) 4-ATP molecule.

5.4. Dark-field light scattering spectrum of an approximately hexagonal Au NP before (black line) and after (red line) attachment of (A) two Au NSs to the edge site via Cys linkers and (B) three Au NSs on the edge site via 4-ATP linkers. The SEM images show the exact structures used to obtain the data. Scale bars in SEM images are $100 \mathrm{~nm}$.

5.5. Dark-field light scattering spectrum of a circular Au NP before (black line) 129 and after (red line) attachment of a Au NS on the edge site of the NP vis (A) Cys linkers and (B) 4-ATP linkers. The SEM images of the exact structure studied are shown to the right of the spectra. Scale bars in SEM images are $100 \mathrm{~nm}$.

5.6. Dark-field light scattering spectrum of a triangular Au NP before (black line) and after (red line) attachment of a Au NS on a (A) vertex site and (B) the edge site of the NP via Cys linkers. The SEM images to the right show the actual coupled nanostructures. Scale bars in SEM images are $100 \mathrm{~nm}$.

6.1. Dark-field light scattering spectrum (left panel), SEM image (middle panel), and cross-section AFM image (right panel) of an individual triangular Au NP (A), hexagonal NP (B), and circular NP (C). The scale bar in the SEM image is 100 nm.

6.2. Relationship between the LSPR dipole peak position ( $\lambda \max )$ and aspect ratio 139 of triangular (red triangles), hexagonal (green squares), and circular (black circles) NPs.

6.3. LSPR scattering spectrum of a single triangular Au nanoplate obtained via 143 dark-field spectroscopy (black curve) and its DDA simulated scattering spectrum (red data points). SEM image (upper right) and simulated structure (bottom right) are also provided. 
6.4. LSPR scattering spectrum of a single hexagonal Au nanoplate obtained via dark-field spectroscopy (black curve) and its DDA simulated scattering spectrum (red data points). The SEM image (upper right) and simulated structure (bottom right) are also provided to the right.

6.5. Correlated single-NP dark-field spectroscopy/electron microscopy. (A) Dark- 146 field microscopy image of 6 individual $\mathrm{Au}$ nanostructures on a glass slide in air. (B) Dark-field microscopy image of the same 6 individual Au nanostructures on the same glass slide in IPA. (C) The light scattering spectrum of the Au NP pointed out by the white arrows in (A) and (B). (D) An SEM image of the NP pointed by the white arrows in (A) and (B). 


\section{CHAPTER I}

\section{INTRODUCTION}

\subsection{Main Goal and Summary}

The main goal of this research was to study the plasmonic coupling of $\mathrm{Au}$ nanospheres (NSs) with Au nanoplates (NPs) and the factors that can affect this coupling, including attachment location of the NS on the NP, the size of the NS, and the organic ligands linking the NS and NP. The application of this study to refractive index (RI) sensing with different shapes of NPs has also been investigated, including triangular, hexagonal, and circular shapes. Chapter I describes the background information about all the topics related to this research, including the overview of plasmonic nanostructure fabrication and synthesis, introduction to localized surface plasmon resonance (LSPR) of metallic nanostructures, and applications of LSPR sensing. Chapter II describes the chemicals, the experimental procedures, and the instrumentation that were used in this research. Chapter III contains the results and discussion of the effect of NS location on LSPR shift when attached to a NP. Chapter IV shows the effect of NS size on the LSPR shift when coupled with a NP. Chapter V investigates the effect of the organic ligand used to link the Au NS to a NP on the LSPR spectral pattern and shift. Chapter VI compares the sensitivity of different shaped NPs, including triangular, hexagonal, and circular, for RI sensing using LSPR spectroscopy. Chapter VII provides conclusions and future directions of this research. 


\subsection{Motivation/Objective}

The motivation behind this research was to better understand the optical and sensing properties of Au NPs and Au NS/NP assemblies at the single nanostructure level. Initially, our group attached proteins to the edges and terraces of NPs for enhanced LSPR biosensing. The LSPR spectra were collected for ensemble nanoparticles and therefore it was difficult to understand the behavior of single nanoparticles. Our group also used that same strategy to attach $\mathrm{Au}$ NSs to the edges and terraces of Au NPs. Strong coupling between linked nanoparticles can be used to enhanced LSPR sensing and SERS detection. These studies were also performed on ensemble particles. The enhancement was regarded as a result of the collective behavior of all measured nanoparticles. To better understand the effect of location on the sensing and spectral properties, we needed to make measurements at the single particle level. We started with Au NSs coupled to NPs because metal nanoparticles are easier to image compared to proteins. Most important, coupled metal nanostructures are currently of great interest in SERS and LSPR enhancement, light wave-guiding, molecular rulers, and other interesting plasmon phenomena and applications.

Another motivation for this study was to better understand the fundamental optical properties of asymmetric coupled nanostructures. Compared to symmetric coupled nanostructures, asymmetric ones often display new coupling modes and unique spectral features that are not observed with symmetric ones, including symmetry breaking, appearance of otherwise dark out-of-phase hybrid modes, avoided crossing behavior, Fano resonances, and asymmetric scattering properties. ${ }^{1,2}$ Our NS/NP 
nanoassemblies might provide some new and unique optical properties because the coupled nanoparticles are different in both size and shape (a small sphere coupled to a bigger plate), which has been studied for the first time.

\subsection{Synthesis and Assembly of Metallic Nanostructures}

The synthesis of metal nanostructures has been an active area of research due to its wide applications in many fields including photonics, ${ }^{3,4}$ electronics, ${ }^{5,6}$ sensing, ${ }^{7,8}$ and medical imaging. ${ }^{9,10}$ One desirable feature of metal nanostructures for applications is the ability to tune their properties by controlling their size, shape and composition. ${ }^{11-13} \mathrm{~A}$ numbers of synthetic methodologies have been developed to produce isotropic or anisotropic nanostructures with controlled size and shape for a variety of materials. ${ }^{14,15}$ Nanostructures are typically formed by either "top-down" or "bottom-up" approaches. Top-down techniques are basically lithographic techniques to fabricate nanostructures, whereas bottom-up methods involve the reactions of atoms, ions, or molecules to "grow" nanostructures either on substrates or in solution.

\subsubsection{Synthesis in Solution}

Bottom-up wet chemistry synthesis is a widely used approach to make metal nanoparticles with control over their shape, size, composition, and functionality. In this approach, the reduction of metal salts usually occurs in a solution containing a colloidal stabilizer, which can suppress the aggregation of the nanoparticles and control the growth of nanostructures. Ligands, surfactants, or polymers can serve as stabilizers and adsorb to the surface of the nanoparticles to inhibit the aggregation by electrostatic repulsion or 
steric hindrance. In some cases, the stabilizer can also play a role in directing nanoparticle growth to a desired shape. The metal salt reduction occurs upon addition of chemical reductants such as sodium citrate, sodium borohydride, and alcohols. Nanoparticle size and shape can be controlled by the choice of stabilizer, reductant, and relative concentration of the reactants in the oxidation-reduction reaction.

Citrate reduction: Citrate is a relatively mild reducing reagent, which can serve as both a reducing agent and an electrostatic stabilizer in synthesizing Au nanoparticles at elevated temperature. In this protocol, $\mathrm{HAuCl}_{4}$ and $\mathrm{AgNO}_{3}$ are the most commonly used precursor for synthesis of $\mathrm{Au}$ or Ag nanoparticles. When precursors are mixed with citrate reducing reagent, such as trisodium citrate, at boiling temperature in water, elemental $\mathrm{Au}$ or $\mathrm{Ag}$ nucleates and grows to form $\mathrm{Au}$ or Ag nanoparticles. The size of the nanoparticles can be well-controlled by adjusting the citrate to precursors ratio. ${ }^{16}$ It has also been reported that the shape of the nanoparticles can be controlled by changing the $\mathrm{pH}$ value of the solution due to the different protonation states of citrate ions. ${ }^{17}$ However, the mechanism of the citrate reduction reaction has not been well studied. Employing citrate as a reducing agent to make Au nanoparticles was first reported by Turkevitch et al. in $1951^{18}$ and was adopted to synthesize other metal nanostructures. Today, the primary use of the citrate synthesis is in producing large quantities of Au colloids for commercial applications and research.

Seed-mediated growth: Well-defined nanoparticles can also be synthesized by seed mediated growth as reported by the Murphy group in 2001. ${ }^{19}$ In seed-mediated growth, metal nanoparticle seeds are synthesized. Then a certain amount of seeds are added to a pre-prepared growth solution containing precursor metal ions, a mild reducing agent (ascorbic acid), surfactant organic molecules (cetyltrimethylammonium bromide, 
CTAB), and some other necessary additives. After the addition of seeds, the metal ions in growth solution are reduced by reducing agent and directly deposited onto the surface of the seeds, which act as the nucleation sites. In the growth process, surfactants preferably adsorb to a certain facet of the small growing metal crystals and block growth in this direction while allowing the growth in other directions. This anisotropic growth approach leads to the synthesis of anisotropic particles. It is an excellent method for synthesizing nanoparticles with aspect ratios bigger than 1, such as nanorods (NRs), nanowires, and NPs. ${ }^{19-21}$ The nanoparticle shape and aspect ratio can be controlled by the composition and concentration of the surfactant, seeds, metal ions, and reducing agent. One big advantage of the seeding growth method is that it can be used to grow nanoparticles directly onto solid surfaces such as silicon and glass, which is very convenient for microscopy studies. ${ }^{22,23}$

Polyol process: The polyol process represents a robust and versatile synthetic procedure that can be used to generate metal and alloy nanoparticles with a wide variety of sizes and shapes. In a typical synthesis, a polyol (ethylene glycol) serves as both a solvent and reducing agent at elevated temperatures. Capping agents and Au precursors react with preheated polyol solution, which ignites the nucleation process and the growth of nanoparticles. The mechanism in the polyol process has been studied in detail. ${ }^{24}$ Polyol syntheses have been used to produce a variety of nanostructures such as cubes, rods, wires, or spheres by adjusting the relative amounts of the capping agent and the metal precursor ions in solution. ${ }^{25-27}$ Other nanostructures such as NPs, nanobelts, and hollow or alloy nanoparticles were also synthesized by using polyol methods. ${ }^{28-30}$ 


\subsubsection{Assembly on Surfaces}

One approach to assemble nanoparticles on substrates is to synthesize nanoparticles first in solution and then immobilized them to solid surfaces by either simple drop casting or linking. Au nanoparticle films formed in this way have been reported as a good platform to monitor elemental mercury. ${ }^{31}$ Au nanoparticles were first synthesized in solution, then assembled as a 2-D sheet using the Langmuir-Blodgett method discussed as interface-assisted assembly, and finally transferred to solid substrates. Thermally annealed multilayer Au nanoparticle films were also prepared from Au nanoparticles synthesized in solution. ${ }^{32}$ Instead of using interface-assisted assembly, multilayer gold nanoparticle films were formed on substrates using layer-by-layer thermal annealing techniques. The sensing platform built from multilayer $\mathrm{Au}$ nanoparticle films reached picomolar detection limits for the LSPR sensing of lysozyme in a buffer solution. Organic molecules such as amine- or mercapto-terminated silanes were also widely used to immobilize Au or Ag nanoparticles to oxide-bearing substrates such as silica. ${ }^{33,34}$ In this protocol, substrates were first functionalized by organic molecules and then immersed into the nanoparticle solution for a certain time. With the help of organic molecules, nanoparticles were attached to the solid substrate. The LSPR sensing is usually measured by UV-Vis or dark-field spectrometers, which require the substrates to be transparent, such as glass. For example, an optically transparent substrate with $\mathrm{Au}$ nanoparticles has been used to study biomolecular interactions in real time by UV-Vis spectroscopy. ${ }^{35}$ 


\subsubsection{Direct Fabrication and Syntheses on Surfaces}

Lithography fabrication: Top-down lithography nanofabrication offers precise control over nanoparticle shape, size, and inter-particle distance. The lithography methods can be categorized into traditional ones and untraditional ones. Traditional ones include photolithography, ${ }^{36}$ electron beam lithography (EBL), ${ }^{37-39}$ and focused ion beam lithography (FIB). ${ }^{40}$ Photolithography, also named optical lithography or UV lithography, is a massively parallel approach for patterning nanostructures onto solid surfaces. It uses light to transfer a designed nanostructure pattern through a photomask onto a light-sensitive photoresist on the solid substrate. Photolithography techniques are widely used in nanostructure fabrication due to its high productivity and relative low cost as compared to the other two methods. However, its resolution is restricted by the diffraction limit of light used in the photoresist exposure step. Instead of using light to expose a photoresist, EBL and FIB employ an electron beam or ion beam to transfer a designed pattern onto substrates. Greater resolution is achieved in EBL and FIB than in photolithography. The disadvantage is the high cost associated with these processes and the low throughput, which encouraged the development of alternative lithographic techniques. To meet this need, the Van Duyne lab developed two untraditional lithography approaches termed film over NS (FON) ${ }^{41}$ and NS lithography (NSL). ${ }^{42}$ Both of these approaches involves drop coating an aqueous suspensions of polystyrene or silica spheres onto a hydrophilic substrate surface. And then different metals are deposited onto these "sphere masks" by either thermal or electron beam evaporation to form the metallic substrate. It is important to note that lithography techniques are capable of precise control over the size, shape, and spacing of metallic nanostructures. However, it is relatively expensive and also difficult to approach interparticle distances that are 
smaller than $1 \mathrm{~nm} \cdot{ }^{43}$ For periodic arrays of nanoparticles fabricated by EBL, the gap size has a limitation of about $50 \mathrm{~nm}$. FIB milling can produce nanoparticle arrays with a gap as narrow as $20 \mathrm{~nm}^{44}$ A modified version of NSL, called angle-resolved NSL, was reported to produce NSs with gaps as small as $4 \mathrm{~nm}^{45}$ More recently, some other nanofabrication techniques, such as laser printing, ${ }^{46,47}$ nanoimprinting, ${ }^{48}$ and dip-pen nanolithography ${ }^{49}$ have also been used in making nanoparticle assemblies.

Direct chemical growth: A more convenient way to produce nanoparticles on solid substrates is to grow them directly on substrates, thereby eliminating the immobilization procedures. Seed-mediated growth procedures have been used to synthesize a variety of nanostructures directly on substrates. It was first developed by Murphy and coworkers for the preparation of Au NRs in solution. ${ }^{19}$ Later, Markovich and co-workers modified the procedures to successfully grow NRs directly on a mica surface. ${ }^{50} \mathrm{Au}$ NS and NRs were directly growth onto indium tin oxide (ITO) electrode surface by a seed mediated growth process. ${ }^{51}$ In our group, we used seed mediate growth to synthesize $\mathrm{Au} \mathrm{NR/single-wall} \mathrm{carbon} \mathrm{nanotube} \mathrm{(} \mathrm{SWCN}$ ) heterojunctions on silicon substrates. $^{22}$ The optical properties have also been studied by UV-Vis spectroscopy. Recently, our group has developed procedures for synthesizing NPs directly on glass and silicon substrates and explored their biosensing applications, such as monitoring the LSPR shifts caused by the binding of anti-immunoglobulin G to NP surfaces. ${ }^{21,52,53}$ The advantage of growing nanostructures directly on substrates lies in its convenience for following spectrometric measurements and great potential for "on-chip" sensing applications. 


\subsection{LSPR of Metallic Nanostructures}

A plasmon is a collective oscillation of the conducting electrons in a noble metal. These plasmon oscillations can be regarded as mechanical oscillations of the free electron "gas" of a metal. In the presence of an external electric field, the electron gas is delocalized with respect to the imaginary fixed positive cores. Plasmons take the form of surface plasmons (SPs) at the surface of a metal such as Ag and Au. SPs can be excited by incident light, which produces an oscillating electric field on the metal surface. When the wavelength of the incident light matches the frequency of plasmon oscillations, a phenomenon called surface plasmon resonance (SPR) occurs. SPR can be divided into two types based on the supporting plasmonic metal structures: propagating surface plasmon resonance (PSPR) and localized surface plasmon resonance (LSPR). PSPR is supported by structures that have at least one dimension that approaches the excitation wavelength and other dimension much larger, such as a thin metal film or long metal wire. In contrast, LSPR is generated from nanostructures with dimensions much smaller than the wavelength of light in all dimensions, such as with metal nanoparticles (Figure 1.1). LSPR has two important effects. First, electric fields near the surface of the metal nanoparticle are greatly enhanced; this enhancement decays rapidly as a function of distance from the particle surface. Second, the extinction or scattering spectrum of metal nanoparticles has a maximum intensity at the wavelength of the plasmon resonance $\left(\lambda_{\max }\right)$. This $\lambda_{\max }$ depends on the size, shape, and composition of the nanoparticles. It also depends on the RI of the surrounding dielectric environment, which is the basis for LSPR sensing.

To understand in depth how the LSPR phenomenon arises, we must turn to the physics of light scattering. The scattering of light is evident in our daily life as it is 


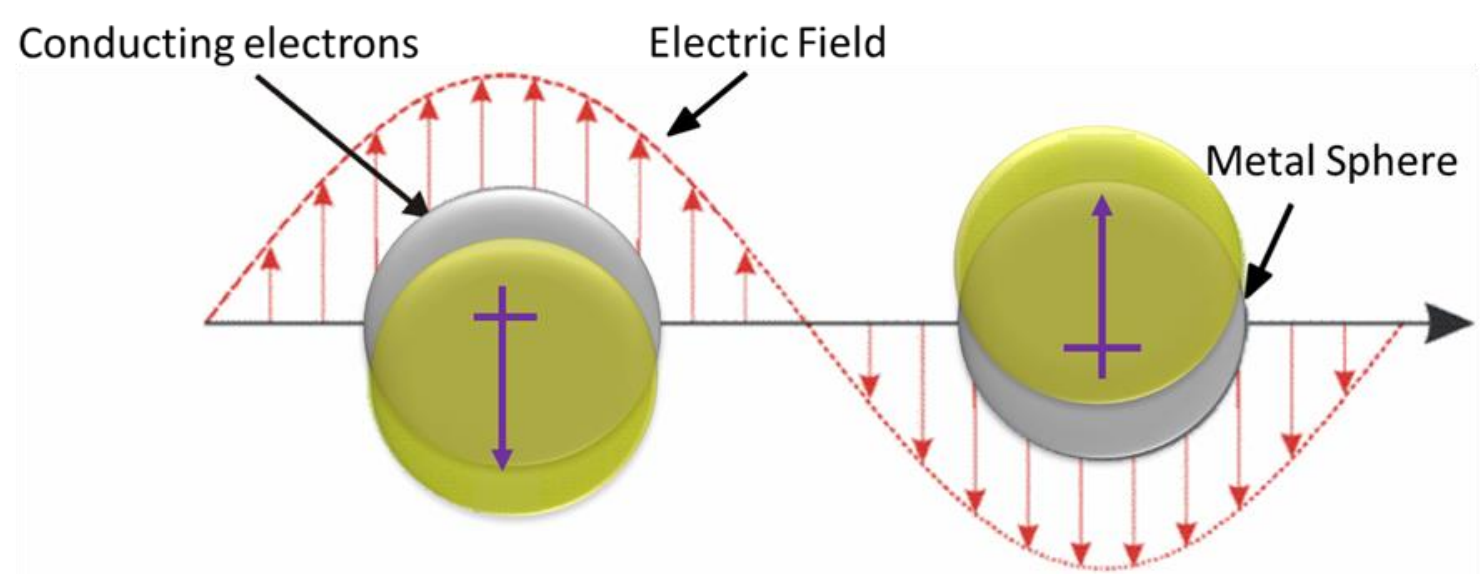

Figure 1.1 Depiction of the response of a nanoparticle's conduction band electrons under electromagnetic excitation. 
responsible for the colorful blue sky and white clouds. In the early 20 th century, Gustav Mie developed a general method to calculate the intensity of light scattered by spherical particles by solving Maxwell equations. Maxwell's equations are a set of partial differential equations which describe how electric and magnetic fields are generated and altered by each other and by currents and charges. When the particles are very small (the radii of particles $<<$ the wavelength of the scattered light), Mie scattering agrees with Rayleigh scattering, which is elastic scattering. When the radii of the particles are comparable or even larger than the wavelength of incident light, the diffraction theory of classical optics will apply. The LSPR wavelength is described by an equation derived from Mie theory: ${ }^{54,55}$

$$
E(\lambda)=\frac{24 \pi^{2} N a^{3} \varepsilon_{\text {out }}^{3 / 2}}{\lambda \ln (10)}\left[\frac{\varepsilon_{\text {i }}(\lambda)}{\left(\varepsilon_{r}(\lambda)+x \varepsilon_{\text {out }}\right)^{2}+\varepsilon_{\mathrm{i}}(\lambda)^{2}}\right]
$$

Where $\mathrm{E}(\lambda)$ is the EM field magnitude. $\mathrm{N}$ is the number of nanoparticles excited by the incident light. $\mathrm{a}$ is the radius of the metallic nanoparticle. $\varepsilon_{\text {out }}$ is the dielectric constant of the medium surrounding the nanoparticle. $\lambda$ is the wavelength of the incident light. $\varepsilon_{\mathrm{r}}(\lambda)$ and $\varepsilon_{i}(\lambda)$ are the real and imaginary portion of the metal dielectric function, respectively. The $\chi$ term is a term determined by nanoparticle shape. It is 2 for a spherical nanoparticle. This term increases in value for nanoparticles of increasing aspect ratio. ${ }^{56,57}$ The $\lambda_{\max }$ and absorbance/scattering intensity of metal nanoparticles are sensitive to changes in the dielectric constant of the surrounding medium. This equation provides theoretical explanations for LSPR sensing.

\subsubsection{Isolated Nanostructures}


Different shape nanoparticles: Nanoparticles can be different shapes. The effect of shape is often stated as significant for spectral properties and LSPR sensitivity of nanoparticles. A number of studies have been conducted to synthesize nanoparticles with different shapes including NSs, NRs, cubes, plates, bipyramids, and nanostars (nanobranches). Spherical nanoparticles are the most simple and widely used plasmonic nanostructures. Wet chemistry synthesis tends to yield approximately spherical particles since the lowest surface energy shape is that of a sphere. Spherical nanoparticles are versatile building blocks for construction of other complex nanostructures such as dimer, trimer, oligomer, or core-satellites clusters. However, the aspect ratio of a perfect sphere is 1 , which restricts its application in exploring the polarization dependence of incident light. On the contrary, NRs are excellent candidates for studying the optical properties related to aspect ratio of nanostructures. Gold NR extinction spectra have two plasmon modes, which correspond to plasmon resonance along their length (longitudinal resonance) and across their diameter (transverse resonance) ${ }^{58}$ Control over the aspect ratio of NRs can be easily achieved either using lithographic techniques or wet chemistry methods. It has been reported that the maximum wavelength of absorption of NRs increases with increasing aspect ratio. NSs and NRs are good shapes for studying fundamental properties of nanoparticles. When it comes to applications such as enhancement of SERS signal or LSPR sensitivity, nanostructures with sharp corners are desired due to very strong electric field enhancement at those locations. Previous studies showed that the electromagnetic field was more intense at the sharp corners of several nanostructures such as cubes, plates, and bipyramids. More recently, nanostars became the real star in the nanoparticle world due to its high sensitivity for chemical detection and medical applications. ${ }^{59-62}$ 
Optical properties related to shape: The specific geometry of a nanoparticle plays a major role in determining the plasmon resonance frequency and number of plasmon modes observed. Several general phenomena can be observed among the nanoparticles. First of all, the number of plasmon modes increases as the symmetry of a nanoparticle decreases. For example, the spectrum of a spherical nanoparticle has only one strong dipole mode while the spectrum of a NR possesses two strong modes including the longitudinal and transverse resonance. ${ }^{25,63}$ Compared to a sphere, a rod has less symmetry, leading to different polarization in different directions. A similar trend has also been observed in the spectra of a bipyramid and octahedral nanoparticle. ${ }^{12}$ Multilploar modes appear in addition to dipole excitation. Secondly, the position of the plasmon resonance can also be tuned by changing the shape of the nanoparticles. For a NS and a nanocube of similar size, the dipole peak of a nanocube is red-shifted $100 \mathrm{~nm}$ relative to that of the sphere. Schultz and coworkers reported a study of shape effects in the plasmon resonance of Ag nanoparticles. ${ }^{64}$ In their work, they observed the dipole peak blue shifting when a triangular NP lost its sharp corners by heating. The sharp corners of the nanoparticles allows for greater charge separation during the dipole oscillation, producing a reduced restoring force and the result is a longer resonance wavelength. ${ }^{65}$ Finally, nanoparticle shape can also affect the intensity of scattering and absorption. Strong scattering and absorption are produced by the formation of a strong dipole on the nanoparticles which completely depends on the nanoparticle symmetry. For nanoparticles with centrosymmetric shapes such as spheres, cubes, and octahedrons, a strong dipole can be easily formed from the charges concentrating on the opposite side of the nanostructures. However, other factors like size may play a more prominent role 
in the magnitude of the scattering and absorption cross sections of a nanoparticle instead of geometry.

\subsubsection{Coupled Dimers}

One of the main driving forces pushing the development of synthetic methodologies for assembling nanostructures is the unique optical properties of coupled nanostructures. Electromagnetic field enhancement at the junction of the coupled nanoparticles is one of the most important and widely applied properties. The applications include optical sensing, ${ }^{66-69}$ single-molecule detection by surface-enhanced Raman spectroscopy (SERS), ${ }^{70-74}$ and wave-guiding of light. ${ }^{75,76}$ Even the most simple coupled nanostructure, NS dimers, have very unique optical properties due to the strong surface plasmon coupling. As a byproduct of nanoparticle assembly, the junctions between two adjacent metallic nanoparticles can concentrate light and result in highly intense electromagnetic fields. Electrodynamic calculations show the intensity of the junction depends strongly on the polarization direction of the incident light. To understand in depth how two nanoparticles couple, we have to discuss plamson hybridization theory.

Plasmon hybridization theory: The simplest description of the interaction between two adjacent nanoparticles is the coupling of two nearby dipoles. The interaction energy is determined by the magnitudes of the dipole moments and the interparticle distance. ${ }^{77,78}$ The coupling energy is strong enough for two close metal nanoparticles to affect their original resonance frequencies, resulting in red and/or blue shifts in the LSPR frequency. Two dipoles of interacting metal nanoparticles can be 
represented by two orbitals of an atom. The plasmonic coupling of two nanoparticles can be viewed as hybridization of two orbitals. Plasmonic modes generated from coupled nanoparticles are analogous to hybrid orbitals formed from the hybridization of atomic orbitals upon bonding. This analogy provides a simple way to understand how plasmon oscillations from individual nanoparticles interact in a coupled nanostructure. Plasmon coupling models have been established based on electrodynamic theory and compared to experimental spectra of coupled nanostrucutures. In the work of several groups, orbital hybridization theory has been used to simulate plasmon coupling. ${ }^{79,80}$

The dimer is the simplest configuration of two coupled metal nanoparticles, which can be formed from those with identical (homodimer) or different (heterodimer) size, shape, or composition. In the case of a NS homodimer as shown in Figure 1.1A, the plasmon resonances of the two interacting metal spheres can hybridize either in-phase (symmetric) or out-of-phase (antisymmetric). When the polarization direction of incident light is parallel to the interparticle axis (longitudinal polarization), the in-phase hybridization produces a lower-energy LSPR band ( $\sigma$ type) which gives rise to an enhanced electric field and a red-shifted plasmon frequency. The out-of-phase coupling of dipoles ( $\sigma^{*}$ type $)$ is spectrally dark due to a zero net dipole moment resulting from the cancellation of the equal but oppositely oriented dipoles on the two coupled nanoparticles. However, when the polarization direction is perpendicular to the interparticle axis (transverse polarization), the in-phase coupling produces higher-energy LSPR band ( $\pi^{*}$ type), and the out-of-phase combination ( $\pi$ type) is spectrally dark due to the cancellation of dipoles. Hence, only head-to-tail $(\sigma)$ coupling under longitudinal polarization and side-by-side $\left(\pi^{*}\right)$ coupling under transverse polarization are observed in the LSPR spectra which is represented by a large red-shifted frequency and relatively 
small blue-shifted frequency, respectively. These interactions are known as dipolar coupling. Higher order multipoles can also be observed in the case of large or more complex coupled nanostructures. When it comes to NS heterodimers (Figure 1.2 B), the coupling is more complicated since the coupling of heterodimers breaks the symmetry and results in incomplete cancellation of oppositely oriented dipoles on the two particles in out-of-phase modes $\left(\sigma^{*}\right.$ and $\pi$ ). They are not spectrally dark any more. For scattering of unpolarized light, four modes should be observed with varied intensity, theoretically. However, out-of-phase modes are often too weak to be observed in experiments.

Symmetric coupled nanostructures: The optical properties of various homodimers, such as NS-NS nanoshell-nanoshell, and nanocube-nanocube pairs, have been previously simulated and, in some cases, compared to experimental observations. ${ }^{81-}$ 84 Jain et al. fabricated various nanodisc homodimer pairs with controlled spacing and orientation by electron beam lithography (EBL), allowing them to derive a plasmon ruler equation to estimate the distance between particles based on the shift in the plasmon band. ${ }^{85}$ The optical properties of Au NR (NR)-Au NR homodimers, arranged side-toside, end-to-end, or perpendicular to one another has been studied experimentally and theoretically, showing a large dependence on the specific arrangement. ${ }^{80,86}$ Yang and coworkers used EBL to fabricate well-controlled triangular nanoprism dimers and trimers and used a combination of transmission electron microscopy and electron energy loss spectroscopy to provide high resolution plasmon maps of these structures, which were then compared to electrodynamics simulations. ${ }^{87,88}$

Asymmetric coupled nanostructures: While not as common, there are also studies of plasmonic heterodimer coupling, showing new physical effects not observed in homodimers. For example, Halas and co-workers prepared size-mismatched Au NS-NS 

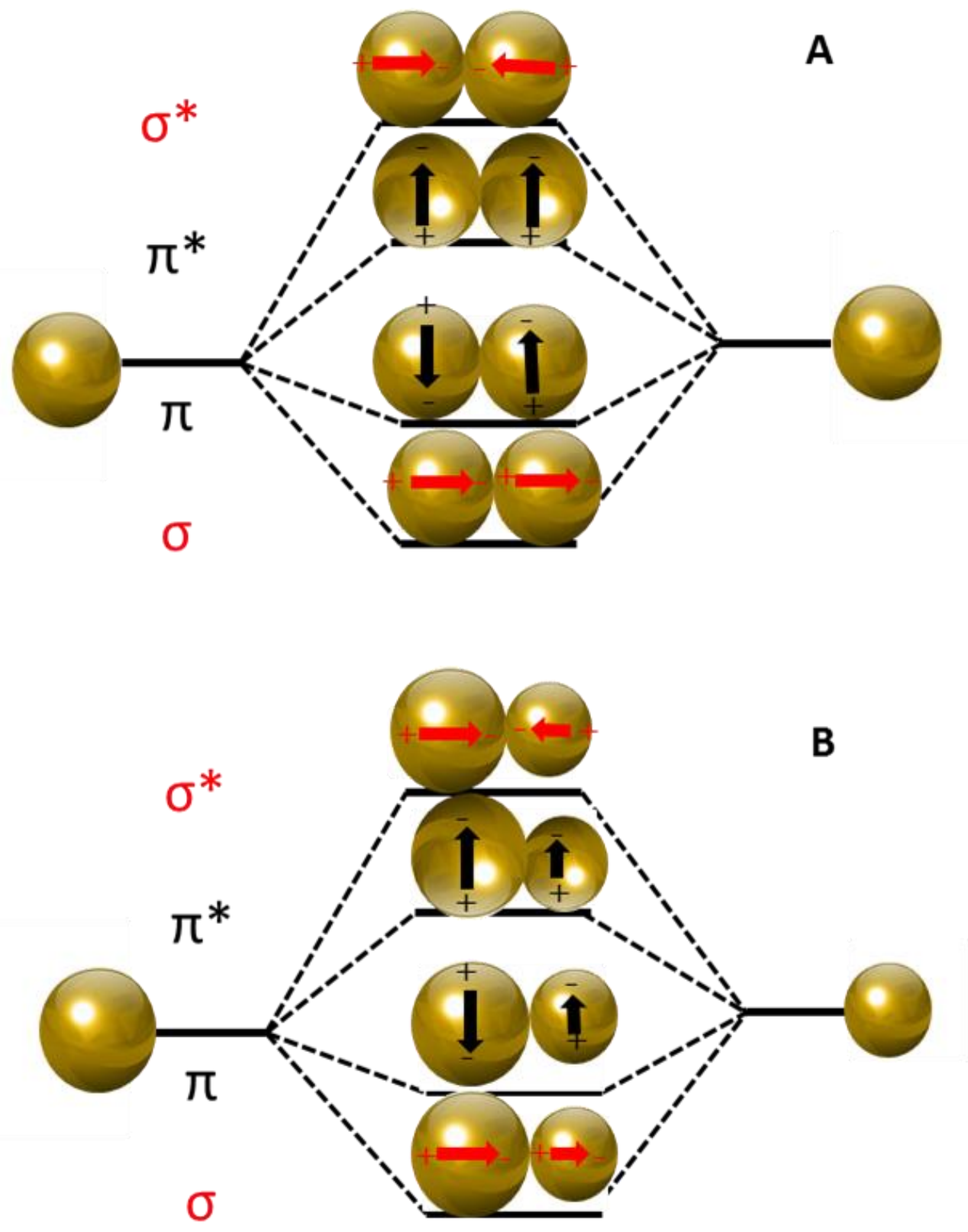

Figure 1.2 Schematic illustration of symmetric (A) and asymmetric (B) nanoparticle coupling. 
and shaped mismatched Au NS-Au nanoshell structures by an electromigration assembly method, observing avoided crossing behavior, Fano resonances, and asymmetric scattering properties. ${ }^{1} \quad$ Size-mismatched Ag NS-Ag NS and composition/sizemismatched Au NS-Ag NS structures have been synthesized to study the effect of symmetry breaking and allow observation of the out-of-phase plasmon mode in single dimers. ${ }^{78}$ Sannomiya et al. optically detected single binding events of small Au NSs attaching to larger $\mathrm{Au}$ NS through DNA hybridization. ${ }^{89}$ Pedro et al. examined the plasmonic hot spots of an $\mathrm{Ag}$ nanowire-Ag nanocube dimer configured face-to-face and face-to-edge and compared the enhancement factors for SERS applications. ${ }^{2}$ A dimer comprised of a small Ag nanocube on a much larger Au microplate has been synthesized as a promising SERS substrate..$^{90}$

\subsubsection{Larger Assemblies}

There are also several theoretical and experimental studies describing the optical properties of larger multi-nanoparticle homostructure and heterostructure aggregates. Mirkin and co-workers exploited the different optical properties of aggregated Au NSs compared to well-isolated $\mathrm{Au}$ NSs as a means to detect ss-DNA targets in solution colorimetrically. The strong coupling between aggregated Ag NSs leads to strong plasmon fields for single molecule SERS detection as another application of coupled aggregates. $^{72,73}$

More recently, there have been a number of studies describing the optical properties of core-satellite nanostructure aggregates. ${ }^{91,92}$ These are heterostructures involving one type of nanostructure as the core and several of another type of 
nanostructure surrounding the core as satellites. For example, shape-mismatched Au NR$\mathrm{Au}$ NS core-satellite structures were prepared by selectively binding the Au NSs completely around the $\mathrm{Au} \mathrm{NR}$, but also selectively to the ends or the sides. ${ }^{93}$ Coresatellite nanoassemblies with $50 \mathrm{~nm} \mathrm{Ag} \mathrm{NS}$ cores and $13 \mathrm{~nm}$ Au NS satellites linked by alkanedithiols have been prepared to understand plasmon coupling between compositionally heterogeneous nanoparticles, where the number of satellites varied and the distance ranged from 0.7 to $2.3 \mathrm{~nm} .{ }^{94}$ Quantitative analysis showed the LSPR band shifted $1.6 \pm 0.1 \mathrm{~nm} /$ satellite NS for the Au NS core, which is consistent with theoretical studies by Lee and coworkers. ${ }^{95}$ Using generalized multiparticle Mie (GMM) theory, they predicted an approximately $1 \mathrm{~nm}$ red-shit per satellite NS for a $100 \mathrm{~nm}-20 \mathrm{~nm} \mathrm{Au}$ NS-Au NS core-satellite assembly system.

\subsubsection{Simulation Methods}

A number of computational methods have been employed to simulate the electromagnetic wave propagation and scattering for nanoparticles including the finite element method (FEM), ${ }^{96}$ finite difference time domain method (FDTD), ${ }^{97}$ and the discrete dipole approximation (DDA). ${ }^{80}$ Each approach has advantages and disadvantages. In this thesis, only the DDA method has been used to simulate the LSPR spectra of single NP and NP/NS assemblies. The main advantage of the DDA method is that it is one of the most general methods, having a very broad range of applicability, limited only by available computational power. The other advantages include its relative conceptual simplicity, ease of use, and availability of public domain software. 
The DDA method is a general method to simulate scattering and absorption of electromagnetic waves by targets of arbitrary geometry. Initially the DDA was proposed by Purcell and Pennypacker ${ }^{98}$ and further developed by Draine and coworkers, who popularized the method by releasing a publicly available computer code DDSCAT. ${ }^{99}$ Simply stated, the DDA is an approximation of a larger object in terms of a finite array of discrete dipoles. The dipoles can interact with one another as well as the electric fields. A nanoparticle is represented by $\mathrm{N}$ polarizable dipoles arranged on a cubic grid. The grid spacing can restrict the size of the dipoles. Smaller grid spacing yields more dipoles and more structurally accurate representations of a nanoparticle while setting a higher requirement for the computational power.

\subsection{LSPR Sensing with Metallic Nanostructures}

The existence of the localized surface plasmon resonance (LSPR) is the ultimate reason for the interesting optical properties of metal NPs. For Au and Ag NPs, they exhibit a strong extinction band in the UV-visible and near IR region, which makes them highly desirable materials for various optical applications. ${ }^{56}$ The LSPR spectrum depends upon the shape, size, and composition of the nanoparticle, but also on the RI of the surrounding media and the interparticle distance of coupled nanoparticles. ${ }^{100,101}$ The latter two effects are exploited for sensing applications. The shift in the LSPR maximum wavelength ( $\left.\Delta \lambda_{\max }\right)$ of metal nanoparticles upon binding of analyte to the metal surface is determined by the following equation: ${ }^{54}$

$$
\Delta \lambda_{\max }=\left(\mathrm{S}_{\lambda} / \mathrm{V}_{\mathrm{s}}\right)\left[\Delta \mathrm{RI} * \mathrm{~N} * \mathrm{~V}_{\mathrm{A}}\right] \quad \text { Eq } 1.2
$$


Here, $\mathrm{S}_{\lambda}$ is the global RI sensitivity (in $\mathrm{nm} / \mathrm{RIU}$ or $\mathrm{eV} / \mathrm{RIU}$ ), $\mathrm{V}_{\mathrm{s}}$ is the sensing volume, $\Delta \mathrm{RI}$ is the change in the RI, N is the number of adsorbed analyte molecules, and $\mathrm{V}_{\mathrm{A}}$ is the volume of one molecule. As can be seen from equation 1.2, an increase in the RI of the binding molecule relative to the initial RI of the environment will result in a red shift in the LSPR band. A decrease will result in a blue shift. An increase in the analyte volume relative to the nanostructure sensing volume will increase the magnitude of the shift. For sensing applications, the magnitude of the shift in $\lambda_{\max }$ is monitored to quantitate the analyte. In order to improve the LSPR sensitivity, we can increase the number of molecules in the sensing volume, or increase the RI difference between the attached molecules from the initial environment.

Besides the size, shape, and composition of nanoparticles, the initial $\lambda_{\max }$ also has

a great effect on the magnitude of the shift in the LSPR peak. ${ }^{102,103}$ As the initial $\lambda_{\max }$ increases, the width of the LSPR band might also increase. The figure of merit (FOM), instead of relative shift, serves as a standard measure for assessing a nanoparticle's sensing potential because FOM takes the spectral line width into consideration. FOM is defined as follows: ${ }^{104}$

$$
\mathrm{FOM}=\mathrm{m}\left(\mathrm{eV} \mathrm{RIU}{ }^{-1}\right) / \text { fwhm }(\mathrm{eV}) \quad \mathrm{Eq} 1.3
$$

Here, $\mathrm{m}$ is the linear regression slope for the refractive index dependence and fwhm is the full width of the plasmon band at half maximum. Some nanoparticles exhibiting very large LSPR shifts have very broad resonance, which would lower the FOM. A narrow band is desirable since for a given shift in the peak position a larger change in signal will be detected comparing to a broad band. 


\subsubsection{Bulk RI Sensing}

LSPR sensing applications of nanoparticles are based on the fact that the changes of dielectric constant of surrounding media of nanoparticles results in measurable shifts in their LSPR peak position. Bulk RI sensing is to measure the shifts caused by the change of the bulk medium surrounding the nanoparticle. For local RI sensing, the shifts caused by molecules binding on the metal nanoparticle surface are monitored.

Ensemble particles: In solution based sensing experiments, nanoparticles are first dispersed in a solution with a known RI value. The spectra of the nanoparticles in solution are collected by a UV-vis spectrometer in transmission mode. The LSPR peak position is monitored in solutions with different RI. Pal and coworkers studied the effects of solvent and ligands on their LSPR peak positions of Au nanoparticles dispersed in solution. ${ }^{105}$ They found that $\lambda_{\max }$ of the Au nanoparticles has a strong dependence on the value of the RI and chemical nature of the surrounding solvent. When the solvent has no specific interaction with the surface of the Au nanoparticles, there is a linear relationship between the LSPR peak shift and the RI of the solvent. They also found that the $\lambda_{\max }$ of the LSPR shifted $\sim 3 \mathrm{~nm}$ for every one carbon atom in the carbon chain of alcohols surrounding the nanoparticles. Nanoparticles with different geometric shapes show different LSPR sensitivity in solution. There was a systemic study of the shape effect of Au nanoparticles on their RI sensing by Chen et al. ${ }^{106}$ In their study, Au nanoparticles of different shapes including NS, nanocubes, nanobranches, NRs, and nanobipyramids, were dispersed into water-glycerol solutions, whose RI was adjusted by changing the volume ratio of water and glycerol. For each shape, a plot of LSPR peak position as a function of RI of the surrounding medium revealed that the RI sensitivities were greatly dependent 
on the shape of the Au nanoparticles. The LSPR sensitivities generally increase as the $\mathrm{Au}$ nanoparticles become more asymmetric and their edges become sharper. Their experimental results show that spherical nanoparticles have a smallest RI sensitivity of 44 nm/RIU, while branched nanoparticles possessed the largest LSPR sensitivity of 703 $\mathrm{nm} / \mathrm{RIU}$.

Lithographically-fabricated nanostructures have been widely used in LSPR sensing experiments such as arrays of nanodiscs, nanoprisms, and nanorings. Ordered arrays of Au nanodiscs have been fabricated on substrates by colloidal lithography to study their optical properties. ${ }^{107}$ The LSPR sensitivity of the nanodisk arrays was investigated by varying the RI of the surrounding solvent. The experimental results demonstrated that the optical properties and sensing capabilities of nanodiscs can be easily tuned by changing their shape and size. NSL fabricated Ag nanoprism arrays were employed in exploration of their LSPR sensitivity to RI change of their surrounding medium. ${ }^{108} \mathrm{~A}$ Au nanoring is another nanostructure fabricated directly on substrates by colloidal lithography and was used in chemical and biosensing. ${ }^{109}$ The nanoring structures showed excellent capability in both bulk RI sensing and local sensing.

Single particles: Along with remarkable progress of nanoplasmonics over the past 10 years, single plasmonic nanoparticle sensors have introduced a completely new dimension to the sensing scale. The extension of LSPR sensing techniques to the single nanoparticle limit provides several important improvements that will enable new nanoscale sensing experiments. First, the absolute detection limit is dramatically reduced due to the small scale signal transducer. The sensitivity of single nanoparticle LSPR spectroscopy shows possibility of detecting single molecules, which is much lower than the instrumental resolution of typical UV-Vis spectroscopy. Another advantage of single 
nanoparticle sensing lies in the tiny nature without invasion to the surrounding system. It is an ideal platform for in vivo quantification of chemical species and monitoring of dynamic processes inside biological cells. The third advantage of single nanoparticle sensors is the high signal-to-noise ratio of the LSPR spectrum of individual nanoparticles. The scattering spectra of single nanoparticles have narrow line width compared to the extinction spectra of ensemble nanoparticles. Optical studies of single metal particles can date back to last century. Only until the recent 10 years, dark-field microscopy has been extensively involved in the study of individual metal nanoparticles. Rayleigh scattering spectra from single particles can be easily measured by coupling a dark-field microscope to a spectrometer and a CCD camera. Schultz and Feldmann reported their spectroscopic studies of single metal particles for the first time in $2000 .{ }^{110,111}$

Dark-field microscopy, as opposed to bright-field microscopy, employs an illumination technique used to enhance the contrast in unstained samples. It works by illuminating the sample with light that will not be collected by the objective lens, and thus will not form part of the image. This produces the classic appearance of a dark, almost black, background with bright objects on it. In this technique, white light from a halogen lamp is sent through a high numerical aperture (NA) dark-field condenser to illuminate the sample. In the center of this dark-field condenser, there is a circular opaque piece blocking some light from the light source, leaving a hollow cone of light directly transmitted and focused at the sample. Most of the light is directly transmitted, while some is scattered from the sample. The directly transmitted light simply misses the objective lens and is not collected. The scattered light from the sample (e.g. nanoparticles) is collected through a variable NA 100X oil immersion objective. The NA of the objective is set to 0.5 which is lower than NA of the dark-field condenser (0.95). 
The scattered light is then sent to a spectrometer and charge coupled device (CCD) camera and detector (Figure 1.3). Because the light transmitted through the condenser is not collected by the objective, only the light scattered by the nanoparticle is viewed, producing a dark-field image of the nanoparticles as a black background with some bright spots as shown in Figure 1.4, which is a Rayleigh scattering dark-field image of some Au NPs on a glass substrate with 100X oil objective lens. Nanoparticles with a different size and geometry scatter light at different wavelengths, which results in the different color dots in the image.

The sensitivity of single nanoparticle sensors usually is tested by immersing the nanoparticles into solvents with different RIs. The LSPR sensitivity is recorded as a wavelength shift in $\mathrm{nm}$ per RI unit (nm/RIU). The first report on investigation of plasmon resonance peak shifts due to RI changes of the surrounding solvents (oil) was described by Schultz and coworkers. ${ }^{64}$ In their study, they first collected scattering spectra for many individual Ag nanoparticles and then measured the spectral changes corresponding to the index of covering index oil with RI value from 1.44 to 1.56 . They determined that the average red shift for blue particles (usually spheres) was $1.6 \mathrm{~nm}$ per a change of 0.01 in index which is $160 \mathrm{~nm} /$ RIU unit. This is a very good demonstration of high sensitivity of single nanoparticle sensing. Later, the work from the Van Duyne group showed similar sensitivity of single spherical Ag nanoparticles, which is $161 \mathrm{~nm} /$ RIU. $^{112}$ Instead of immersing Ag nanoparticles in index oil, Van Duyne and coworkers placed their samples in a flow cell and covered it with organic solvents of different RI. Their research also showed that the shape of the nanoparticle can affect the RI sensitivity. More recently, Murray and coworkers published their experiment results on the study of LSPR sensitivity to bulk and local changes in the environment. ${ }^{113} \mathrm{Au}$ 

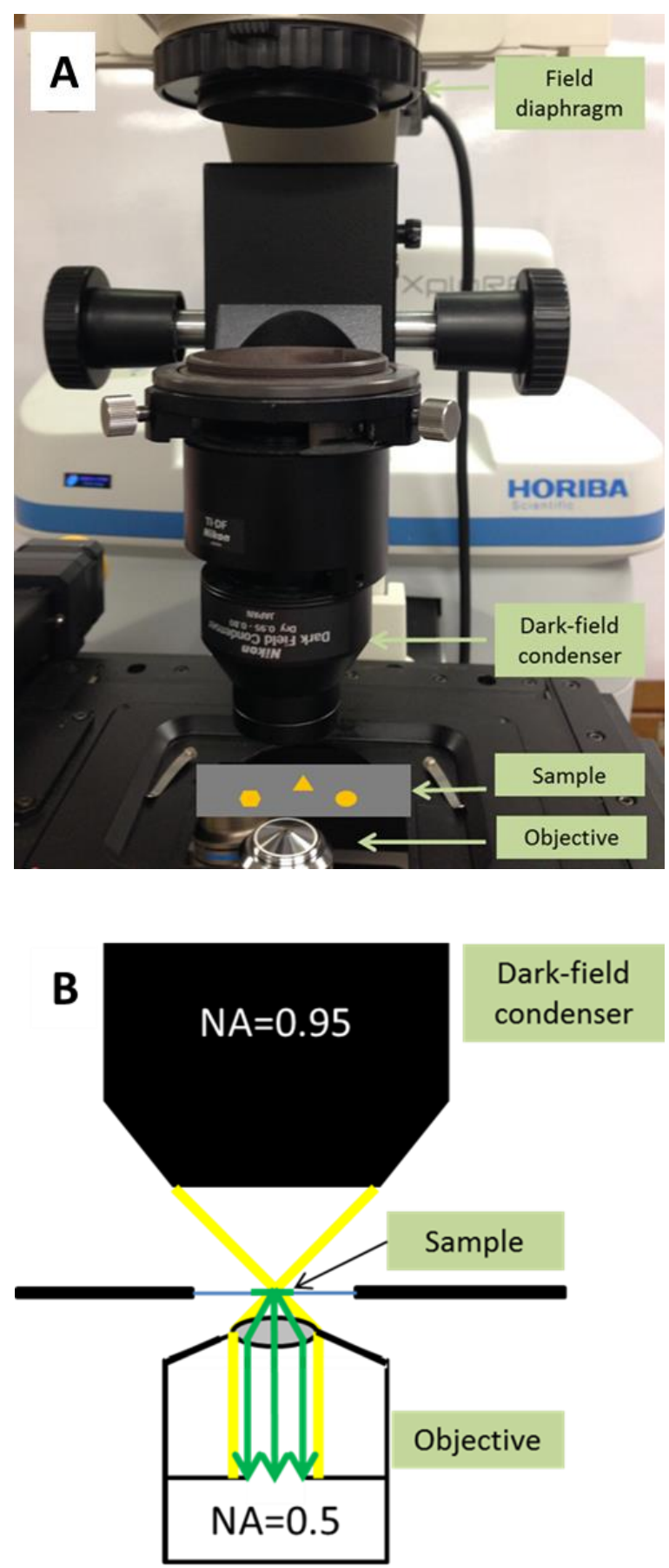

Figure 1.3 A) Apparatus for measuring dark-field scattering. B) Diagram showing the conical illumination. Yellow lines represent transmitted light missing the objective and green lines represent the scattered light detected by the objective. 


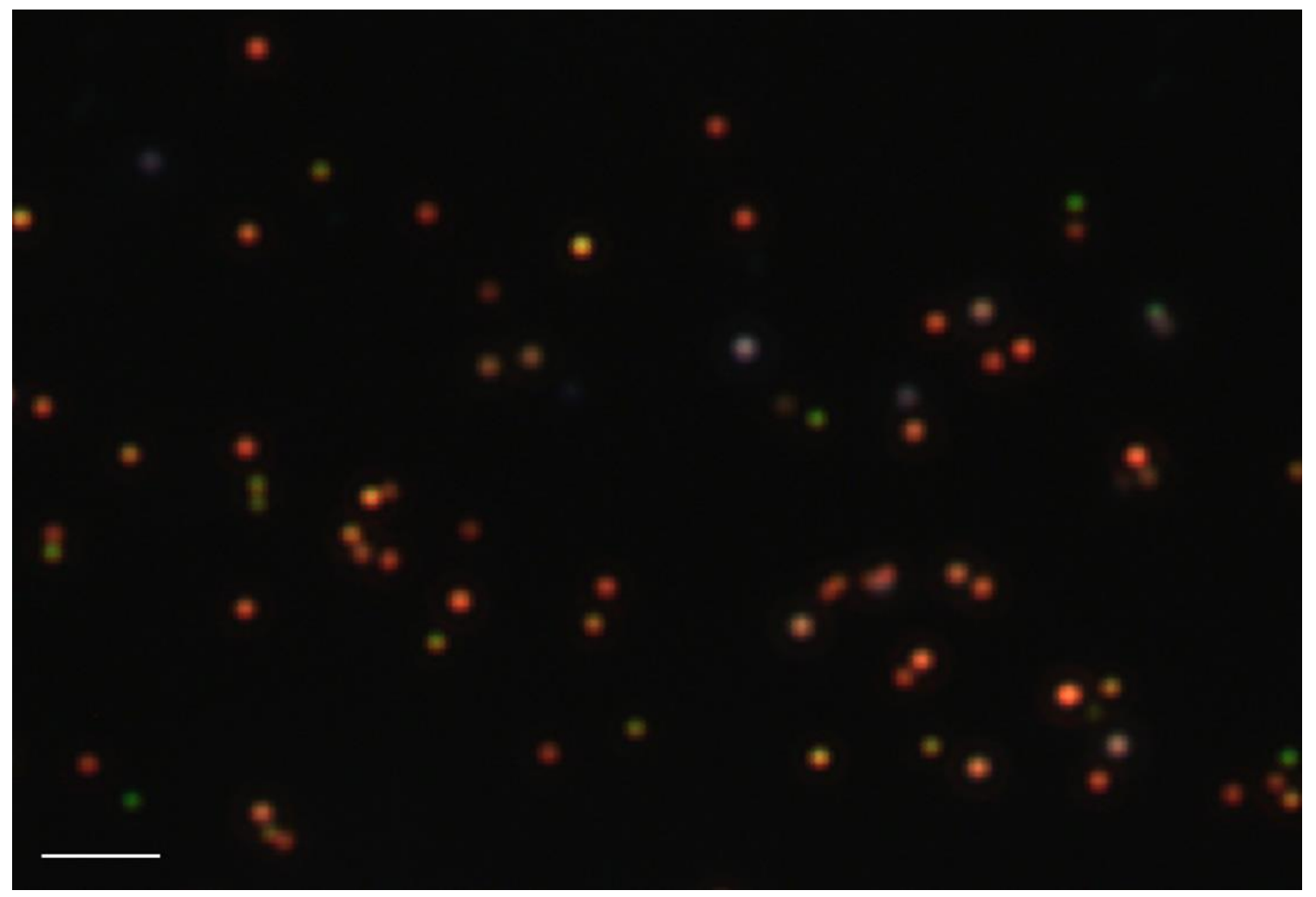

Figure 1.4 Dark-field microscopy images of $\mathrm{Au}$ nanoparticles on a glass slide at 100x magnification. Scale bar is $10 \mu \mathrm{m}$. 
nanoparticles were exposed to both nitrogen gas and methanol solvent and the corresponding LSPR band position measured to study RI sensing.

\subsubsection{Local Detection of Molecules}

Ensemble particles: Ghosh et al. studied the ligand binding effects on the LSPR of gold colloids. ${ }^{105}$ In their study, they explored the response of LSPR with the changes in RI induced by the ligand binding events to the Cetylpyridinium chloride-stabilized gold nanoparticles. They found that functionalized gold nanoparticles could detect changes in RI induced by analyte binding events. Higher aspect ratio nanostructures like NRs were used to perform local sensing measurements in solution. ${ }^{114}$ It has been reported that small changes in the aspect ratio of a Au NR leads to drastic changes in their optical properties. Au NRs with different aspect ratios were functionalized with different receptors and mixed up in a solution. The LSPR spectra of this colloidal solution exhibited three pronounced dipole peaks, corresponding to NRs with three different aspect ratios. Upon the complementary bonding of target molecules to different receptors on the NRs with different aspect ratio, a red shift in the corresponding LSPR peak occurred. This was the first application of Au NRs in multiplex biosensing.

Besides nanoparticles in solution, nanoparticles immobilized on substrates are also good biosensors for monitoring analyte binding events. For example, the wellknown model biotin-streptavidin has been used to show the LSPR sensitivity of gold nanoparticles on glass substrates. ${ }^{35}$ In these experiments, biotin was attached to Au NSs previously immobilized on glass through organic linkers. A red shift in the plasmon band was observed upon the binding of streptavidin molecules in the UV-Vis spectrum. With 
the optimized Au NS size, a detection limit of $0.83 \mathrm{nM}$ streptavidin was achieved. $\mathrm{Au}$ and Ag NSs immobilized on glass substrates have also been used to monitor antigenantibody recognition events. Antibodies (e.g. Human serum albumin) were attached to the metal nanoparticles, and the antigen (e.g. anti-human serum albumin) recognition was monitored via the red shift of light absorption when this binding event occurs. ${ }^{115}$ In our group, NPs grown on glass were applied in LSPR sensing of anti-immunoglobulin G. ${ }^{21}$ It has been reported in this study that the binding location of proteins on NPs has a great effect on the LSPR sensing. The sharp region like edges of the NP exhibits higher sensitivity than flat terrace areas. Apart from colloidal nanoparticles, a number of biosensing applications of Ag nanoparticles fabricated by NSL methods have been reported. Ag nanoprism arrays are also very useful in monitoring binding events at the surface of $\mathrm{Ag}$ surfaces. ${ }^{116}$ Yonzon and coworkers studied the association and dissociation constants of concanavalin A molecules binding to monosaccharides absorbed on Ag nanoprism arrays. More recently, it has been reported that Ag nanoprism arrays were applied to detect biomarkers for Alzheimer's disease in biological samples. ${ }^{7}$

Single particles: Most studies about single particle sensing focus on application as biosensors since the single particle sensor could be used as a tiny probe to collect local biological information in biological tissues or in living cells on a nanometer scale. Typical biosensing utilizes the resonance shift due to the local RI change upon bimolecular adsorption. The current research of single particle biosensing has already shown a wide variety of applications that mostly take advantage of their uniquely small sensing volume and non-invasive nature to biological systems. The sensing scale is now reaching countable numbers of molecules. ${ }^{117-119}$ 
Molecular adsorption biosensors were developed based on molecular adsorption on the nanoparticle surface and resulting LSPR shifts. Real-time biomolecular adsorption on a single particle was first shown in 2003 by Raschke et al. In their work, Au nanoparticles were functionalized with biotinylated bovine serum albumin to selectively detect streptavidin with a detection limit of a few hundred streptavidin molecules. Compared to a spherical nanoparticle, single NR sensors have shown larger resonance shifts upon molecular adsorption. ${ }^{120}$ Using single NRs with optimal aspect ratios, a sensitivity of $1 \mathrm{nM}$ has been achieved for streptavidin detection. Individual NRs have also been used to locally monitor protein adsorption on biological membranes of lipid bilayers. ${ }^{121}$ Minimizing the detection number of analytes becomes a true advantage over ensemble measurements.

The ultimate goal of biosensing is to detect a single binding or unbinding event. To achieve this goal, there are still many difficulties since the increase in RI by a single molecule binding is very small. Many efforts have been made to increase the signal-tonoise ratio by using sharp edged particles with higher sensitivity such as nanoprisms or nanostars, or high RI analytes with some labels. The detection of single binding events by monitoring the LSPR peak shift has been reported by Sanomiya et al. using labeled nanoparticles. ${ }^{89}$ The strategy was to immobilize $100 \mathrm{~nm}$ gold particles on a substrate and then functionalize them with cDNA strands. Stepwise LSPR peak shifts have been observed when DNA molecules labeled with $20 \mathrm{~nm}$ gold nanoparticles bind to cDNA at the single event level. To date, no label-free single molecule adsorption process has been experimentally observed with resonance-shift based sensing. Only theoretical calculations show that it is possible to push the resonance shift caused by singe molecule adsorption to a measureable range using particles with sharp edges or assembled 
particles. $^{122,123}$ Instead of adsorption dynamics of single molecules, single molecule desorption of antibodies from the tips of plasmonic bipyramids has been achieved. ${ }^{124}$

\subsection{Summary and Accomplishments}

Chapter III contains results and discussion on the effect of NS location on the LSPR shift when attaching to a NP. Chapter IV shows the effect of NS size on the LSPR shift when coupling with a NP. Chapter V investigates the effect of the organic ligand used to link the Au NS to a NP on the LSPR spectra pattern and shift. Chapter VI compares the sensitivity of different shaped NPs, including triangular, hexagonal, and circular NPs and RI sensing using LSPR spectroscopy. Chapter VII provides a summary of this research and future directions. With the development of a strategy to correlate the dark-field light scattering spectra of individual nanostructures with SEM and AFM images of the same nanostructures, we were able to investigate several interesting optical properties of coupled NP/NS structured as well as the factors that affect the coupling, including attachment location of the NS on the NP, the size of the NS, and the organic ligands linking the NS and NP. The NP/NS coupling is important in amplifying LSPR sensing signals and improving SERS sensitivity. We studied location dependent properties of NP/NS coupling, which could be used for spatial 3D sensing. The effect of NS size has been investigated and is important for LSPR sensing amplification and molecular ruler applications. The effect of linkers is also important in both fundamental studies of coupled nanostructures and their applications. RI sensing with different NPs provides useful information for LSPR sensing. This research provides fundamental 
information about the optical properties of asymmetrically coupled NP/NS assemblies and useful information for designing 3D plasmonic sensors. 


\section{CHAPTER II}

\section{EXPERIMENTAL}

\subsection{Chemicals and Solutions}

Citric acid trisodium salt, L-Ascorbic acid (99\%), sodium borohydride (98.5\%), cetyltrimethylammonium bromide (CTAB, 95\%), mercaptopropyltrimethoxysilane (MPTMS, 95\%), 1-hexanethiol (1-HT, 95\%), 4-aminothiophenol (4-ATP, 97\%), Cystamine dihydrochloride (96\%), Hydrogen peroxide solution (30 wt. \% in $\mathrm{H}_{2} \mathrm{O}$ ), isopropanol (IPA, 99.9\%), ethyl alcohol (ACS/USP grade), and acetone (ACS/USP grade) were purchased from Sigma-Aldrich. $\mathrm{HAuCl}_{4} * 3 \mathrm{H}_{2} \mathrm{O}$ was synthesized from metallic $\mathrm{Au}$. $0.01 \mathrm{M}$ of $\mathrm{HAuCl}_{4}$ solution was prepared by dissolving solid $\mathrm{HAuCl}_{4} * 3 \mathrm{H}_{2} \mathrm{O}$ into nanopure water (Barnstead, resistance $\approx 18 \mathrm{M} \Omega . \mathrm{cm}$ ) and used for the synthesis of the $\mathrm{Au}$ nanospheres (NSs) and nanoplates (NPs). All other aqueous solutions were also prepared with nanopure water.

Aqua Regia: Aqua regia solution is a highly corrosive mixture of concentrated nitric acid $\left(\mathrm{HNO}_{3}\right)$ and hydrochloric acid $(\mathrm{HCl})$, with a volume ratio of $\mathrm{HNO}_{3}: \mathrm{HCl}=1: 3$. It is used for cleaning Au from the glassware and stir bars. The glassware and stir bars were put in fresh aqua regia solution for 30 minutes, and then rinsed thoroughly with deionized water and nanopure water. 


\subsection{Substrates}

Silicon: Air-oxidized silicon substrates $\left(\mathrm{Si} / \mathrm{SiO}_{\mathrm{x}}\right)$ were purchased from Silicon Quest International (San Jose, $\mathrm{CA}$ ). $\mathrm{Si} / \mathrm{SiO}_{\mathrm{x}}$ was cut with a diamond pen and cleaned in acetone $(20 \mathrm{~min})$, ethanol (20 $\mathrm{min})$, and IPA (20 $\mathrm{min})$ by ultra-sonication, and dried under $\mathrm{N}_{2}$. These substrates were used for growing metal nanostructures directly on them in this research.

Glass: Glass slides were purchased from VWR. Glass substrates with etched number markings were prepared using photolithography followed by a buffered oxide etch in the University of Louisville Micro Nano Technology Center. Glass slides were used to grow the metal nanostructures directly on the surface for single particle dark-field scattering studies. Glass slides were cleaned using the same procedure as silicon.

\subsection{Synthesis of 3-5 nm Diameter Gold Nanosphere (NS) Seeds.}

Small 3-5 nm diameter Au NSs, termed "Au seeds" were synthesized by adding $0.6 \mathrm{~mL}$ of ice cold $0.1 \mathrm{M} \mathrm{NaBH}_{4}$ to $19 \mathrm{~mL}$ of an aqueous solution of $2.5 \times 10^{-4} \mathrm{M}$ trisodium citrate and $2.5 \times 10^{-4} \mathrm{M} \mathrm{HAuCl}_{4} * 3 \mathrm{H}_{2} \mathrm{O}$ under stirring. The Au seed solution was used to synthesize Au NPs $2 \mathrm{~h}$ after their preparation as described in section 2.6.

\subsection{Preparation of 25 nm Diameter Citrate-Stabilized Au NSs.}

$25 \mathrm{~nm}$ diameter citrate-stabilized Au NSs were synthesized by adding $10 \mathrm{~mL}$ of a $1 \%$ trisodium citrate solution to $100 \mathrm{~mL}$ of a boiling $2.5 \times 10^{-4} \mathrm{M} \mathrm{HAuCl}_{4} * 3 \mathrm{H}_{2} \mathrm{O}$ solution. The mixed solution was kept stirring and boiling for $1 \mathrm{~h}$ and cooled down to 
room temperature with stirring before use. The $25 \mathrm{~nm}$ diameter $\mathrm{Au}$ NS solutions were used $1 \mathrm{~h}$ after preparation.

\subsection{Preparation of $\sim 51 \mathrm{~nm}$ and $\sim$ 13nm Diameter Citrate-Stabilized Au NSs.}

Au NSs with sizes $\sim 51 \mathrm{~nm}$ and $\sim 13 \mathrm{~nm}$ were synthesized using the procedure described by Wang and coworkers to chemically synthesize quasispherical, citratestabilized $\mathrm{Au} \mathrm{NSs}$ via $\mathrm{H}_{2} \mathrm{O}_{2}$ reduction of $\mathrm{HAuCl}_{4}{ }^{125}$ Briefly, $100 \mu \mathrm{L}$ or $20 \mu \mathrm{L}$ of 1 wt $\%$ $\mathrm{HAuCl}_{4} \cdot 3 \mathrm{H}_{2} \mathrm{O}$ was added to an aqueous solution containing $4.90 \mathrm{~mL}$ of nanopure water, respectively. Following the addition of $\mathrm{HAuCl}_{4} \cdot 3 \mathrm{H}_{2} \mathrm{O}, 25 \mu \mathrm{L}$ of 1 wt $\%$ citrate was added into the solution under stirring. Then $5 \mathrm{~mL}$ of $30 \mathrm{wt} \% \mathrm{H}_{2} \mathrm{O}_{2}$ was injected and followed by the immediate addition of $10 \mu \mathrm{L}$ or $20 \mu \mathrm{L}$ of $4 \mathrm{~nm}$ diameter Au seeds while stirring for $51 \mathrm{~nm}$ or $13 \mathrm{~nm}$ NSs synthesis.

\subsection{Substrate Functionalization.}

After cutting and cleaning by 3 different solvents, glass and $\mathrm{Si} / \mathrm{SiO}_{\mathrm{x}}$ slides were functionalized with 3-mercaptopropyltrimethoxysilane (MPTMS) by soaking in a solution of $10 \mathrm{~mL}$ of IPA, $100 \mu \mathrm{L}$ of MPTMS, and 4 drops of water at a temperature of $95{ }^{\circ} \mathrm{C}$ for 30 minutes. After cooling down to room temperature, samples were rinsed thoroughly with IPA and dried under $\mathrm{N}_{2}$.

\subsection{Synthesis of Au NPs Directly on Glass Surfaces with Optimal Size and Coverage.}

$\mathrm{Au}$ nanostructures were synthesized directly on the glass slides using a seedmediated growth procedure reported by our group recently as shown in the Scheme in 
Figure $2.1,{ }^{21}$ but with a small modification designed to decrease the size and density of the NPs on the surface so that they would be amenable to single nanoparticle dark-field scattering studies. The glass slides were first functionalized with 3mercaptopropyltrimethoxysilane (MPTMS) as described in 2.5. After rinsing thoroughly with 2-propanol and drying under $\mathrm{N}_{2}$, the MPTMS-functionalized slides were then placed into a 1:10 diluted solution of $\mathrm{Au}$ seeds for $5 \mathrm{~min}$, rinsed with Nanopure ${ }^{\circledR}$ water, and dried under $\mathrm{N}_{2}$. Next, the samples were placed into a Au growth solution containing 9.0 $\mathrm{mL}$ of $0.1 \mathrm{M}$ cetyltrimethylammonium bromide (CTAB), $450 \mu \mathrm{L}$ of $0.01 \mathrm{M}$ $\mathrm{HAuCl}_{4} * 3 \mathrm{H}_{2} \mathrm{O}$, and $50 \mu \mathrm{L}$ of $0.1 \mathrm{M}$ ascorbic acid for $5 \mathrm{~min}$, followed by rinsing with Nanopure ${ }^{\circledR}$ water, and drying under $\mathrm{N}_{2}$. In our previous work, we placed them in a growth solution for $1 \mathrm{~h}$, which led to much larger Au NPs that are unsuitable for our single-nanoparticle scattering studies because of their broader, more red-shifted and complex scattering peaks. Following growth, the nanostructures on the glass slides contain a mixture of Au NPs and Au NSs. The NSs and some NPs were removed by sonication for $10 \mathrm{~min}$. This procedure led to samples with $>90 \% \mathrm{Au}$ NPs at a low areadensity, which is necessary for the single nanoparticle dark-field scattering experiments. 

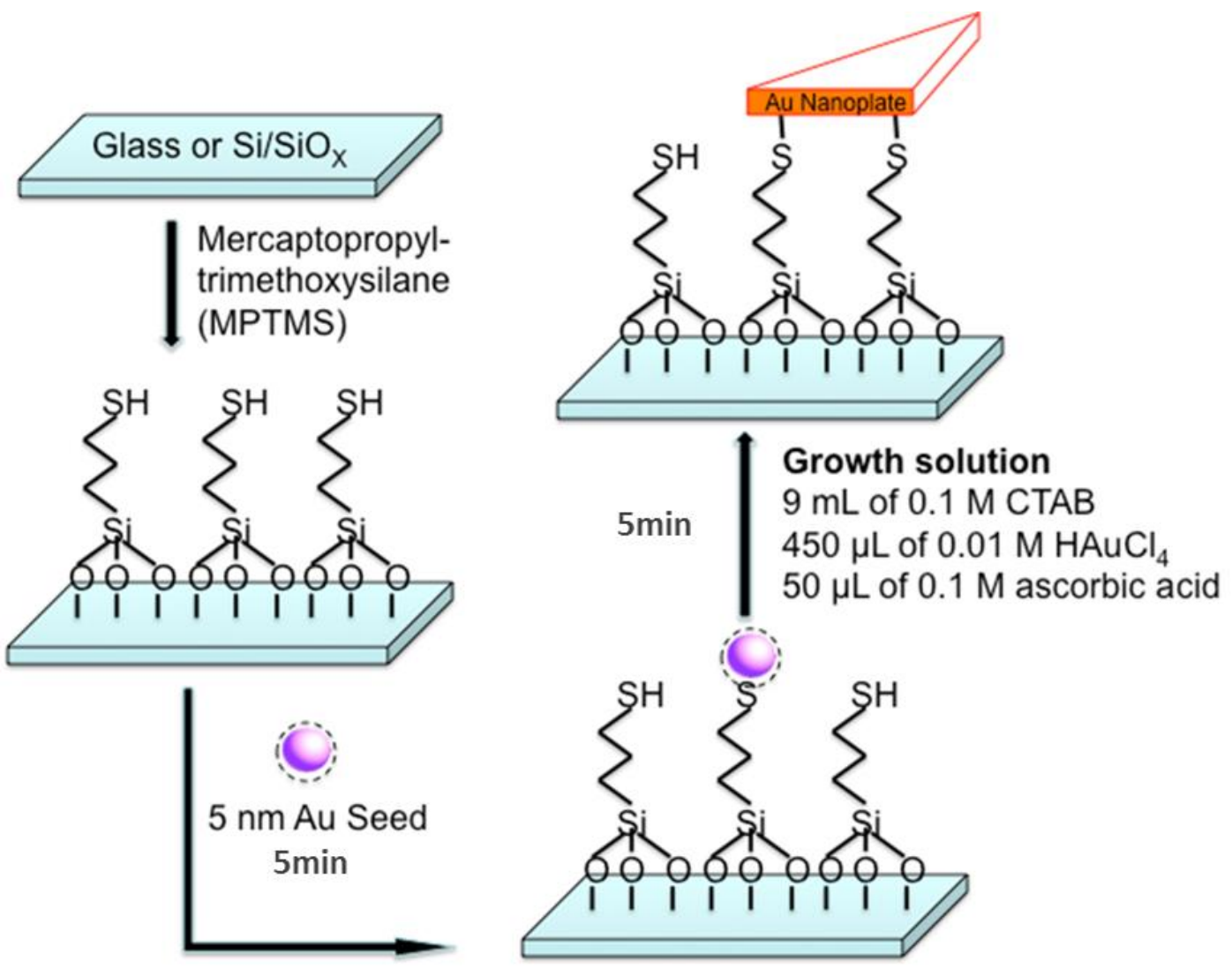

Figure 2.1 A schematic illustration of the growth of Au nanostructures directly on the surface of glass and $\mathrm{Si} / \mathrm{SiO}_{\mathrm{X}}$ substrates by the seed-mediated growth procedure. 


\subsubsection{Optimization of Au NP Coverage for Single Particle Dark-Field (DF) Scattering.}

Using the previous procedure reported by our group to grow Au NPs, the coverage of NPs is very high and there is not enough spatial resolution to collect scattering from a single NP. Two parameters have been investigated to get the optimal coverage of $\mathrm{Au}$ NPs, the concentration of $\mathrm{Au}$ seed solution and the soaking time in $\mathrm{Au}$ seed solution. The fresh made Au seeds solution have a concentration of $2.5 \times 10^{-4} \mathrm{M}$. The coverage should be reduced by diluting the seeds solution in a 1:2 and 1:10 ratio with nanopure water. Dark-field microscopy (DFM) and scanning electron microscopy (SEM) images were analyzed to get the Au NPs coverage with optimal spatial resolution. The results show that Au NP samples synthesized from 1:10 diluted Au seeds solution gives the optimal coverage. Based on the DFM images of Figure 2.2 A and C, we can see that the NPs were too crowded to resolve individual NPs without dilution of the Au seeds solution. After dilution, most of the NPs were well separated by DFM. Statistical calculations show that there are 25 NPs in a $15 \mu \mathrm{m} \times 15 \mu \mathrm{m}$ area for samples without dilution and 10 NPs with dilution which is 2.5 times less NPs. The synthesis with Au seeds diluted by 1:10 was adopted in the synthesis of all Au NP samples for all single particle scattering experiments in this research. 

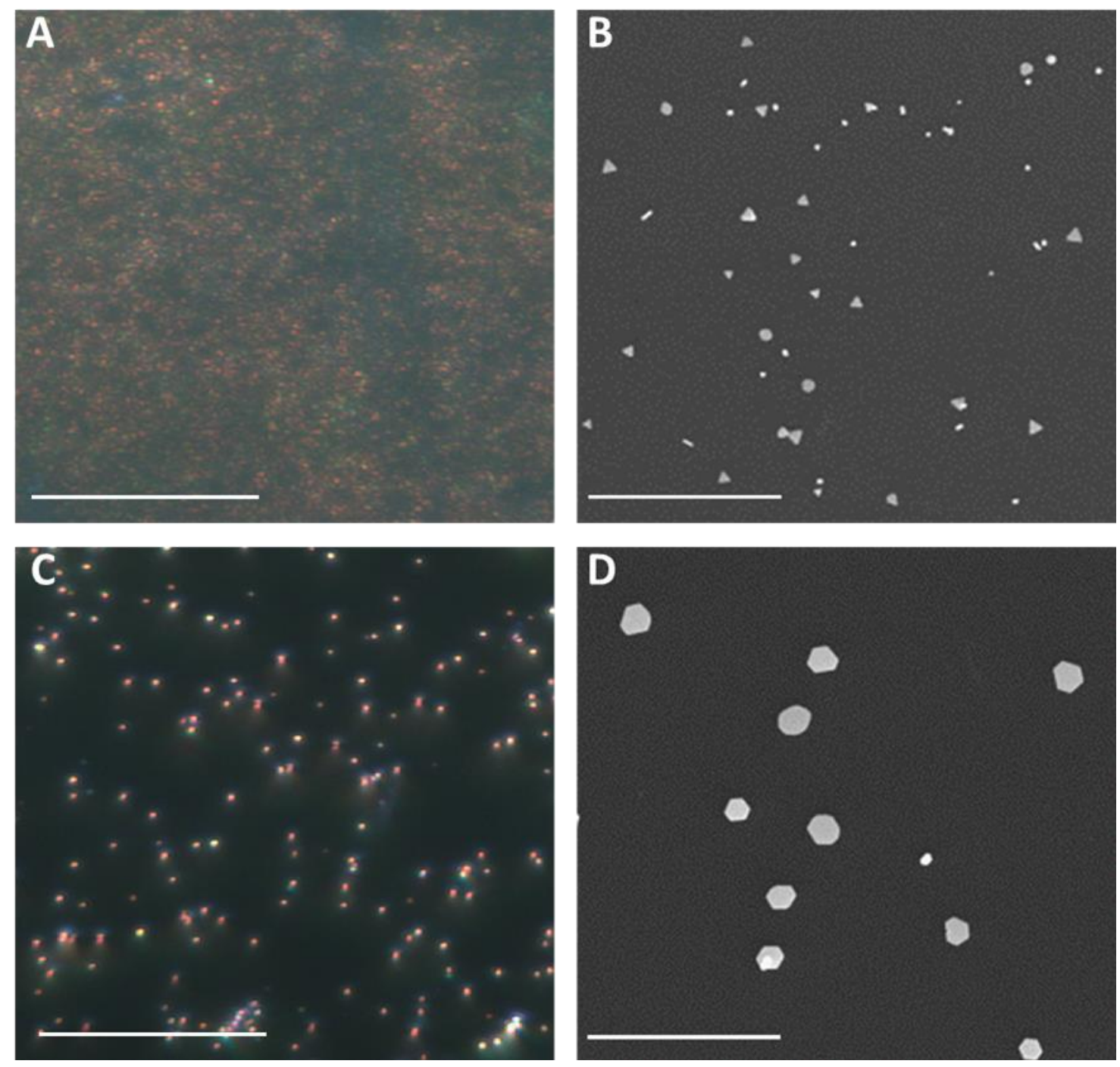

Figure 2.2 Dark-field and SEM images of Au NP samples synthesized from Au seeds solution without dilution (A and B) and with 1:10 dilution (C and D). Scale bars in DF images are $40 \mu \mathrm{m}$ and in SEM images are $5 \mu \mathrm{m}$. 


\subsubsection{Optimization of Au NP Size for Single Particle DF Scattering.}

After the optimization of $\mathrm{Au}$ NP coverage, there is enough spatial resolution for performing single NP scattering measurements. However, after dilution the Au NPs were mostly hexagonal and $\sim 500 \mathrm{~nm}$ wide (Figure 2.2D) as compared to being mostly triangular and $\sim 100 \mathrm{~nm}$ wide without dilution (Figure $2.2 \mathrm{~B}$ ). This is due to the larger ratio of $\mathrm{AuCl}_{4}^{-}$ions relative to the $\mathrm{Au}$ seeds on the surface which leads to larger grown NPs as expected. Triangular shaped Au NPs can convert to hexagonal shapes when grown larger. The scattering spectrum of a single nanoparticle will become broadened dramatically well above the size of $100 \mathrm{~nm} .{ }^{126}$ In order to control the size of NPs around $100 \mathrm{~nm}$ with low coverage still, the growth time of the samples was reduced from $60 \mathrm{~min}$ to $5 \mathrm{~min}$. As a result, the size of the Au NPs decreased from around $500 \mathrm{~nm}$ to $100 \mathrm{~nm}$ (Figure $2.3 \mathrm{~A}$ and C). NPs as large as $500 \mathrm{~nm}$ in width have a broad dipolar plasmom band and many other higher order modes not observed in other smaller NPs as shown in

Figure $2.3 \mathrm{~B}$ which agrees with observations in the literature. ${ }^{127}$ In contrast, NPs with widths around $100 \mathrm{~nm}$ have a much narrower dipolar peak without the appearance of high order modes. The optimized time in growth solution for the synthesis of NPs was therefore $5 \mathrm{~min}$. 

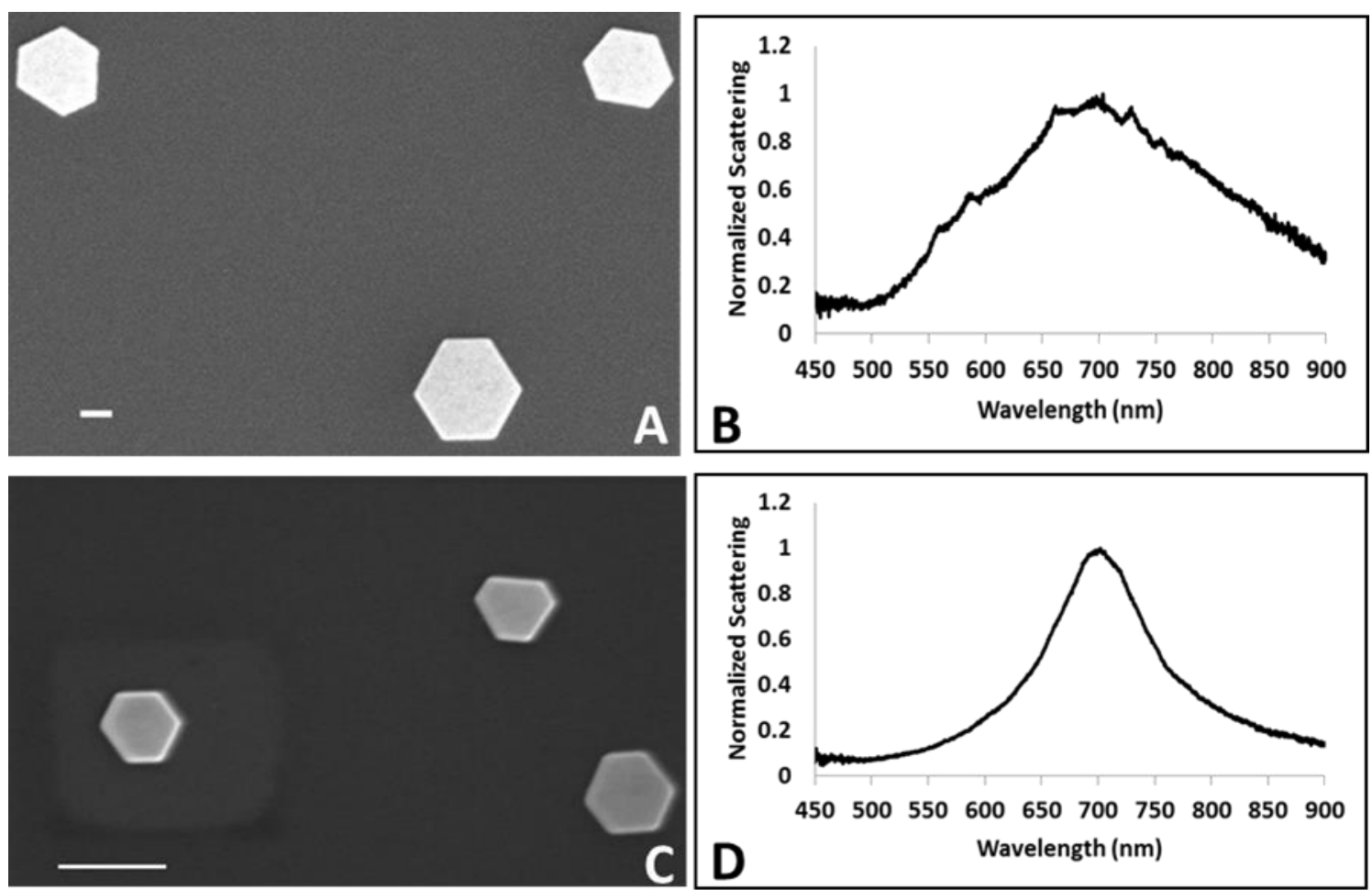

Figure 2.3 SEM images and scattering spectra of Au NPs samples synthesized in growth solution for $60 \mathrm{~min}(\mathrm{~A}$ and $\mathrm{B}$ ) and $5 \mathrm{~min}(\mathrm{C}$ and $\mathrm{D})$. Scale bars in the SEM images are $200 \mathrm{~nm}$. 


\subsection{Attachment of $25 \mathrm{~nm}$ Au NSs to Au NPs via Organic linkers.}

\subsubsection{The pH Value of 4-ATP Monolayer Protonation.}

One of the most popular strategies to assemble nanostructures is the utilization of bi-functional organic linkers such as dithiol and aminothiol, since both of these organic molecules have two terminal groups which can attach to the Au surface under certain experimental conditions. 4-ATP is a good bi-functional organic ligand that allows the coupling of citrate-coated Au NSs to Au NPs, since it has a thiol group that attaches well to the $\mathrm{Au}$ NPs and the $\mathrm{NH}_{2}$ group that, when protonated as $\mathrm{NH}_{3}{ }^{+}$, allows electrostatic attachment of negatively-charged, citrate-coated Au NSs. The $\mathrm{pH}$ value of the Au NS solution dictates the level of $\mathrm{NH}_{2}$ protonation on 4-ATP and has a large impact on NS attachment. The original prepared $25 \mathrm{~nm}$ Au NS solution has a $\mathrm{pH}$ value around 5.5. The surface coverage of Au NSs on a 4-ATP-modified Au planar electrode is only about 10 particles over a $500 \mathrm{~nm} \times 500 \mathrm{~nm}$ area by soaking the electrode in a Au NS solution at $\mathrm{pH}$ 5.5. When the $\mathrm{pH}$ decreased to 4.8 , the coverage increased dramatically from tens to hundreds which can be easily seen in the SEM image in Figure 2.4. There are around 300 NSs over a $500 \mathrm{~nm} \times 500 \mathrm{~nm}$ planar Au surface by soaking the planar Au in a Au NS solution at $\mathrm{pH} 4.8$. 

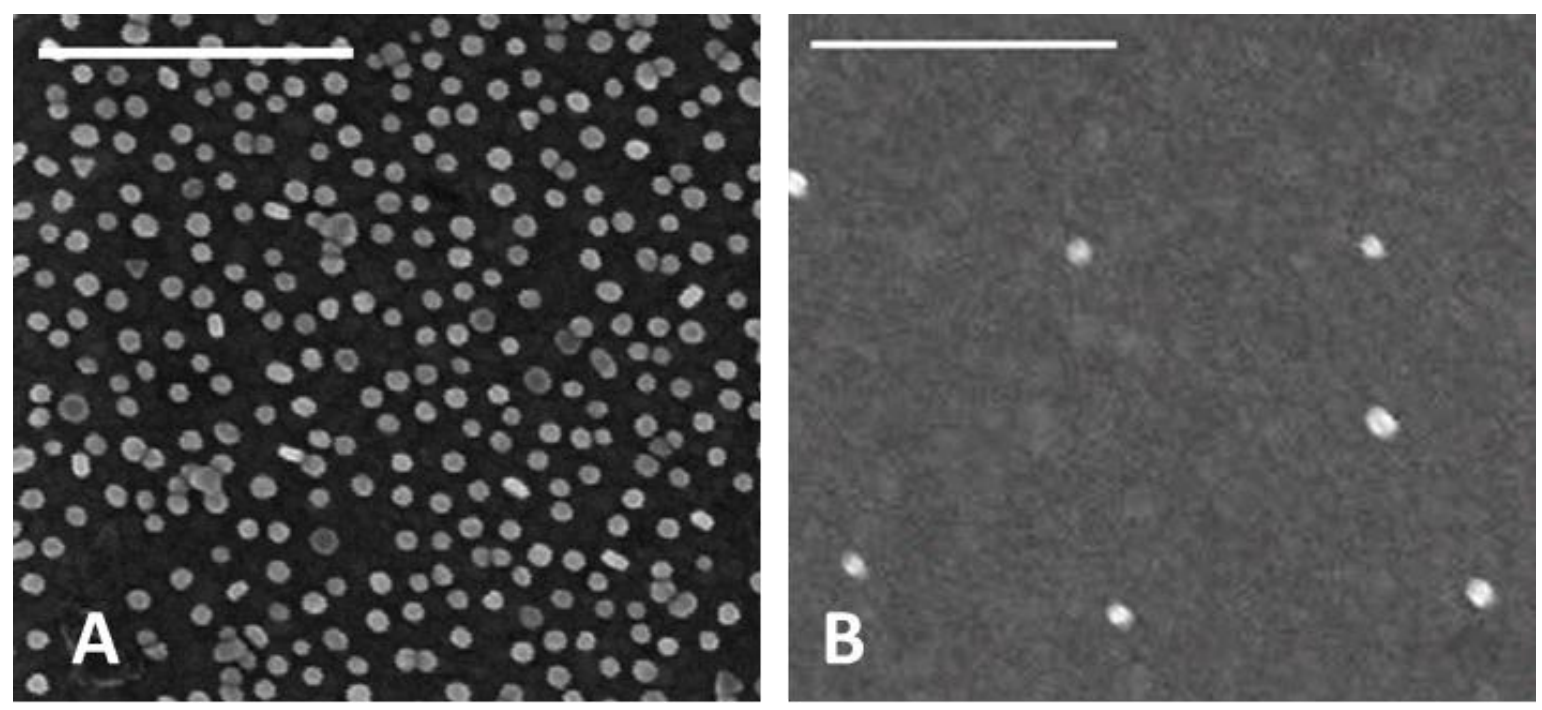

Figure 2.4 SEM images of NSs attached to a planar Au film surface by soaking in a Au NS solution at $\mathrm{pH} 4.5$ (A) and 5.5 (B). The scale bars in the SEM images are $200 \mathrm{~nm}$. 


\subsubsection{Ozone Cleaning of Au NP Surface}

When the substrate is changed from a planar $\mathrm{Au}$ film to freshly synthesized $\mathrm{Au}$ NPs, only the edge of plates show attachment of $25 \mathrm{~nm}$ diameter Au NSs after soaking in $5 \mathrm{mM}$ 4-ATP overnight and then soaking in the Au NS solution at $\mathrm{pH} 4.5$ for $30 \mathrm{~min}$ (SEM image in Figure 2.5 A). It seems there is something on the NP surface which hinders the attachment of Au NSs there. Au NPs were prepared by a seed-mediated growth method in which cetyltrimethylammonium bromide (CTAB) is widely used as a shape-directing surfactant. It has been reported that when using the same method to synthesize Au NRs, the CTAB surfactant selectively forms a densely packed dynamic layer around the sidewall of a growing Au NR with its two ends free from CTAB. The densely-packed CTAB layer obstructs thiol molecules from accessing the Au surface to bind to the $\mathrm{Au}$ through a strong covalent $\mathrm{Au}-\mathrm{S}$ linkage. For this reason, thiol molecules have been attached only onto the two CTAB-free ends. ${ }^{128}$ In a similar fashion, it may be that the 4-ATP attaches selectively to the edges of the Au NPs in the presence of CTAB and the Au NSs also selectively attach to the edge of the NPs since they bind to the 4ATP. If the surface is blocked by CTAB or other organic residues, exposure to ozone for a few minutes should remove these organic molecules. An experiment was performed to confirm this hypothesis. Freshly made Au NP samples were placed in ozone for $10 \mathrm{~min}$ after synthesis, then overnight in $5 \mathrm{mM}$ 4-ATP, and then immersed in a solution of $25 \mathrm{~nm}$ Au NSs at pH4.8 for 30 min. From the SEM image in Figure 2.5 B, it appears that ozone removes some surface blockage, likely from $\mathrm{CTAB}$, and allows uniform attachment of $\mathrm{Au}$ NSs over the Au NPs, including both edges and top terrace region. 

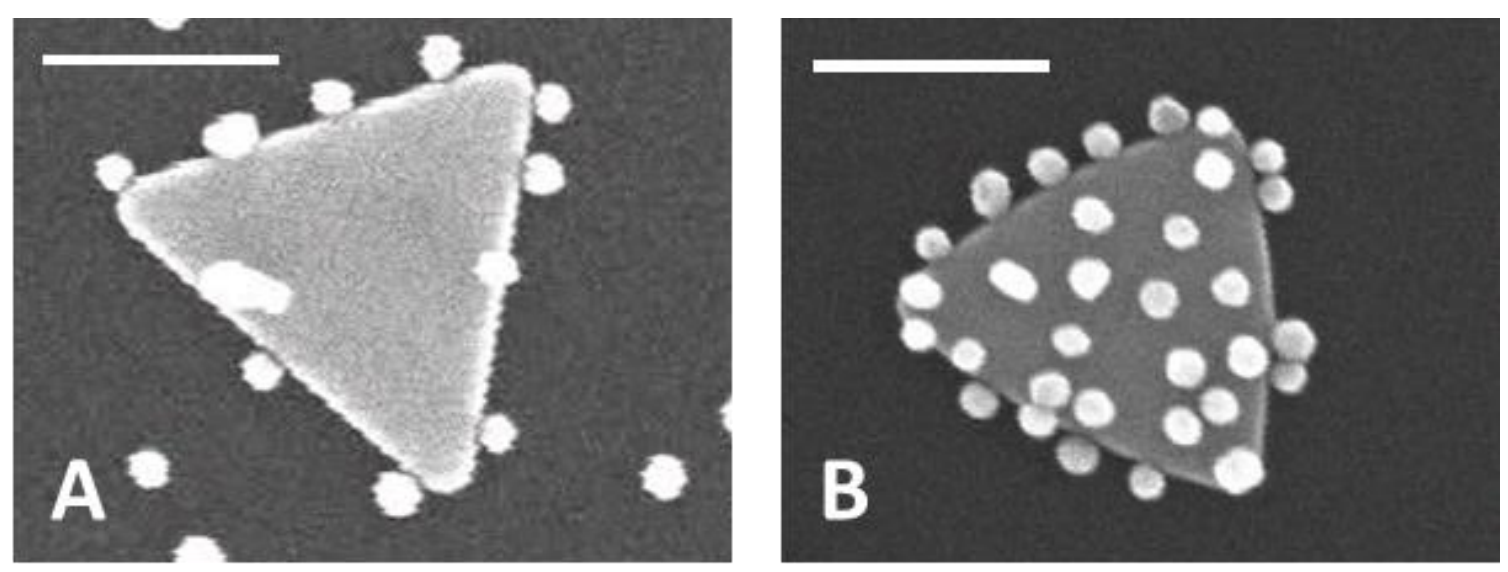

Figure 2.5 SEM images of NSs attached to 4-ATP-functionalized Au NPs without exposure to ozone (A) and after exposure to ozone for $10 \mathrm{~min}(\mathrm{~B})$. The scale bars in the SEM images are 100 nm. 


\subsection{Controlled Attachment of Au NSs to the Vertex/Edge Sites of Au NPs.}

Based on the work on planar Au films and Au NPs, we used ozone cleaning and a $\mathrm{Au}$ NS solution at $\mathrm{pH} 4.8$ for all experiments involving the coupling of $\mathrm{Au}$ NSs to $\mathrm{Au}$ NPs. To controllably attach the NSs only to the vertex or edge sites of the NPs, we used a ligand-place exchange method as described by our group previously for protein attachment to edge and vertex sites. ${ }^{21}$ We placed the glass/Au NPs sample in a $1 \mathrm{mM}$ ethanol solution of hexanethiol (HT) overnight. We rinsed thoroughly with ethanol, dried under $\mathrm{N}_{2}$, and then exchanged the HT monolayer with 4-ATP by placing the sample into a $6 \mathrm{mM}$ ethanol solution of 4-ATP for 1 hour. We again rinsed thoroughly with ethanol and dried under $\mathrm{N}_{2}$. The substrates were then immersed in a $10 \mathrm{~mL}$ solution of $\sim 25 \mathrm{~nm}$ diameter Au NSs for 30 min, rinsed thoroughly with nanopure water, and dried under $\mathrm{N}_{2}$. HT ligands were assembled onto the Au NPs and then place-exchanged with 4-ATP ligands. 4-ATP is a good bi-functional organic ligand that allows the coupling of citratecoated Au NSs to Au NPs, since it has a thiol group that attaches well to the Au NPs and an $\mathrm{NH}_{2}$ group that, when protonated to $\mathrm{NH}^{3+}$, allows electrostatic attachment of negatively-charged, citrate-coated $\mathrm{Au}$ NSs. The thiol place-exchange occurs preferentially at vertex/edge sites of the NPs due to less steric hindrance at those sites, which leads to attachment of 4-ATP and subsequent binding of Au NSs to those sites. Figure 2.6 shows a schematic illustration of the attachment of the $25 \mathrm{~nm} \mathrm{Au} \mathrm{NSs}$ on the Au NP via ligand place-exchange between HT and 4-ATP. 

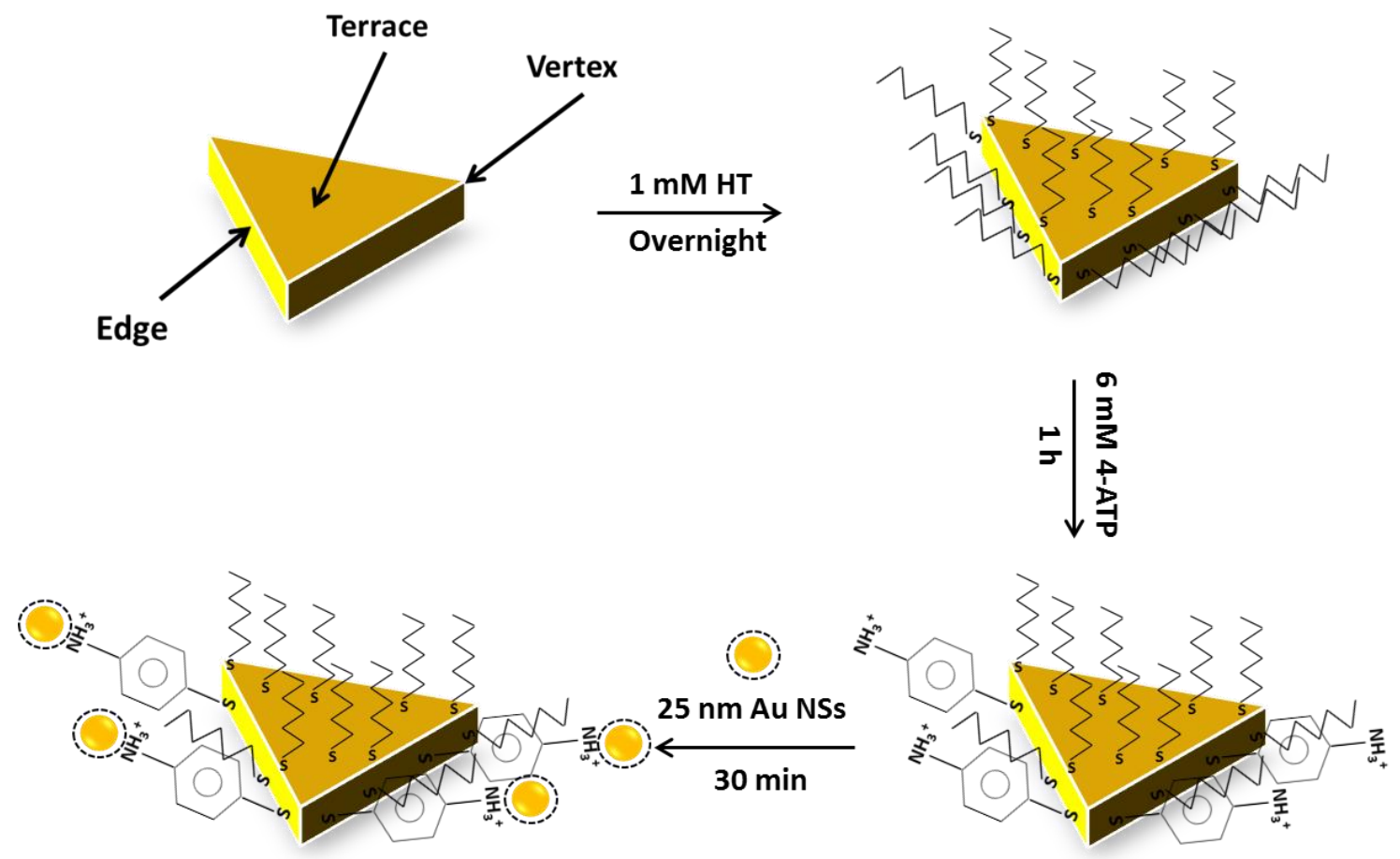

Figure 2.6 A schematic illustration of the attachment of $25 \mathrm{~nm} \mathrm{Au} \mathrm{NSs} \mathrm{onto} \mathrm{the} \mathrm{vertex} \mathrm{and} \mathrm{edge}$ sites of Au NPs via ligand place-exchange reaction between HT and 4-ATP for $1 \mathrm{~h}$. 


\subsection{Controlled Attachment of Au NSs to Terrace Sites of Au NPs}

In order to selectively bind the NSs to terrace sites, the samples were first placed in a $1 \mathrm{mM}$ ethanol solution of 4-ATP overnight, rinsed thoroughly with ethanol, and dried under $\mathrm{N}_{2}$. Then, we exchanged the 4-ATP with HT by placing the sample into a $6 \mathrm{mM}$ ethanol solution of HT for $4 \mathrm{~h}$. We again rinsed thoroughly with ethanol and dried under $\mathrm{N}_{2}$. Finally, the substrates were immersed in $10 \mathrm{~mL}$ of a $\mathrm{pH} 4.8$ solution of $\sim 25 \mathrm{~nm}$ diameter Au NSs for 30 min, rinsed thoroughly with Nanopure ${ }^{\circledR}$ water, and dried under $\mathrm{N}_{2}$. In this procedure, the original 4-ATP ligands on the Au NPs were displaced by HT preferentially at the side face/vertex sites, leading to the blocking of those regions from $\mathrm{Au}$ NS attachment and controlled binding of Au NSs to the terrace sites. Figure 2.7 shows a schematic illustration of the attachment of $25 \mathrm{~nm} \mathrm{Au} \mathrm{NSs} \mathrm{onto} \mathrm{the} \mathrm{Au} \mathrm{NP} \mathrm{via}$ ligand place-exchange of 4-ATP with HT.

\subsection{Performing SEM on Glass Substrates}

DFM images and LSPR spectra require transparent substrates such as glass. However, obtaining high resolution SEM images of metal nanostructures on a glass substrate is challenging due to the low conductivity of glass. When performing SEM on glass samples, a phenomenon called charging can happen. Substrate charging causes a range of unusual effects including abnormal contrast, image deformation and shift. More badly, a sudden discharge of electrons from a highly charged area may cause a bright flash on the screen. These all make it nearly impossible to capture a uniform image of the glass sample. Figure 2.8 shows a comparison of SEM images on a silicon substrate (A) and on a glass substrate (B) at the same operating conditions. It is clear that there 


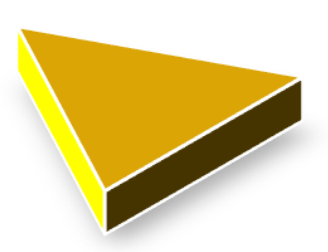

\section{$\underset{\text { Overnight }}{\stackrel{1 \mathrm{mM} \text { 4-ATP }}{\longrightarrow}}$}
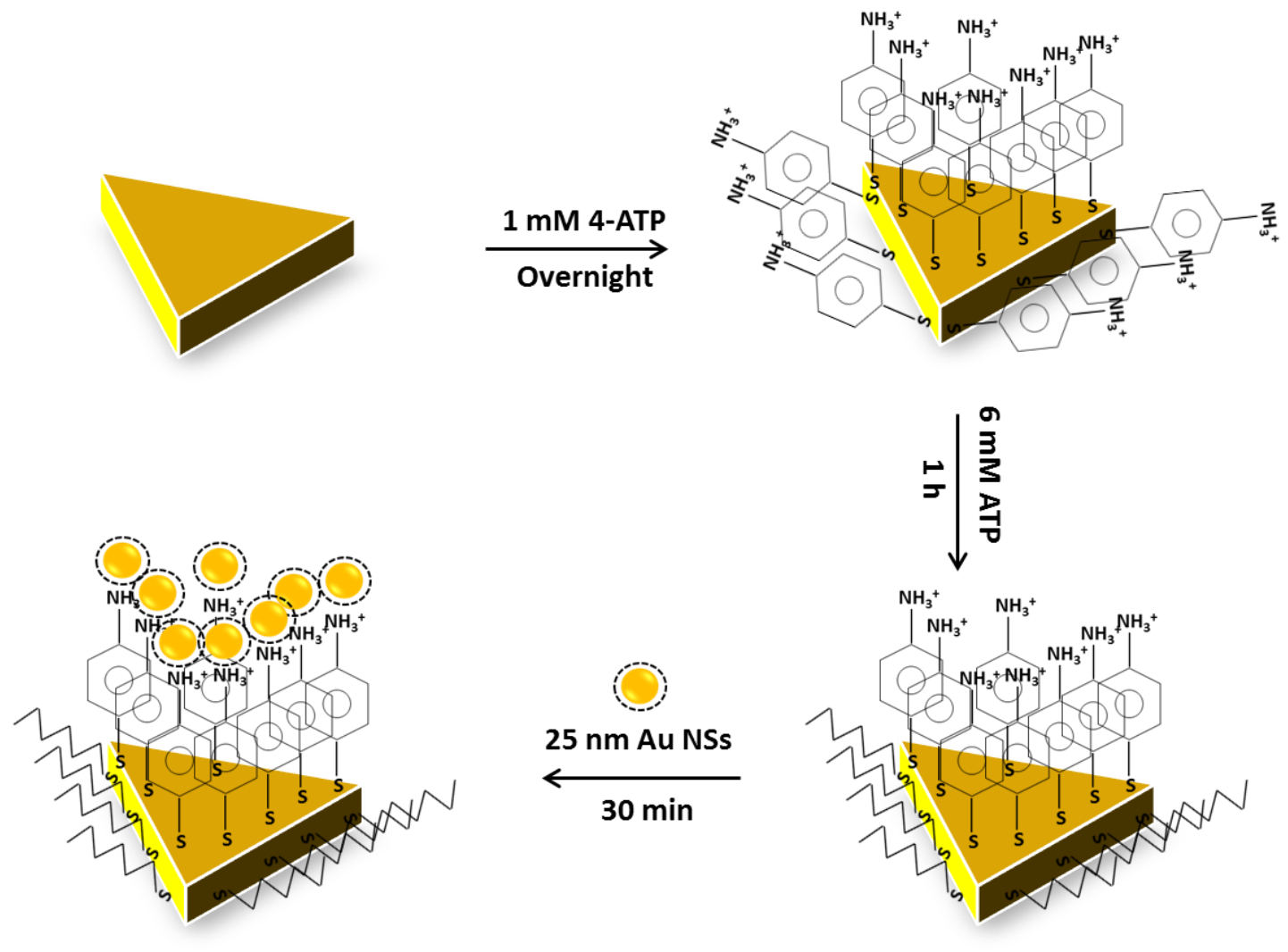

Figure 2.7 A schematic illustration of the attachment of $25 \mathrm{~nm} \mathrm{Au} \mathrm{NSs} \mathrm{onto} \mathrm{the} \mathrm{terrace} \mathrm{sites} \mathrm{of}$ Au NPs via ligand place-exchange reaction between 4-ATP and HT for $1 \mathrm{~h}$. 

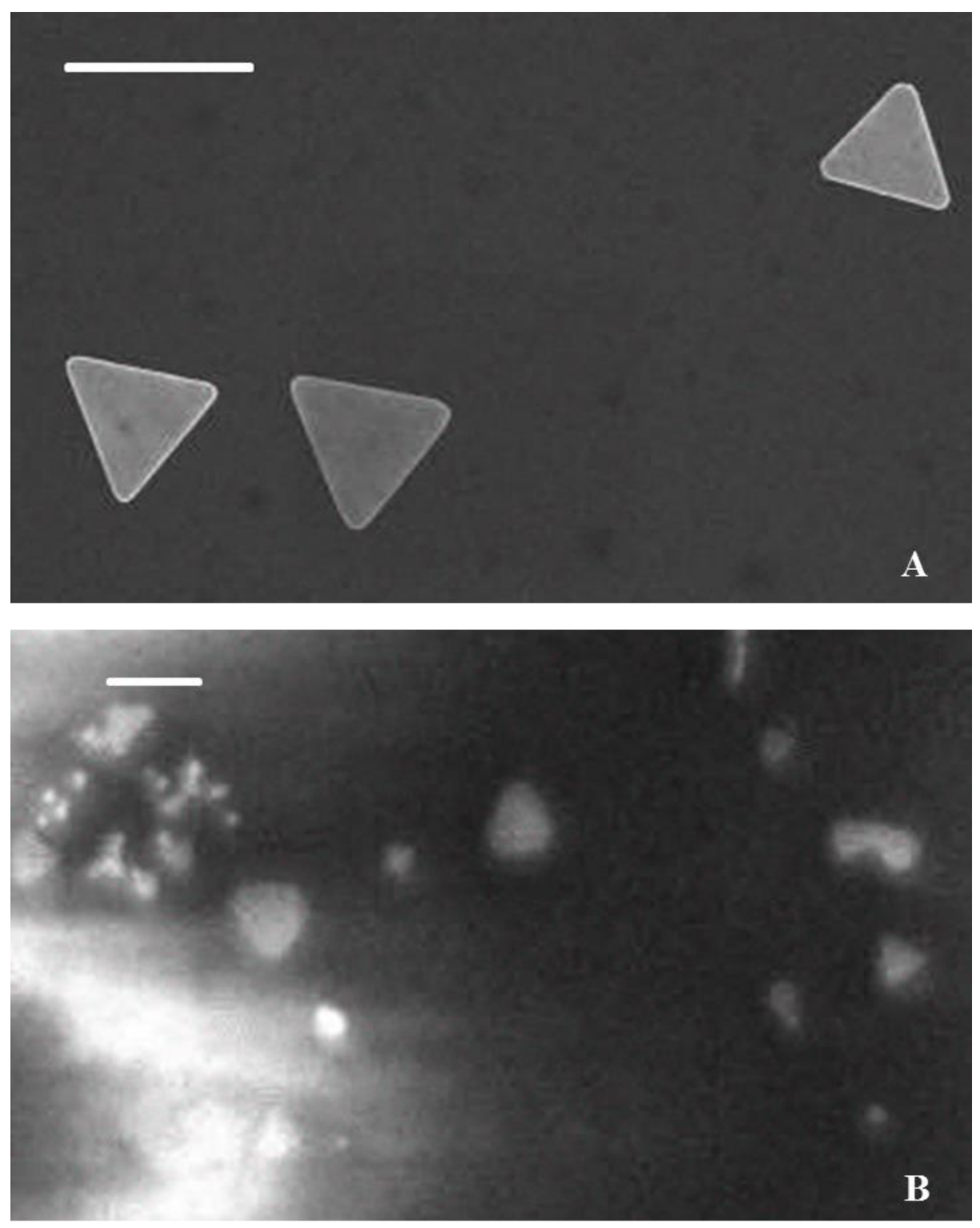

Figure 2.8 SEM images of Au NPs on silicon substrate (A) and a glass substrate (B). Scale bars in SEM images are $500 \mathrm{~nm}$. 
was a very bad charging problem with the glass substrate as well as abnormal contrast. The charging problem occurs because extra electrons build up negative charge at the point where the electron beam hits the sample. The level of charge is related to the energy of the electrons and the number of electrons. The energy of the electrons is determined by the acceleration voltage, so reducing the acceleration voltage can reduce charging. The number of electrons is determined by several parameters such as beam current, emission level of the gun, spot size, and apertures between the gun and the sample. Reducing charging can also be achieved by reducing the number of electrons by adjusting these parameters.

Figure 2.9 shows the comparison of SEM images of Au NPs on a glass substrate at different acceleration voltages. By reducing the voltage from $15 \mathrm{KV}$ to $1 \mathrm{KV}$, the charging has been greatly reduced. However, the resolution is still not good enough to see small NSs around $10 \mathrm{~nm}$. Further reduction of the acceleration voltage to below 1 $\mathrm{KV}$ is not supported by the current used in SEM. The last option to get high resolution SEM images on glass substrates is to sputter coat a layer of conductive materials such as gold, carbon, or platinum onto the glass. An essential prerequisite of the coating method is that the morphology of the nanostructures has no change before and after coating. Different sputtering times were tested to find the optimal coating thickness which is conductive enough to obtain high resolution SEM images but does not change the morphology of the sample (Figure 2.10). We found that a 1 min sputtering time produces the optimal Pt thickness which allows high resolution SEM images for NSs on glass around $10 \mathrm{~nm}$ (Figure 2.10 E). It has also been confirmed that there were no changes in the NP shape due to Pt coating by comparing test Au NPs (synthesized on Si substrates) before and after the same Pt coating. 

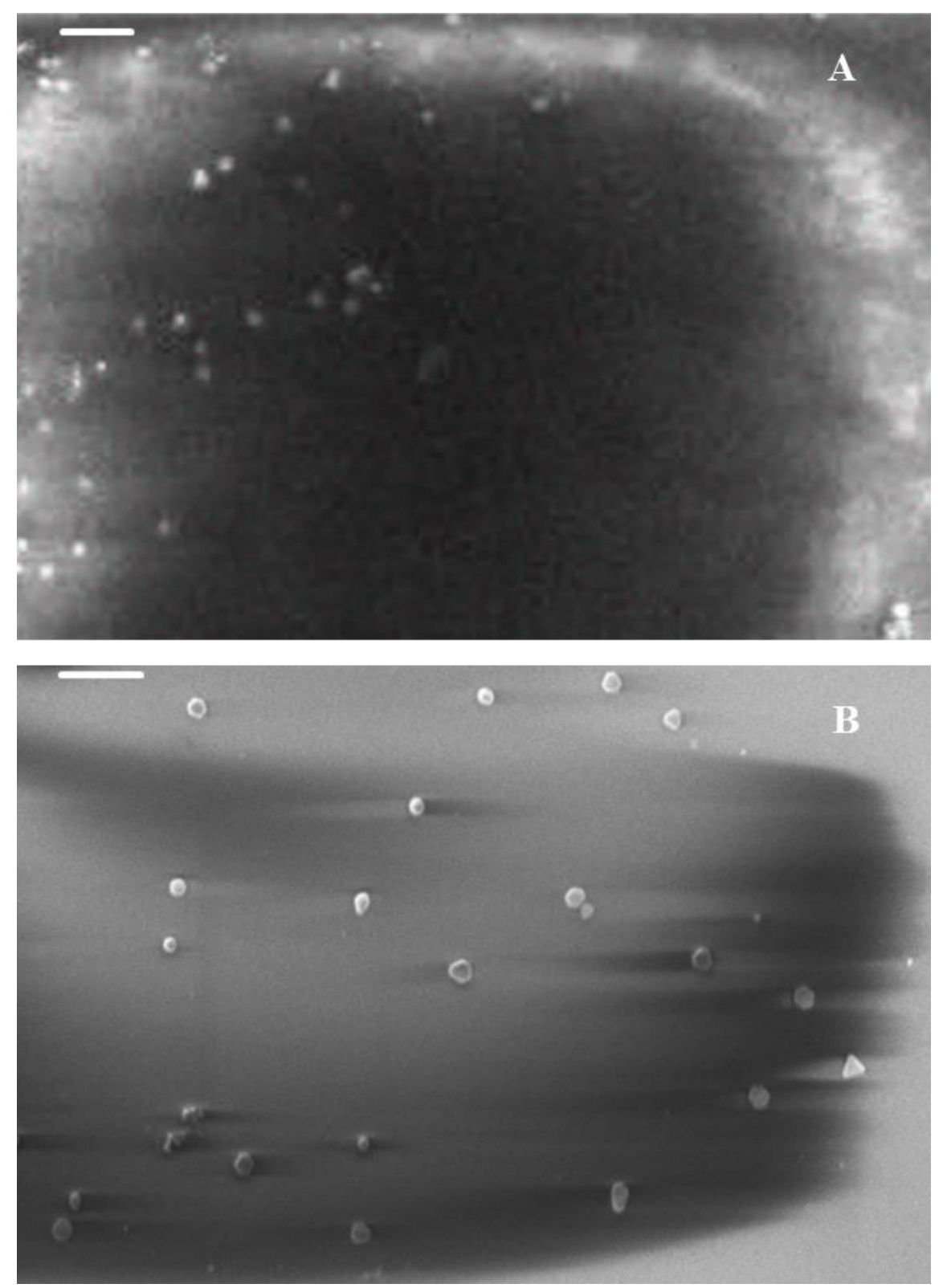

Figure 2.9 SEM images of Au NPs on a glass substrate with SEM acceleration voltage at (A) 15 $\mathrm{KV}$ and (B) $1 \mathrm{KV}$. Scale bars in SEM images are $500 \mathrm{~nm}$. 


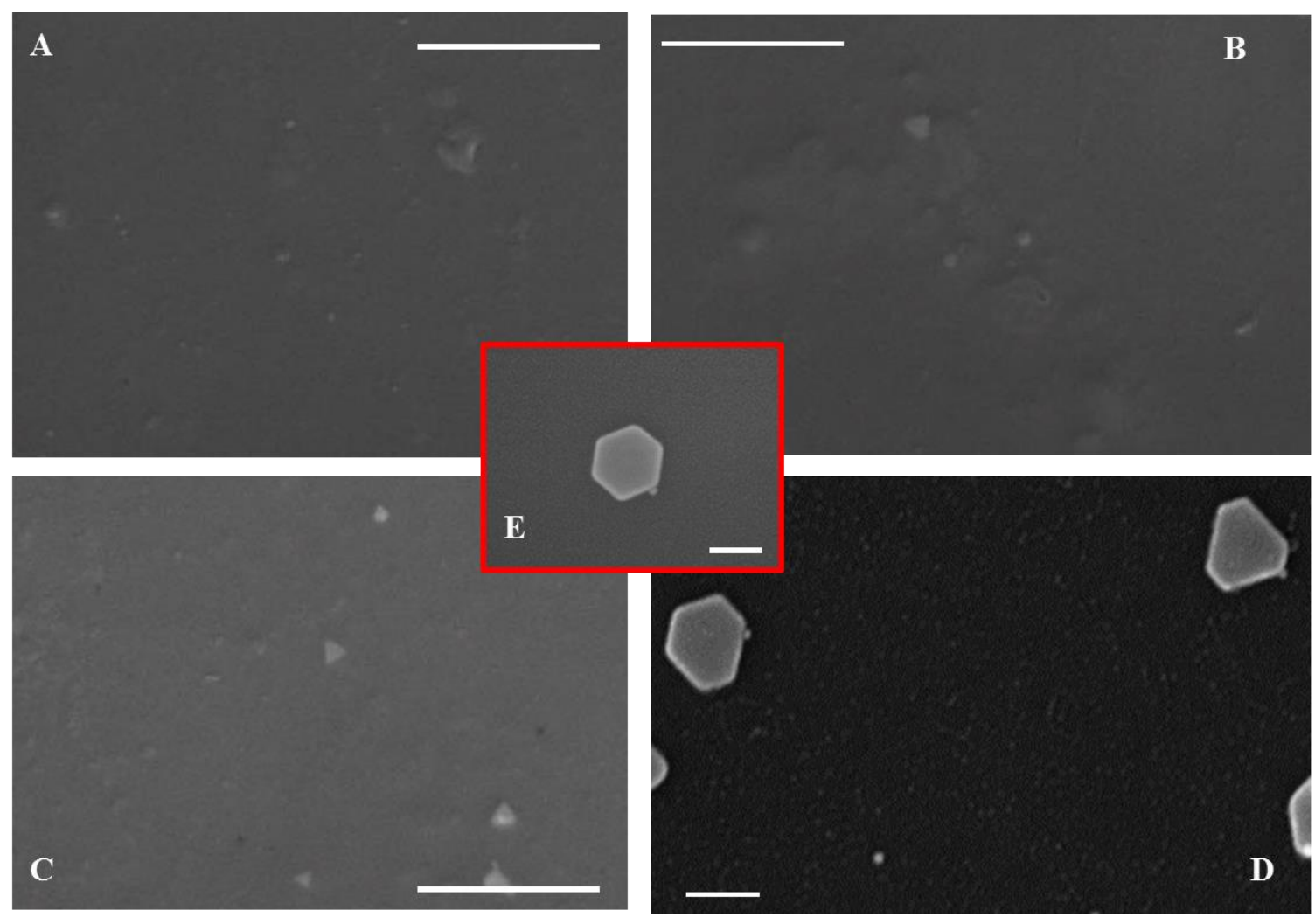

Figure 2.10 SEM images of Au NPs on glass substrates with a Pt coating by sputtering for (A) 10 min, (B) 5min, (C) 3 min, (D) and (E) 1 min. Scale bars in SEM images (A, B, C) are $2 \mu \mathrm{m}$ and in images $(\mathrm{D}, \mathrm{E})$ are $100 \mathrm{~nm}$. 


\subsection{Correlating SEM with LSPR Spectra}

A marker system has been developed to correlate LSPR spectra with SEM images. In a $1.0 \mu \mathrm{m} \times 5.0 \mu \mathrm{m}$ area on glass slides, there are 3700 number markers. Etched numbers on the glass slides were fabricated by photolithography, which was followed by a buffered oxide etch (BOE). First, a photomask was created using the Heidelberg DWL 66FS. Then Fisher brand glass slides were coated with Shipley 1805 photoresist. They were spun at 500 RPM for 5 seconds followed immediately by 4000 RPM for 10 seconds for a final coating of roughly $500 \mathrm{~nm}$ of photoresist. Glass slides were then placed in the SUSS ABM mask aligner for 10 seconds. After mask alignment, glass slides were developed for 1 minute in a MF 319 developer bath and rinsed with DI water. The slides were then dropped in a bath of 1:6 BOE: DI water for 25 seconds to etch the glass. After rinsing with DI water, they were ready for use.

We first synthesized Au NPs directly on glass slides with number markers using the seed-mediated growth procedure described in section 2.6. We then obtained darkfield images of the sample near a number marker (Figure 2.11A). In a defined region near the marker, we collected dark-field scattering spectra for 10-20 individual NPs (Figures 2.11C). After obtaining individual scattering spectra of each NP, we sputtercoated the glass sample with a thin 1-2 nm layer of Pt before obtaining SEM images. Using SEM, the same marker was identified as shown in Figure 2.11B and the same NPs identified near the marker as shown in Figure 2.11D. High-resolution images were obtained for the determination of the size and morphology of each NP. Now the optical information from the DF scattering spectroscopy and the morphology information from SEM were correlated together by those number markers. 

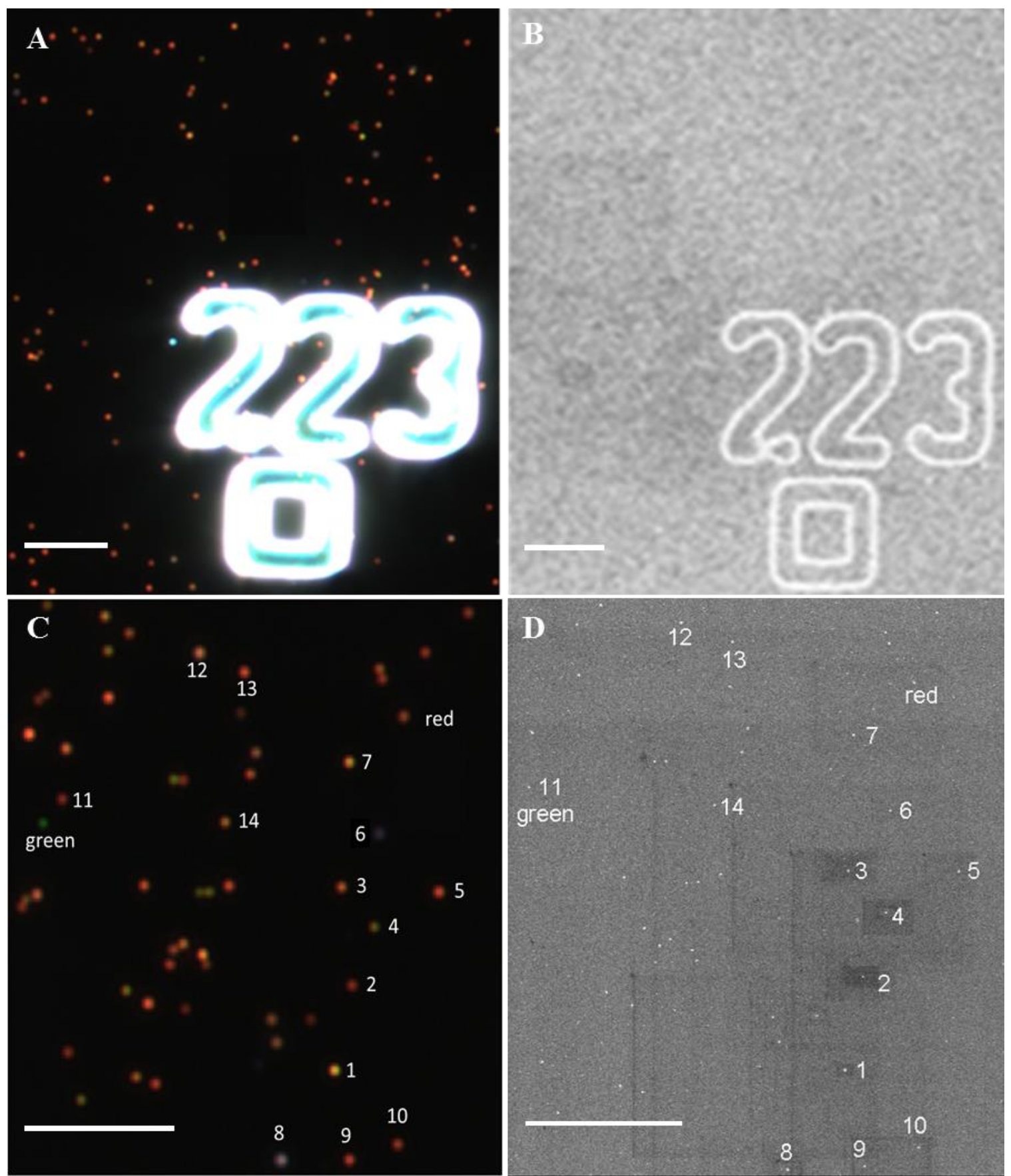

Figure 2.11 Dark-field images (A, C) and correlated SEM images (B, D) of Au NPs on a glass substrate correlated by using the etched marker number 223. Scale bars in DF and SEM images are $10 \mu \mathrm{m}$. 


\subsection{Instrumentations}

\subsubsection{UV-vis Spectroscopy}

Extinction spectra of glass samples were obtained by using a Cary 50 Bio UVVisible spectrophotometer. UV-vis measurements were performed routinely to characterize the growth of $\mathrm{Au}$ nanostructures on surfaces and their optical properties. This provided information about the size and shape of the nanostructures and optical changes during the ligand-exchange reactions.

\subsubsection{Sputter Coating Machine}

In order to obtain high resolution SEM images for nanostructures on marked glass substrates, a thin layer of Pt was coated to increase the conductivity of the glass. This was achieved by sputter deposition using a PS2 Sputtering Unit from International Scientific Instruments Corporation. Different coating times at a fixed current were tested to obtain the optimal thickness for the metal layer.

\subsubsection{Scanning Electron Microscopy (SEM).}

SEM images were obtained using a Carl Zeiss SMT AGSUPRA 35VP field emission scanning electron microscope operating at an accelerating voltage of $8.00 \mathrm{KV}$. SEM images directly provided information about the shape, size, and structure of the NP or NP/NS coupled assemblies. 


\subsubsection{Dark-Field Microscopy/Spectroscopy}

Scattering spectra were collected from individual NPs or coupled NP/NS assemblies using resonant Raleigh dark-field scattering microscopy/spectroscopy. Darkfield images were obtained with an inverted Nikon Eclipse Ti microscope with a halogen lamp light source and a dark-field condenser $(\mathrm{N}=0.95-0.80)$ for sample illumination and a 100x variable aperture oil immersion objective $(\mathrm{N}=0.5-1.3)$ for collection of the scattered light from individual NPs or NP/NS coupled structures. An Infinity camera from Lumenera Corporation was used to obtain high resolution video images for selecting individual particles. The detector used for measuring light scattering intensity as a function of wavelength was a multichannel air-cooled CCD camera used in a Horiba XPLORA-Inv confocal Raman system

\subsubsection{LSPR Spectrum Acquisition and Process}

The following approach was used in order to acquire a single nanoparticle spectrum. First, a certain area with the nanoparticles of interest was imaged and selected (Figure 2.12A). Then LSPR mapping of this area was performed with a fast mode setting including $0.5 \mathrm{~s}$ acquisition times and 1 spectral accumulation (Figure 2.12B). The LSPR map guarantees the accurate location of the nanoparticles of interest. Therefore, an individual nanoparticle was selected on the map and moved to the center of the spectrometer slit. A single LSPR spectrum was collected with a slow mode setting including $5 \mathrm{~s}$ acquisition times and 6 spectral accumulations (Figure 2.13A). This process was repeated for each subsequent nanoparticle of interest. A region where no particles were present was chosen for background subtraction. A single background 

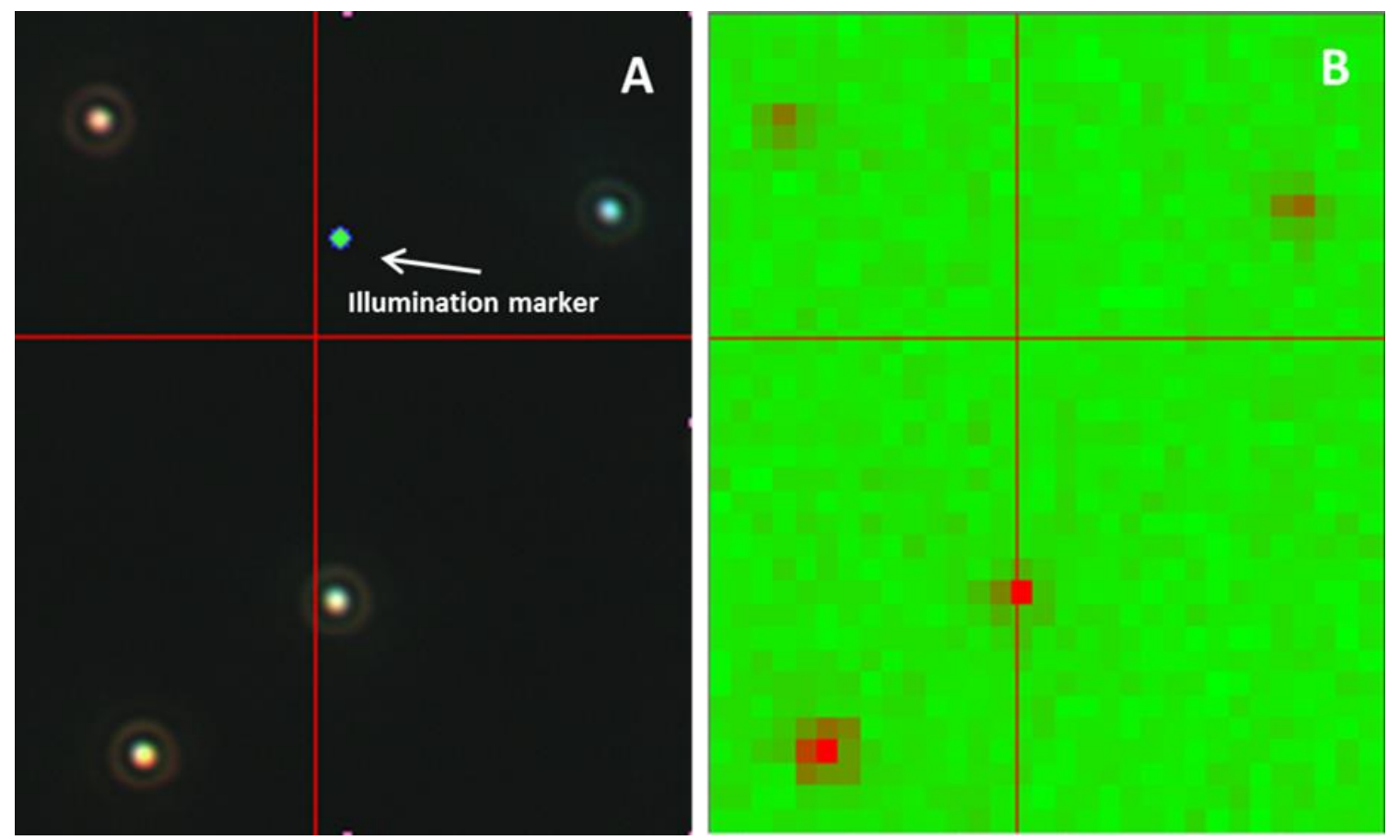

Figure 2.12 A) Dark-field image of a selected sample area with four nanoparticles of interest. B) LSPR map of the same area and corresponding nanoparticles. 


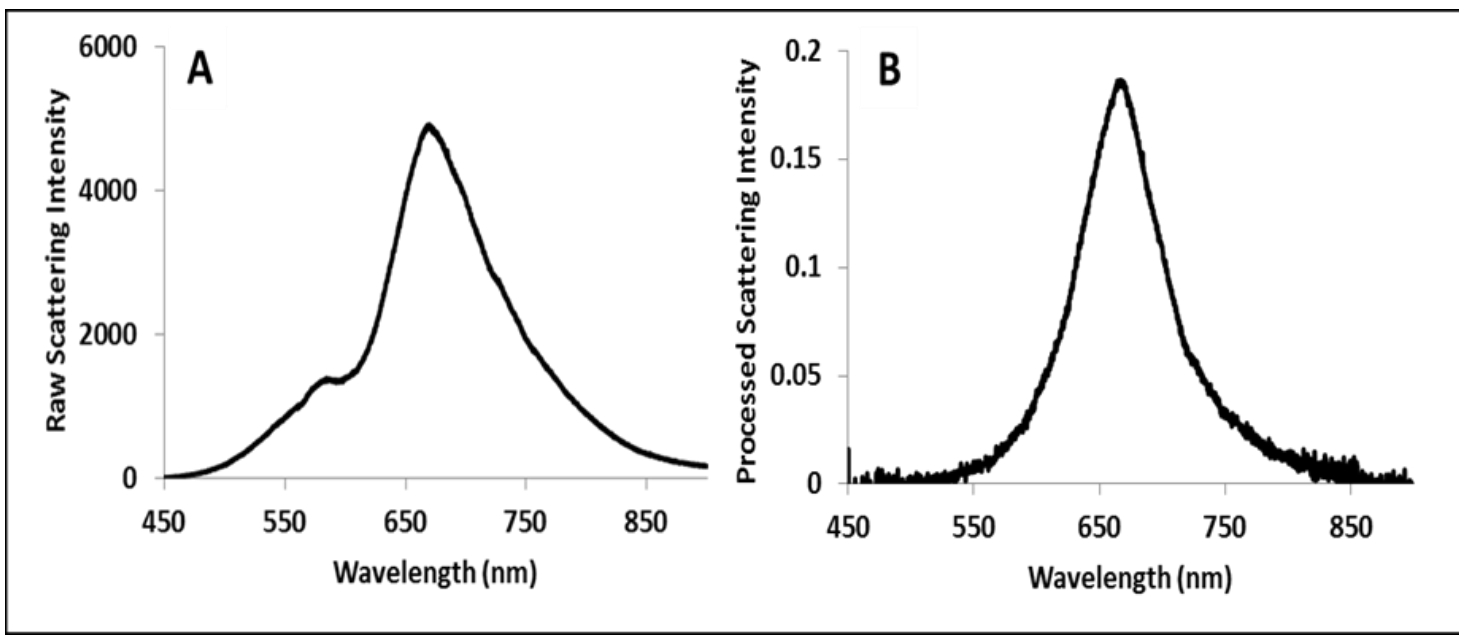

Figure 2.13 A) Raw LSPR spectrum of a single Au nanoplate. B) LSPR spectrum after processing. 
spectrum was also acquired with the same acquisition time and spectral accumulation. These two spectra alone, however, were not sufficient to obtain the true scattering spectrum of a single nanoparticle. The contributions from the wavelength-dependence of the lamp intensity and the detector readout current must be accounted for. For the lamp spectra, the objective NA was increased from 0.5 to 1.3 , such that the directly transmitted light can also be sent to the detector. Care must be taken to not saturate the detector; to this end, short acquisition times were used. The detector dark current was collected with the microscope light turned off. The lamp and detector dark spectra must have the same acquisition parameters, but these parameters may be different than that of the particle and background spectra. With all four spectra available, a single particle spectrum was obtained by subtracting the background spectrum from the raw particle spectrum and then dividing that by the difference between the lamp spectrum and the detector dark current (Figure 2.13B). It is clear that the shoulder peak in the raw particle spectrum disappeared after going through these data processing procedures. The LSPR band was also narrower and sharper than the unprocessed one due to the subtraction of the background and normalization by the lamp spectrum. 


\section{CHAPTER III}

\section{REGIOSELECTIVE PLASMONIC COUPLING IN METAMOLECULAR ANALOGS OF BENZENE DERIVATIVES}

In analogy with benzene-derived molecular structures, we construct plasmonic metamolecules by attaching Au nanospheres (NSs) to specific sites on a hexagonal Au nanoplate (NP). We employ a ligand exchange strategy that allows regioselective control of NS attachment and study resulting structures using correlated electron microscopy/optical spectroscopy at the single-metamolecule level. We find that plasmonic coupling within the resulting assembly is strongly dependent on the structure of the metamolecule, in particular the site of attachment of the NS/s. We also uncover a synergy in the polarizing effect of multiple NSs attached to the NP. Regioselective control of plasmonic properties demonstrated here enables the design of novel structuredependent electromagnetic modes and applications in three-dimensional spatial nanosensors. (Results have been published. Fang et al. Nano Lett., 2015, 15, 542-548) 


\subsection{INTRODUCTION}

Metal nanoparticles have received a lot of attention for their localized surface plasmon resonances (LSPR) for decades; in recent years, however, there has been an upsurge in the work on the construction of complex architectures composed of multiple plasmonic nanoparticles. The level of control and complexity in such assemblies has been constantly improving. The advances in this area can be thought of in the same vein as the synthesis of complex molecules from the chemical bonding of simpler atomic units. This loose analogy is not limited to aspects of the fabrication, but also to the emergence of new optical properties and function in complex architectures. For instance, when two metal nanoparticles are assembled, so as to be in close proximity, their dipolar plasmon oscillations couple via short-range near-fields, often producing new plasmonic modes, which can be of bonding or anti-bonding type, in analogy to concepts of molecular hybridization. ${ }^{80,129,130}$ Dimers of plasmonic NSs, ${ }^{81,131,132}$ nanoshells, ${ }^{82,84}$ nanoprisms, ${ }^{131}$ nanocubes, ${ }^{83}$ and $\mathrm{NRs}^{80}$ and their hybrid plasmon modes have been studied. Bonding plasmon modes, characterized by strongly concentrated electric fields at the dimer junction, ${ }^{131}$ have been exploited for optical sensing, ${ }^{66-69}$ single-molecule detection by surface-enhanced Raman spectroscopy (SERS), ${ }^{70-74}$ and wave-guiding of light. $^{75,76}$

An added level of complexity has been achieved by means of heterodimers of plasmonic nanoparticles, i.e., dimers in which the two nanoparticles are dissimilar in size, shape, or composition. Heterodimers often display new coupling modes and unique spectral features that are not observed with homodimers, ${ }^{2,133}$ including symmetry breaking, appearance of otherwise dark out-of-phase hybrid modes, avoided crossing behavior, Fano resonances, and asymmetric scattering properties. More recently larger 
clusters of plasmonic nanoparticles ${ }^{134}$ and multi-nanoparticle core-satellite nanostructures ${ }^{91-93,135-137}$ have been fabricated and studied for their plasmonic coupling as well as unique optical and magnetic modes that arise from inter-nanoparticle interactions.

In this chapter, we describe a further advance in the addition of control and complexity to plasmonic architecture design by the construction of plasmonic metamolecules that resemble benzene-derived molecules. These novel assemblies are designed by the controlled attachment of Au NSs to specific sites on the surface of a larger hexagonal $\mathrm{Au} \mathrm{NP}$, where we take advantage of past work on ligand exchange reactions on Au surfaces. $^{21,138}$ We achieve control on both the site of NS attachment and the number of NSs attached. The optical properties of plasmonic metamolecules constructed in this manner are characterized by single-particle dark-field scattering spectroscopy. Plasmonic spectra are correlated to assembly morphologies on the level of individual metamolecules, so as to avoid the loss of useful structure/optical property relationships due to ensemble-averaging over a heterogeneous sample.

In these metamolecules, we find hitherto undescribed regioselective behavior, whereby the plasmonic spectrum of a NP/NS assembly is strongly dependent on the site of NS attachment. NSs attached to vertices of the hexagonal NP couple most strongly to the NP, greatly perturbing its native LSPR response; whereas other sites are found to offer weaker electromagnetic coupling. The number of attached NSs and the relative arrangement of NSs are also found to influence the plasmonic response. In particular, a positive synergy is found in the polarizing effect of multiple NSs. In addition, asymmetric distributions of multiple NSs attached to the sides of the NP result in complex, broadened, multi-peaked spectra with large plasmonic shifts. Thus, we demonstrate, in analogy to the molecular realm, that structural complexity on the 
nanoscale leads to new properties. The kind of regio-selective control and behavior described here has utility for the discovery of new emergent optical properties and the design of three-dimensional spatial nanosensors. ${ }^{134}$

\subsection{EXPERIMENTAL}

The procedures of synthesis of the Au NPs and Au NSs, the preparation of nanostructure assemblies via ligand-exchange reactions, and characterization by LSPR and SEM are described in Chapter II.

\subsection{SIMULATION OF LSPR SPECTRUM}

All LSPR spectrum simulation was performed by Jain group from the University of Illinois at Urbana-Champaign. The discrete dipole approximation (DDA) method was used for the simulation of LSPR spectra of Au NPs and for studying plasmon coupling of Au NPs with Au NSs. The DDA method numerically solves Maxwell's equations for one or a series of arbitrarily shaped objects by discretizing each object into a cubic array of $\mathrm{N}$ polarizable point dipoles and self consistently solving for the polarizability of each dipole interacting with the incident field and all other $\mathrm{N}-1$ dipoles. We employed the open source DDSCAT 7.3.0 code from Draine and Flatau.

The hexagonal NP target was generated via tools available on nanoHUB.org. A triangular mesh description of the hexagonal geometry was generated using the opensource 3D design tool "Blender". Using the "DDSCAT Convert" tool (doi: 10.4231/D3FB4WM99), the geometry was discretized into a three-dimensional virtual 
dipole array, the input for DDSCAT. To simulate the dielectric properties of the ligand shell of NPs with Au NSs attached at the side faces/vertices, a 1-nm thick layer of HT (n $=1.448)^{139}$ was added to the surfaces of the top and bottom NP terraces and a 1-nm thick layer of 4-ATP $(n=1.663)^{140}$ was added to the surfaces of the side faces. Coupling between hexagonal NPs and NSs was simulated by attaching NSs to various sites on the side faces/vertices of the NP. To simulate the dielectric properties of the ligand shell of NPs with Au NSs attached at the terraces, a 1-nm thick layer of HT $(n=1.448)$ was added to the surfaces of the side faces and a 1-nm thick layer of 4-ATP $(n=1.663)$ was added to the surfaces of the top and bottom terraces. Au NSs of 27-nm diameter were generated using the DDSCAT subroutine CALLTARGET and combined with the NP target. For every case, the surface-to-surface separation distance between the NP and the NS was 1 $\mathrm{nm}$, i.e., the NS was in contact with the ligand shell, which is the likely scenario in experiments. Note that this interparticle distance is measured along the thickness direction for NSs placed on the terraces and along one of the hexagonal axes for NSs placed on the side faces/vertices.

In order to match experimental conditions of dark-field scattering spectroscopy, all target structures were excited with unpolarized light, simulated by a circularly polarized plane wave, unless otherwise noted. Light, incident normal to the face of the NP, propagated along the thickness of the NP. In all simulations, the refractive index of the ambient medium was set at $\mathrm{nm}=1.1$, representative of a mixture of air $(\mathrm{n} \approx 1)$ and glass $(\mathrm{n} \approx 1.5)$. The relative contribution of air and glass was determined by the relative volume fraction of each within the surrounding medium, the surrounding medium being defined as the additional volume when the NP dimensions were extended 50\% from the 
center of the NP along each axis. The bulk experimental dielectric function of Au from Johnson and Christy was utilized for all calculations without any corrections. ${ }^{141}$

Scattering spectra (scattering efficiency vs. wavelength) were obtained from each simulation. Spectra were plotted in energy units $(\mathrm{eV})$ and then fit in OriginLab to single or multiple Lorentzian peaks, as noted. Peak maxima of scattering bands obtained in energy units $(\mathrm{eV})$ were converted to wavelength units $(\mathrm{nm})$. Standard error was ca. $1 \mathrm{~nm}$ for all Lorentzian fits. Coupling-induced LSPR shifts $\left(\Delta \lambda_{\max }\right)$ were determined from wavelength maxima.

In addition to spectra, for four of the targets (a single NP, a NP coupled with a single NS for two different interparticle distances, and a NP coupled with four NSs), near-field calculations were performed for specific light excitation conditions, i.e., wavelength and polarization. These near-field calculations utilized DDSCAT 7.3 subroutine NEARFIELD. The macroscopic field amplitude $|\mathrm{E}|$ at each grid-point of a volume surrounding the target structure was obtained relative to the incident field amplitude $\left|E_{0}\right|$, with the latter set to a value of 1 . Near-field enhancements $\left(|E| /\left|E_{0}\right|\right)$ were plotted over the surface of the target, using the open source application ParaView.

\subsection{RESULTS AND DISCUSSION}

\subsubsection{Correlation of LSPR Spectrum with SEM Images.}

The experimental strategy used for this study is shown in Figure 3.1. We synthesized Au NPs directly on glass slides using a small modification of our previously

described seed-mediated growth procedure. ${ }^{21,52,142}$ This procedure helped us achieve an 
optimal density and size ( $\sim 100 \mathrm{~nm}$ wide and $30 \mathrm{~nm}$ thick) of Au NPs, amenable to darkfield scattering spectroscopy at the single NP level. The glass slides contained etched numerical markers which were used to correlate dark-field scattering spectra of single NPs with scanning electron microscopy (SEM) images of the same NP. As shown in Figure 3.1A, B, we first obtained dark-field images of the sample near a number marker. We next obtained a dark-field scattering spectrum of 15-20 individual NPs identified in defined regions near the marker (Figures 3.1C and D). After obtaining individual scattering spectra of each NP, we sputter-coated the glass sample with a thin 1-2 nm layer of Pt before obtaining SEM images. We confirmed that there were no changes in the NP shape due to Pt coating by comparing test Au NPs (synthesized on Si substrates) before and after the same Pt coating. Using SEM, the same NPs were identified (Figure 3.1E) and high-resolution images were obtained for the determination of the size and morphology of each NP (Figure 3.1F). In a few cases, we obtained AFM images directly after acquiring light-scattering spectra to determine the NP thickness. In cases where we attached NSs to NPs, we obtained light-scattering spectra of 15-20 NPs before and after NS attachment and obtained SEM images after the attachment.

\subsubsection{Experimental Comparison of Terrace Site vs Edge Site Attachment}

NPs have flat terraces and sharp edge and vertex sites. The attachment of a NS on a terrace (flat surface) and on edge or vertex (sharp region) can affect NP spectrum differently. To investigate this difference, we selectively attached NSs on the terrace site of a NP and on edge site of a NP. SEM images (Figure 3.2) show the successful achievement of regioselective attachment of NSs on the NP, which we exploited for 

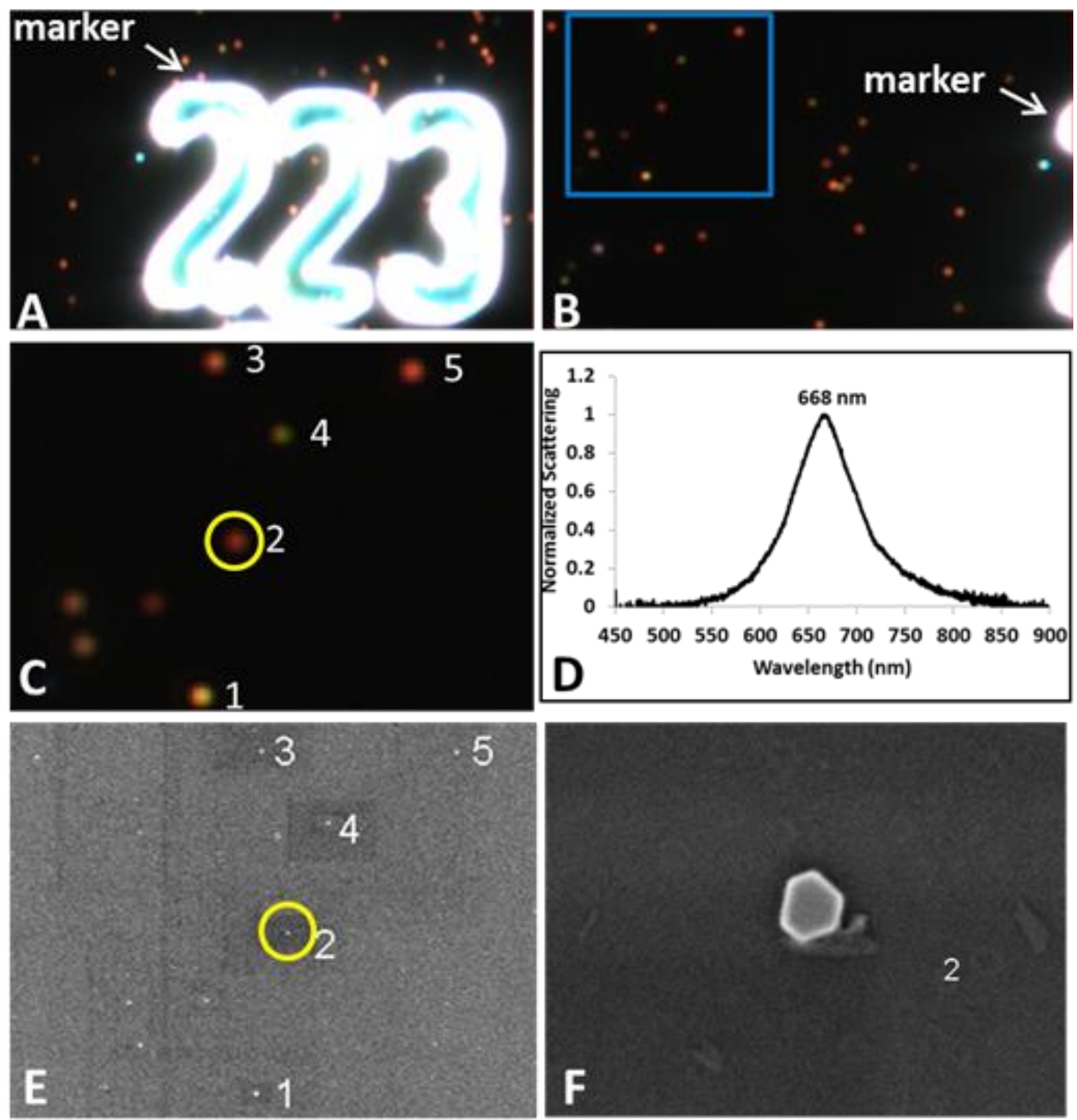

Figure 3.1 Correlated single-nanoparticle dark-field spectroscopy/ electron microscopy. Darkfield microscopy image of Au NP scatterers on a glass slide in the region of an etched marker labeled '223' (A) and in a region directly to the left of the marker (B). The region marked by the blue rectangle in (B) is shown at a higher magnification in (C) with five NPs selected and numbered. The light-scattering spectrum of the Au NP labeled 2 is shown in (D). The spectrum shows a wavelength maximum, $\lambda_{\max }$, of $668 \mathrm{~nm}$. An SEM image of the same region as (C) is shown in (E). A higher magnification SEM image of the NP labeled 2 is shown in (F). 
studying the influence of the attachment site on the plasmonic response of the resulting metamolecule. We see a marked difference between the behaviors for attachment to terraces versus sides (Figure 3.2). For instance, a representative single NP with an initial $\lambda_{\max }$ of $660 \mathrm{~nm}$, upon attachment of an Au NS at a terrace site on the NP, shows a modest red-shift of its $\lambda_{\max }$ by $4 \mathrm{~nm}$. A part of this shift $(1-2 \mathrm{~nm}$ as indicated by control experiments without Au NSs) may simply be a result of an increase in the local refractive index caused by the binding of mixed HT/4-ATP monolayers. Thus, the Au NP has only weak, if any, plasmon coupling with a NS attached to the terrace. On the other hand, another Au NP with an initial $\lambda_{\max }$ of $678 \mathrm{~nm}$ shows a large $21-\mathrm{nm}$ red-shift after the attachment of a NS to one of the vertices on the sides of the NP. This red-shift is indicative of strong plasmon coupling of a NP with a vertex-attached NS.

In order to confirm that this site-dependence of plasmon coupling is indeed statistically significant, we performed the same analysis on five other NPs with Au NSs attached to the terrace region and three other NPs with Au NSs attached to the vertices/side faces. The Au NS diameter ranged from $22-29 \mathrm{~nm}$. The results are summarized in Tables 1 and 2, respectively. The red-shift in $\lambda_{\max }$ for terrace site attachment ranged from 2-4 nm with an average of $3 \pm 1 \mathrm{~nm}$. On the other hand, for vertex/side face attachment, the red-shift in $\lambda_{\max }$ ranged from 14-29 $\mathrm{nm}$ with an average of $22 \pm 5 \mathrm{~nm}$. The range in the magnitude of shift resulted most likely from heterogeneities like i) binding on different terrace sites (inner terrace vs. closer to an edge) or different sites on the sides (vertex vs. edge of a side face vs. center of a side face), ii) differences in the Au NS diameter, or, iii) differences in the original NP size and shape. Despite these heterogeneities, there is reproducibly an order-of-magnitude larger shift in 

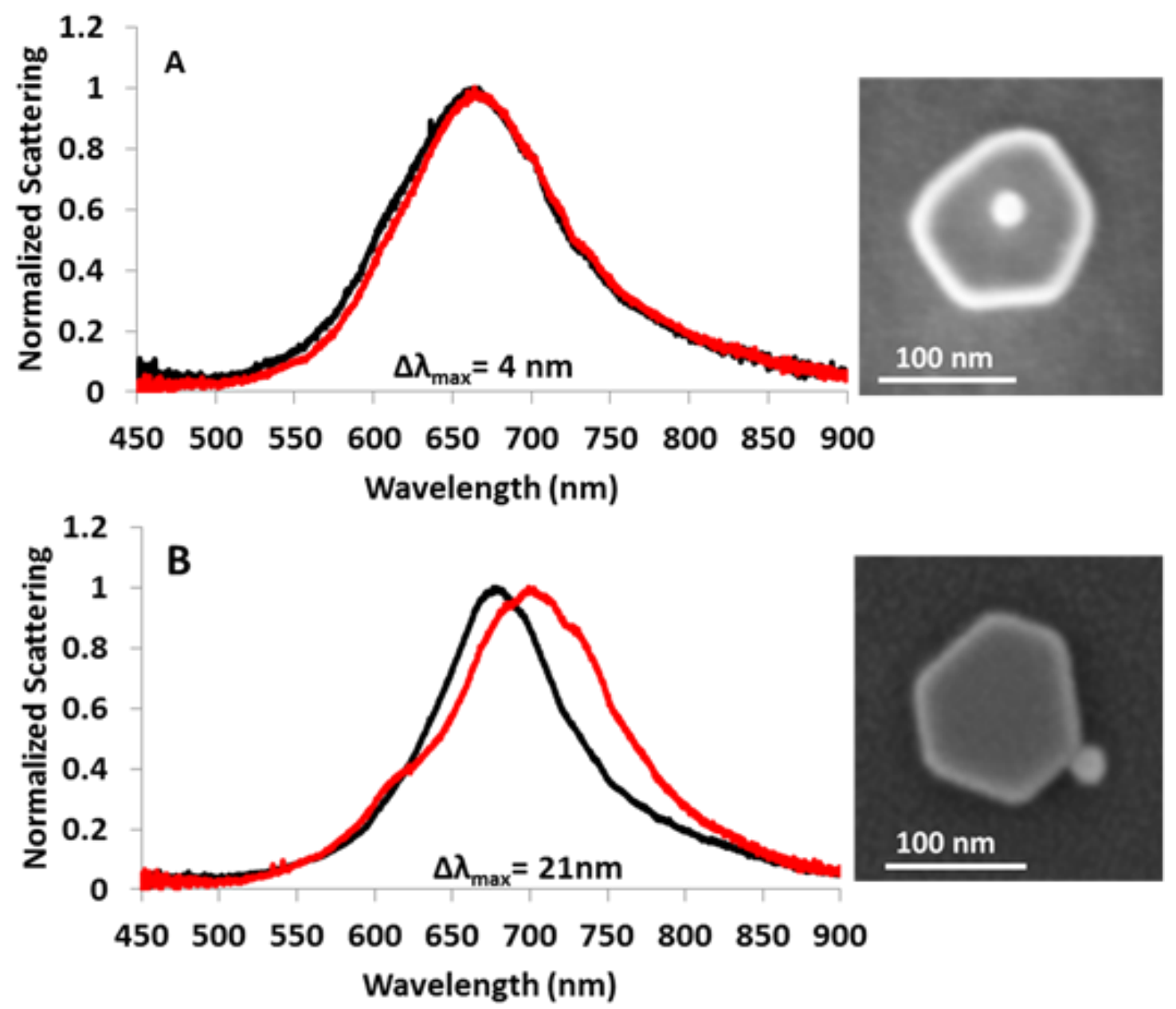

Figure 3.2 Terrace site vs. edge site attachment. (A) Scattering spectrum of a single Au NP before (black line) and after (red line) attachment of a $24 \mathrm{~nm}$ diameter Au NS on an inner terrace site of the NP, as shown in the SEM image to the right. (B) Scattering spectrum of a single Au NP before (black line) and after (red line) attachment of a $27 \mathrm{~nm}$ diameter Au NS on a vertex site, as shown in the SEM image to the right. The $\lambda_{\max }$ values were determined by fitting the spectra to single Lorentzian peaks. The shift in $\lambda_{\max }$ upon NS attachment is indicated. 


\begin{tabular}{|l|l|l|l|l|l|l|}
\hline $\begin{array}{l}\text { Initial } \lambda_{\max } \\
(\mathrm{nm})\end{array}$ & $\begin{array}{l}\text { Initial Peak } \\
\text { Width (nm) }\end{array}$ & $\begin{array}{l}\text { Final } \lambda_{\max } \\
(\mathrm{nm})\end{array}$ & $\begin{array}{l}\text { Final Peak } \\
\text { Width }(\mathrm{nm})\end{array}$ & Shift (nm) & $\begin{array}{l}\text { NS Size } \\
(\mathrm{nm})\end{array}$ & Morphology \\
\hline 657 & 79 & 659 & 82 & 2 & 25 & \\
\hline 659 & 112 & 661 & 104 & 2 & 26 & \\
\hline 660 & 134 & 664 & 124 & 4 & 24 & \\
\hline 679 & 103 & 682 & 105 & 3 & 23 & \\
\hline 686 & 116 & 688 & 123 & 2 & 29 & \\
\hline 707 & 120 & 709 & 117 & 2 & 25 & \\
\hline & & & & $\begin{array}{l}\text { Average } \\
=3 \pm 1\end{array}$ & $\begin{array}{l}\text { Average } \\
=25 \pm 2\end{array}$ \\
\hline
\end{tabular}

Table 3.1. LSPR data and SEM images of representative single Au NPs with a NS attached on the terrace.

\begin{tabular}{|l|l|l|l|l|l|l|}
\hline $\begin{array}{l}\text { Initial } \lambda_{\max } \\
(\mathrm{nm})\end{array}$ & $\begin{array}{l}\text { Initial Peak } \\
\text { Width }(\mathrm{nm})\end{array}$ & $\begin{array}{l}\text { Final } \lambda_{\max } \\
(\mathrm{nm})\end{array}$ & $\begin{array}{l}\text { Final Peak } \\
\text { Width }(\mathrm{nm})\end{array}$ & $\begin{array}{l}\text { Shift } \\
(\mathrm{nm})\end{array}$ & $\begin{array}{l}\text { NS Size } \\
(\mathrm{nm})\end{array}$ & Morphology \\
\hline 653 & 89 & 667 & 133 & 14 & 22 & \\
\hline 673 & 82 & 696 & 88 & 23 & 20 & \\
\hline 673 & 96 & 702 & 120 & 29 & 28 & \\
\hline 678 & 94 & 699 & 138 & 21 & 27 & \\
\hline & & & & $\begin{array}{l}\text { Average } \\
=22 \pm 5\end{array}$ & $\begin{array}{l}\text { Average } \\
=24 \pm 4\end{array}$ & \\
\hline
\end{tabular}

Table 3.2. LSPR data and SEM images of representative single Au NPs with a NS attached on a side face/vertex. 
$\lambda_{\max }$ for vertex/side face attachment as compared to terrace attachment, conclusively indicating the strong dependence of plasmon coupling strength on the attachment location.

\subsubsection{Discrete Dipole Approximation (DDA) Simulation}

\section{Effect of light polarization direction on the LSPR spectrum of a single}

hexagonal NP: An electrodynamic DDA simulation method was used to simulate the LSPR spectrum of a single hexagonal NP. Simulations were performed as described in section 3.3. The experimental NP had a long-axis length of $136 \mathrm{~nm}$ and a thickness of $\sim 33 \mathrm{~nm}$, as estimated by AFM. Simulations were performed for a hexagonal NP with a long-axis length of $137 \mathrm{~nm}$, a thickness of $31 \mathrm{~nm}$, and a 1-nm thick ligand shell of a 50:50 mixture of HT and 4-ATP, which is representative of the experimental structure. Figure 3.3 shows the experimental spectrum and simulated spectrum for a single hexagonal NP. We obtained the $\lambda_{\max }$ values by fitting the spectra to a single Lorentzian peak with a standard error of ca. $1 \mathrm{~nm}$. The simulated spectra also agreed well with the experimental one even though there was $\sim 20 \mathrm{~nm}$ difference, which might be caused by the different sharpness of the NP curvature or the thickness of NP between experiment and simulation. In order to investigate the effect of light polarization direction on the LSPR spectrum, we simulated the spectrum under three conditions, including unpolarized light, linearly polarized light along the long axis and short axis of the NP. The simulated scattering spectra were similar for the different polarization conditions, which is due to the in-plane isotropy of the hexagonal NP. 


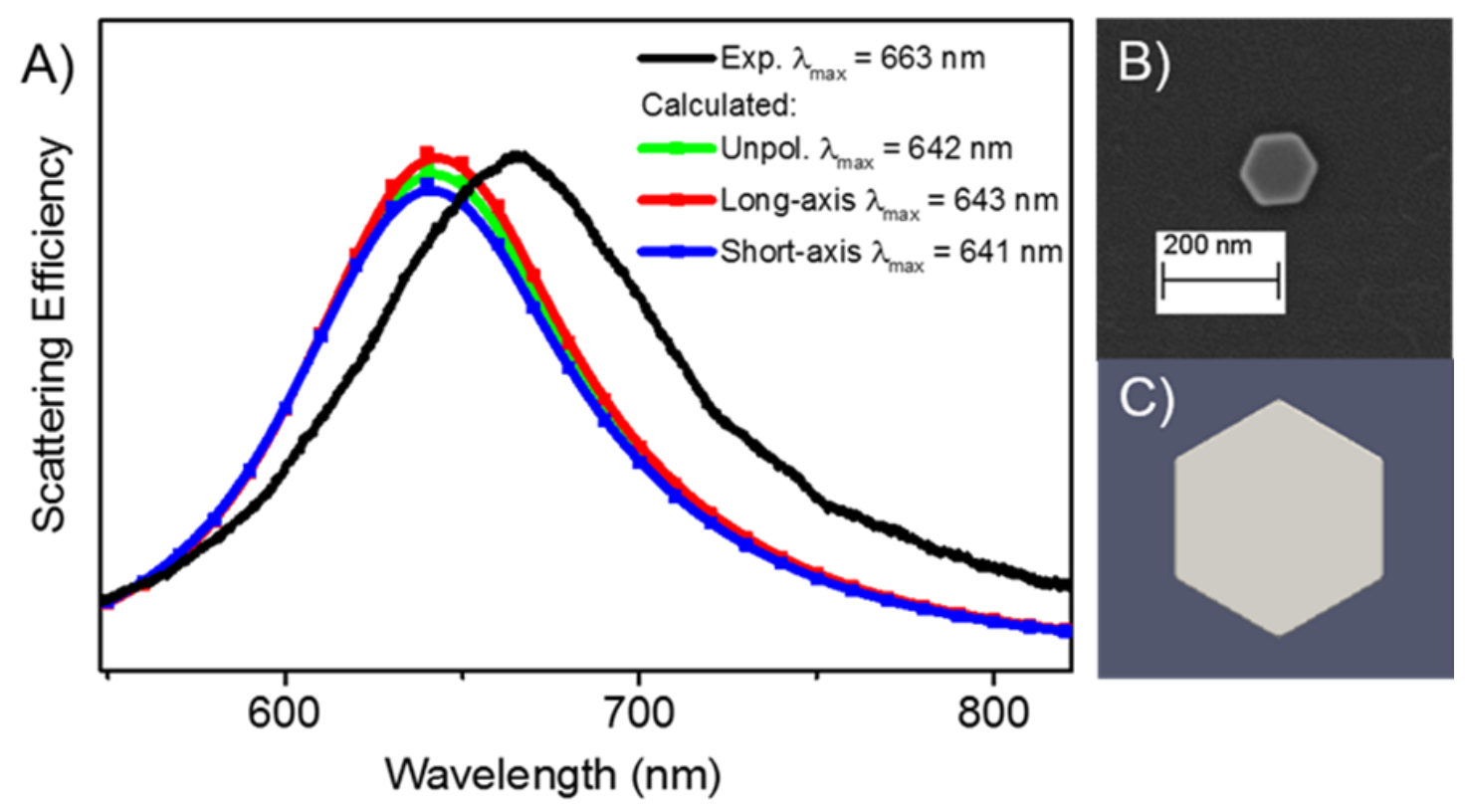

Figure 3.3 Experimental and simulated scattering spectra of a hexagonal Au NP. (A) Experimental scattering spectrum (black curve) of a single hexagonal Au nanoplate obtained via dark-field spectroscopy and DDA simulated scattering spectrum (red, green, and blue curves). (B) SEM image of the experimental NP. (C) Rendition of the simulated structure. 


\section{Effect of light polarization direction on the LSPR spectrum of coupled}

NS/NP: For isotropic nanostructures such as NSs or NPs, their scattering spectra are similar under different polarization conditions. However, the spectra of coupled NS/NP might be different under different polarization directions. The effect of incident light polarization on the plasmon coupling between a Au NP and a Au NS attached to a vertex on the side of the NP has been investigated in Figure 3.4. Simulations were performed for a hexagonal Au NP with a long axis of $137 \mathrm{~nm}$, a thickness of $31 \mathrm{~nm}$, and a 1-nm thick ligand shell of HT and 4-ATP, representative of the experimental structure. A single $\mathrm{Au}$ NS, with a diameter of $27 \mathrm{~nm}$, was attached to a vertex on the side of the NP, in the plane of the hexagon, as shown by the rendition in the inset. The distance between the surfaces of the NP and the NS was $1 \mathrm{~nm}$, measured along the long-axis of the hexagon. The wavelength maxima of scattering were determined by fitting to single Lorentzian peaks. The dashed black line shows the $\lambda_{\max }$ for a NP without an attached NS. Relative to the peak position of this uncoupled case, coupling with the NS gives rise to a red-shift of $23 \mathrm{~nm}$ when the excitation light is polarized along the interparticle axis (red data-points connected by a cubic spline). On the other hand, there is a blue shift of $1 \mathrm{~nm}$ when light is polarized perpendicular to the interparticle axis (blue data-points connected by a cubic spline). This difference is consistent with the excitonic model of LSPR coupling. ${ }^{80}$ In the former case, the dipolar plasmon oscillations of the NP and NS are aligned head-to-tail, resulting in favorable coupling. In the latter case, the dipolar plasmon oscillations of the NP and NS are parallel to one another and therefore coupling between them is weakly repulsive. With unpolarized light excitation (black data-points connected by a cubic spline), the net strength of coupling and the extent of LSPR redshift is intermediate to the two polarization extremes. 


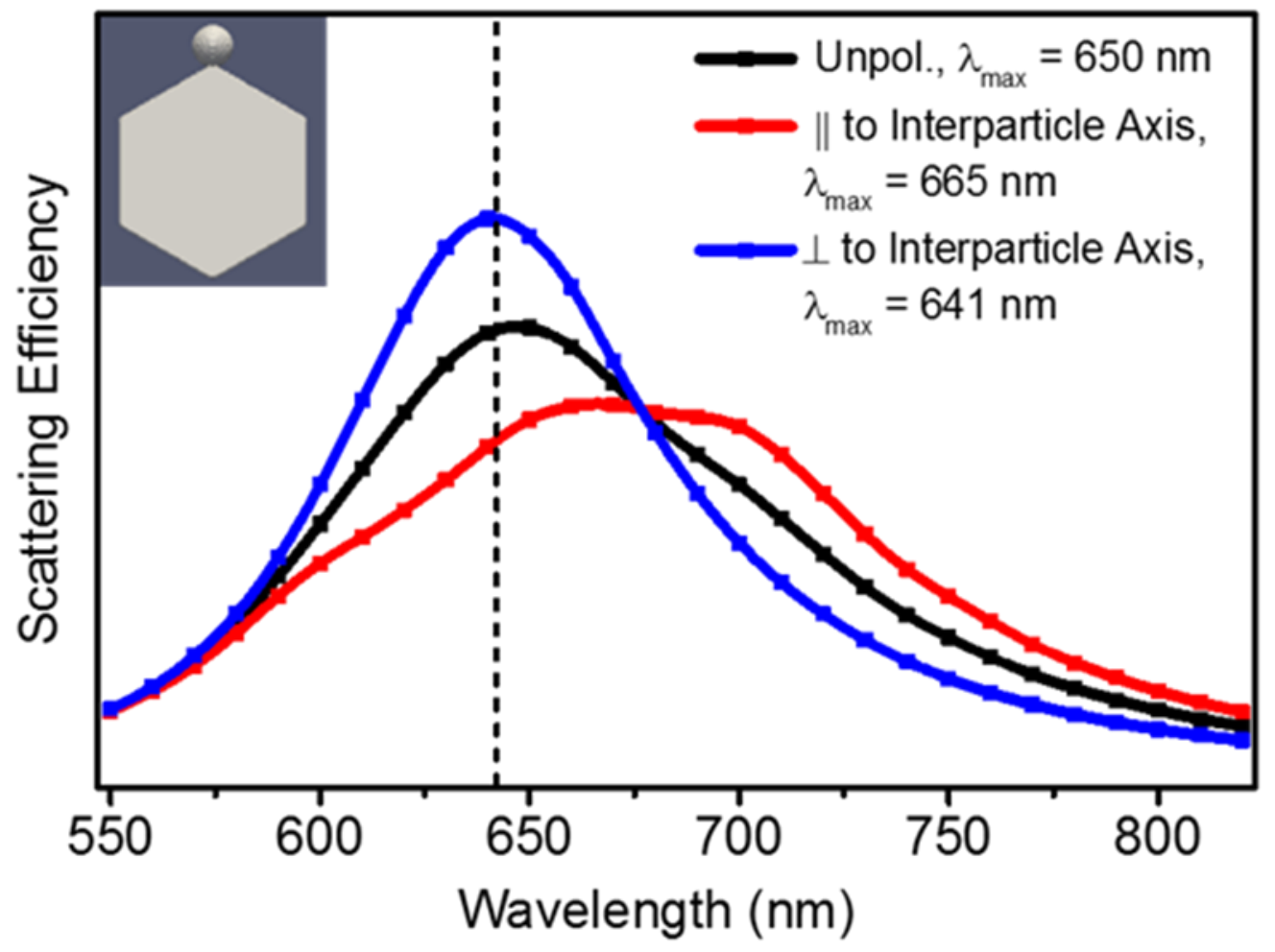

Figure 3.4 Simulated effect of incident light polarization on the plasmon coupling between a $\mathrm{Au}$ NP and a Au NS attached to a vertex on the side of the NP. 


\section{Effect of the attachment location of the NS along the sides of the NP:}

Different regions along the sides of the NP have different strength of electromagnetic field. NSs attached on the different locations along the sides of the NP can cause different amount of LSPR shifts. To investigate this effect, simulations were performed for a hexagonal Au NP with a long axis of $137 \mathrm{~nm}$, a thickness of $31 \mathrm{~nm}$, and a 1-nm thick ligand shell of HT and 4-ATP, representative of the experimental structure as shown in the top panel of Figure 3.5. A single Au NS, $27 \mathrm{~nm}$ in diameter, was then placed at one of four select locations along the sides of the NP: at a vertex in the plane of the hexagon (left, middle panel), at the center of a side face in the plane of the hexagon (right, middle panel), at a vertex out of the plane of the hexagon (left, bottom panel), and at the center of a side face out of the plane of the hexagon (right, bottom panel). Renditions of these simulated configurations are shown besides each spectrum. The surfaces of the NS and the NP were separated by a distance of $1 \mathrm{~nm}$, measured along one of the axes of the hexagon. Excitation was performed with unpolarized light. Spectra are shown as datapoints connected by cubic splines (solid black curves). Wavelength maxima of scattering spectra were determined by fitting to single Lorentzian peaks. The dashed red line shows the $\lambda_{\max }$ for a NP without an attached NS. Relative to the peak position for this uncoupled case, coupling with the NS gives rise to a red-shift, indicated by $\Delta \lambda_{\max }$. Of the cases considered, coupling is strongest $\left(\Delta \lambda_{\max }=8 \mathrm{~nm}\right)$ when the NS is located at the vertex. Coupling is weaker for the cases where the NS is located at the center of a side face, away from the vertices. Also, for the cases where the NS is located on the side face of the NP, a noticeable shoulder emerges in the scattering spectrum; this feature affects somewhat the apparent red-shift obtained from the fit to a single Lorentzian peak. The 
coupling is slightly weaker for the out-of-plane side face location $\left(\Delta \lambda_{\max }=3 \mathrm{~nm}\right)$ as compared to the in-plane side face location $\left(\Delta \lambda_{\max }=4 \mathrm{~nm}\right)$.

Effect of the location of the NS on the terrace of the NP: Similar to the attachment location of the NS along the sides of the NP, the attachment location of a NS on the terrace of the NP might also affect the NS/NP coupling. Simulations were performed for a hexagonal Au NP with a long axis of $137 \mathrm{~nm}$, a thickness of $31 \mathrm{~nm}$, and a 1-nm thick ligand shell of HT and 4-ATP, representative of the experimental structure as shown in the topmost panel in Figure 3.6. A single Au NS, $27 \mathrm{~nm}$ in diameter, was then placed at one of three select locations on the terrace of the NP: at the center of the terrace (second panel from top), close to the edge of the terrace (bottommost panel), and midway between these two locations (third panel from top). Renditions of these simulated configurations are shown besides each spectrum. The surfaces of the NS and the NP were separated by a distance of $1 \mathrm{~nm}$, measured along the thickness of the NP. Excitation was performed with unpolarized light. Spectra are shown as data-points connected by cubic splines (solid curves). Wavelength maxima of scattering spectra were determined by fitting to a single Lorentzian function. The dashed red line shows the $\lambda_{\max }$ for a NP without an attached NS. Relative to the peak position of this uncoupled case, coupling with the NS gives rise to a red-shift, indicated by $\Delta \lambda_{\max }$. For all three positions, the $\Delta \lambda_{\max }$ is $3 \mathrm{~nm}$. Figure 3.8 shows that a terrace NS couples to the surface of the NP but not necessarily to the NP dipolar mode, making the lateral position of the NS in relation to the NP edges less important. For the case where the NS is located at the edge of the terrace (bottommost panel), a noticeable shoulder emerges in the scattering spectrum; this feature affects somewhat the apparent red-shift obtained from the fit to a single Lorentzian peak. 

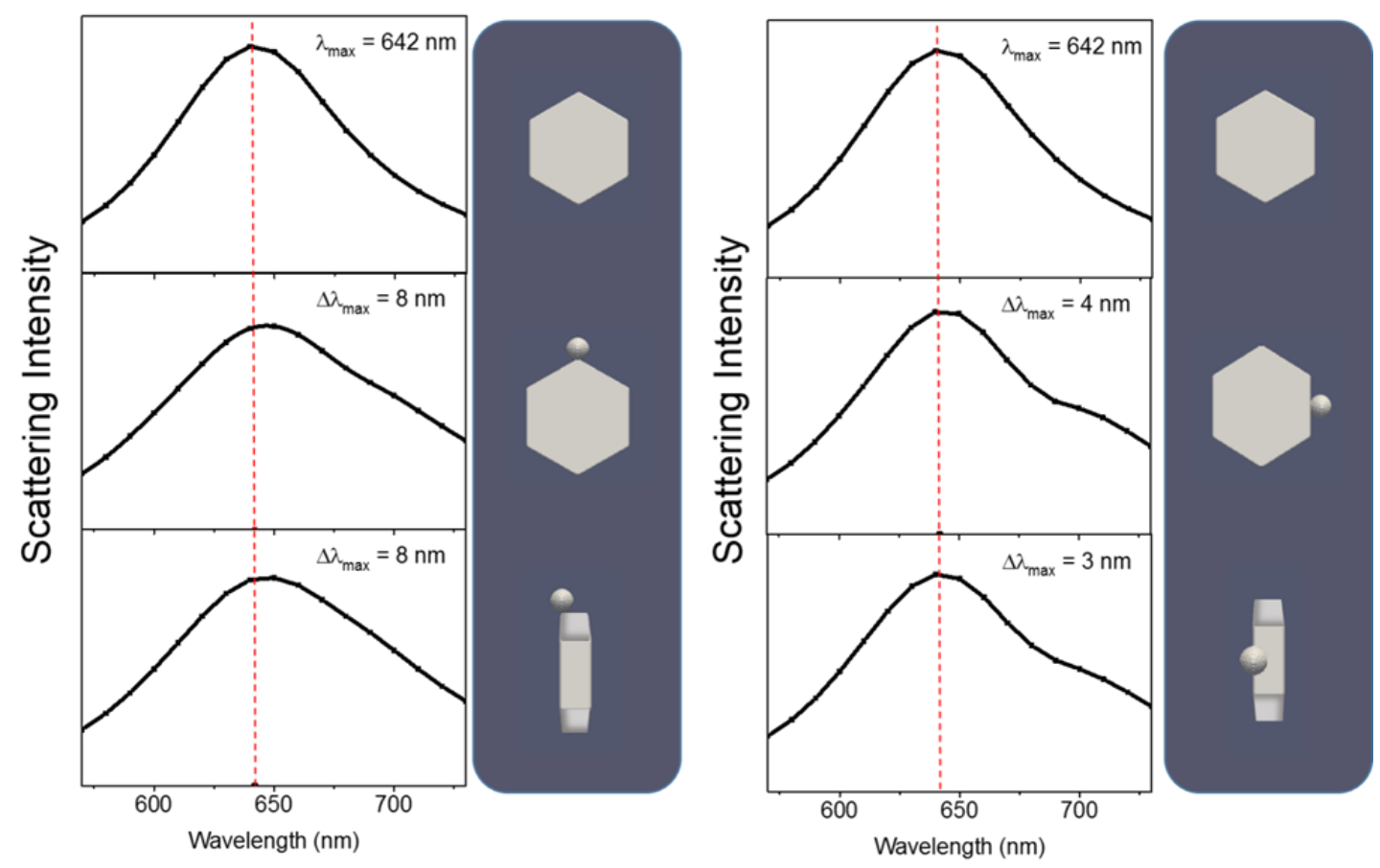

Figure 3.5 Simulated plasmon coupling between a Au NP and a Au NS: effect of the attachment location of the NS along the sides of the NP. 


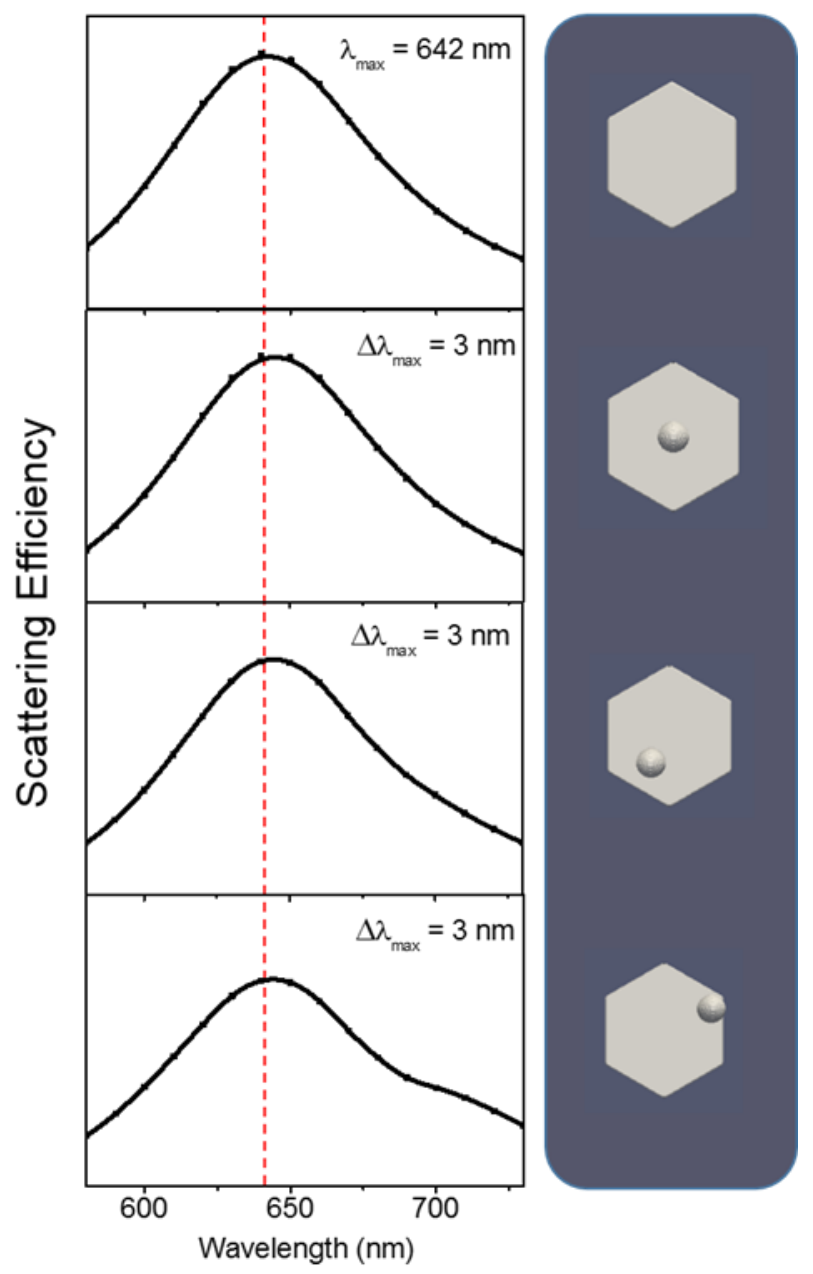

Figure 3.6 Simulated plasmon coupling between a hexagonal Au NP and an Au NS: effect of the location of the NS on the terrace of the NP. 
Effect of the distance between the NS and the NP: Coupling-induced shifts greatly depend on the inter-particle distance. Simulations were performed for a hexagonal $\mathrm{Au} \mathrm{NP}$ with a long axis of $137 \mathrm{~nm}$, a thickness of $31 \mathrm{~nm}$, and a 1-nm thick ligand shell of HT and 4-ATP, representative of the experimental structure in Figure 3.7. A single Au NS, $27 \mathrm{~nm}$ in diameter, was then placed either at the vertex on the side in the plane of the hexagon (A) or at the center of the terrace (B) at varying surface-to-surface interparticle distances. This distance was measured along the NP long-axis for (A) and along the thickness direction for (B). Excitation was performed with unpolarized light. Spectra are shown as data-points connected by cubic splines (solid curves). Wavelength maxima of scattering spectra were determined by fitting to single Lorentzian peaks. The coupling-induced red-shift $\Delta \lambda_{\max }$, relative to the LSPR peak position of a NP without an attached NS, is plotted in (C) as a function of surface-to-surface interparticle distance. For each case, four distances have been simulated including $1 \mathrm{~nm}, 2 \mathrm{~nm}, 5 \mathrm{~nm}$, and 30 $\mathrm{nm}$. The coupling strength, as indicated by the magnitude of the red-shift, increases with decreasing interparticle distance. From the simulated near-field of a Au NP coupled to a Au NS placed on the terrace of the NP, the NS and NP couples stronger at a distance of 1 $\mathrm{nm}$ than $5 \mathrm{~nm}$ (Figure 3.8). For the left image, the surface-to-surface interparticle distance is $5 \mathrm{~nm}$ and for that on the right, it is $1 \mathrm{~nm}$. The corresponding spectra are shown in Figure 3.7. The near-field pattern shows a strong dipolar mode excited in the NP. The NS placed 5-nm away from the NP exhibits an asymmetric near-field distribution: the near-field is concentrated on the NS surface that is closer to the NP. At a distance of 1-nm, the near-field is concentrated in the gap between the NS and NP, which is indicative of an attractive interaction between the dipolar plasmon oscillation of the NS and image dipoles induced on the surface of the NP. 
A)
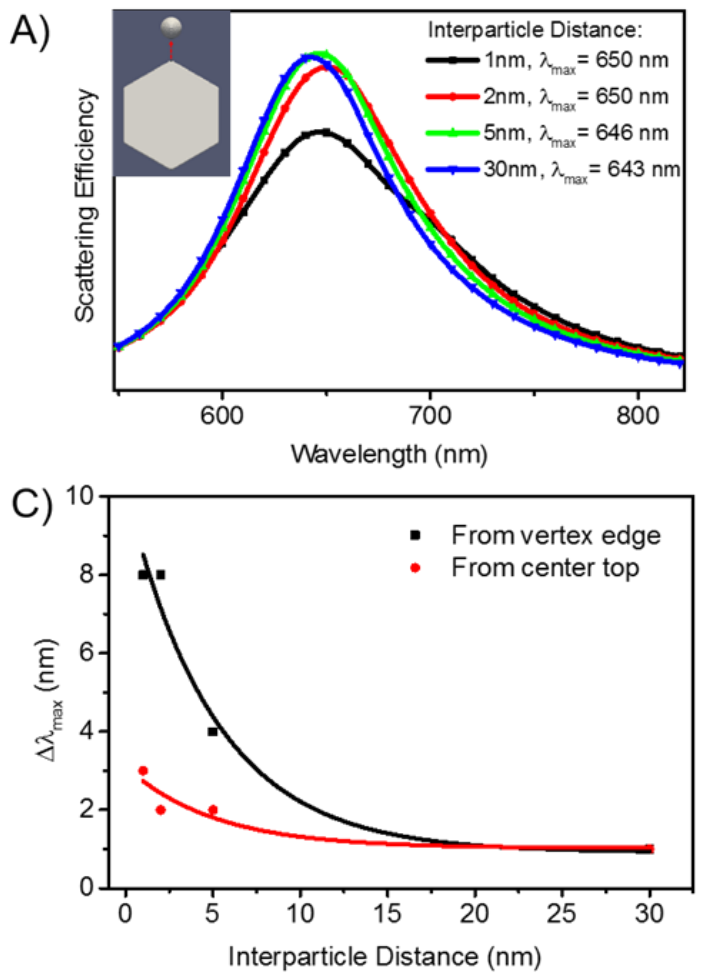

B)

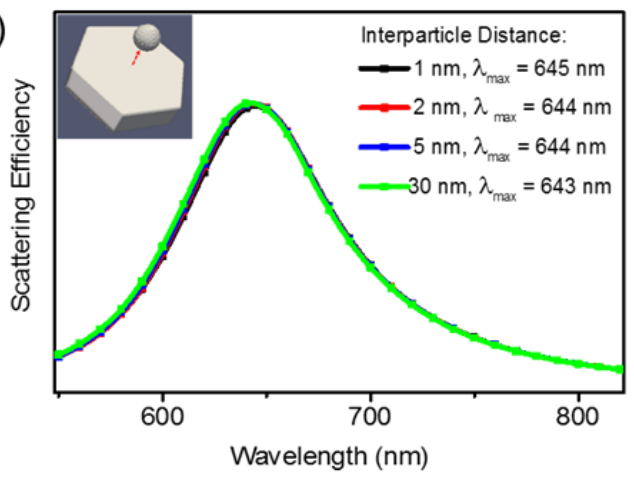

Figure 3.7 Simulated distance-dependence of plasmon coupling between a Au NP and a single Au NS for two cases: a NS placed at a vertex on the side (A) and a NS placed at the center of the terrace (B) as shown by the renditions in the insets. The relationship between inter-particle distance and wavelength shift is shown in (C). 


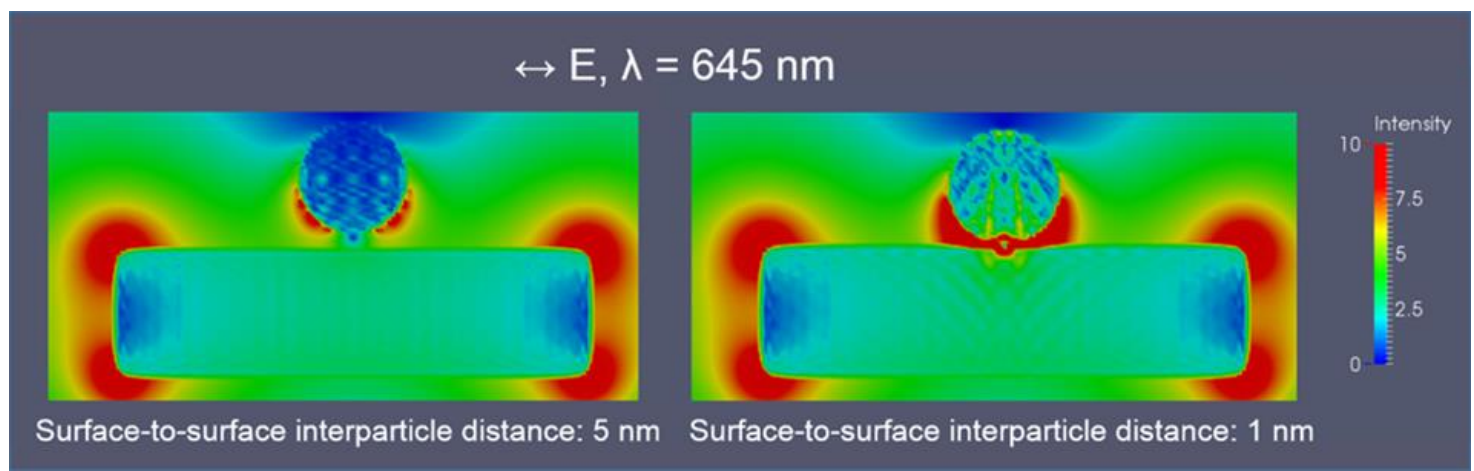

Figure 3.8 Simulated near-field of a Au NP coupled to a Au NS placed on the terrace of the NP. The images represent a side-view of a cross-section taken along the polarization direction. The near-field is depicted in the form of an amplitude enhancement $\left(|\mathrm{E}| /\left|\mathrm{E}_{0}\right|\right)$ on a color scale. 
Simulation of terrace site vs. edge site attachment: After all the simulation conditions have been investigated, it is time to simulate the terrace site and edge site attachment and make a comparison with experiment results. DDA simulation confirmed the observed regioselective nature of plasmon coupling in NP/NS dimers (Figure 3.9). Simulations show that the attachment of a Au NS to a vertex on the side of the NP results in a 8-nm LSPR red-shift with respect to the LSPR of a NP with no NS attached. In contrast, when a Au NS is attached to the terrace of the NP, the coupling is weaker, as manifested by a smaller 3-nm red-shift. Simulations were performed as described in section 3.3. The NS was $27 \mathrm{~nm}$ in diameter. Simulated configurations and NS locations are shown for each case and are representative of the experimental structures. The surfaces of the NP and the NS were separated by a distance of $1 \mathrm{~nm}$, measured along the long-axis of the hexagon for the NS placed on the vertex and along the thickness direction for the NS placed on the terrace. The simulated shifts are somewhat lower than those in the experiment; it is possible that the actual surface-to-surface distance is lower than $1 \mathrm{~nm}$, the value used in the simulation model. After all, coupling-induced shifts increase rapidly with decreasing inter-particle distance. ${ }^{69,80,85}$ Nevertheless the simulated site-dependence of the shift is in qualitative agreement with the observations.

It is instructive to spatially resolve the site-dependence of plasmon coupling even further, for instance, by determining if there is a systematic difference in the magnitude of shift between specific locations on the terrace or between those on the sides of the NP. While this was difficult to perform in a statistically significant manner using the limited experimental cases, simulations proved useful in this regard (Figure 3.5 and Figure 3.6). 

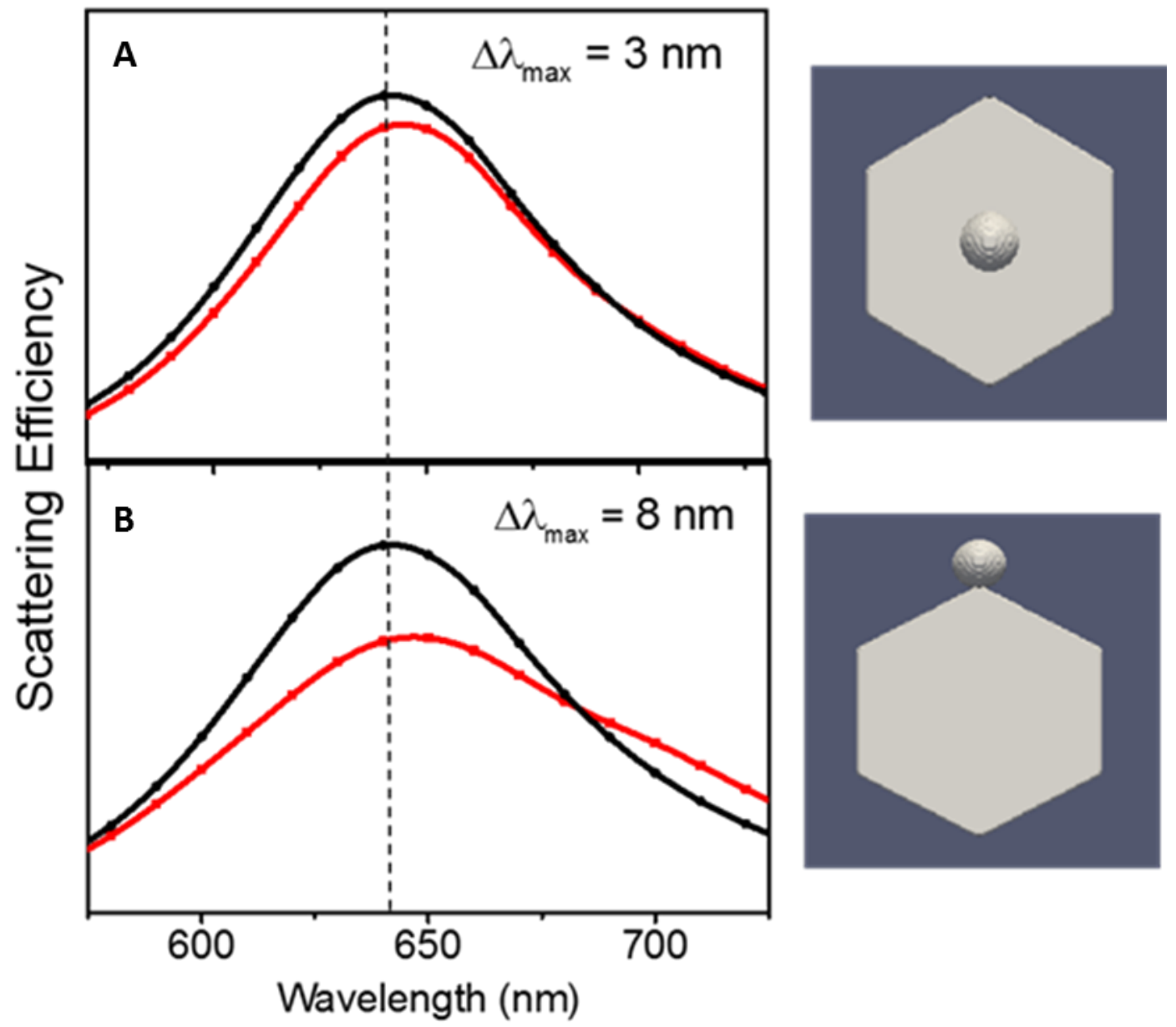

Figure 3.9 Terrace site vs. edge site attachment simulation results (A) Scattering spectrum of a single Au NP before (black line) and after (red line) attachment of a $27 \mathrm{~nm}$ diameter Au NS on an inner terrace site of the NP, as shown in the simulated configurations to the right. (B) Scattering spectrum of a single Au NP before (black line) and after (red line) attachment of a 27-nm diameter Au NS on a vertex site, as shown in the simulated configurations to the right. The $\lambda_{\max }$ values were determined by fitting the spectra to single Lorentzian peaks. The shift in $\lambda_{\max }$ upon NS attachment is indicated. 
The observed site-dependence originates from the spatio-polar variation of the polarizability of the NP, qualitatively analogous to the tensorial nature of the molecular polarizability of benzene. ${ }^{143}$ A simulation of the near-field around a resonantly excited NP (Figure 3.10) serves as a map of the polarizability of the NP. Simulations were performed for a hexagonal Au NP with a long axis of $137 \mathrm{~nm}$, a thickness of $31 \mathrm{~nm}$, and a $1 \mathrm{~nm}$ thick ligand shell of HT and 4-ATP, representative of the experimental structure. Under in-plane excitation, the NP is most polarizable at its vertices, followed by the edges. The NP is least polarizable at the central regions of terraces and side faces. Thus, a NS, with an induced dipole excited in the plane of the NP, is able to polarize a NP most strongly when it is attached along the sides in contact with the vertices of the NP. In addition to vertex attachment, there is an additional requirement for strong coupling: the excitation must be polarized along the NP/NS axis, such that the dipolar plasmon oscillation of the NP is aligned head-to-tail with the dipolar plasmon oscillation of the NS (Figure 3.4). These conditions lead to a strong attractive interaction, resulting in a large red-shift of the NP localized surface plasmon resonance (LSPR). ${ }^{80}$

On the other extreme, attachment of the NS at the terrace causes minimal polarization of the NP. While the side-by-side interaction does not allow for a strong interaction between the dipolar LSPR oscillations of the NP and the NS, there is an observable attractive coupling between the dipolar oscillation of the NS and an induced image dipole on the much larger NP surface, as seen from field maps shown in Figure 3.8. However, this interaction has only a weak effect on the NP dipolar LSPR, manifested as a small red-shift of the latter. ${ }^{80}$ Furthermore the nature of this interaction is such that the lateral position of the terrace-attached NS in relation to the NP edges is not too important in determining the magnitude of the coupling-induced shift (Figure 3.6). In summary, the 


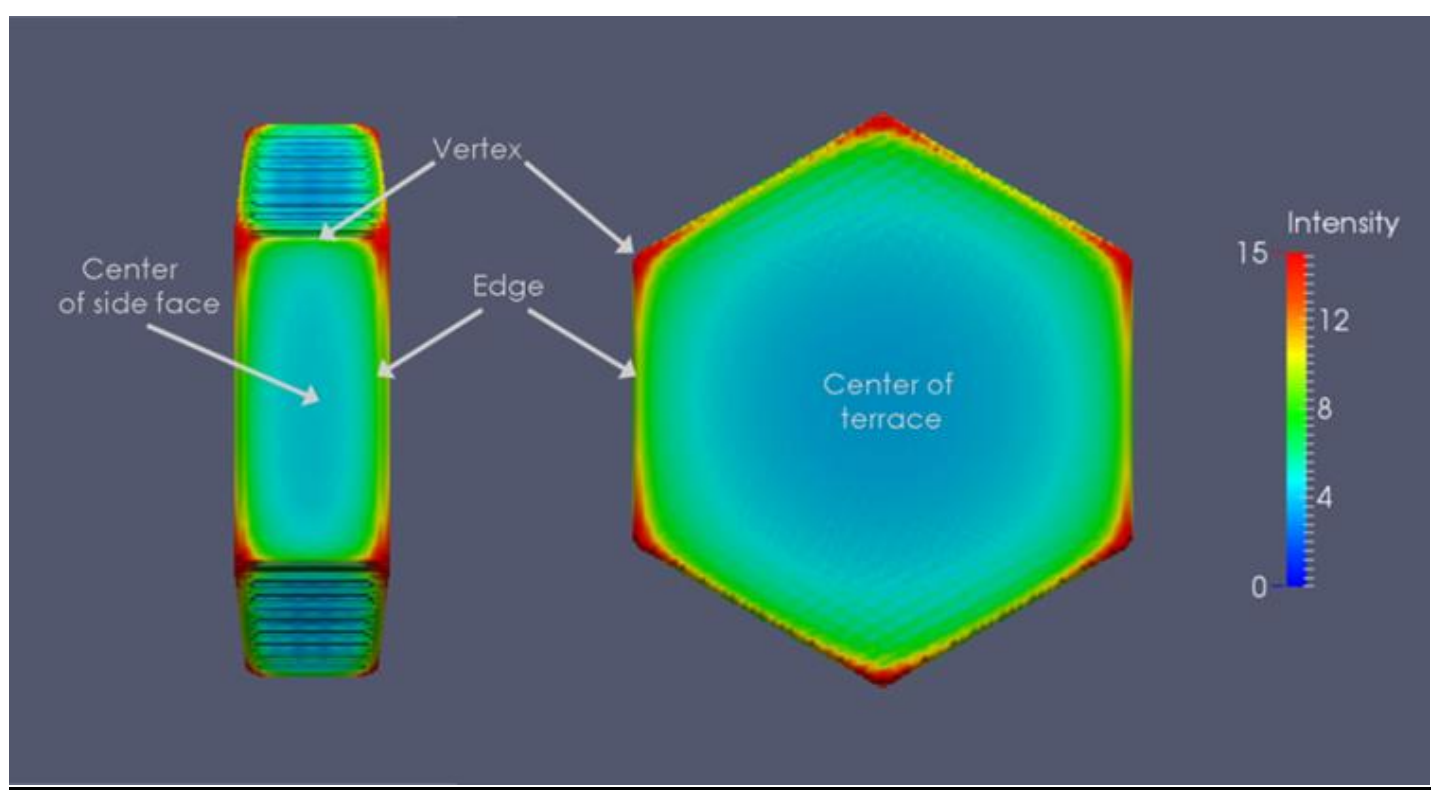

Figure 3.10 Spatial map of the NP polarizability. Simulated near-field over the surface of a $\mathrm{Au}$ NP excited by $645-\mathrm{nm}$ unpolarized light. The near-field is depicted in the form of an amplitude enhancement $\left(|\mathrm{E}| /\left|\mathrm{E}_{0}\right|\right)$ on a color scale. 
overall trend seen in the coupling-induced red-shift, i.e., vertices $>$ side faces $>$ terrace sites, is essentially a reflection of the spatial map of the NP polarizability under in-plane excitation. Experimentally, however, we were unable to resolve a difference in coupling strength for vertex vs. side face sites from the limited number of structures available (Table 2). This inability likely resulted from the heterogeneities in i) the exact position of the NS relative to a vertex (Figure 3.5), ii) the actual distance of the NS from the NP surface, iii) the degree of roundedness of the NP sides, and iv) the size of the NS, all of which influence the coupling-induced shift, apart from the attachment location of the NS.

\subsubsection{NP with Multiple NSs Attached}

NSs symmetrically distributed around a NP: Further studies with multiply attached NSs indicate a unique synergistic effect, whereby the polarizing effect of a NS on the NP is positively assisted by the presence of other attached NSs. As discussed earlier, the attachment of a single NS to the NP sides results in a $22 \mathrm{~nm}$ red-shift, on average. With three NSs attached to the sides (Figure 3.11A), a $69 \mathrm{~nm}$ red-shift is seen, amounting to a shift of $23 \mathrm{~nm}$ per attached NS. However, the attachment of four NSs around the NP sides is seen to result in a total red-shift of $122 \mathrm{~nm}$ (Figure 3.11B), which amounts to a shift of $31 \mathrm{~nm} / \mathrm{NS}$, considerably larger than the effect produced by a single NS. This observation is in contrast to the behavior observed in core-satellite assemblies, where a linear LSPR shift is observed with an increasing number of satellite NSs. ${ }^{94}$

While experimental heterogeneity makes it difficult to unequivocally confirm this synergistic effect, the accompanying simulations provide support for this conclusion. In simulations, the attachment of one NS to a vertex position results in a $8 \mathrm{~nm}$ shift, as 

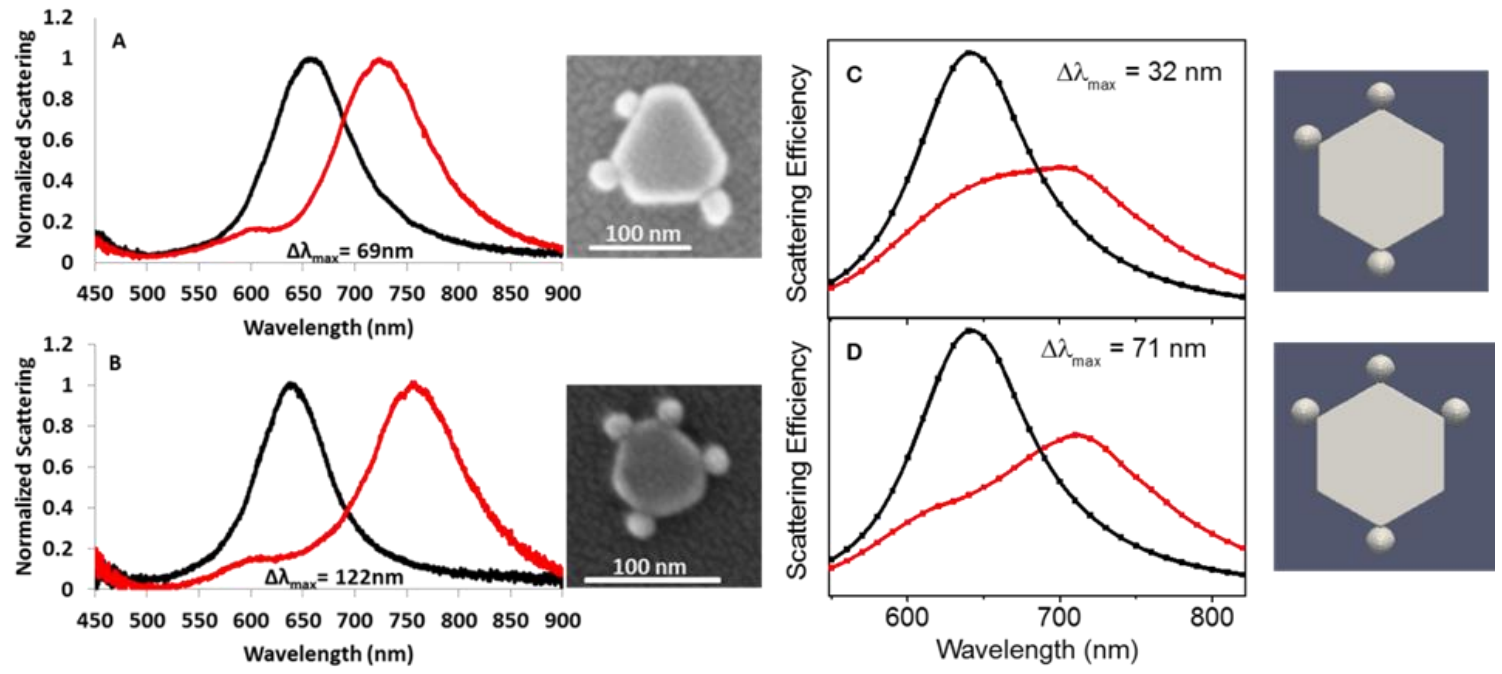

Figure 3.11 Experimental (A \& B) and simulated (C \& D) plasmonic coupling between a Au NP and multiple NSs. Dark-field scattering spectra of Au NPs before (black) and after (red) three (A) and four (B) $\sim 25 \mathrm{~nm}$ diameter Au NSs are attached to the sides of the Au NPs. The SEM images of the corresponding assemblies are shown on the right.Simulations were performed as described in section 3.3. Here, $27 \mathrm{~nm}$ diameter Au NSs were placed at three (C) or four (D) vertices along the sides of the NP (red data-points connected by cubic splines). Simulated configurations and NS locations are shown for each case and are representative of experimental structures. For attachment of three NSs, the LSPR wavelength maximum was determined by fitting the simulated scattering spectrum to a single Lorentzian peak. For four NSs, the spectrum was fit to two Lorentzian peaks. The red-shift $\Delta \lambda_{\max }$ indicated for each case is relative to the $\lambda_{\max }$ for a NP without an attached NS (spectrum shown by black data-points connected by a cubic spline). Unpolarized light was used for excitation in the simulations, similar to the conditions in experiment. 
discussed earlier (Figure 3.9). However, with three NSs attached to three vertices of the NP (Figure 3.11C), the red-shift is $32 \mathrm{~nm}$, amounting to a shift of ca. $11 \mathrm{~nm} / \mathrm{NS}$. Four NSs attached to four vertices of the NP (Figure 3.11D), give rise to an overall shift of 71 $\mathrm{nm}$ or ca. $18 \mathrm{~nm} / \mathrm{NS}$. The synergistic effect found here is analogous to the positive enhancement in the molecular polarizability of benzene by certain activating functional groups. ${ }^{144}$

NSs asymmetrically distributed around NP: In assemblies with multiple NSs, the respective placement of NSs on the NP must dictate the nature of plasmonic coupling and the resulting spectral characteristics of the assembly. In contrast to the uniform distribution of NSs, shown in Figure 3.11, we found some instances of NSs asymmetrically distributed around the NP sides (Figure 3.12A, B). These structures exhibited broad, asymmetric, and complex spectra, quite unlike those seen with the symmetric distributions. The strongest scattering band of these asymmetric structures appears to be much more strongly red-shifted than in the case of an equivalent (with the same number of NSs) symmetric distribution, e.g., $\Delta \lambda_{\max }=132 \mathrm{~nm}$ in Fig. 3.12A vs. 122 $\mathrm{nm}$ in Fig. 3.11B. DDA simulation of such a NP/NS assembly, excited with unpolarized light, reproduced the broad, asymmetric nature of the LSPR spectrum (Figure 3.12C) with the strongest scattering band red-shifted by as much as $79 \mathrm{~nm}$. This red-shift is larger than that for the symmetric distribution with four NSs in Figure 3.11D $\left(\Delta \lambda_{\max }=71\right.$ nm under unpolarized excitation).

The complex nature of the spectrum is a result of the large polarization anisotropy of the asymmetric NP/NS assemblies, as shown by further polarization-dependent simulations. Due to the asymmetry of the structures, the strength of coupling between the NP and NSs is strongly dependent on the polarization direction. The latter is manifested 

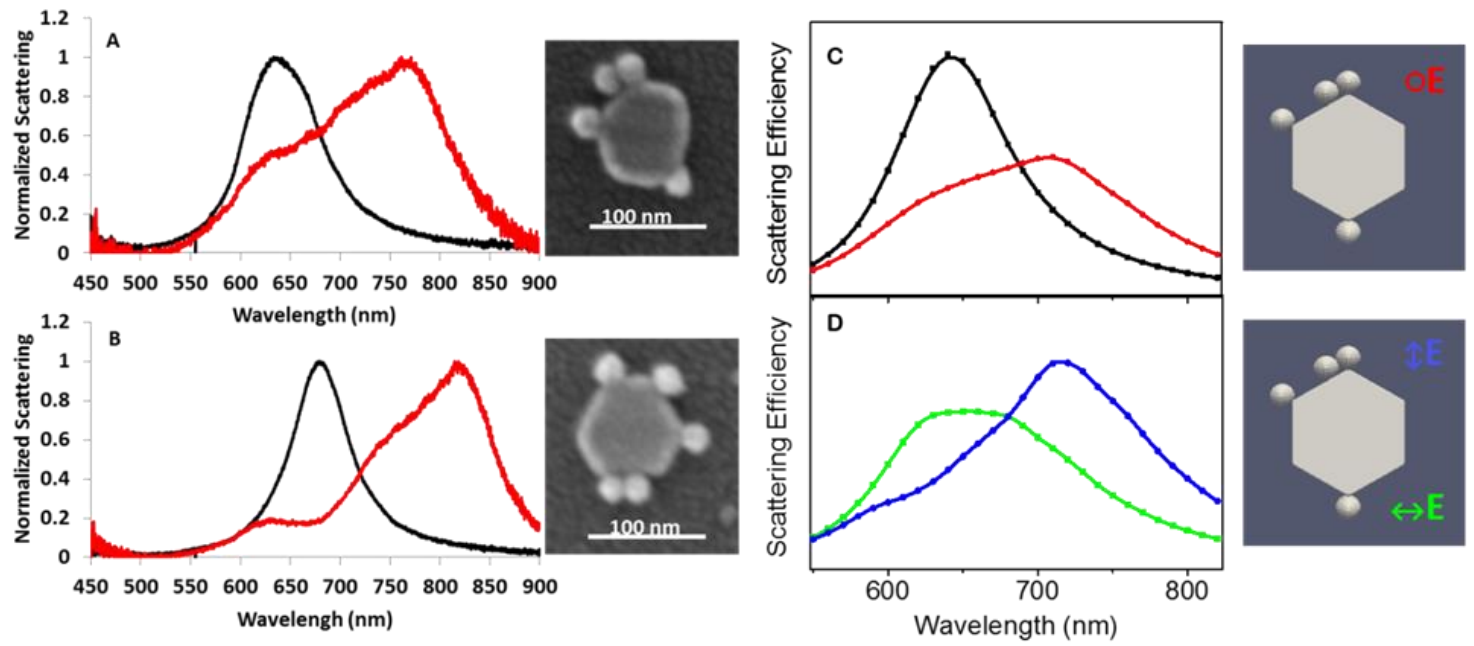

Figure 3.12 Experimental (A \& B) and simulated (C \& D) plasmonic coupling between a Au NP and multiple asymmetrically distributed NSs. Dark-field light-scattering spectra of a roughly hexagonal $\mathrm{Au} \mathrm{NP}$ before (black) and after (red) attachment of four (A) and five (B) asymmetrically distributed NSs to the sides of the NP. The SEM images of the corresponding assemblies are shown on the right. Simulations were performed as described in the section 3.3. Here, four 27-nm diameter Au NSs were placed along the sides of the NP. The simulated configuration, representative of the experimental configuration in (A), is shown besides each simulated spectrum. In (C), the simulated spectrum of the NP/NS assembly (red data-points connected by a cubic spline) under unpolarized light excitation is shown alongside the simulated spectrum of a NP with no NSs attached (black data-points connected by a cubic spline), also for unpolarized light excitation. In (D), simulated spectra of the NP/NS assembly are shown for light polarized along the NP short-axis (green data-points connected by a cubic spline) and for light polarized along the long-axis (blue data-points connected by cubic spline). The spectrum for long-axis polarization, fit to two Lorentzian peaks, shows a lower energy band centered at 721 $\mathrm{nm}$, red-shifted from the NP LSPR by as much as $79 \mathrm{~nm}$. The spectrum for short-axis polarization, fit to one Lorentzian peak, shows a band centered at $656 \mathrm{~nm}$. 
by the large disparity in the simulated scattering spectra for the two orthogonal polarization directions (Figure 3.12D). The symmetric distributions also show similar polarization anisotropy (Figure 3.14), but this effect is much starker for the asymmetric distributions. For the representative asymmetric structure simulated, three NSs are located along the long-axis direction, whereas only one NS is located in the short-axis direction. As a result, coupling along the long-axis direction is significantly stronger than that along the short-axis direction. The spectrum under long-axis polarization exhibits at least two modes, including a strong highly red-shifted mode centered at $721 \mathrm{~nm}$ and a weaker blue-shifted (as discussed later, a quadrupolar) mode around $613 \mathrm{~nm}$. On the other hand, the short axis-polarization results in a broad band centered around $656 \mathrm{~nm}$. With unpolarized light excitation, which samples all directions equally, the spectrum comprises of these multiple coupled modes corresponding to different polarization directions and therefore the spectrum is multi-peaked and asymmetric. This is unlike the case of symmetric distributions where modes polarized along the two orthogonal directions are closer to each other in energy and in intensity; therefore the composite spectrum is more symmetric and the composite red-shift is somewhat smaller. A similar effect is shown in Figure 3.13 for multiple NSs distributed on terrace sites of somewhat asymmetric NPs. When multiple NSs were distributed non-symmetrically on inner terrace sites and near terrace edges, we often observed broadening resulting from the appearance of a new peak red-shifted as much as $35-40 \mathrm{~nm}$ relative to the initial dipolar mode of the NP. This phenomenon is a result of increased symmetry breaking in the coupled NP/NS assemblies. This effect appeared to be starker when the initial NP itself was not very symmetric, as seen from the representative cases shown in this figure. 

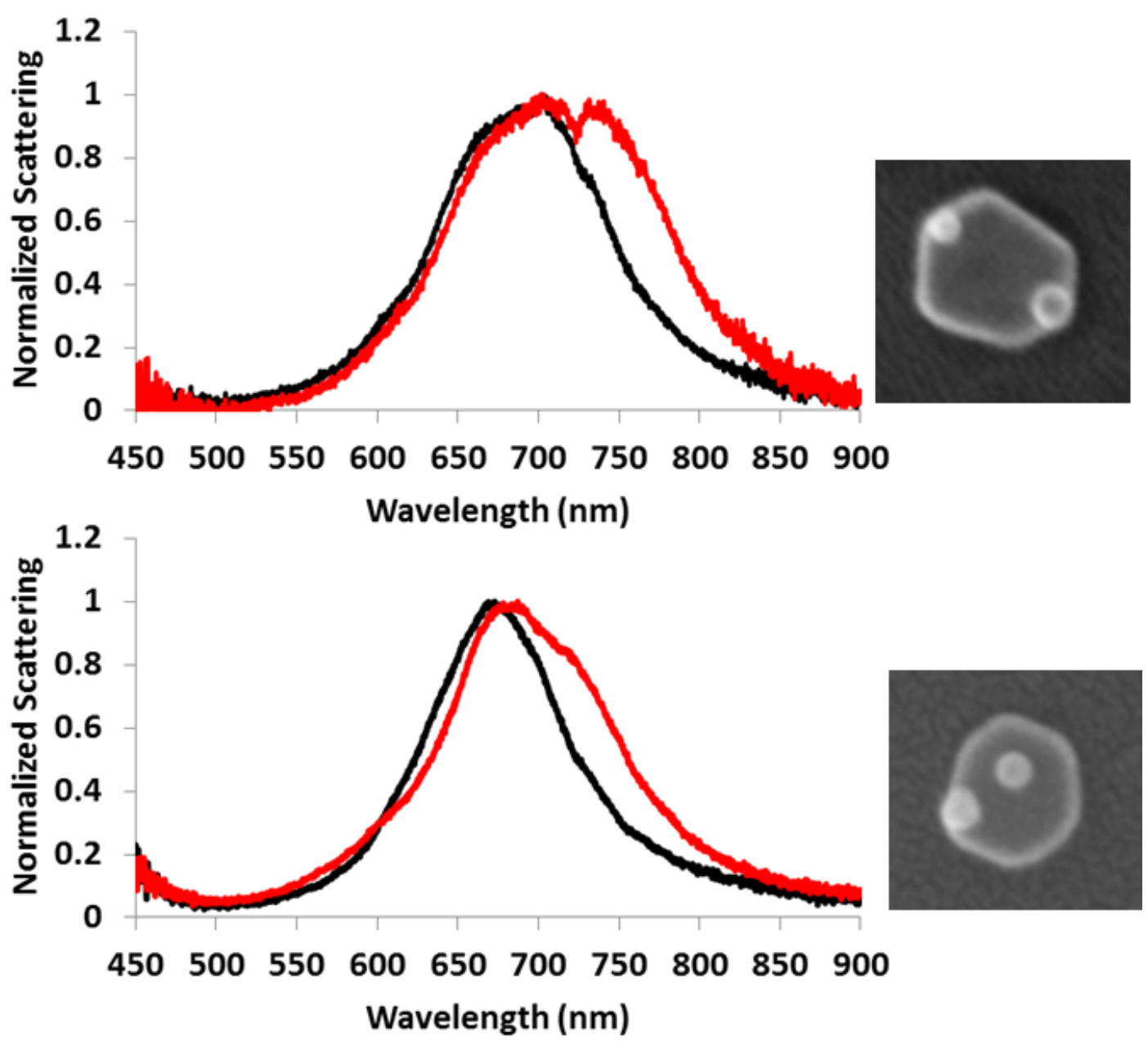

Figure 3.13 Single-particle dark-field light-scattering spectra of a roughly hexagonal Au NP before (black) and after (red) attachment of multiple NSs to NP terrace sites. Final morphologies are displayed on the right. 
An additional effect is seen with multiply attached NSs, both in experiment and in simulations (Figures 3.11 and 3.12). Strong coupling with multiple NSs attached on the NP sides induces a new mode around $625 \mathrm{~nm}$, blue of the dipolar LSPR of the NP (Figure 3.11A, C). Whereas a weak shoulder is seen with one NS attached (Figure 3.2B), with four NSs attached, this new mode is strong enough to be clearly resolved from the LSPR peak (Figure 3.11B, D). An electric-field simulation indicates that this mode is the result of a quadrupolar excitation of the NP/NS assembly polarized along the short in-plane axis of the NP (Figures 3.14 and 3.15).

Figure 3.14 shows that the redshift in the dipolar LSPR resulting from coupling is smaller when light is polarized parallel to the short axis of the hexagon as compared to the case when light is polarized parallel to the long-axis of the hexagon. However, under short-axis polarization, coupling results in the emergence of a prominent high energy mode centered at $613 \mathrm{~nm}$, in addition to the dipolar mode at $705 \mathrm{~nm}$. These $\lambda_{\max }$ values were determined by fitting the spectrum to two Lorentzian peaks. The scattering spectrum for long-axis polarization, fit to two Lorentzian peaks, yields a strong dipolar mode with a $\lambda_{\max }$ at $717 \mathrm{~nm}$ and only a weak shoulder in the blue region. In order to correctly assign those modes, a near-field was simulated in Figure 3.15.

When the incident light is polarized parallel to the long axis of the hexagon, the near-field appears to be concentrated most at the extremities of the NSs located along the polarization direction, as well as at the junctions between the NS and NP vertices. As indicated by its respective electrostatic charge distribution, the field plot represents a dipolar mode of coupling between the NP and the two NSs, which is manifested as the LSPR peak centered at $717 \mathrm{~nm}$ in Figure 3.14. A similar dipolar mode of excitation is seen when light with a wavelength of $705 \mathrm{~nm}$ is polarized along the short axis of the 
hexagon. However, when the short axis of the hexagon is excited using $613 \mathrm{~nm}$ wavelength light, corresponding to the higher energy peak in Figure 3.14, the field appears to be concentrated primarily at the junctions between the NS and NP vertex. There is lower field enhancement at the extremities of the NSs along the polarization direction. This field pattern is indicative of a higher-energy quadrupolar mode of excitation, as depicted by its respective electrostatic charge distribution.

\subsection{CONCLUSIONS}

In summary, we designed, in analogy with benzene-derived molecular structures, complex plasmonic nanoassemblies by regioselectively attaching Au NSs to specific sites on the surface of a large hexagonal Au NP. In these assemblies, we find that the site of NS attachment dictates the plasmonic response of the NP. NSs attached on the sides, especially at vertices, couple strongly with the NP, whereas NSs attached on the terraces cause little perturbation of the NP plasmon resonance. Such regioselective placement of satellite NSs on the surface of a NP has potential for producing unique structuredependent electromagnetic modes with interesting spectral attributes and near-field distributions. As one example of unique behavior, we find that the polarizing effect of multiple NSs on the NP is synergistic. As another example, we find that the optical response is dramatically different for an assembly with a uniform distribution of NSs around the NP versus one with an asymmetric distribution. The regioselectivity of plasmon coupling discussed here can be exploited for 3D spatial sensing with nanometer resolution. ${ }^{134}$ For instance, it may be possible to construct a device consisting of a NP linked to a NS via a protein of interest. The device may be designed such that the occurrence of a key conformational change in the protein results in the migration of the 


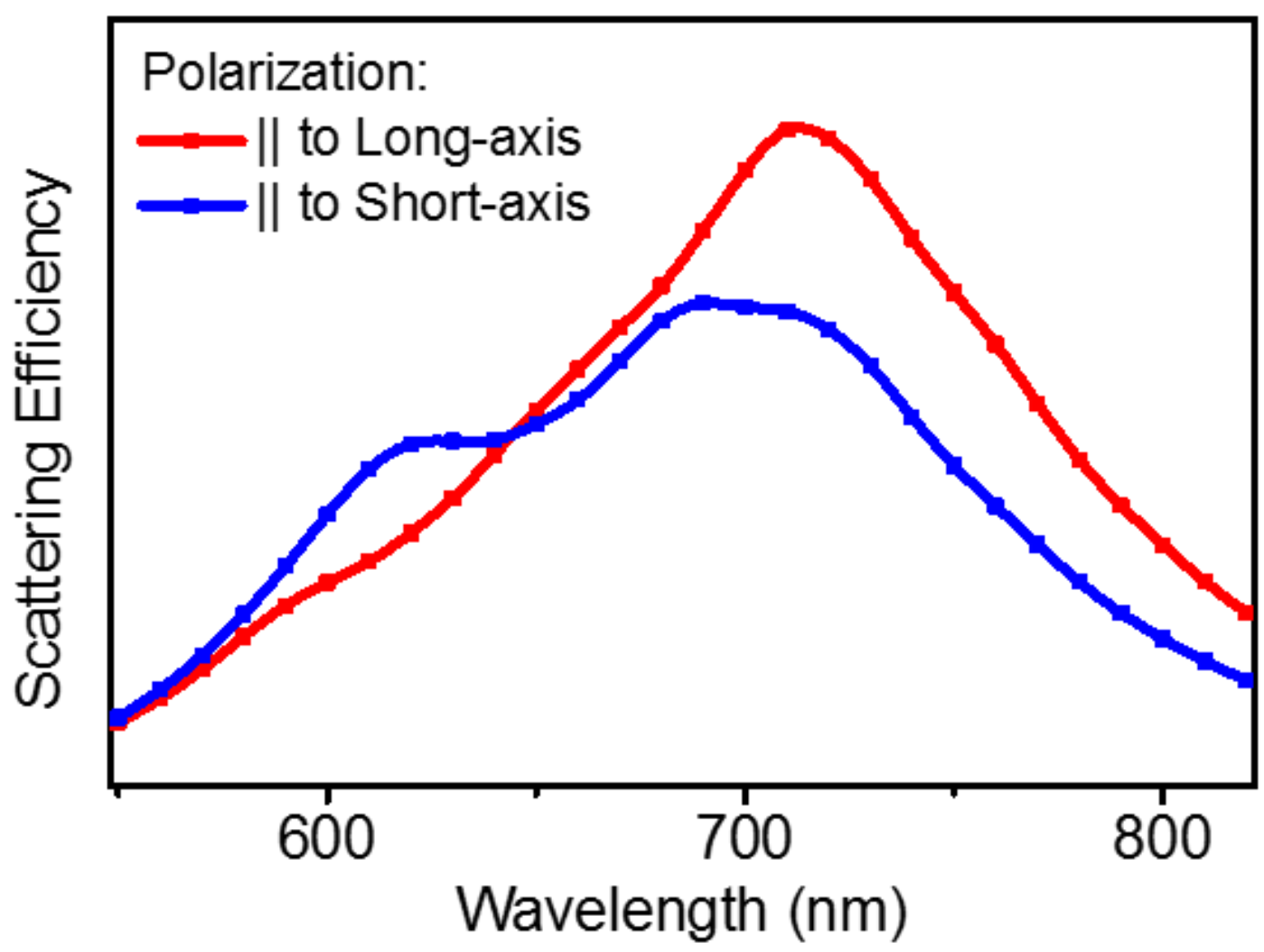

Figure 3.14 Simulated effect of the incident light polarization on the plasmon coupling between an Au NP and four NSs. Simulations were performed for a hexagonal Au NP with a long axis of $137 \mathrm{~nm}$, a thickness of $31 \mathrm{~nm}$, and a 1-nm thick ligand shell of HT and 4-ATP, representative of the experimental structure. Four Au NSs, each with a diameter of $27 \mathrm{~nm}$, were attached to four of the vertices of the NP, (configuration is shown in Figure 3.16). The surface-to-surface distance between the NP and each NS was $1 \mathrm{~nm}$, measured along the long-axis of the hexagon. 

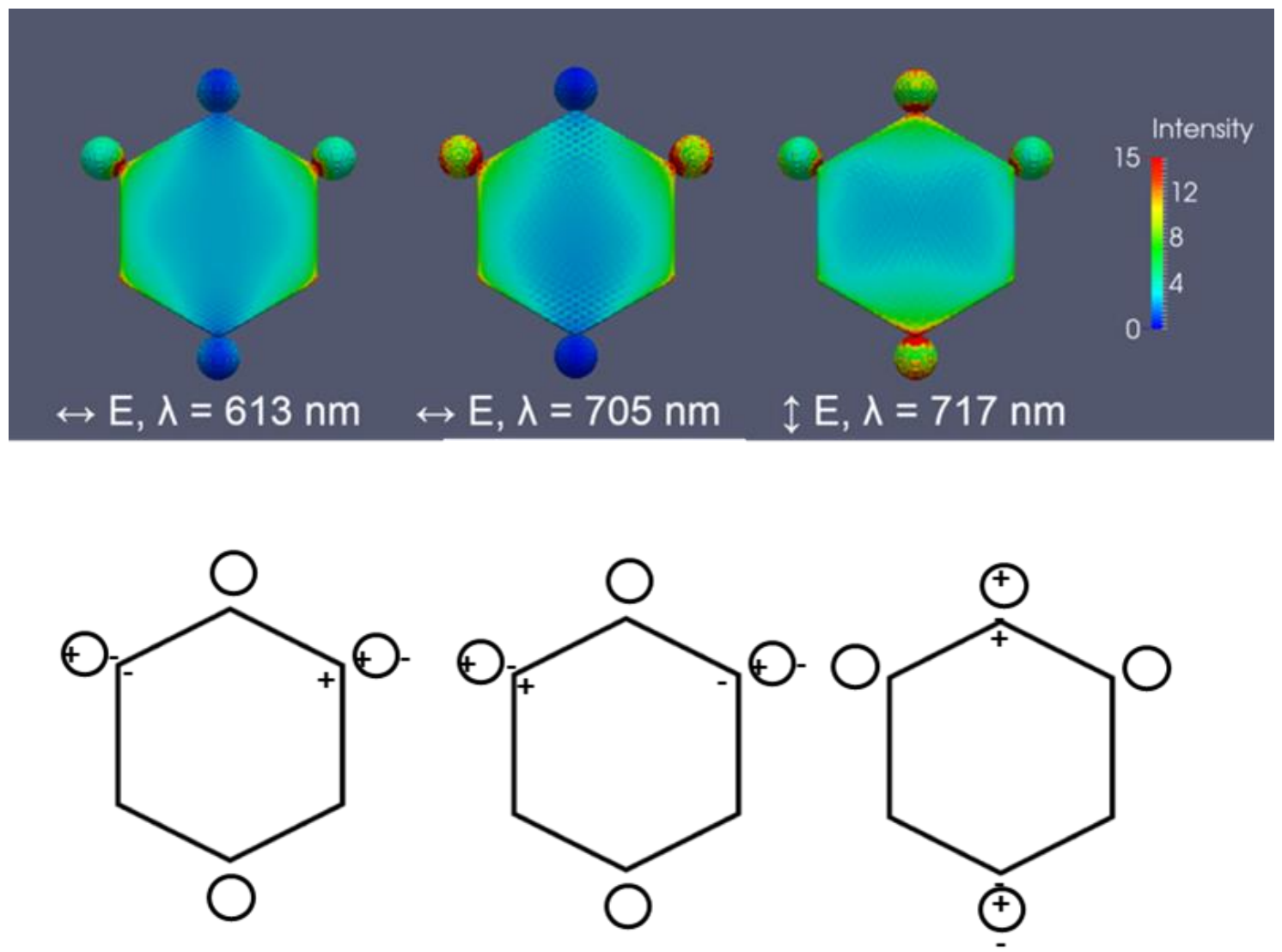

Figure 3.15 Simulated near-field over the surface of a Au NP coupled to four Au NSs. The nearfield is depicted in the form of an amplitude enhancement $\left(|\mathrm{E}| /\left|\mathrm{E}_{0}\right|\right)$ on a color scale. Simulations were performed for a hexagonal Au NP with a long axis of $137 \mathrm{~nm}$, a thickness of $31 \mathrm{~nm}$, and a 1-nm thick ligand shell of HT and 4-ATP, representative of the experimental structure. Four Au NSs, each with a diameter of $27 \mathrm{~nm}$, were attached to four of the vertices of the NP, as depicted. The surface-to-surface distance between the NP and each NS was $1 \mathrm{~nm}$, measured along the longaxis of the hexagon. Three different simulations were run: the direction of polarization and wavelength of incident light employed in each simulation are noted for each near-field plot. An electrostatic charge distribution corresponding to each near-field plot is also shown below each plot. 
attached NS from the sides of the NP to the outer terrace region. Our results show that the spectral shift resulting from such a dynamic configuration change would be optically detectable on the level of single nanodevices. Future work will be geared toward the achievement of increasingly finer and more dynamic regioselective control and the exploitation of such control in functional plasmonic devices. 


\section{CHAPTER IV}

\section{THE EFFECT OF NANOSPHERE SIZE ON THE PLASMONIC PROPERTIES OF REGIOSELECTIVELY-COUPLED GOLD NANOPLATE-NANOSPHERE STRUCTURES AT THE SINGLE PARTICLE LEVEL}

In this chapter, we describe the dark-field light scattering properties of size- and shape-mismatched heterodimer nanostructures consisting of a $\mathrm{Au}$ nanosphere (NS) coupled to a Au nanoplate (NP). In these heterodimers, the shape of the NP was roughly hexagonal or a truncated triangular shape, while the NS was spherical in shape and variable size $(13 \mathrm{~nm}, 24 \mathrm{~nm}$, or $51 \mathrm{~nm}$ in average diameter). The location where the NS attached to the NP varied between the side of the plate (edge or vertex) and the top terrace while the spacing between them was constant, dictated by the length of the molecular linker, 4-aminothiophenol (4-ATP). Strong dipolar coupling occurred for all NP/NS heterodimers when the NS was located on the side of the plate as opposed to very weak coupling for those located on the top terrace. The positive shift in the most redshifted dipolar plasmon mode of the NP/NS heterodimer relative to the original NP increased with increasing NS size for those attached on the side of the NP in the order of $9 \pm 2 \mathrm{~nm}, 24 \pm 4 \mathrm{~nm}$, and $98 \pm 16 \mathrm{~nm}$ for the 13,24 , and $51 \mathrm{~nm}$ average diameter NSs, respectively. This mode is mainly due to polarization parallel to the NP/NS long axis. As the NS size increased, this peak red-shifted enough to easily observe two other plasmon modes in the spectrum, one of them is a red-shift and due to polarization 
perpendicular to the long NP/NS axis and the other is a blue-shifted antibonding type mode. With smaller NSs, the shift due to light polarized parallel to the coupling axis is not large enough to distinguish it from that due to light polarized perpendicular to the coupling axis, making those two peaks overlap with an overall broader peak.

For NSs attached to the top terrace of the NPs, the shift in the dipolar plasmon mode was $1 \pm 1 \mathrm{~nm}, 3 \pm 1 \mathrm{~nm}$, and $14 \pm 4 \mathrm{~nm}$ for the 13,24 , and $51 \mathrm{~nm}$ diameter NSs, respectively. This clearly shows much weaker coupling compared to the structures with NSs attached to the edge, due to the dipolar mode of the NP and NS being aligned parallel to one another. The spectrum did not show a blue-shifted multipolar mode or separate peaks due to different symmetry along different polarizations directions for any of the heterodimers since the shifts were relatively small.

In addition to the dimer structures, we present results on multiple NSs coupled to edge and terrace sites of NPs. In general, this increases the shift in the modes and increases the complexity of the spectra due to lower symmetry in the structure. This work provides new quantitative information about plasmon coupling between NPs and NSs as a function of size and location at the single nanostructure level, which is important for plasmonics and sensing applications. 


\subsection{INTRODUCTION}

Most of the coupled heterodimers described previously were composed of nanoparticles with fixed dimensions. There have only been a few reports about heterodimers consisting of nanoparticles with varied dimensions as described in this work. For example, Halas and co-workers described theoretically and experimentally size-asymmetric Au heterodimers of one size-fixed NS and one size-varied NS. ${ }^{1}$ The size-fixed NS had a diameter of $76 \mathrm{~nm}$, while the size of the other NS varied from $24 \mathrm{~nm}$ to $76 \mathrm{~nm}$. The dimer designed in this way allowed them to systematically study how the optical properties of the heterodimer depend on the size of the two NSs. However, there was no quantitative analysis of the relationship between LSPR shift and NS size. The Alivisatos Group studied Ag dimers of one $40 \mathrm{~nm} \mathrm{Ag} \mathrm{NS}$ and one Ag NS with two sizes, $20 \mathrm{~nm}$ and $40 \mathrm{~nm}$. They found that the spectrum was a linear combination of the longitudinal mode and transverse mode with symmetric dimers. When it came to asymmetric dimers, it turned out that the spectrum is not solely a linear combination of the two orthogonal modes. In their work, the authors also only focused on the plasmon modes rather than quantitating the LSPR shift caused by the different size NS. More importantly, the synthesis methods of heterodimers in most of the studies do not allow the spectrum of individual component particles to be measured since the dimers were synthesized in solution first and then drop casted to glass surface for spectra collection. Therefore, a simulation spectrum usually was provided for comparison between LSPR before and after coupling.

In this chapter, we describe the preparation of shape- and size- asymmetric NP/NS coupled nanostructures as in our previous study with varied NS size. ${ }^{21,145}$ Our strategy to assemble heterodimers allows us to obtain the LSPR spectrum of individual 
NPs before and after coupling with NSs. In the area of heterodimer plasmonic coupling, our research makes an important advancement by studying in detail the effect of NS size on the shape-asymmetric plasmonic coupling of NPs and NSs. Specifically, we quantify the magnitude of the plasmon shift upon coupling and qualitatively describe the observed plasmon modes for single and multiple NSs attached to different locations of the NP. This work provides insight and quantitative information about the effect of NS size on the site-selective coupling in geometrically complex Au nanostructures, such as Au NP/NS dimers.

\subsection{EXPERIMENTAL}

The synthesis of the Au NPs and Au NSs, preparation of nanostructure assemblies via various ligand-exchange reactions and characterization by LSPR and SEM are described in Chapter II.

\subsection{RESULTS AND DISCUSSION}

\subsubsection{Size-Dependent Plasmon Properties of NP/NS Dimers with NSs Attached to the Edge of NPs}

Using the procedure described in Chapter 2 and 3, we selectively bound one NS to the side edge of Au NPs that were $\sim 110 \mathrm{~nm}$ wide and $\sim 35 \mathrm{~nm}$ in height and measured the dark-field light scattering spectrum before and after NS attachment. Scanning electron microscopy (SEM) imaging showed the exact dimer structure which we correlated with the light scattering spectrum. We measured the light scattering and SEM 
images of various dimers with relatively constant NP size but varied NS size with diameters of $13 \pm 2 \mathrm{~nm}, 24 \pm 4 \mathrm{~nm}$, and $51 \pm 10 \mathrm{~nm}$. Figure 4.1 shows the single-particle dark-field spectra of a Au NP before and after attachment of a Au NS with the 3 different diameters. All of the NPs considered were hexagonal, truncated triangular, or circularshaped. Figure 4.1A shows a truncated triangular NP with an initial $\lambda_{\max }$ of $643 \mathrm{~nm}$ before NS attachment, which has three peaks after attachment of a $68 \mathrm{~nm}$ Au NS to the edge. All $\lambda_{\max }$ values were determined by single or multiple Lorentzian fitting. Comparing the spectra before attachment, two peaks red-shifted and one blue shifted. The most red-shifted peak is at $754 \mathrm{~nm}$, which positive shifted $111 \mathrm{~nm}$ from $643 \mathrm{~nm}$. Another red-shifted peak is around $670 \mathrm{~nm}$, which red shifted $27 \mathrm{~nm}$ from its original positon. A blue-shifted shoulder peak is around $600 \mathrm{~nm}$.

According to the simulation data in Figure 4.2, we can assign the peaks at $754 \mathrm{~nm}$, $670 \mathrm{~nm}$, and $\sim 600 \mathrm{~nm}$ to different LSPR modes. When the light polarization direction is parallel to the long interparticle axis of the NP/NS dimer, there are two modes in the LSPR spectrum (Blue line in Figure 4.2 A), a very intense red-shifted peak around 750 $\mathrm{nm}$ and a less intense blue-shifted peak around $600 \mathrm{~nm}$. The mode around $750 \mathrm{~nm}$ can be assigned to head-to-tail dipolar coupling of the NP and NS along the interparticle axis, analogous to $\sigma$ orbital formation. Head-to-tail dipole coupling is very strong (Figure 4.2 B) and results in a big red shift in the LSPR band. On the contrary, the mode around 600 $\mathrm{nm}$ can be attributed to head-to-head or tail-to-tail dipolar coupling of the NP and NS parallel to the interparticle axis, similar to $\sigma^{*}$ hybridization. Dipole coupling in this configuration is usually weak due to the cancellation of the dipole moments and results in a less intense peak (4.2 B). When light is polarized perpendicular to the interparticle axis, there is only one peak round $650 \mathrm{~nm}$ in the LSPR spectrum (Red line in Figure 4.2A), 

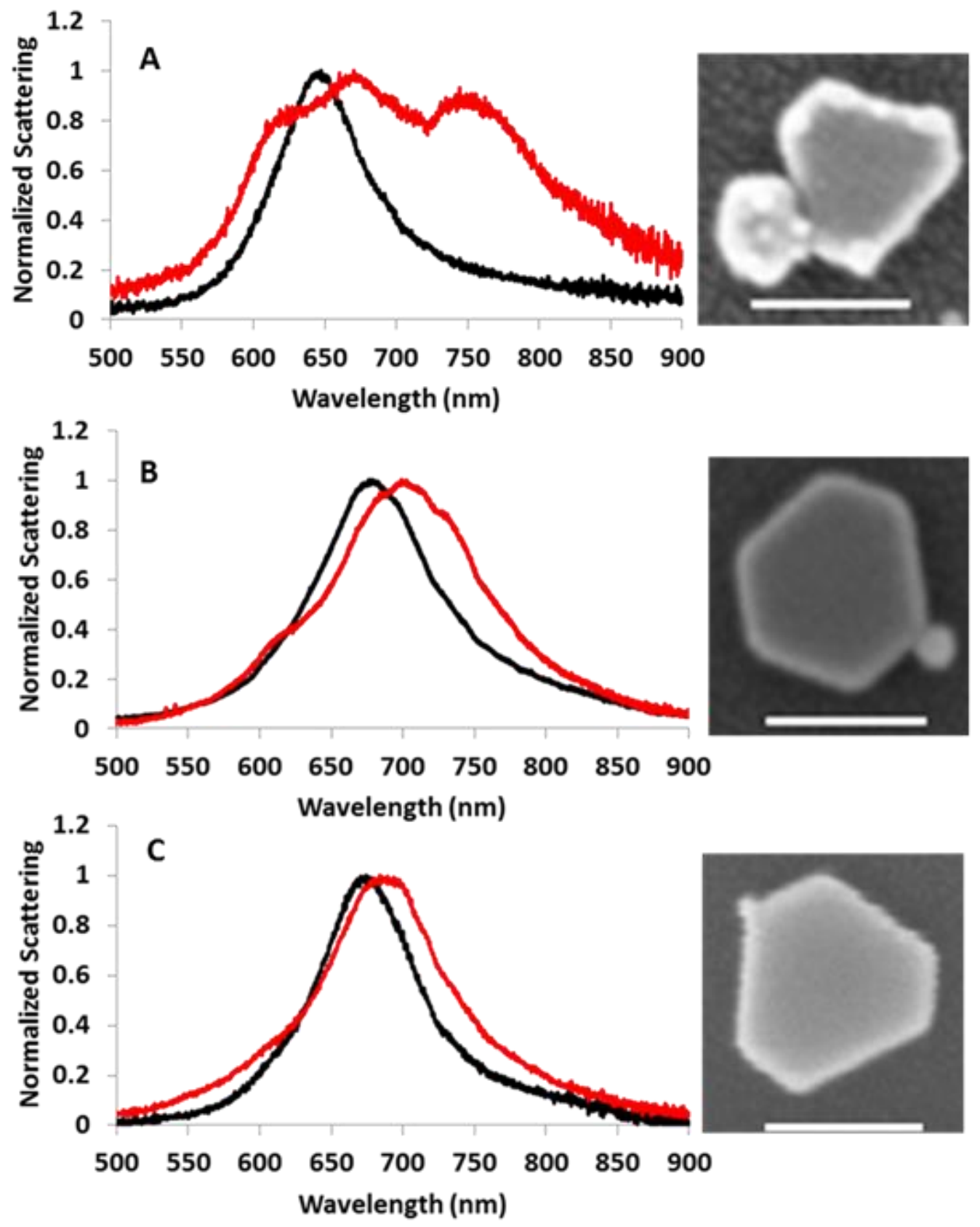

Figure 4.1. Dark-field scattering spectra of single NP before (black) and after (red) NS attached on the edge (left) and their SEM images (right). Scale bars in SEM images are $100 \mathrm{~nm}$. 

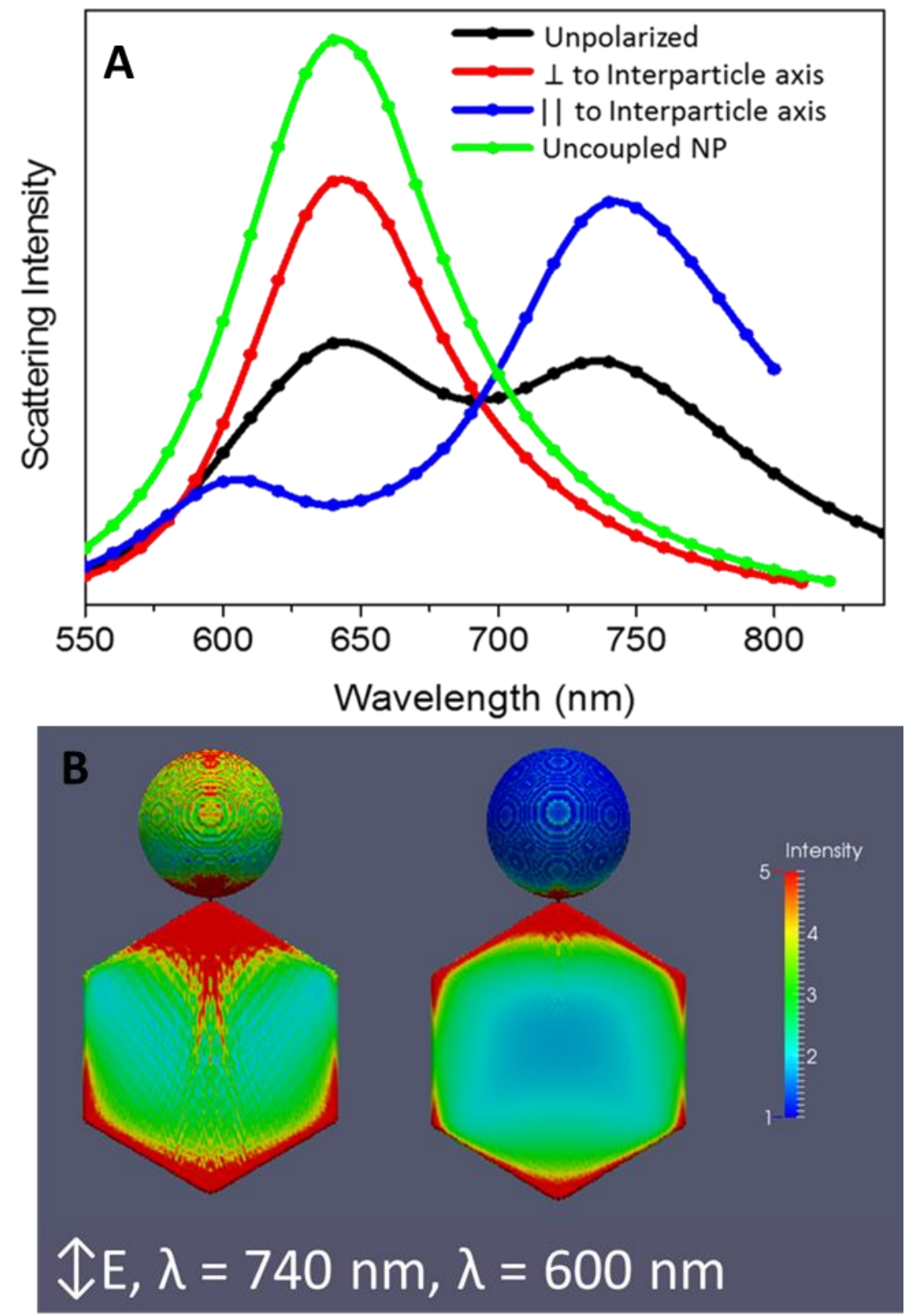

Figure 4.2. (A) Simulated scattering spectra for a hexagon NP coupled with a single NS with 67 $\mathrm{nm}$ in diameter under different polarization conditions. (B) The field enhancement at the surface for the nanoassembly, excited with light polarized along the interparticle axis at two different excitation wavelengths. 
which is similar to the LSPR spectrum of the NP before coupling (Green line in Figure 4.2A). This phenomenon tells us that the side-by-side dipolar coupling ( $\pi$ bond formation) is very weak and is manifested only as a very small red shift. Comparing the simulation data with our experimental results, we can assign the peak at $754 \mathrm{~nm}$ to headto-tail dipolar coupling of the NP and NS along the interparticle axis. We attribute the shoulder peak around $600 \mathrm{~nm}$ to $\sigma^{*}$ type dipolar coupling of the NP and NS. We assign the LSPR band around $670 \mathrm{~nm}$ to dipolar coupling of the NP and NS with light polarized perpendicular to the interparticle axis, analogous to $\pi$ orbital formation.

Figure 4.1B shows the result of coupling a relatively smaller NS with a diameter of $27 \mathrm{~nm}$ to a Au NP. There are two peaks in the LSPR spectra of the coupled NP/NS assembly. A red-shifted peak at $702 \mathrm{~nm}$ and a shoulder peak at $600 \mathrm{~nm}$. Similar to the data in Figure 4.1A, the NS and NP exhibit head-to-tail dipolar coupling causing a $22 \mathrm{~nm}$ red-shift from 680 to $702 \mathrm{~nm}$. This is significantly smaller compared to the red shift for the $68 \mathrm{~nm}$ NS. Also, there is a small shoulder peak at $600 \mathrm{~nm}$ whose intensity is much smaller compared to this mode for the $68 \mathrm{~nm}$ diameter NS. A separate third peak is not observed in this case because the dipolar plasmon coupling peak is not red-shifted enough to distinguish the third peak. Instead of observing this peak as two separate distinguishable peaks, it appears as one fairly broad peak. When the NS size decreases to $15 \mathrm{~nm}$ in Figure 4.1C, the dipolar red shift in $\lambda_{\max }$ is only $12 \mathrm{~nm}$. The blue shifted peak near $600 \mathrm{~nm}$ is now too weak to appear and the peak for light polarized perpendicular to the NP/NS axis is too close to the peak for light polarized parallel to the NP/NS axis, so they appear as one peak together, similar to Figure 4.1B. Table 4.1 shows the result of all of the NP/NS coupled structures as a function of NS size. 


\begin{tabular}{|c|c|c|c|c|}
\hline Initial $\lambda_{\max }(\mathrm{nm})$ & Final $\lambda_{\max }(\mathrm{nm})$ & Shift (nm) & NS Size (nm) & Morphology \\
\hline \multicolumn{5}{|c|}{ NSs with size average $=51 \pm 10 \mathrm{~nm}$} \\
\hline 636 & 749 & 113 & 56 & \\
\hline 635 & 738 & 103 & 44 & \\
\hline 651 & 750 & 99 & 46 & \\
\hline 643 & 754 & 111 & 68 & \\
\hline 653 & 743 & 90 & 52 & \\
\hline 669 & 740 & 71 & 41 & \\
\hline \multicolumn{5}{|c|}{ NSs with size average $=24 \pm 4 \mathrm{~nm}$} \\
\hline 653 & 667 & 14 & 22 & \\
\hline 673 & 696 & 23 & 20 & \\
\hline 673 & 702 & 29 & 28 & \\
\hline 678 & 699 & 21 & 27 & \\
\hline \multicolumn{5}{|c|}{ NSs with size average $=13 \pm 2 \mathrm{~nm}$} \\
\hline 681 & 689 & 8 & 13 & \\
\hline 665 & 672 & 7 & 12 & \\
\hline 691 & 700 & 9 & 15 & \\
\hline 669 & 680 & 11 & 12 & \\
\hline 675 & 687 & 12 & 15 & \\
\hline 680 & 690 & 10 & 13 & \\
\hline 667 & 675 & 8 & 14 & \\
\hline
\end{tabular}

Table 4.1. LSPRs and SEM images of representative single Au NPs with different size NSs attached on the edge sites of the NPs. 


\subsubsection{Size-Dependent Plasmon Properties of NP/NS Structures with NSs Coupled to the Terraces of NPs}

NSs of various sizes were controllably attached to the top terrace of the NPs. The simulation of the near-field around a resonantly excited NP shows the polarizability of the NP. ${ }^{146}$ Under in-plane excitation, a polarization map shows that the NP is most polarizable at its vertices, followed by the edges, and least polarizable at the terraces. When a NS attaches to the vertices or edges of the NP, its induced dipole excited in the plane of the NP can strongly polarize the NP. If the excitation is polarized along the NP/NS axis, the dipolar plasmon oscillation of the NP and NS is aligned head-to-tail, which leads to a large red-shift of the NP LSPR as shown in Figure 4.1. On the other hand, if the NS is attached to the terrace of the NP, the polarization of the two nanostructures is now aligned side-by-side and there is very weak coupling. This results in a negligible or very small red-shift of the NP LSPR compared to the head-to-tail interaction when the NS is attached to the side edge/vertex. For example, a single NP with an initial $\lambda_{\max }$ of $675 \mathrm{~nm}$ showed a modest red-shift of $12 \mathrm{~nm}$ upon attachment of a $43 \mathrm{~nm} \mathrm{Au} \mathrm{NS}$ at a terrace site on the NP (Figure 4.3A). Beside the red-shift, the attachment of a bigger size of NS on the terrace of the NP reduced the symmetry of the dipolar peak (red line in Figure 4.3A). When the size of the NS decreased to $22 \mathrm{~nm}$ as shown in Figure 4.3B, the amount of red-shift was only $3 \mathrm{~nm}$, which was almost the same level of shift caused by the local refractive index change of the monolayer attached to the NPs. Thus, the Au NP has only weak, if any, plasmon coupling with a $22 \mathrm{~nm}$ NS

attached to the terrace. The attachment of a much smaller $10 \mathrm{~nm}$ NS showed a similar amount of red-shift. In Figure 4.3C, the NP has a $\lambda_{\max }$ of $667 \mathrm{~nm}$ before attachment, which only shifts $1 \mathrm{~nm}$ upon attachment of a $10 \mathrm{~nm}$ NS on its terrace, which is negligible. 

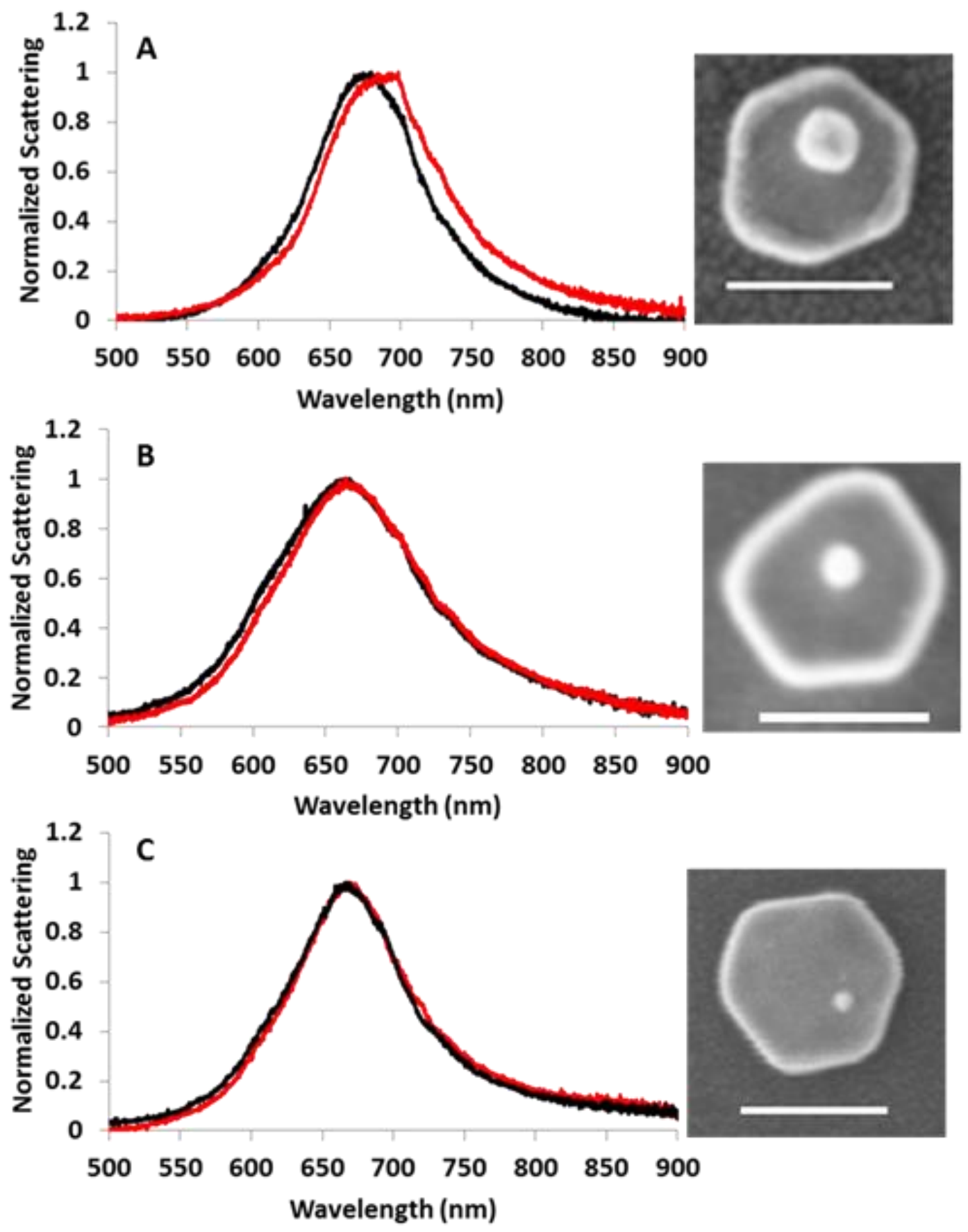

Figure 4.3. Dark-field light scattering spectra of a single NP before (black) and after (red) NS attached on the terrace (left) and their SEM images (right). Scale bars in the SEM images are 100 nm. 
Table 4.2 shows the data for all of the nanostructures studied as a function of NS size for those attached to the terraces.

To investigate the relationship between the dipolar plasmon shift and NS size, we plotted the shift as a function of the different NS size for attachment to edges and terraces as shown in Figure 4.4, which shows three trends. First, the magnitude of red shift increased with an increase in NS size regardless of the attachment location. The reason is the electric field decay is less dramatic as a function of distance for a larger sized NS. This means that the electric field will be stronger at some constant distance from the NS for a larger sphere. The magnitude of the electric field as a function of distance $\mathrm{S}$ for a NS of radius $\mathrm{R}$ is proportional to $[\mathrm{R} /(\mathrm{R}+\mathrm{S})]^{3}$. This means it increases at a constant distance $\mathrm{S}$ as a function of $\mathrm{R}$, but reaches a saturation value as $\mathrm{R}$ becomes very large relative to $\mathrm{S}$. The data follows this very simple model nicely, showing an increase in the shift for NSs of diameter from $10 \mathrm{~nm}$ to about $40 \mathrm{~nm}$ and saturate as it increases above 40 nm. NSs of diameter 40 to $60 \mathrm{~nm}$ show a very similar shift in $\lambda_{\max }$.

As already discussed, NSs attached to the edges of NPs result in a much larger red shift due to the stronger site-dependent coupling. The red shift of the dipolar plasmon mode for NSs attached to the edge was $\sim 10$ times larger than for those attached to a terrace. Specifically, the average shift was $98 \mathrm{~nm}$ for $51 \mathrm{~nm}$ diameter NSs attached to the edge and $11 \mathrm{~nm}$ for $42 \mathrm{~nm}$ NSs attached to the terrace. The average shift was $24 \mathrm{~nm}$ for $24 \mathrm{~nm}$ diameter NSs attached to the edge and $3 \mathrm{~nm}$ for $25 \mathrm{~nm}$ NSs attached to the terrace. The average shift was $9 \mathrm{~nm}$ for $13 \mathrm{~nm}$ diameter NSs attached to the edge and $1 \mathrm{~nm}$ for 13 nm NSs attached to the terrace. 


\begin{tabular}{|c|c|c|c|c|}
\hline Initial $\lambda_{\max }(\mathrm{nm})$ & Final $\lambda_{\max }(\mathrm{nm})$ & Shift (nm) & NS Size $(\mathrm{nm})$ & Morphology \\
\hline \multicolumn{5}{|c|}{ NSs with size average $=42 \pm 1 \mathrm{~nm}$} \\
\hline 675 & 687 & 12 & 43 & ○) \\
\hline 678 & 688 & 10 & 41 & (0) \\
\hline \multicolumn{5}{|c|}{ NSs with size average $=25 \pm 2 \mathrm{~nm}$} \\
\hline 657 & 659 & 2 & 25 & \\
\hline 659 & 661 & 2 & 26 & \\
\hline 660 & 664 & 4 & 24 & \\
\hline 679 & 682 & 3 & 23 & \\
\hline 686 & 688 & 2 & 29 & \\
\hline 707 & 709 & 2 & 25 & \\
\hline \multicolumn{5}{|c|}{ NSs with size average $=13 \pm 2 \mathrm{~nm}$} \\
\hline 667 & 668 & 1 & 10 & \\
\hline 693 & 694 & 1 & 15 & \\
\hline 674 & 676 & 2 & 12 & \\
\hline 683 & 684 & 1 & 13 & \\
\hline 676 & 678 & 2 & 14 & \\
\hline
\end{tabular}

Table 4.2. LSPRs and SEM images of representative single Au NPs with different size NSs attached on the terrace sites of the NPs. 


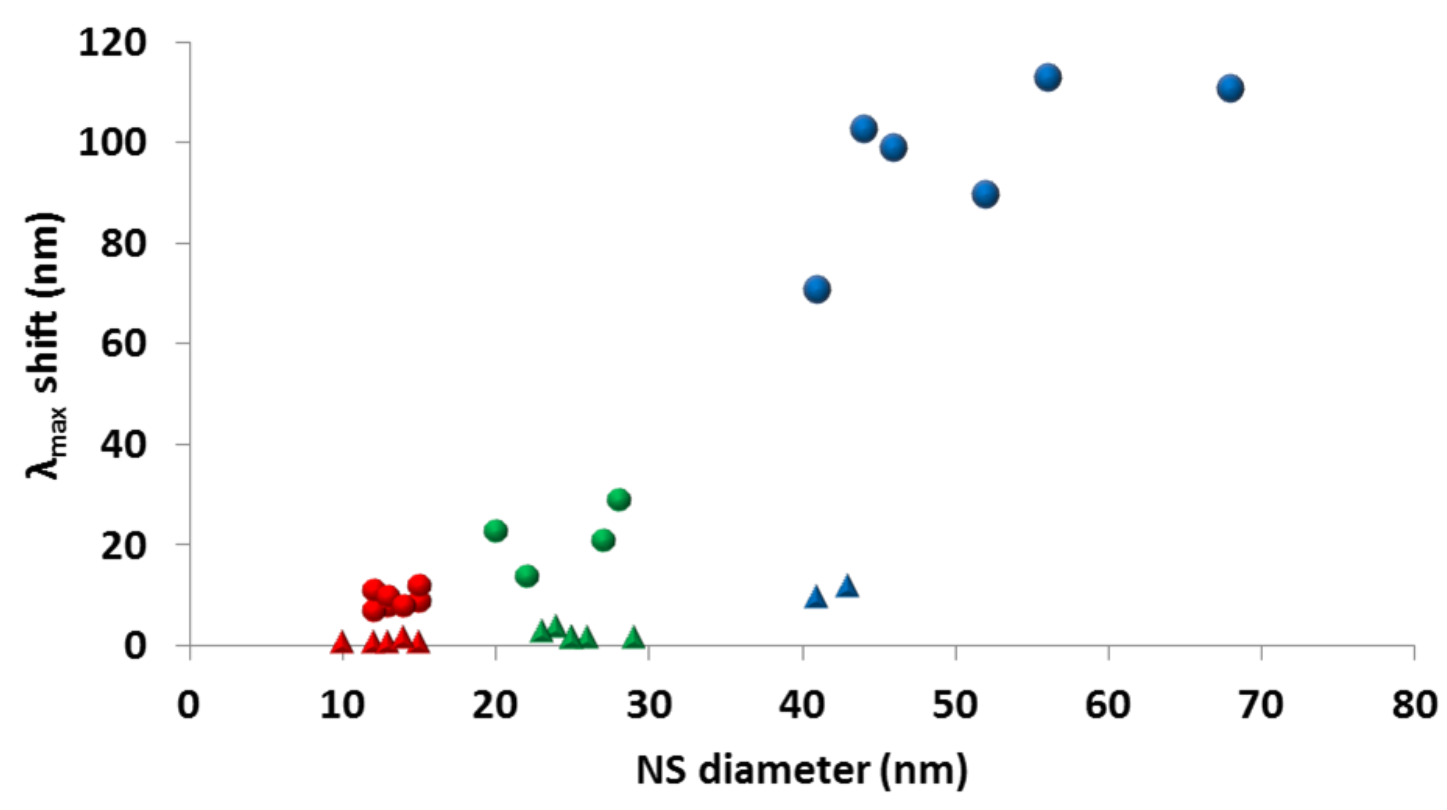

Figure 4.4. Dipolar plasmon shift as a function of NS size attached to an edge or terrace. NSs attached to the edges are represented by green circles $(13 \mathrm{~nm})$, blue circles $(24 \mathrm{~nm})$, and red circles $(51 \mathrm{~nm})$; NSs attached to the terrace are represented by green triangles $(13 \mathrm{~nm})$, blue triangles $(25 \mathrm{~nm})$, and red triangles $(42 \mathrm{~nm})$. 


\subsubsection{Multiple Big NSs Coupled to the Edge and Terraces of NPs}

Structures with multiple bigger sized NSs attached to a NP were also studied. Figure 4.5 A shows a triangular NP with an initial $\lambda_{\max }$ of $680 \mathrm{~nm}$ before NS attachment, which has three peaks after attachment of two Au NSs to the edge. The size of the two NSs is $36 \mathrm{~nm}$ and $38 \mathrm{~nm}$, respectively. The most red-shifted peak is at $800 \mathrm{~nm}$, which shifted positive $120 \mathrm{~nm}$ from $680 \mathrm{~nm}$. Another red-shifted peak is around $730 \mathrm{~nm}$, which red shifted $50 \mathrm{~nm}$ from its original positon. A blue-shifted shoulder peak is around 600 $\mathrm{nm}$. Similar to a single big NS attached, the LSPR band at $800 \mathrm{~nm}$ can be assigned to head-to-tail dipolar coupling of the NP and NSs parallel to the in-plane long-axis. We attribute the shoulder peak around $600 \mathrm{~nm}$ to $\sigma^{*}$ type dipolar coupling of the NP and NSs. The LSPR peak around $730 \mathrm{~nm}$ can be assigned to dipolar coupling of the NP and NS perpendicular to the long-axis, analogous to $\pi$ bond formation. In Figure 4.5 B, three aggregated NSs were attached to the terrace of a hexagonal NP with an initial $\lambda_{\max }$ of 692 nm. A red-shifted peak at $734 \mathrm{~nm}$ was observed after the attachment of NSs. Three NSs attached caused a red shift of $42 \mathrm{~nm}$ (21 nm per NS), which was much bigger than the shift caused by a single NS attached to the terrace of a NP (10-12 nm per NS). The

reason might be the synergistic effect observed in multiple smaller $(24 \mathrm{~nm}) \mathrm{NSs}$ or a simple aggregation effect of the NSs. 

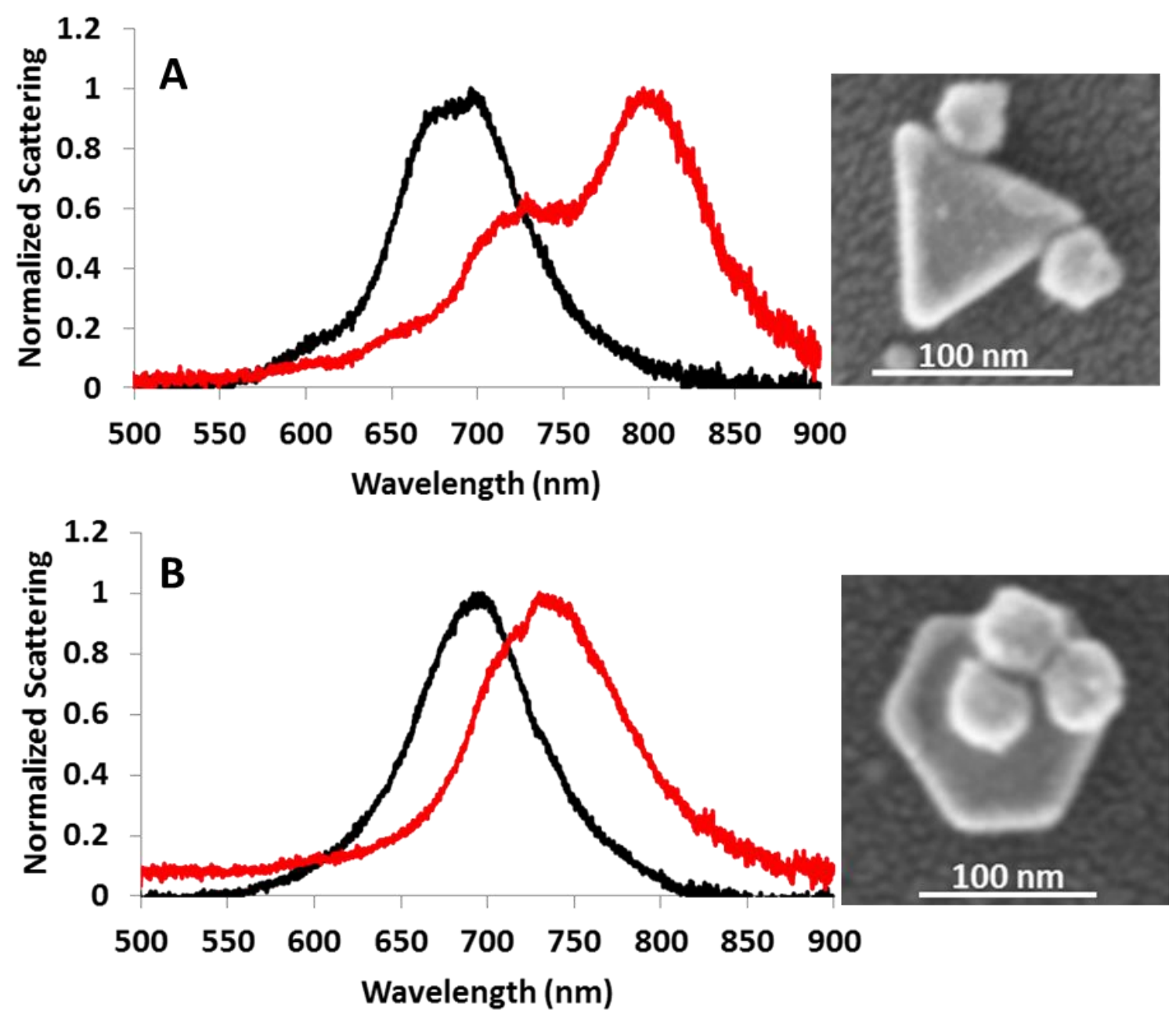

Figure 4.5. Dark-field scattering spectra of a single nanoplate before (black) and after (red) NS attachment on the edge (A) or terrace (B) and their SEM images to the right. Scale bars in SEM images are $100 \mathrm{~nm}$. 


\subsection{CONCLUSIONS}

Individual plasmonic heterodimers assembled by the ligand place-exchange method have been studied using resonant Rayleigh dark-field scattering spectroscopy. The coupling of shape mismatched NPs and NSs provides some unique optical properties highly dependent on the site of attachment and size of the NS. We find that the site of the NS attachment dictates the plasmonic response of the NP: when NSs attached on the edge, strong coupling with the NP results in a big red shift, whereas NSs attached on the terrace cause little perturbation of the NP plasmon resonance and results in small red shift. In the case of head-to-tail coupling of NP and NS, modifying the diameter of the NS changes not only the amount of the red shift but also the LSPR spectra of the assembly. First, the amount of red shift is not linear with the diameter of the NS. It increases nonlinearly with NS diameter at first and then reaches a saturation level with larger diameters, which fits well with a simple model describing the electric field strength of a NS as a function of the distance from the NS and the size. Second, the number of LSPR modes increases with increasing NS size. When light is polarized perpendicular to the long NP/NS axis, the NP and NS couple in a side-by-side configuration, which is much weaker relative to the coupling when light is polarized parallel to the long NP/NS axis. With large NSs, these two plasmon modes are easily distinguished from one another due to the large red shift for the parallel coupling. With the smaller NSs, these modes are not distinguishable and overlap into one broad peak. These unique optical properties related to shape asymmetric heterodimers can be exploited for 3D spatial sensing with nanometer resolution. 


\section{CHAPTER V}

\section{THE EFFECT OF LINKER ON THE PLASMONIC COUPLING BETWEEN GOLD NANOSPHERES AND GOLD NANOPLATES MEASURED AT THE SINGLE NANOSTRUCTURE LEVEL}

In this chapter, we describe the effect of the organic linker on plasmonic coupling of single or multiple $\mathrm{Au}$ nanospheres (NSs) onto a $\mathrm{Au}$ nanoplate (NP). The organic linker binds to both the NS(s) and the NP and brings them together (acting as a crosslinker), allowing for plasmonic coupling between the two nanostructures. In this work, we compared the plasmonic coupling of one or more Au NSs to a Au NP linked by cysteamine (Cys) molecules and those linked by 4-aminothiophenol (4-ATP). The red shift in the plasmon wavelength relative to the NP only was larger upon coupling with NSs and there were more plasmon modesobserved in the spectrum for NP/NS nanostructures coupled through Cys linkers as compared to 4-ATP. This is most likely due to the shorter length of the Cys linker. Because the linker length of Cys and 4-ATP is different, we were not able to distinguish between the length and chemical nature of the linker. 


\subsection{INTRODUCTION}

Bifunctional organic molecules such as 4-ATP and dithiols have been widely used in construction of well-defined 1D, 2D, or 3D assembled nanostructures. For example, Yoon and co-workers used ${ }^{147}$ dithiols of varied alkane chain length to couple small $\mathrm{Au}$ NS satellites to large Au NS cores. They found that that the near-field coupling, as determined by the LSPR band, had a strong dependence on the gap distance. As the length of the dithiol linker decreased from hexadecanedithiol (C16) to ethanedithiol (C2), the LSPR coupling band red-shifted, indicating the increase in their coupling strength. Novak et al. assembled $\mathrm{Ag}$ and $\mathrm{Au}$ nanoparticles with thioacetyl-teminated phenylacetylene linkers. In their study, thioacetyl-teminated phenylacetylene with varied aromatic ring numbers were used to bridge two Ag NSs. They observed a high-energy band appearing beside the dipole peak of a $\mathrm{Ag}$ dimer with decreasing length of the molecular bridge between the Ag dimer. ${ }^{148}$ Recently, more attention has been put on the nature of the organic linkers and their effect on plasmon coupling between nanoparticles. Yoon and coworkers investigated several different molecular functional groups on organic linkers and their effect on plasmon coupling. ${ }^{149}$ Organic linkers used in their research have a fixed - $\mathrm{SH}$ group on one terminal, an alkane chain in the middle, and a varied functional group $\left(-\mathrm{SH},-\mathrm{OH}, \mathrm{COOH}, \mathrm{CH}_{3}\right.$ etc.) on the other terminal. The different nature of the interactions between the functional group and Au nanoparticles caused subtle changes in the plasmon coupling band of the core-satellite nanoassemblies. Linkers with the SH group exhibited the biggest red-shift while the linker with the $\mathrm{CH}_{3}$ group showed the smallest shift. To our best knowledge, there is no study plasmon coupling of nanoparticles comparingan with linker comprised of an alkane chain in the middle as compared to an aromatic ring with the two terminals being the same. This 
could be interesting since there is a large difference between the electronic properties of an alkane chain and aromatic ring. Electrons in an aromatic ring are highly delocalized the linker is somewhat conductive while the electrons in an alkane chain are highly localized and the molecule is more insulating. Dithiols with aromatic rings such as 4,4'biphenyldithiol (BPD) are considered good candidates as conductors in molecular electronics applications. ${ }^{150}$ However, it is not well understood how the conductance of an organic linker affects the plasmon coupling of nanostructures linked by them. It is reasonable to believe that molecular conductance could affect the plasmon coupling considering that those plasmons involve the conducting electrons in the metal.

There are three possible ways that plasmonic coupling between two or more nanostructures can be altered through variation of the organic linker. The first is by a variation in the distance between the coupled structures. Since plasmonic coupling is exponentially dependent on nanostructure distance, this can be a strong effect. The shorter the linker, the stronger the coupling between the nanostructures. This is well known and has been studied previously and quantified in studies of molecular rulers with nanostructure dimers. ${ }^{147}$ The dielectric property of the organic linker can also have an effect on plasmonic coupling since the coupling is related to the interaction of the electric fields created by the dipoles forming at the metal nanostructure surface as the conducting electrons interact and become excited by electromagnetic radiation. In general, the higher the dielectric constant, the higher the plasmonic coupling observed. It is often difficult to distinguish between the effect of a changing dielectric property and the distance. A third possible effect on plasmonic coupling is when the molecule itself can be excited by light at a frequency similar to the plasmon frequency of the nanostructures. This can also lead to enhanced coupling. We chose to compare the Cys linker to 4-ATP 
because they both have a thiol group that binds to the Au NP and an amine group on the other end that binds to the NSs electrostatically as $\mathrm{NH}_{3}{ }^{+}$. Also, the Cys linker is a two carbon alkane chain in between the thiol and amine, while the 4-ATP contains a benzene ring between the thiol and amine with highly delocalized electrons. We believed that the delocalized electrons might improve plasmon coupling between the NP and NSs. Unfortunately the distance is not the same, making the analysis difficult.

In our work, we coupled one or more Au NSs to a Au NP through Cys and 4-ATP linkers and compared the near-field coupling of the nanostructures. Both molecules attach to the NP through a thiolate bond and to the NS electrostatically through the $\mathrm{NH}_{3}{ }^{+}$ group. The Cys has a two-carbon chain in the middle as compared to a benzene ring for the 4-ATP, which is clearly more conductive with delocalized electrons, which was thought to improve plasmonic coupling. The results actually showed greater plasmonic coupling for the Cys linker, which we attributed to the shorter length of the Cys linker, leading to the Au NS-NP distance being shorter. This dominated the plasmonic coupling in this case, making it impossible to study the effect of the alkane chain versus the benzene ring on plasmonic coupling. The experiment needs to be designed such that the linker distance is the same, but the group between is different to better understand the difference between an aromatic and alkane chain in plasmonic coupling. We can conclude that the benzene ring does not provide such enhanced coupling that it overcomes the effect of the distance, since the Cys linker shows stronger coupling. 


\subsection{EXPERIMENTAL}

The synthesis of the Au NPs and Au NSs, the preparation of the nanostructure assemblies via ligand place-exchange reactions and characterization by LSPR and SEM are described in Chapter II.

Ligand-exchange reactions were used to control the NS attachment location on the NP. In the previous chapters, the ligands involved in those experiments were 4-ATP and HT. In this chapter, Cys ligands were exchanged with HT to investigate the ligand effects on nanostructure coupling. The experimental conditions which work for 4-ATPHT exchange reactions did not work with Cys-HT exchange, when Cys was used to control the attachment location of NSs on a NP. A new set of experiments were designed and performed to explore the optimal conditions for Cys-HT exchange and controlled NS attachment.

We placed the glass/Au NPs sample in a $6 \mathrm{mM}$ ethanol solution of Cys overnight. We rinsed thoroughly with ethanol and dried under $\mathrm{N}_{2}$. The substrates were then immersed in a $10 \mathrm{~mL}$ solution of $\sim 25 \mathrm{~nm}$ diameter Au NSs for $30 \mathrm{~min}$, rinsed thoroughly with nanopure water, and dried under $\mathrm{N}_{2}$. The SEM image in Figure 5.1A shows that NSs aggregated on the NP surface. In order to avoid the aggregation of NSs, different soaking times were tested to reach an ideal coverage of NSs. It was found that a $5 \mathrm{~min}$ soaking time in Au NS solution provided a good coverage of NSs on a NP (Figure 5.1B). The above experiments allowed us to better understand the Cys linker compared to 4ATP. Even though they have the same terminal groups, Cys seems to have a stronger affinity to Au NSs than 4-ATP based on the results. The condition of the HT-Cys ligand place-exchange reaction was also explored to control the attachment location of NSs on the NP. 

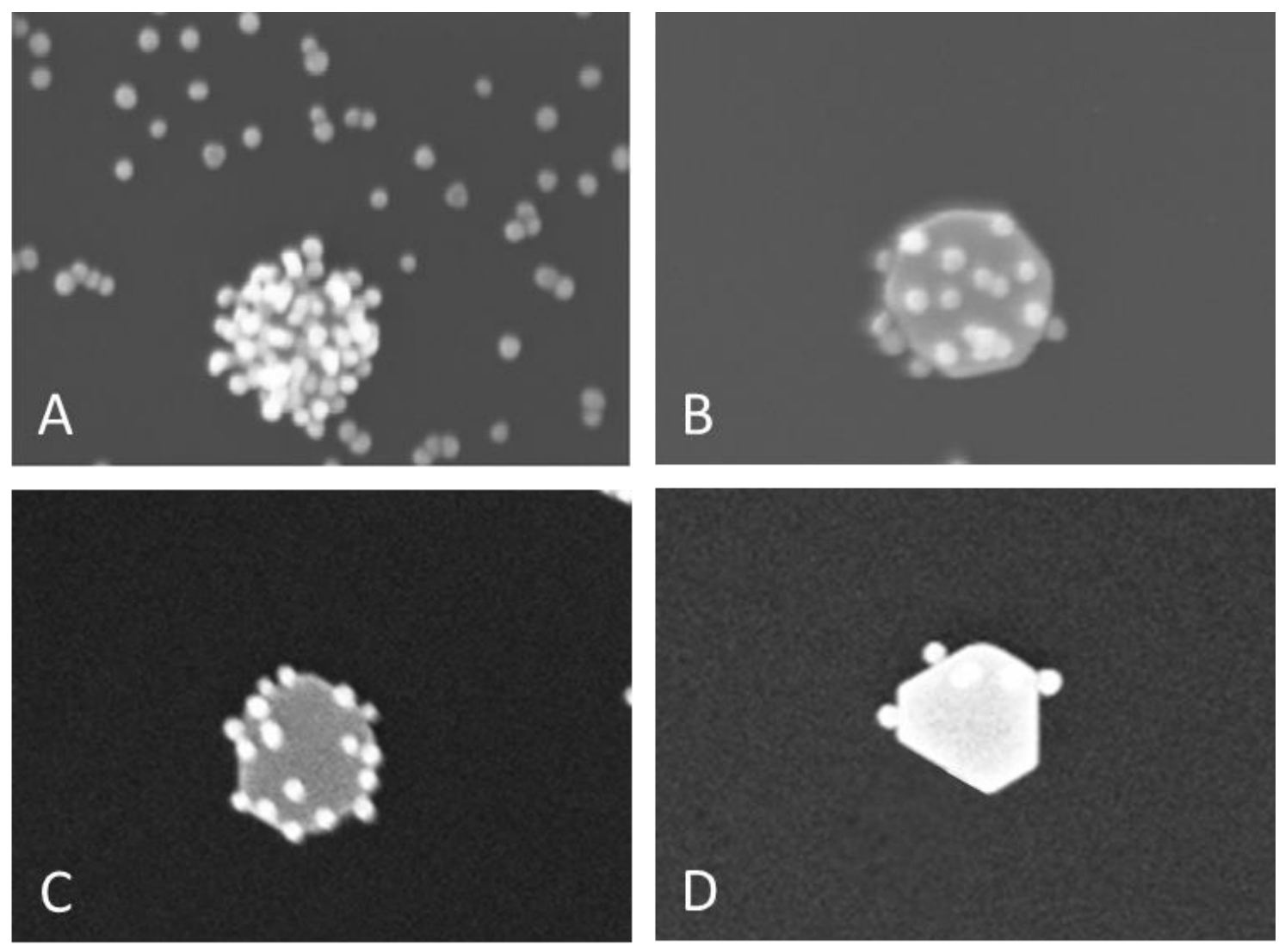

Figure 5.1. SEM images of $25 \mathrm{~nm}$ Au NSs attached onto a Au NP under different conditions. (A) $6 \mathrm{mM}$ Cys overnight and $30 \mathrm{~min}$ soaking in a Au NS solution. (B) $6 \mathrm{mM}$ Cys overnight and $5 \mathrm{~min}$ soaking in a Au NS solution. (C) HT-Cys ligand place-exchange for $1 \mathrm{~h}$ followed by $30 \mathrm{~min}$ of soaking in a Au NS solution. (D) HT-Cys ligand place-exchange for $1 \mathrm{~h}$ followed by $5 \mathrm{~min}$ of soaking in a Au NS solution. 
We placed the glass/Au NPs sample in a $1 \mathrm{mM}$ ethanol solution of HT overnight. We rinsed thoroughly with ethanol, dried under $\mathrm{N}_{2}$, and then exchanged the HT monolayer with Cys by placing the sample into a $6 \mathrm{mM}$ ethanol solution of Cys for 1 hour. We again rinsed thoroughly with ethanol and dried under $\mathrm{N}_{2}$. The substrates were then immersed in a $10 \mathrm{~mL}$ solution of $\sim 25 \mathrm{~nm}$ diameter Au NSs for $30 \mathrm{~min}$, rinsed thoroughly with nanopure water, and dried under $\mathrm{N}_{2}$. Figure $5.1 \mathrm{C}$ and $\mathrm{D}$ shows that $\mathrm{NSs}$ selectively attached to NP edge sites after a 5 min soaking time following a $1 \mathrm{~h} \mathrm{HT}$-Cys ligand place-exchange.

\subsection{RESULTS AND DISCUSSION}

\subsubsection{The Coupling of Au NSs to Hexagonal Au NPs through Cys and 4-ATP}

\section{Linkers}

Figure 5.2 shows two hexagonal Au NPs with one $24 \mathrm{~nm}$ diameter $\mathrm{Au}$ NS attached to a vertex site via coupling through a Cys linker (Frame A) and a 4-ATP linker (Frame B). The scattering spectra before NS attachment displayed one main peak due to the dipolar plasmon mode of the hexagonal NP. The $\lambda_{\max }$ was $788 \mathrm{~nm}$ and $678 \mathrm{~nm}$ in

Frames A and B, respectively, indicating that the hexagon in Frame A had a higher aspect ratio (AR). After coupling of a Au NS to the Au NP via a Cys linker, two peaks appear in the scattering spectrum. The most red-shifted peak appeared at $824 \mathrm{~nm}$ (peak 1) and another less red-shifted peak appeared at $800 \mathrm{~nm}$ (peak 2). In addition there was a blueshifted shoulder peak around $600 \mathrm{~nm}$ (peak 3). Peak 1 red-shifted $36 \mathrm{~nm}$ relative to the original NP while peak 2 red-shifted $12 \mathrm{~nm}$. The largest shift of peak 1 is due to dipolar $\sigma$-type coupling of the NS and NP with light polarized along the long axis of the coupled 

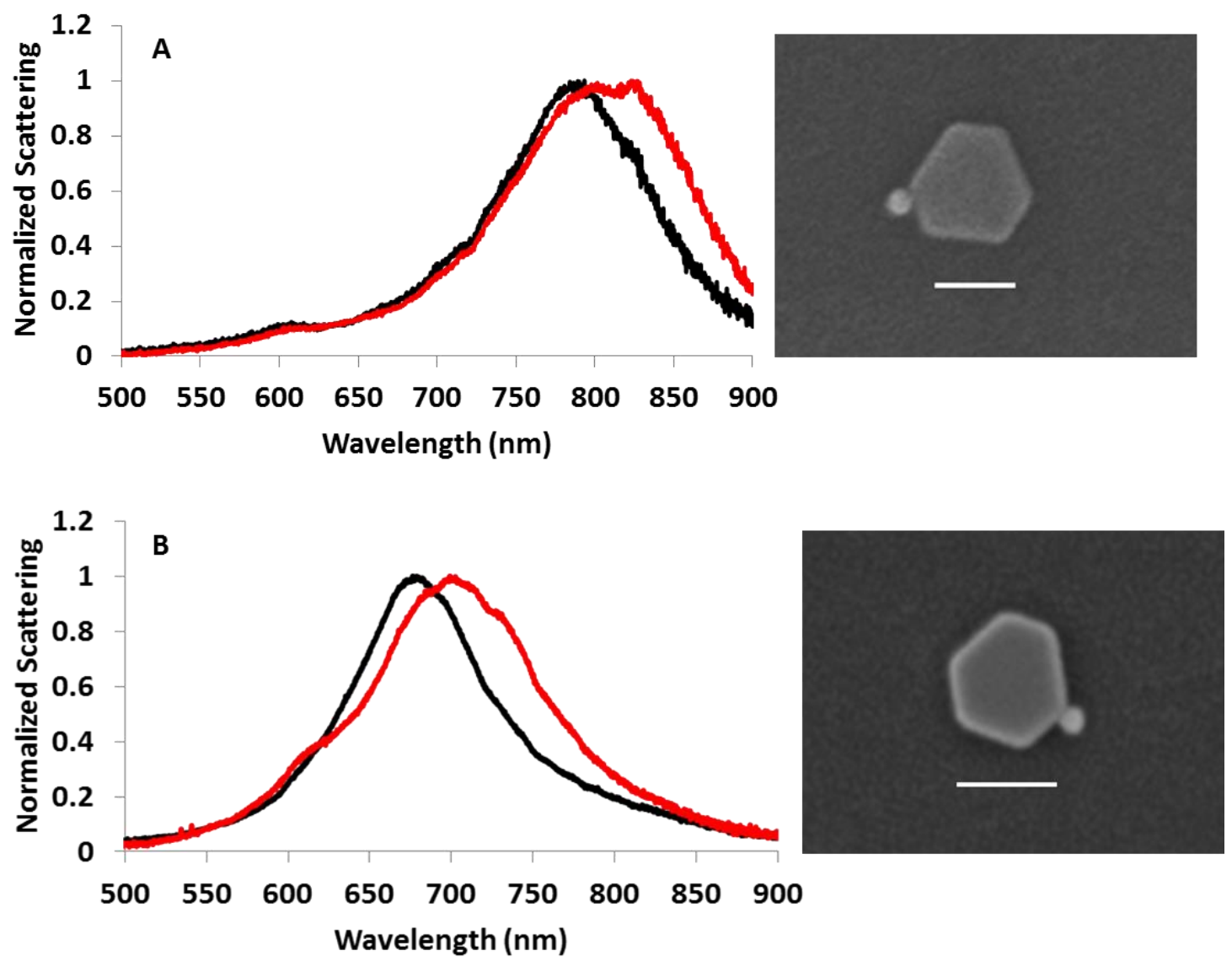

Figure 5.2. Scattering spectrum of a hexagonal Au NP before (black line) and after (red line) attachment of a Au NS on an vertex site of the NP vis a (A) Cys linker and (B) 4-ATP linker. The SEM image of the exact structure is shown to the right of the spectra. Scale bars in SEM images are $100 \mathrm{~nm}$. 
structure. The less red-shifted peak 2 is due to interaction with light polarized in the direction of the short axis, or perpendicular to the NP/NS axis. The shift is smaller due to weaker coupling with the NS along this direction. When 4-ATP molecules were used to selectively attach a $27 \mathrm{~nm}$ Au NS to the vertex site of the hexagonal NP there was one peak red-shifted peak to $699 \mathrm{~nm}$ (21 nm shifts) and one blue-shifted shoulder peak near $600 \mathrm{~nm}$. The blue shifted peak is the same as that for the NP/NS structure linked with Cys. The main red-shifted peak is due to dipolar coupling along the lone NP/NS axis. Since the shift is only $21 \mathrm{~nm}$, we do not observe the third peak due to light polarized along the NP short axis. This peak overlaps with the $21 \mathrm{~nm}$ shifted dipolar peak, leading to one red-shifted broadened peak after NS attachment. We attribute the larger red-shift with the Cys linker to the smaller size and subsequent shorter distance between the NP and NS. This larger red shift allowed the observation of two plasmon modes corresponding to light polarized along the long axis of the NP/NS structure and light polarized along the short axis of the NP/NS structure. When the shift is smaller in the case of 4-ATP, we observe one broad peak instead.

Figure 5.3 shows a schematic representation of the two different linker molecules used in this study. The distance from the $\mathrm{N}$ to the $\mathrm{S}$ in the molecule is about $0.5 \mathrm{~nm}$ for the Cys linker and $0.7 \mathrm{~nm}$ for 4-ATP. This molecular length should correlate with the NS-NP distance and hence the coupling strength. We believe this is the reason for the larger dipolar red shift due to head-to-tail coupling along the long axis, which is shifted large enough to also observe the peak due to polarization along the short axis of the coupled structure with the Cys linker. A comparison between our data and simulations performed by the Schatz group show good agreement, where the LSPR band red shifted and a high-energy shoulder peak grew in with a decrease in the interparticle distance 

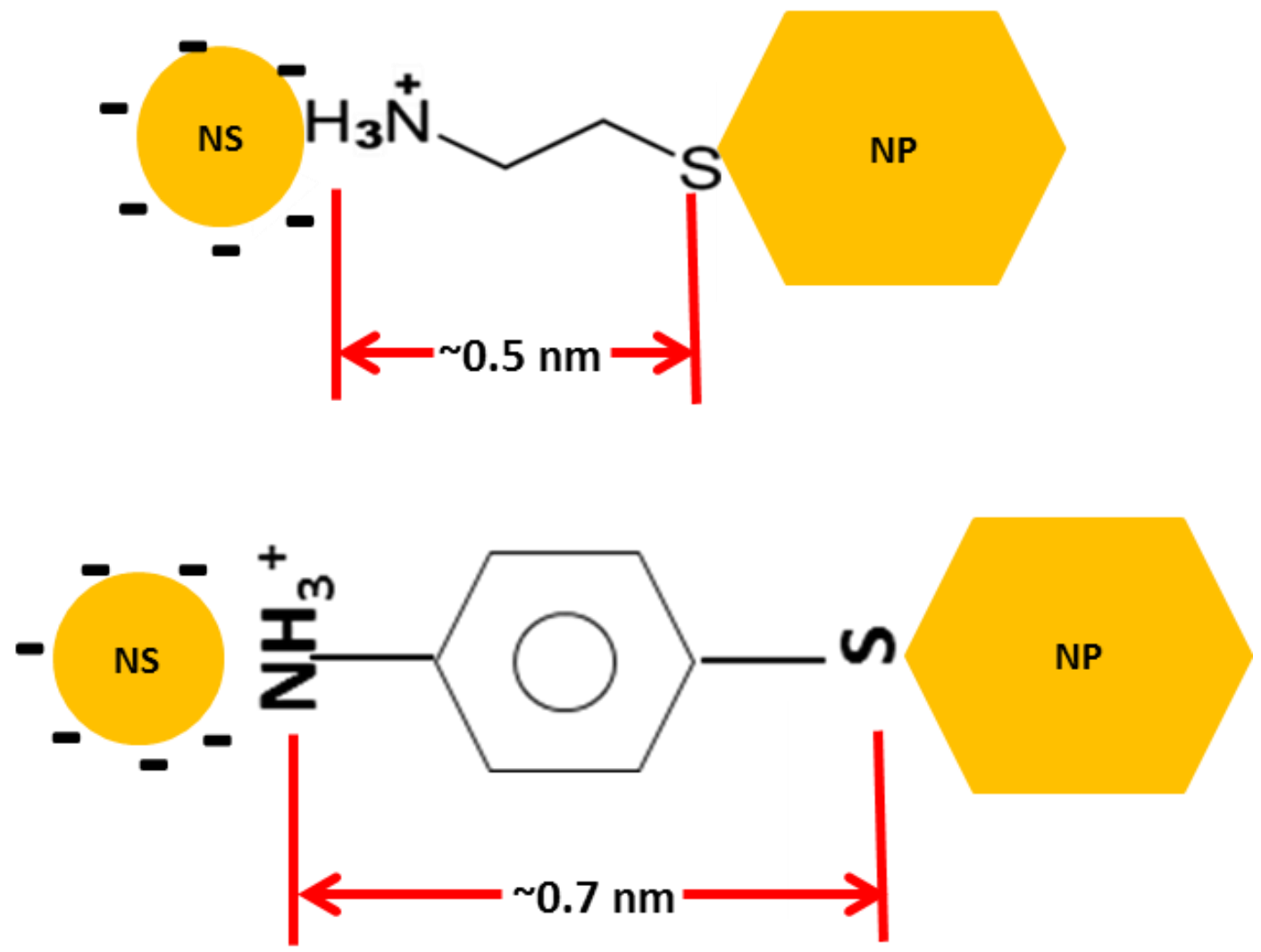

Figure 5.3. Illustration of the interparticle distance between a NS and NP linked by (A) Cys molecule and (B) 4-ATP molecule. 
between Ag NS dimers. ${ }^{151}$ However, there is still uncertainty in the exact distance between the Au NS and NP using these two different linker molecules. The molecules could be extended perpendicular from the surface at a maximum length or have different degrees of tilting relative to the surface normal, which could drastically affect the NS-NP distance. The interparticle distance with a Cys molecule standing up straight on a $\mathrm{Au}$ surface is 0.8 angstrom longer than the molecule tilting $60^{\circ}$ relative to the Au surface, for example. $^{152}$

Figure 5.4 shows the resulting scattering spectrum following attachment of two and three NSs to a hexagonal NP using the Cys and 4-ATP linker, respectively. Prior to attachment through Cys, the spectrum shows one main symmetric peak with a $\lambda_{\max }$ of 668 nm (Frame A). Following the attachment of the two NSs through Cys, the spectrum of the coupled nanostructure consists of three peaks, including a red-shifted peak at $750 \mathrm{~nm}$ (peak 1), a second red-shifted peak at $702 \mathrm{~nm}$ (peak 2), and a blue-shifted shoulder peak around $600 \mathrm{~nm}$ (peak 3). The presence of three peaks is similar to the coupling of one NS in Figure 5.2, except that the two red-shifted peaks are shifted more in the Cys case ( $82 \mathrm{~nm}$ as compared to $36 \mathrm{~nm}$ for peak 1 and $34 \mathrm{~nm}$ compared to $12 \mathrm{~nm}$ for peak 2). The shift for two coupled NSs is larger than twice the shift for one NS. The average red shift caused by each NS when two were attached was $41 \mathrm{~nm}$ for peak 1 and $17 \mathrm{~nm}$ for peak 2 , slightly larger than the shift of $36 \mathrm{~nm}$ and $12 \mathrm{~nm}$ for one NS attached. In the case of 4ATP as the linker for three attached NS, the NP prior to coupling displayed a $\lambda_{\max }$ of 656 nm. After attachment of three NSs, there were only two peaks in the resulted LSPR spectrum, including one red-shifted peak at $725 \mathrm{~nm}$ and one blue-shifted shoulder peak around $600 \mathrm{~nm}$. The total shift was $69 \mathrm{~nm}$, or an average of $23 \mathrm{~nm}$ per NS, which is significantly smaller than the shift per particle with the Cys linker. We attribute the 

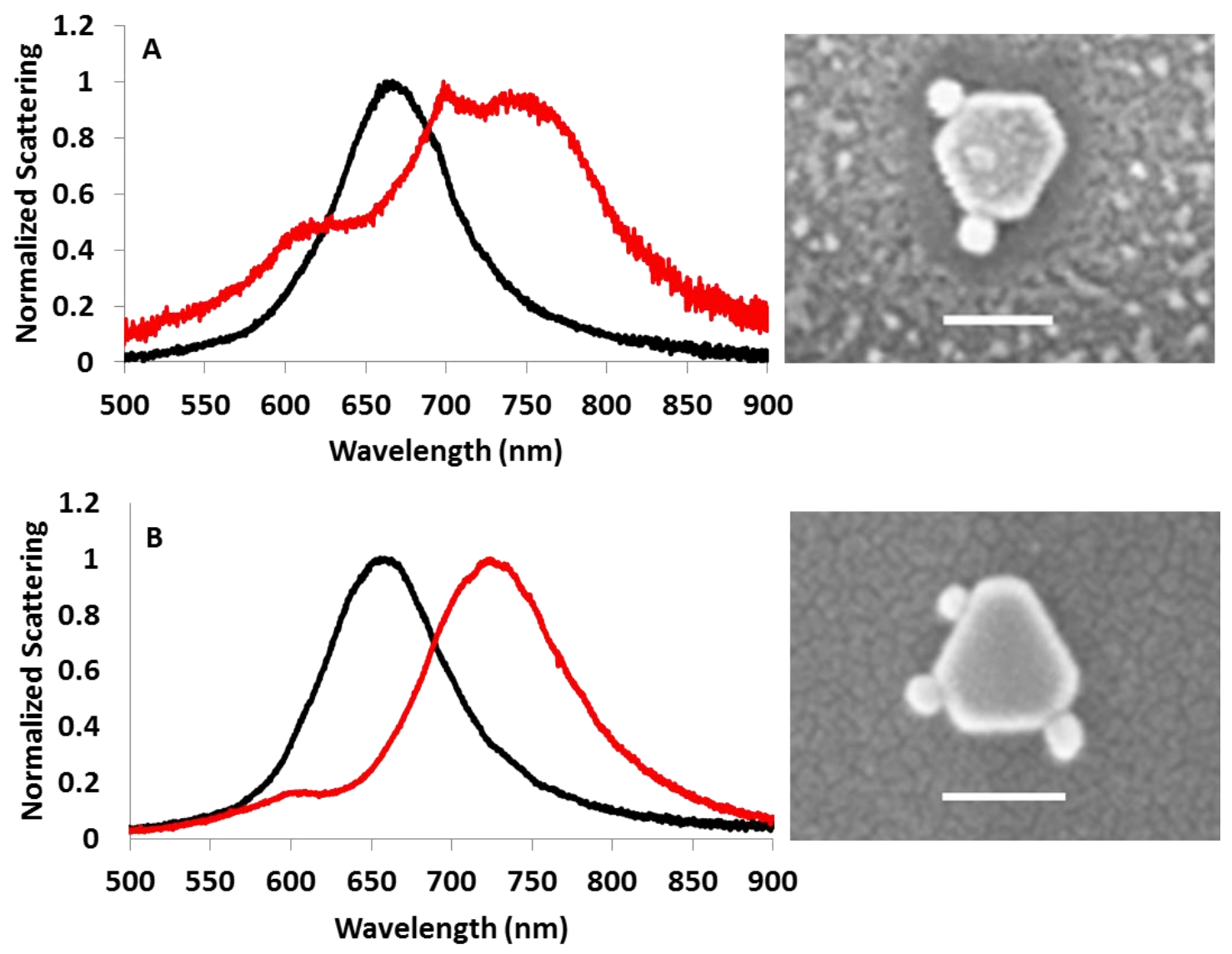

Figure 5.4. Dark-field light scattering spectrum of an approximately hexagonal Au NP before (black line) and after (red line) attachment of (A) two Au NSs to the edge site via Cys linkers and (B) three Au NSs on the edge site via 4-ATP linkers. The SEM images show the exact structures used to obtain the data. Scale bars in SEM images are $100 \mathrm{~nm}$. 
bigger shift for Cys to the closer Au NP/NS distance as already discussed. Two redshifted peaks are not visible with the 4-ATP linker because of the fairly symmetric distribution of NSs around the NP. This leads to a similar red-shift for all light polarization directions, leading to one broad peak instead of multiple peaks. The unique synergistic effect in the shift in $\lambda_{\max }$ observed in the 4-ATP experiments described in Chapter 3 was also observed with Cys linkers. ${ }^{146}$ For example, the attachment of a single NS to a NP resulted in a $36 \mathrm{~nm}$ red-shift while the attachment of two NSs to the edge site of a Au NP resulted in a $82 \mathrm{~nm}$ red-shift, amounting to a shift of $41 \mathrm{~nm}$ per attached NS. Both linkers showed a blue-shifted peak at $\sim 600 \mathrm{~nm}$, but the band was more intense as the number of NSs increased and for the Cys linker, consistent with the shorter linker distance.

\subsubsection{The Coupling of Au NSs to Circular Au NPs through Cys and 4-ATP Linkers}

Figure 5.5 shows the dark-field light scattering spectra of two circular Au NPs before and after the attachment of one 20-30 nm diameter Au NS through a Cys and 4ATP linker in Frames A and B, respectively. The circular Au NP before NS attachment via Cys linker had one main peak with a $\lambda_{\max }$ of $650 \mathrm{~nm}$ and another slightly visible shoulder peak near $690 \mathrm{~nm}$. There are likely two peaks in the before spectrum because the circular disk is not completely symmetric. It is elongated in one direction, giving a more red-shifted peak for interactions with light polarized along that direction. There appears to be four highly overlapping LSPR peaks after NS attachment, including a redshifted peak at $735 \mathrm{~nm}$ (peak 1), a second red-shifted peak at $688 \mathrm{~nm}$ (peak 2), a third red-shifted peak at $664 \mathrm{~nm}$ (peak 3), and a blue-shifted shoulder peak around $600 \mathrm{~nm}$ 
(peak 4). The peak at $735 \mathrm{~nm}$ (peak 1) seems to have red-shifted from the peak at 690 $\mathrm{nm}$ in the original spectrum (total shift of $45 \mathrm{~nm}$ ). It is likely due to the NS coupling along the elongated axis of the circular NP. The other two red-shifted peaks (peaks 2 and 3) came from the original broad peak at $650 \mathrm{~nm}$, but are now more distinct because they shifted by different amounts. Those two peaks shifted by $38 \mathrm{~nm}$ and $14 \mathrm{~nm}$ and correspond to different amounts of coupling along different NP axes. The peak at 600 $\mathrm{nm}$ is blue-shifted by $50 \mathrm{~nm}$ and assigned as described previously for the other shaped NPs. The presence of the multiple peaks and intense band at $600 \mathrm{~nm}$ is again due to the close spacing between the NS and NP with the Cys linker. In the case of NS coupling to the edge of the circular NP in Frame B through the 4-ATP linker, the NP before coupling exhibited a $\lambda_{\max }$ of $653 \mathrm{~nm}$. After NS attachment, there was really only one main peak in the dark-field scattering spectrum, a red-shifted peak at $667 \mathrm{~nm}$ (14 nm shifts). The blueshifted shoulder peak at $600 \mathrm{~nm}$ observed for many of the other structures is barely noticeable, if at all. As shown for hexagonal NPs, the $600 \mathrm{~nm}$ peak was not nearly as intense or distinguishable with the 4-ATP linker compared to the Cys linker. Also, there is only one broad red-shifted peak after NS coupling, which actually consists of different red-shifted peaks underneath it due to different polarization directions (since we use unpolarized light) relative to the long NS-NP axis, but are not different enough to be distinguishable because of the longer 4-ATP linker and less overall coupling strength compared to Cys. Another interesting observation is that the main dipolar plasmon mode shifted by $14 \mathrm{~nm}$ for one NS coupled to a circular NP, whereas it shifted by $>20 \mathrm{~nm}$ for one NS coupled to a hexagonal NP. This is due to the stronger electric field strength surrounding the edges and corners of the hexagonal shaped NP. 

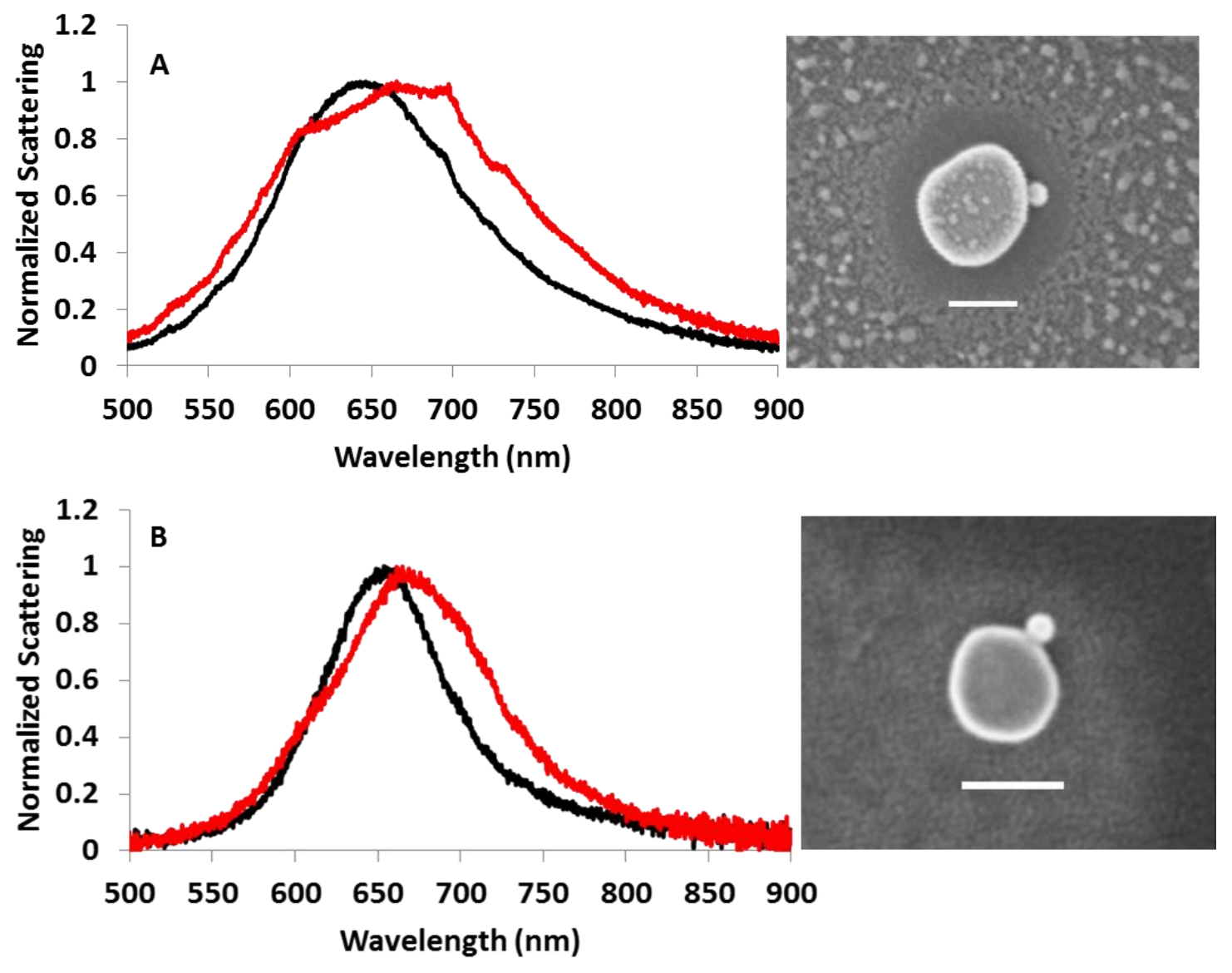

Figure 5.5. Dark-field light scattering spectrum of a circular Au NP before (black line) and after (red line) attachment of a Au NS on the edge site of the NP vis (A) Cys linkers and (B) 4-ATP linkers. The SEM images of the exact structure studied are shown to the right of the spectra. Scale bars in SEM images are $100 \mathrm{~nm}$. 


\subsubsection{The Effect of Coupling Location on the Plasmonic Properties of NSs Attached to Triangular NPs}

In Chapter III, we discussed the effect of coupling a Au NS to the vertex/edge site of a hexagonal NP as compared to coupling to the terrace site, showing a dramatic difference in coupling strength with unpolarized light. In that study, we used 4-ATP linkers and could not distinguish between NS coupling to a vertex site versus an edge site. Figure 5.6 shows the dark-field scattering spectra of two triangular NPs before and after attachment of a 20-30 nm Au NS to the edge and vertex site through a Cys linker, where significant difference in coupling occurred between these two locations. Specifically, Figure 5.6 A shows a triangular NP with an initial $\lambda_{\max }$ of $731 \mathrm{~nm}$ before $\mathrm{Au}$ NS attachment. After attachment of a $29 \mathrm{~nm} \mathrm{Au} \mathrm{NS} \mathrm{through} \mathrm{Cys,} \mathrm{the} \mathrm{spectrum} \mathrm{contains}$ three LSPR peaks, including a red-shifted peak at $806 \mathrm{~nm}$ (peak 1), another red-shifted peak at $734 \mathrm{~nm}$ (peak 2), and a blue-shifted shoulder peak around $600 \mathrm{~nm}$ (peak 3). Peak 1 red-shifted $75 \mathrm{~nm}$ relative to the original triangular NP LSPR peak while peak 2 redshifted $3 \mathrm{~nm}$. Figure 5.6 B shows the spectrum of a triangular Au NP before and after attachment of an $18 \mathrm{~nm}$ Au NS to the edge site of a triangular NP through Cys linkers. The NP had one original $\lambda_{\max }$ at $718 \mathrm{~nm}$ and also three peaks after NS edge attachment. It consisted of one red-shifted peak at $732 \mathrm{~nm}$ (peak 1), another red-shifted peak at 719 $\mathrm{nm}$ (peak 2), and one blue-shifted shoulder peak round $600 \mathrm{~nm}$ (peak 3). Peak 1 redshifted by $14 \mathrm{~nm}$ and peak 2 red-shifted by $1 \mathrm{~nm}$ compared to the original LSPR peak of the NP. The red shift of peak 2 in both vertex and edge attached samples was similar (3 $\mathrm{nm}$ vs $1 \mathrm{~nm}$ ), representing the interaction of light polarized perpendicular to the NP/NS long axis. However, the magnitude of the red shift for peak 1 (along the NP/NS long axis) was 5 times larger in the case of vertex attachment compared to edge attachment 

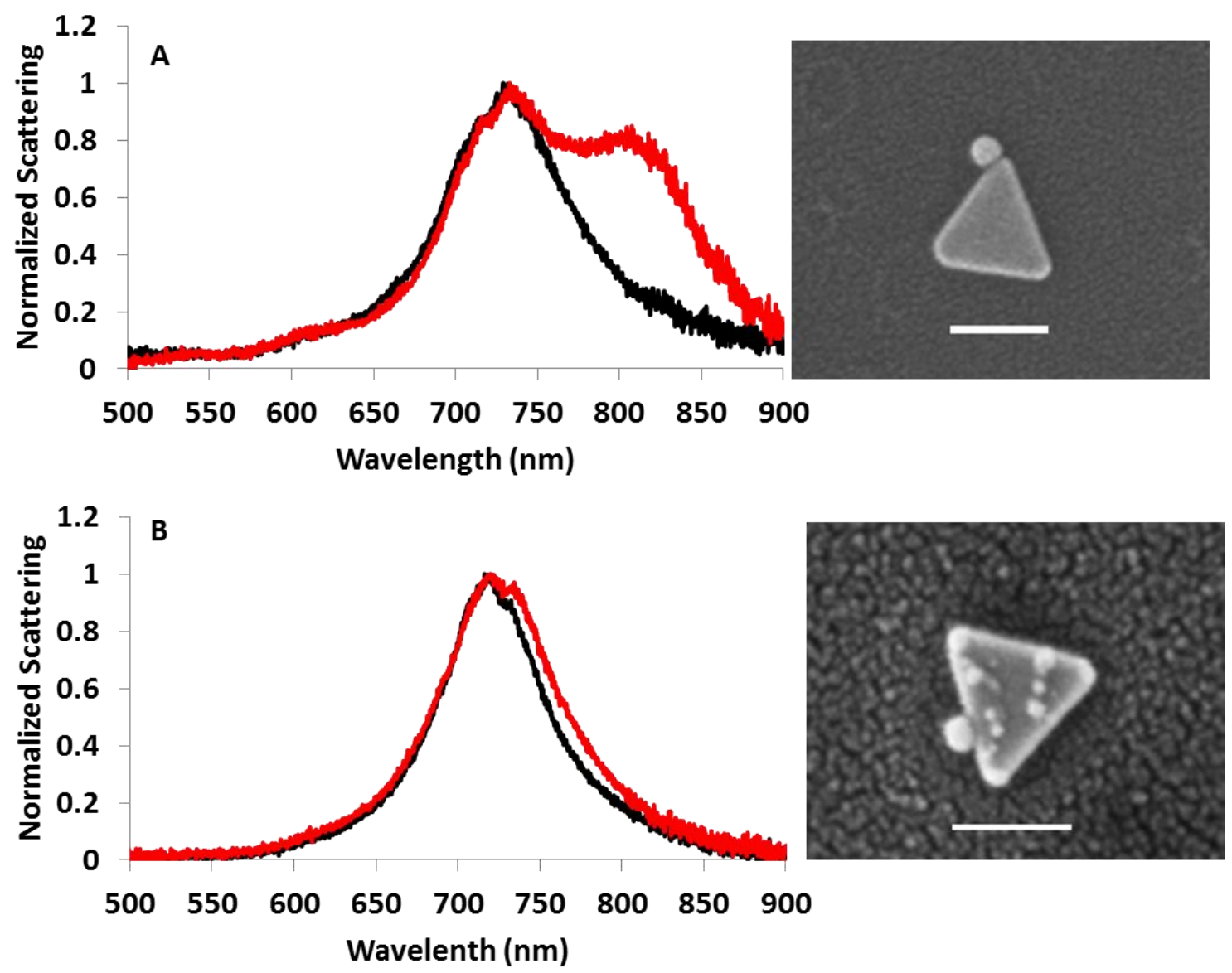

Figure 5.6. Dark-field light scattering spectrum of a triangular Au NP before (black line) and after (red line) attachment of a Au NS on a (A) vertex site and (B) the edge site of the NP via Cys linkers. The SEM images to the right show the actual coupled nanostructures. Scale bars in SEM images are $100 \mathrm{~nm}$. 
(75 nm vs $14 \mathrm{~nm}$ ). In our previous studies, ${ }^{146}$ simulation of the electric field around the different regions of a hexagonal NP showed site-dependent electric field strengths in the order of the vertex $>$ edge $>$ terrace, which explains why the NS attached on the vertex site of a NP resulted in a bigger shift compared to attachment on the edge site for the triangular NP, which would show a similar electric field site dependence. Our results here agree well with the simulation of the polarization difference between the vertex and edge sites of a triangular NP. The surprising result is that we did not observe this difference with the 4-ATP linker on hexagonal or triangular NPs. This suggests that a shorter linker may be better at distinguishing optically between the edge and vertex sites of a well-defined shaped nanostructure, such as triangular NPs. It is important to note that the NS was larger on the vertex site $(29 \mathrm{~nm})$ compared to the NS on the edge site (18 $\mathrm{nm})$, but this should not account for the larger difference in the shift (75 nm vs $14 \mathrm{~nm})$.

\subsection{CONCLUSIONS}

In this chapter, we described the optical light scattering properties of different $\mathrm{Au}$ NP/NS coupled structures linked together by two different organic linkers (Cys and 4ATP). The results show a large difference in the spectra of coupled NP/NS structures with the different linkers. In the spectrum of Cys-linked NP/NS dimer, there are three LSPR peaks, including a distinct higher-energy plasmon mode in addition to different red-shifted dipolar coupling modes. The 4-ATP linked structure does not have an as distinguishable blue-shifted peak or a second red-shifted peak. The appearance of these modes with the Cys linker is attributed to the shorter interparticle distance with Cys compared to 4-ATP, although we cannot rule out possible differences in the dielectric 
properties or electronic properties of the linkers. A synergistic effect observed previously with 4-ATP linkers and multiple binding NSs was also observed here with Cys linkers, but to a greater extent. In addition, with the Cys linker we were able to distinguish between the coupling of a NS onto the edge and vertex site of a triangular NP that was not observed with the 4-ATP linker. This research provides new and important information in the plasmonic coupling of Au NSs to Au NPs via different organic linkers. The information learned here could be utilized to better control the optical properties of coupled nanostructures and develop a strategy for optical sensing with 3D spatial resolution. Although we were not able to directly compare the effect of a aromatic group and an alkyl group placed between the two coupled nanostructures, due to the difference in spacing, it is clear that the aromatic group did not cause significantly enhanced coupling since the Cys linker showed stronger coupling. 
CHAPTER VI

\section{EFFECT OF NANOPLATE SHAPE ON LIGHT SCATTERING PROPERTIES AND SENSITIVTY TO CHANGES IN THE REFRACTIVE INDEX OF THE ENVIRONMENT}

The aspect ratio (AR) of individual triangular, hexagonal and circular $\mathrm{Au}$ nanoplates (NP) was determined based on the height and maximum width as measured by Atomic Force Microscopy (AFM) and Scanning Electron Microscopy (SEM), respectively, and correlated to the wavelength of maximum scattering $\left(\lambda_{\max }\right)$ as measured by dark-field scattering spectroscopy/microscopy. For all shapes, the $\lambda_{\max }$ increases linearly with increasing AR. The $\lambda_{\max }$ is more red-shifted and more sensitive to AR in the order of triangles $>$ hexagons $>$ cirlces. The sensitivity of the $\lambda_{\max }$ to the refractive index (RI) of the global environment, known as Refractive Index (RI) sensitivity $\left(\mathrm{S}_{0}\right)$, followed the order of triangles $(\sim 250 \mathrm{~nm} / \mathrm{RIU})>$ hexagons $(\sim 220 \mathrm{~nm} / \mathrm{RIU})>$ circles ( $\sim 150 \mathrm{~nm} / \mathrm{RIU})$, based on the $\lambda_{\max }$ values in air and 2-propanol. Dark-field microscopy images showed a clear change in color of the NPs in the two different RI media. 


\subsection{INTRODUCTION}

The optical properties and RI sensitivity of nanoparticles depends on a number of factors such as the size, shape, composition, ${ }^{106,108,113}$ and nanoparticle-nanoparticle interactions (coupling or aggregation). ${ }^{153,154}$ A number of studies have been conducted to characterize the RI sensitivity of different shaped nanoparticles including nanospheres (NSs), NRs, cubes, plates, bipyramids, and nanostars (or nanobranches). ${ }^{106,113,155}$ In general, nanostructures with very sharp points, such as nanostars and nanobranches, have shown the largest RI sensitivities, due to the large electromagnetic field enhancement at these sharp regions. Au NPs are desirable platforms for understanding the fundamentals of LSPR sensing because have different regions with very different plasmon properties, including sharp vertex and edge sites with larger electromagnetic field enhancement compared to the smooth terraces. The smooth morphology of the terrace makes it relatively easy to image large molecules, such as proteins, and other nanostructures attached to these different regions. For applications, the different regions could be exploited to study molecular dynamics and to develop 3D spatial nanosensors.

LSPR sensing of individual nanostructures has several benefits over measurements from an ensemble of nanostructures, including narrower LSPR bands, higher sensitivity, and the ability to observe structures with unique behavior. The first RI sensitivity study of a triangular NP was reported by the Van Duyne group. ${ }^{104}$ They reported that the dipole plasmon resonance peak of a Ag NP had a RI sensitivity of 205 $\mathrm{nm} / \mathrm{RIU}$. There are only a few studies comparing the RI sensitivity of NPs with other shapes such as rods and spheres. For example, the Van Duyne group reported that the RI

sensitivity of a single Ag nanoparticle follow the order of rod $>$ prism $>$ sphere. ${ }^{112}$ 
However, only the shape of the triangular NP, fabricated by nanosphere lithography (NSL), was confirmed by microscopy. The geometry of the NR and NS was based on the LSPR maximum wavelength, line shape, and polarization dependence of the scattering spectra instead of microscopy. Another study showed that a single Au NR has a higher RI sensitivity than a circular NP. ${ }^{113}$ In their study, both the shape of NRs and nanodiscs (or circular NP) was confirmed by high resolution SEM images. While some studies of NPs exist on RI sensitivity at the single nanoparticle level, there has been no report systemic study on the RI sensitivity of NPs with different geometric shapes.

In this chapter, we compare the RI sensitivity of triangular, hexagonal, and circular Au NPs. In our NP synthesis, we naturally have a distribution of triangular, hexagonal, and circular NPs on the surface. Since we are studying the nanostructures at the single particle level, we are able to study all three shapes from the same sample. This alleviates the need to controllably synthesize one particular shape with high yield. In our synthesis, hexagons make up about $90 \%$, triangles $10 \%$, and circles $<1 \%$. We synthesized the NPs on glass slides containing numbered markers as described earlier, in order to correlate the dark-field microscopy images and LSPR scattering spectra with the AFM and SEM images. The combination of height and width information of single NPs allowed us to correlate the AR with the $\lambda_{\max }$ for the three-different shaped NPs. We found that the $\lambda_{\max }$ increased with increasing AR for all NPs which agrees with other studies. ${ }^{64}$ However, the absolute $\lambda_{\max }$ value and sensitivity of $\lambda_{\max }$ on the AR varied for the different shapes. In addition, we measured and compared the RI sensitivity of each shape at the single particle level. While there have been comparisons in the RI sensitivity for various shaped $\mathrm{Au}$ nanostructures reported in the literature previously, there are no studies comparing different shaped NPs at the single particle level. Our results show that 
triangular NPs have a higher RI sensitivity, followed by hexagonal NPs, and then circular NPs. This study will provide useful information to build sensitive detection strategies based on LSPR properties.

\subsection{EXPERIMENTAL}

The synthesis and purification of the Au NPs and characterization by SEM, AFM, and Dark-field Microscopy/Spectroscopy are described in Chapter II.

\subsection{RESULTS AND DISCUSSION}

\subsubsection{LSPR Correlated to Aspect Ratio of Individual Au NPs}

The optical properties of nanoparticles have a strong dependence on their dimensions, especially with regard to the ratio of their height to width, or aspect ratio (AR) The AR of a NS is 1 and other anisotropic shaped nanoparticles are larger than 1 . The relationship between the AR of NPs and their maximum scattering wavelength $\left(\lambda_{\max }\right)$ has been studied for three different shaped NPs, including triangle, hexagon, and circle.

Figure 6.1 shows the dark-field scattering spectrum, SEM image and crosssectional AFM image for each NP. For example, the edge length was $125 \mathrm{~nm}$ and the corresponding height was $38 \mathrm{~nm}$ for a triangular NP (Figure 6.1A). The AR of this triangular NP was 3.3 and the $\lambda_{\max }$ was $665 \mathrm{~nm}$. The maximum width and height of seven individual triangular NPs were obtained along with the dark-field scattering spectrum. The $\lambda_{\max }$, corresponding to the in-plane dipole peak of the NP, was plotted as a function of AR as shown in Figure 6.2. The well-defined trends in the peak positions just noted are 


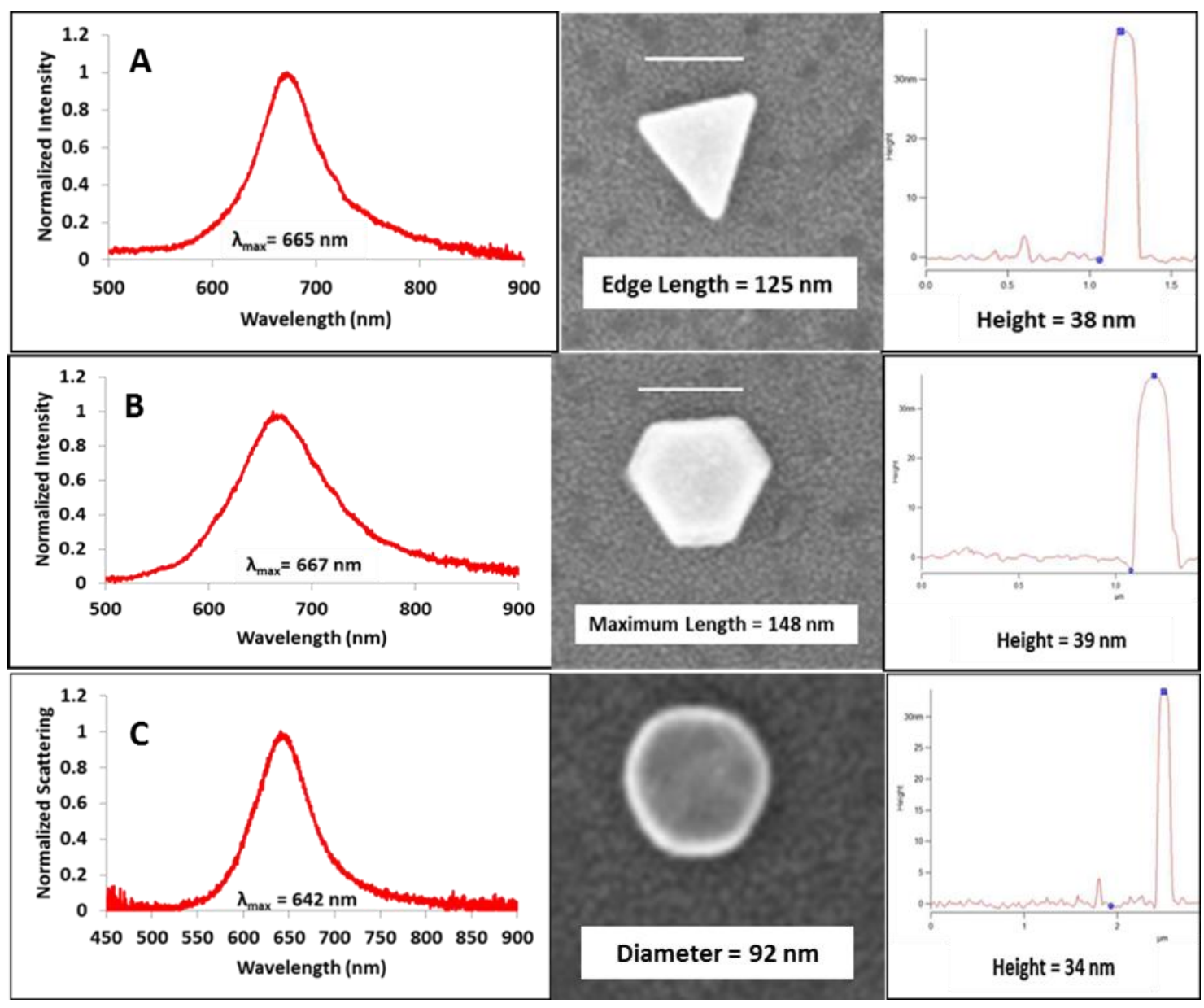

Figure 6.1. Dark-field light scattering spectrum (left panel), SEM image (middle panel), and cross-section AFM image (right panel) of an individual triangular Au NP (A), hexagonal NP (B), and circular NP (C). The scale bar in the SEM image is $100 \mathrm{~nm}$. 


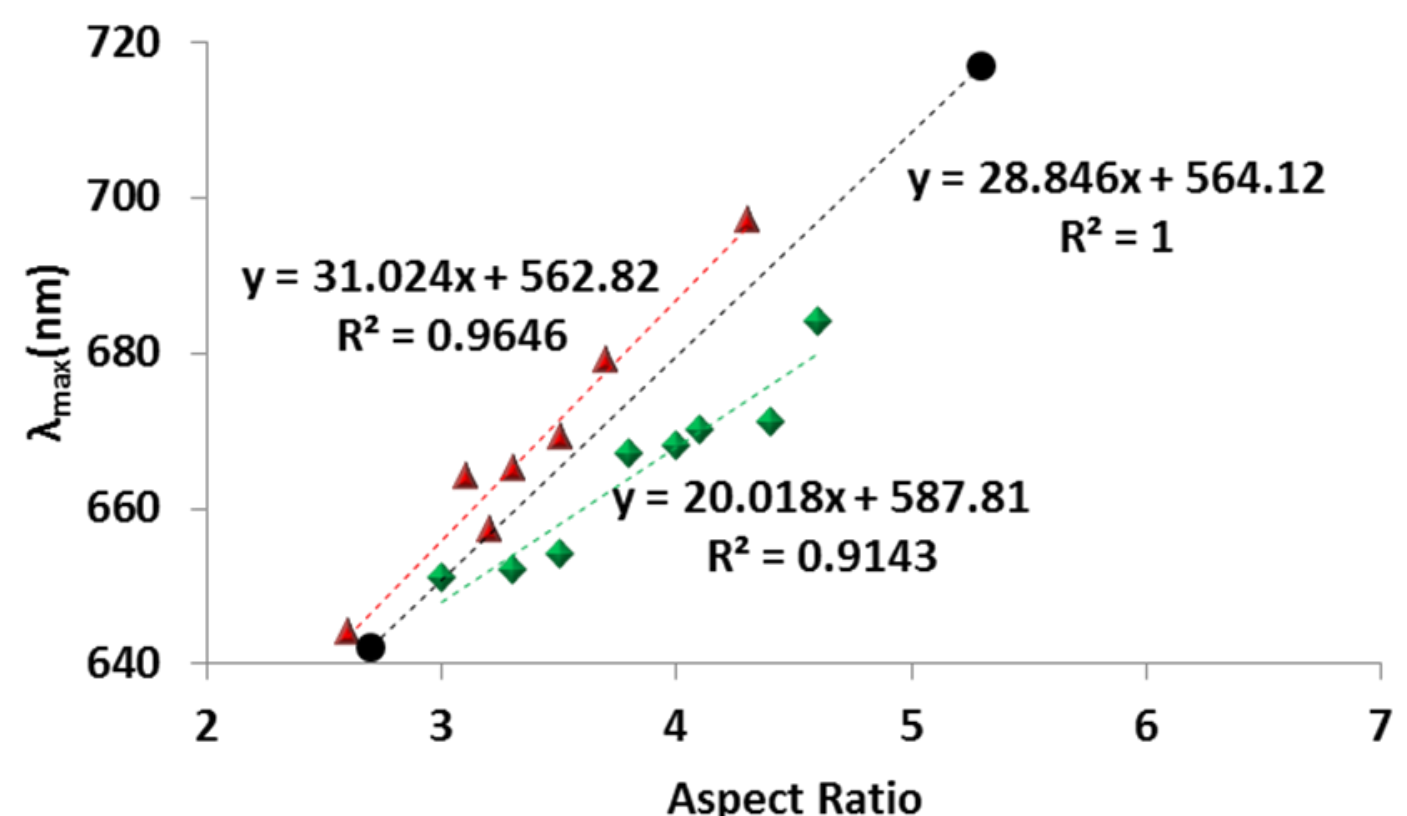

Figure 6.2. Relationship between the LSPR dipole peak position $\left(\lambda_{\max }\right)$ and aspect ratio of triangular (red triangles), hexagonal (green squares), and circular (black circles) NPs. 
strongly correlated to the nanoparticle dimensions. The LSPR dipole peak increased linearly with AR with a slope of $31 \mathrm{~nm} / \mathrm{AR}$ unit. The trace in the figure has a correlation coefficient greater than 0.96 indicating good fits. The in-plane modes dominate the extinction because of the bigger edge length $(>100 \mathrm{~nm})$ and smaller aspect ratio $(<7)$. Our experiment results agree closely with Schatz theoretical calculation. ${ }^{156}$ Schatz and coworkers theoretically calculated the scattering spectrum of individual $\mathrm{Au}$ prisms in aqueous solution with different edge length and thickness. They found a general increase in the wavelength of $\lambda_{\max }$ as the AR of the prism increased. However, no experimental data was provided for verification. According to their calculation, the smallest prism in water solution with an $\mathrm{AR}=2.5$ has a dipole peak at 673 . If $\mathrm{AR}=2.5$ replaces the $\mathrm{X}$ in the equation of Figure 5.4, the $\lambda_{\max }$ will be at 643 . The $30 \mathrm{~nm}$ differences maybe cause by the difference dielectric environment. The bottom of our NPs was attached to glass substrates and their surface was exposed in air. In the theoretical calculation, all their NPs were considered to be immersed in water.

The maximum width of a hexagonal NP was $148 \mathrm{~nm}$ and the corresponding height was $39 \mathrm{~nm}$ (Figure 6.1B). The resulting aspect ratio was 3.8 with a $\lambda_{\max }$ of 667 nm. Totally, edge length and height and corresponding dark-field scattering spectra of eight individual hexagonal NPs were obtained. A similar trend in the peak and nanoparticle dimensions was observed. The wavelength corresponding to the in-plane dipole peak increased linearly with aspect ratio (20 nm/AR unit), as can be seen in Figure 6.2. To our best knowledge, there has been no study on the relationship of $\lambda_{\max }$ and AR for hexagonal NPs. 
Circular NPs or nanodiscs have been wideley synthesized and used for fundemental studies of nanoparticle optical properties as well as sensing applications. Compared to triangular and hexagonal NPs, circular NPs have no sharp edges. Figure 6.1C shows the dark-field scattering spectrum of a circular Au NP along with the corresponding SEM and AFM cross-sectional image. The diameter of the circle was 92 $\mathrm{nm}$ and the corresponding height was $34 \mathrm{~nm}$. The resulting AR was 2.7 with a $\lambda_{\max }$ value of $642 \mathrm{~nm}$. There was only two data points for circular NPs. The limited number of circular NPs came from our modified seed-mediated growth method for NP synthesis. A statistical calculation showed that among 32 NPs, there were only 2 circular NPs. Unfortunately, there were only two good circular NPs among the collected NPs. Even with two data points we still can see the trend that $\lambda_{\max }$ increases with increasing $A R$ of circular NPs (Figure 6.2). The slope determined by these two data points was $28 \mathrm{~nm} / \mathrm{AR}$ unit. However, this value was not expected due to the limited number of data points. The expected order of linear regression slope should be triangle $>$ hexagon $>$ circle.

\subsubsection{DDA Simulation}

The discrete dipole approximation (DDA) method was used for simulation of the LSPR scattering spectra of Au NPs. A triangular NP which has an edge length of $110 \mathrm{~nm}$ and a thickness of $35 \mathrm{~nm}$ was simulated with a $111 \mathrm{~nm}$ long x $35 \mathrm{~nm}$ thick NP (Figure 6.3). The simulation employed unpolarized light for excitation to match the experimental conditions. The DDA simulated scattering spectrum with Lorentzian fit shows close agreement with the experiment. The simulated value of $657 \mathrm{~nm}$ is slightly blue shifted by 
$6 \mathrm{~nm}$ compared to the experimental value with a similar maximum length $(110 \mathrm{~nm})$ in experimental.

Figure 6.4 compares the experimental spectrum to a simulated spectrum of a hexagonal NP of similar dimensions. The experimental NP had a long-axis length of 136 $\mathrm{nm}$ and a thickness of $33 \mathrm{~nm}$. Simulations were performed for a hexagonal NP with a long-axis length of $137 \mathrm{~nm}$ and a thickness of $31 \mathrm{~nm}$. We obtained the $\lambda_{\max }$ value of 642 $\mathrm{nm}$ from simulation data. The difference between the simulated $\lambda_{\max }$ value and experimental value may come from the different curvature of the hexagonal NP used in the simulation and compared to the one in the experiment. As we can see from the SEM image, the tips of chemical synthesized NP are not as perfectly sharp as those in the simulation.

\subsubsection{Bulk Refractive Index Sensing of Different Shaped NPs}

Using the darkfield microscope system and strategy we have developed to study individual NPs, ${ }^{146}$ we first collected LSPR spectra of several individual NPs in air $(\mathrm{RI}=1.00)$ (Figure 6.5A), and then covered those NPs with IPA (RI=1.37) and collected their spectra again. After application of the IPA we observed a significant color change in each of the NPs (Figure 6.5B). The thickness of the IPA layer is much greater than the dimensions of any of the NPs and can be considered to form a new bulk dielectric environment. Note that the NPs initially red in color shifted to gray while paricles intially green in color shifted to red when comparing Figure 6.5A and Figure 6.5B. A graph showing the initial spectrum (black line) and the red-shifted spectrum (red line) of a typical red NP, pointed out by the white arrow in Figure $6.5 \mathrm{~A}$ and $6.5 \mathrm{~B}$, is displayed in 

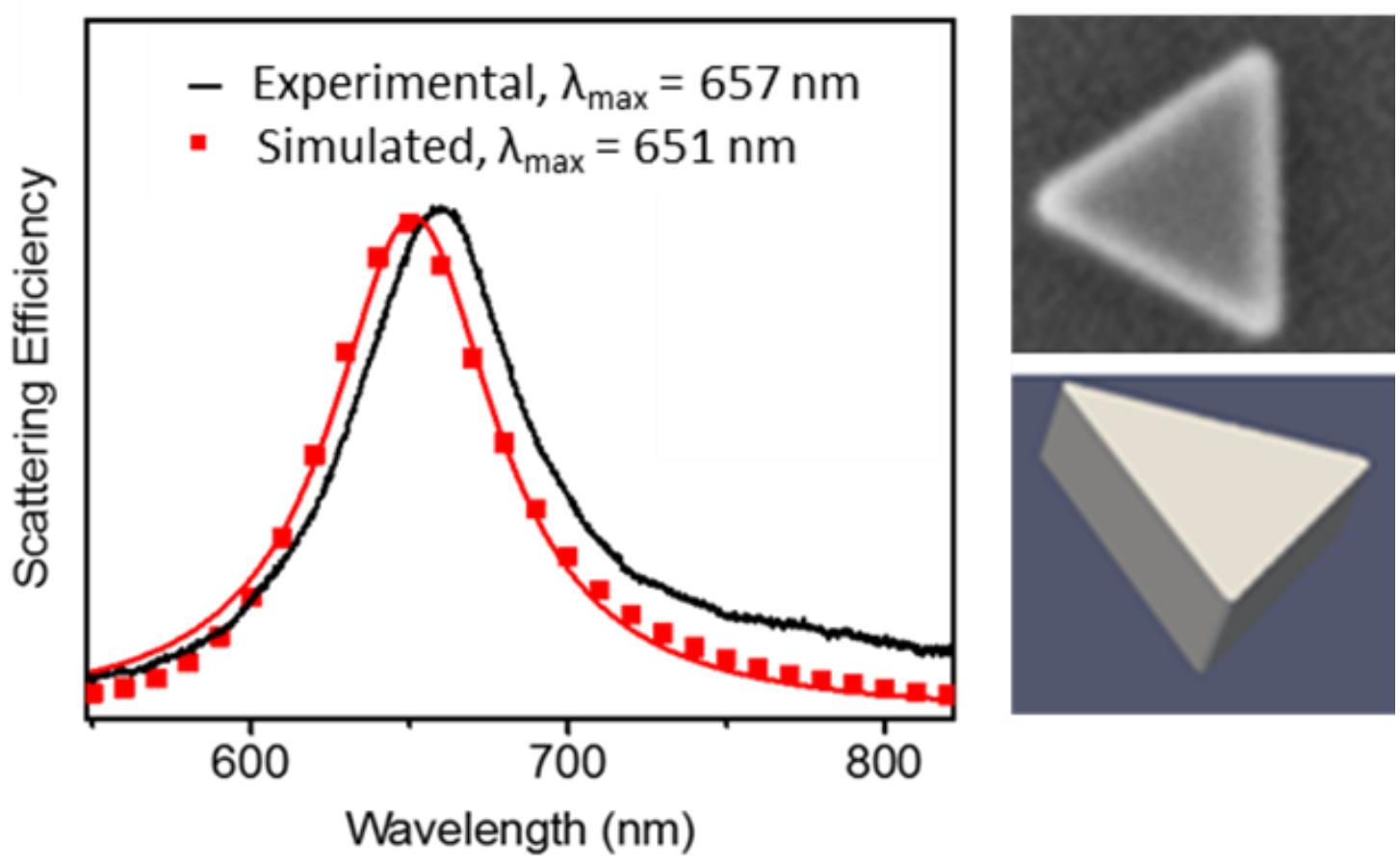

Figure 6.3. LSPR scattering spectrum of a single triangular Au nanoplate obtained via dark-field spectroscopy (black curve) and its DDA simulated scattering spectrum (red data points). SEM image (upper right) and simulated structure (bottom right) are also provided. 

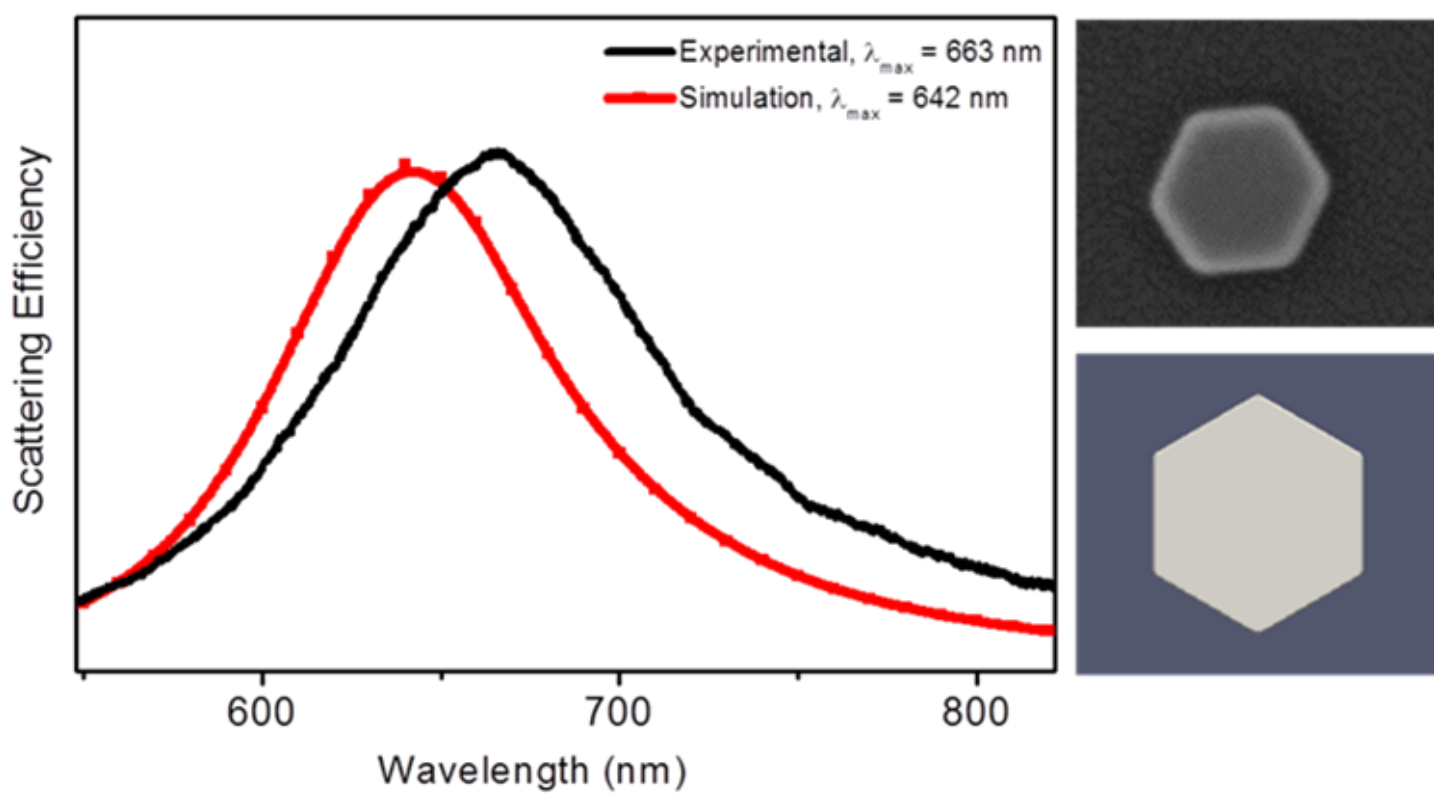

Figure 6.4. LSPR scattering spectrum of a single hexagonal Au nanoplate obtained via dark-field spectroscopy (black curve) and its DDA simulated scattering spectrum (red data points). The SEM image (upper right) and simulated structure (bottom right) are also provided to the right. 
Figure 6.5C. The $\lambda_{\max }$ of this red NP shifted $94 \mathrm{~nm}$ in the presence of bulk IPA. After removal of the IPA layer and drying, the LSPR scattering spectrum of the NPs shifted back to the original position. The nanostructure shown by a white arrow in Figure $6.5 \mathrm{~A}$ and $6.5 \mathrm{~B}$ is a triangualr NP which has a RI sensitivity of $247 \mathrm{~nm} / \mathrm{RIU}$.

Of the 32 NPs studied, hexagonal NPs accounted for 69\%, triangular NPs accounted for $25 \%$, and circular NPs $6 \%$. However, only a very small portion of each NP has a perfect shape. For example, some triangular NPs have rounded or truncated tips. All of those factors limited the number of useful data points. After careful selection, three triangular NPs, four hexagonal, and two circular NPs were chosen to study the RI sensitivity. Table 6.1, Table 6.2 and Table 6.3 lists the $\lambda_{\max }$ before and after covering with IPA, the $\lambda_{\max }$ shifts, RI sensitivity, and figure of merit (FOM) for each NP. The FOM is defined as the relative shift of the LSPR divided by the full width at halfmaximum (fwhm) of the LSPR peak as described in Chapter 1. The FOM serves as the standard for assessing a nanoparticle's sensing potential because FOM takes the spectral line width into consideration. Some nanoparticles exhibiting very large LSPR shifts have very broad LSPR peaks. A narrow spectral line width is desirable since a smaller change in signal is easier to detect as the spectral width decreases. For example, a triangular NP P3 in Table 6.1 has a bigger red shift of $97 \mathrm{~nm}$ compared to a $94 \mathrm{~nm}$ shift in P1. However, when taking the peak line width into consideration, P3 only has a FOM value of 2.76 which is much smaller than 3.51 of P1 since the LSPR band of P1 is much narrower. 

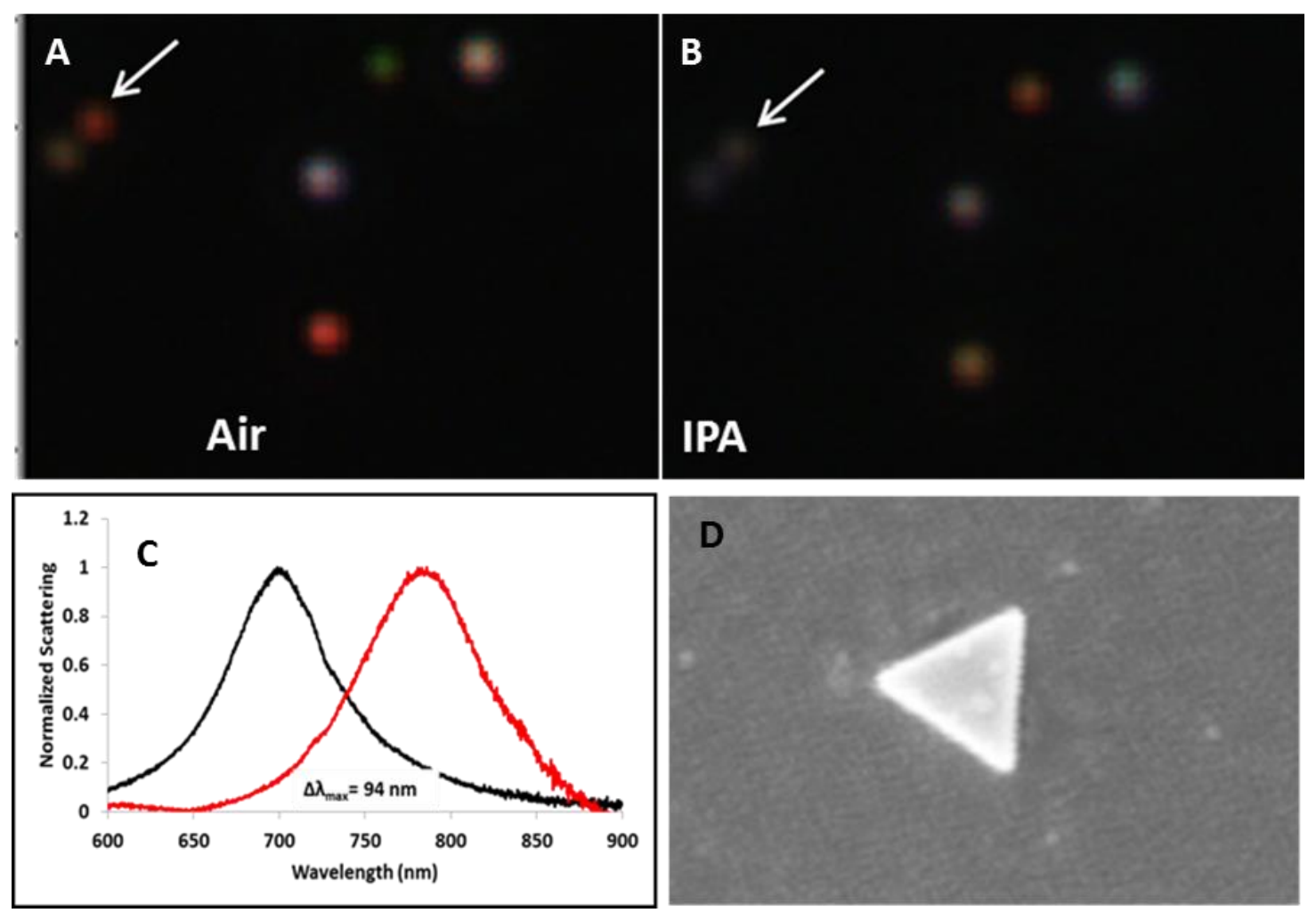

Figure 6.5. Correlated single-NP dark-field spectroscopy/electron microscopy. (A) Dark-field microscopy image of 6 individual Au nanostructures on a glass slide in air. (B) Dark-field microscopy image of the same 6 individual Au nanostructures on the same glass slide in IPA. (C) The light scattering spectrum of the Au NP pointed out by the white arrows in (A) and (B). (D) An SEM image of the NP pointed by the white arrows in (A) and (B). 
The results in Tables 6.1, 6.2, and 6.3 provide an important opportunity to study the influence of NP shape on RI sensitivity. For instance, the average FOM value from each triangular, hexagonal, and circular NP is $3.18 \pm 0.41,2.48 \pm 0.08$, and $1.83 \pm 0.32$, respectively. Based only on FOM, it seems that the RI sensitivity follows the order of triangular NPs $>$ hexagonal NPs $>$ circular NPs. When comparing individual NPs, we can draw the same conclusion. For two NPs labeled P2 in both Table 6.1 and 6.2, one triangular NP P2 has a $\lambda_{\max }$ value of $660 \mathrm{~nm}$ which is close to the $\lambda_{\max }$ of the hexagonal NP P2 at $662 \mathrm{~nm}$. However, the FOM of the triangular NP is 3.22 and that of the hexagonal NP is 2.52 . We conclude that the triangular shaped NPs are more sensitive to the refractive index changes compared to hexagonal or circular shapes. When comparing a hexagonal NP P1 in Table 6.2 with a $\lambda_{\max }$ of $679 \mathrm{~nm}$ to a circular NP P2 in Table 6.3 with a $\lambda_{\max }$ of $678 \mathrm{~nm}$, the hexagonal NP has a bigger FOM of 2.51 compared to 2.06 of the circular NP. The sensitivity of the NPs follows the order of triangles $>$ hexagons $>$ circle.

The sensitivity difference between these three types of NPs might come from the sharpness degree of their tips. To be more specific, the curvature of triangle tips are $60^{\circ}$ while the curvature of hexagon tips are doubled $120^{\circ}$ which means the tips of a triangle are sharper than the tips of a hexagon. For less sharp circular NPs, its curvature is $360^{\circ}$, which triples the degree of the hexagonal NP. Many theoretical and experimental studies have shown that the electromagnetic field is much more intense in sharp regions of the nanostructures. 


\begin{tabular}{|l|l|l|l|l|l|l|}
\hline $\begin{array}{l}\text { Triangle } \\
\text { Nanoplate }\end{array}$ & $\begin{array}{l}\lambda \max \text { in } \\
\text { Air }(\mathrm{nm})\end{array}$ & $\begin{array}{l}\lambda \max \text { in } \\
\text { IPA }(\mathrm{nm})\end{array}$ & $\begin{array}{l}\text { Shift } \\
(\mathrm{nm})\end{array}$ & $\begin{array}{l}\text { Sensitivity } \\
(\mathrm{nm} / \mathrm{RIU})\end{array}$ & FOM & $\begin{array}{l}\text { SEM } \\
\text { Images }\end{array}$ \\
\hline P1 & 690 & 784 & 94 & 247 & 3.57 & \\
\hline P2 & 660 & 753 & 93 & 245 & 3.22 & \\
\hline P3 & 656 & 753 & 97 & 255 & 2.76 & \\
\hline
\end{tabular}

Table 6.1. LSPR $\lambda_{\max }$ shift data and pertinent experimental environmental sensitivity parameters for dipole resonance of three different triangular NPs.

\begin{tabular}{|l|l|l|l|l|l|l|}
\hline $\begin{array}{l}\text { Hexagon } \\
\text { Nanoplate }\end{array}$ & $\begin{array}{l}\lambda \text { max in } \\
\text { Air }(\mathrm{nm})\end{array}$ & $\begin{array}{l}\lambda \text { max in } \\
\text { IPA }(\mathrm{nm})\end{array}$ & $\begin{array}{l}\text { Shift } \\
(\mathrm{nm})\end{array}$ & $\begin{array}{l}\text { Sensitivity } \\
(\mathrm{nm} / \mathrm{RIU})\end{array}$ & FOM & $\begin{array}{l}\text { SEM } \\
\text { Images }\end{array}$ \\
\hline P1 & 679 & 760 & 81 & 213 & 2.51 & \\
\hline P2 & 662 & 751 & 89 & 234 & 2.56 & \\
\hline P3 & 653 & 745 & 92 & 242 & 2.52 & \\
\hline P4 & 667 & 745 & 78 & 205 & 2.35 & \\
\hline
\end{tabular}

Table 6.2. LSPR $\lambda_{\max }$ shift data and pertinent experimental environmental sensitivity parameters for dipole resonance of four different hexagonal NPs.

\begin{tabular}{|l|l|l|l|l|l|l|}
\hline $\begin{array}{l}\text { Circle } \\
\text { Nanoplate }\end{array}$ & $\begin{array}{l}\lambda \max \text { in } \\
\text { Air }(\mathrm{nm})\end{array}$ & $\begin{array}{l}\lambda \max \text { in } \\
\text { IPA }(\mathrm{nm})\end{array}$ & $\begin{array}{l}\text { Shift } \\
(\mathrm{nm})\end{array}$ & $\begin{array}{l}\text { Sensitivity } \\
(\mathrm{nm} / \text { RIU) }\end{array}$ & FOM & $\begin{array}{l}\text { SEM } \\
\text { Images }\end{array}$ \\
\hline P1 & 684 & 742 & 58 & 152 & 1.61 & \\
\hline P2 & 678 & 750 & 72 & 182 & 2.06 & \\
\hline
\end{tabular}

Table 6.3. LSPR $\lambda_{\max }$ shift data and pertinent experimental environmental sensitivity parameters for dipole resonance of two different circular NPs. 


\subsection{CONCLUSIONS}

We present here a study on the effect of shape on the optical response of individual Au NPs. A strategy has been developed to correlate scattering spectra with SEM and AFM images of each individual NP using etched numbers on glass substrates as markers. The AR of individual NPs was calculated based on dimensions determined by SEM and AFM images. The experimental results showed that $\lambda_{\max }$ increased with increasing aspect ratio increasing for all NPs regardless of their geometric shapes. The $l_{\max }$ was most red-shifted and more sensitive to the AR in the order of triangles $>$ hexagons $>$ circles. The RI sensitivity of a single NP was measured by collecting its LSPR spectrum in air and in isopropanol. The refractive index sensitivity order of NPs is triangle $>$ hexagon $>$ circle. These results provide useful information about the relationship between the shape of an individual NP and its plasmon properties and the sensitivity to changes in the refractive index of the environment. 


\section{CHAPTER VII}

\section{SUMMARY AND FUTURE DIRECTIONS}

\subsection{Summary}

This dissertation described the preparation of new and unique Au nanostructure assemblies using nanoparticle synthesis and thiol self-assembly and ligand placeexchange reactions. Au NSs were regioselectively attached onto Au NPs to form NP/NS dimers and multiparticle core-satellite NP/NS assemblies. The dark-field light scattering spectra of the NP/NS assemblies were collected at the single particle level to gain an understanding of the plasmonic coupling of these asymmetric regioselectively controlled

structures. A strategy was developed to correlate the dark-field light scattering spectra of the nanostructures with the SEM and AFM images of the same structures at the single nanostructure level. The optical properties of coupled NP/NS assemblies have been studied as well as the factors that affect the coupling, including attachment location of the NS on the NP, the size of the NS, and the organic ligands linking the NS and NP. The application of this study to refractive index sensing with different shape NPs has also been performed. 
The key conclusions from this research are:

- $\quad$ The light scattering spectrum of the NP/NS assemblies was found to be strongly dependent on the location of NS attachment. The $\lambda_{\max }$ of the dipolar plasmon mode redshifted by an average of $24 \mathrm{~nm}$ for a single 20-30 nm diameter Au NS coupled to the vertex/edge site as compared to $3 \mathrm{~nm}$ for those coupled to the terrace site of the Au NPs. Simulations using the discrete dipole approximation (DDA) method verified the experimental trends, but with a smaller shift.

- $\quad$ Further studies with multiply attached NSs attached to a Au NP indicate a unique synergistic effect, whereby the polarizing effect of a NS on the NP is positively assisted by the presence of other attached NSs. The attachment of a single NS to the NP side edge results in an average red-shift of $22 \mathrm{~nm}$. The attachment of three NSs attached to the sides results in a red-shift of $23 \mathrm{~nm}$ per attached NS. However, the attachment of four NSs around the NP sides results in a shift of $31 \mathrm{~nm} / \mathrm{NS}$. DDA simulations confirmed the experimental data.

- In contrast to the uniform distribution of NSs, we found that asymmetric distributions of multiple NSs attached to the sides of the NP result in complex, broadened, multi-peaked spectra with large plasmonic shifts. The strongest scattering band of these asymmetric structures appears to be much more strongly red-shifted than in the case of an equivalent (with the same number of NSs) symmetric distribution, which has also been confirmed by DDA simulations. 
- A positive shift in the dipolar plasmon mode of the NP/NS heterodimer relative to the original NP, attributed to coupling along the long NP/NS axis, increased with increasing NS size for those attached on the side of the NP in the order of $9 \pm 2 \mathrm{~nm}, 24 \pm 4$ $\mathrm{nm}$, and $98 \pm 16 \mathrm{~nm}$ for 13,24 , and $51 \mathrm{~nm}$ average diameter NSs, respectively. A look at all of the NS sizes studies, revealed a trend where the red-shift of the LSPR band due to dipolar coupling along the long NP/NS axis increased with increasing NS size up until about a $40 \mathrm{~nm}$ diameter NS, but then saturated for sizes about that. The data can be described by the size dependent decay of the electric field at the NS surface. As the NS size increases, the electric field enhancement extends further from the NS surface, leading to the stronger coupling. At large NS sizes, the distance dependence of the electric field does not change very much.

- $\quad$ As the NS size increased, peaks appeared in the scattering spectrum that were correlated to light polarized along different NP/NS axes with respect to the long NP/NS axis. The long axis dipolar coupling mode was so strongly red-shifted compared to the short axis direction and other directions in between, that those different modes could be distinguished in the scattering spectrum. Only one broadened peak appears with a NS of $\sim 24 \mathrm{~nm}$ diameter and smaller due to a very small difference between the different polarization directions, making them indistinguishable. For larger Au NSs attached, peaks corresponding to different polarization directions are distinguishable.

- For NSs attached to the top terrace of the NPs, the shift in the dipolar plasmon mode was $1 \pm 1 \mathrm{~nm}, 3 \pm 1 \mathrm{~nm}$, and $14 \pm 4 \mathrm{~nm}$ for the 13,24 , and $51 \mathrm{~nm}$ NSs, respectively, and they appeared broad with one main peak. These small shifts are due to the fact that the dipolar mode of the individual NP and NS are parallel to one another and weakly coupled. 
- $\quad$ Single triangular, hexagonal, and circular Au NPs were analyzed by dark-field scattering spectroscopy, SEM, and AFM. The scattering $\lambda_{\max }$ increases linearly with increasing aspect ratio for all NPs with a $\lambda_{\max }$ value and slope that follows the order of triangles $>$ hexagons $>$ circles. DDA simulations and literature comparison provided verification of the trends for some shapes. The refractive index sensitivity order of the different shaped NPs follows the order of triangles $>$ hexagons $>$ circles. The shift upon binding a single Au NS of the same size also follows the same general trend.

- More LSPR modes appear in the spectra of coupled NP/NS dimers or multiparticle assemblies linked by Cys compared to 4-ATP and the red-shift for light polarized along the long axis is larger for Cys compared to 4-ATP. The larger shift is attributed to the shorter linker length of Cys compared to 4-ATP, allowing stronger nearfield coupling, and the large shift for the long-axis dipolar mode (longitudinal mode) leads to the multipeak spectrum since it is easily distinguishable from the dipolar mode perpendicular to the long axis (similar to a transverse mode). There is also a blue-shifted peak at $600 \mathrm{~nm}$ that becomes more intense with the Cys linker due to short length and stronger coupling.

- $\quad$ NS attachment to a triangular NP at an edge site versus a terrace site showed very different coupling strengths for the long axis dipolar mode with a Cys linker due to the shorter distance and stronger coupling. These two sites were indistinguishable, however, when the longer 4-ATP linker was used instead. The shorter linker ligand can have a potential benefit of higher site sensitivity for 3D spatial sensing applications. Also, it appears that the conductive aromatic ring of benzene does not lead to significantly enhanced plasmon coupling relative to the $\mathrm{C} 2$ alkane chain, since the distance effect appeared to dominate. 


\subsection{Future Directions}

Future projects should include studies on more organic linkers, refractive index sensing with $\mathrm{Au} \mathrm{NP/NS}$ assemblies, and other types of metal NP/NS assemblies. We have observed that organic linkers have a large effect on the LSPR spectra of NP/NS assemblies. However, we only compared Cys and 4-ATP molecules, which have different dielectric properties, different lengths, and different polarizability. With so many differences, it is impossible to conclude that the spectral differences come from the different molecular length or molecular structure without simulation data and linker ligands with more similarity and only one variable. It might be the combination of the two or three factors or be dominated by one factor. It will be interesting to study 3aminopropanethiol as a linker. Compared to Cys (2-aminoethanethiol), 3aminopropanethiol has one more carbon in the alkane chain, resulting in a similar interparticle distance $(\sim 1.2 \mathrm{~nm})$ between NS and NP as the 4-ATP linker. The advantage of 3-aminopropanethiol as a linker is that it rules out the different distance when comparing with 4-ATP. It also rules out the structural differences, only focusing on interparticle distance, when comparing to Cys.

In the sensing project, we investigated the RI sensitivity of NPs with different shapes. It would also be interesting to explore the sensing applications of the prepared NP/NS assemblies. Calculations show that nanoparticle coupling can enhance the RI sensitivity. ${ }^{85}$ To the best of our knowledge, there has been no report comparing the RI sensitivity of a single nanostructure before and after coupling with another nanostructure. Not only would the RI affect the LSPR band, but a change in NP/NS distance in the presence of analyte would lead to significant shifts in $\lambda_{\max }$. Finally, the SERS signal can be greatly enhanced by nanoparticle coupling. It would be very interesting to compare 
the SERS enhancement factor between a single NP before and after coupling with a NS, with significant enhancements expected in the coupled structures. Another interesting sensing application of this research would be to use this approach for the detection of biomolecules, including proteins and DNA. The LSPR shift caused by a single protein might be detected by controlling the protein binding to the edge sites of the NP due to the strong electromagnetic filed localized around the sharp edges.

In addition, NSs and NPs of other metals such as Ag or Pt might bring in some other interesting optical phenomenon and applications. For example, Ag nanoparticles are more sensitive than Au nanoparticles in LSPR sensing applications. It will be good to know the shift difference caused by a single Au NP or NS compared to a Ag NS or NP or coupled structures to better understand the main factors controlling LSPR sensors. Pt is a very useful metal in the field of catalysis. A Pt NS attached to a Au NP might serve as a good platform to perform catalysis at the single particle level and monitor the reaction spectroscopically. 


\section{REFERENCES}

1. Brown, L. V.; Sobhani, H.; Lassiter, J. B.; Nordlander, P.; Halas, N. J. ACS Nano 2010, 4,819-832.

2. Pedro, H. C. C.; Claire, M. C.; Matthew, R.; Younan, X. Nanotechnology 2009, 20,434020 .

3. Ozbay, E. Science 2006, 311,189-193.

4. Maier, S. A.; Atwater, H. A. Journal of Applied Physics 2005, 98,011101.

5. Ko, S. H.; Park, I.; Pan, H.; Grigoropoulos, C. P.; Pisano, A. P.; Luscombe, C. K.; Fréchet, J. M. J. Nano Letters 2007, 7,1869-1877.

6. $\quad$ Ko, S. H.; Pan, H.; Grigoropoulos, C. P.; Luscombe, C. K.; Fréchet, J. M. J.; Poulikakos, D. Applied Physics Letters 2007, 90,141103.

7. Haes, A. J.; Chang, L.; Klein, W. L.; Van Duyne, R. P. Journal of the American Chemical Society 2005, 127,2264-2271.

8. $\quad$ Storhoff, J. J.; Elghanian, R.; Mucic, R. C.; Mirkin, C. A.; Letsinger, R. L. Journal of the American Chemical Society 1998, 120,1959-1964.

9. $\quad$ El-Sayed, I. H.; Huang, X.; El-Sayed, M. A. Nano Letters 2005, 5,829-834.

10. Huang, X.; El-Sayed, I. H.; Qian, W.; El-Sayed, M. A. Journal of the American Chemical Society 2006, 128,2115-2120.

11. Lee, K.-S.; El-Sayed, M. A. J. Phys. Chem. B 2006, 110,19220-19225.

12. Kelly, K. L.; Coronado, E.; Zhao, L. L.; Schatz, G. C. The Journal of Physical Chemistry $B$ 2003, 107,668-677.

13. Chen, H. M.; Hsin, C. F.; Liu, R.-S.; Lee, J.-F.; Jang, L.-Y. The Journal of Physical Chemistry C 2007, 111,5909-5914.

14. Murphy, C. J.; Sau, T. K.; Gole, A. M.; Orendorff, C. J.; Gao, J.; Gou, L.;

Hunyadi, S. E.; Li, T. The Journal of Physical Chemistry B 2005, 109, 13857-13870.

15. Chen, J.; Wang, D.; Xi, J.; Au, L.; Siekkinen, A.; Warsen, A.; Li, Z.-Y.; Zhang, H.; Xia, Y.; Li, X. Nano Letters 2007, 7,1318-1322.

16. Koh, A. L.; Bao, K.; Khan, I.; Smith, W. E.; Kothleitner, G.; Nordlander, P.; Maier, S. A.; McComb, D. W. ACS Nano 2009, 3,3015-3022.

17. Pillai, Z. S.; Kamat, P. V. The Journal of Physical Chemistry B 2004, 108,945951.

18. Turkevich, J.; Stevenson, P. C.; Hillier, J. Discussions of the Faraday Society 1951, 11,55-75.

19. Jana, N. R.; Gearheart, L.; Murphy, C. J. The Journal of Physical Chemistry B 2001, 105,4065-4067.

20. Xiong, Y.; Cai, H.; Wiley, B. J.; Wang, J.; Kim, M. J.; Xia, Y. Journal of the American Chemical Society 2007, 129,3665-3675.

21. Beeram, S. R.; Zamborini, F. P. J. Am. Chem. Soc. 2009, 131,11689-11691.

22. Mieszawska, A. J.; Jalilian, R.; Sumanasekera, G. U.; Zamborini, F. P. Journal of the American Chemical Society 2005, 127,10822-10823. 
23. Mieszawska, A. J.; Slawinski, G. W.; Zamborini, F. P. Journal of the American Chemical Society 2006, 128,5622-5623.

24. Skrabalak, S. E.; Wiley, B. J.; Kim, M.; Formo, E. V.; Xia, Y. Nano Letters 2008, 8,2077-2081.

25. Wiley, B. J.; Im, S. H.; Li, Z.-Y.; McLellan, J.; Siekkinen, A.; Xia, Y. The Journal of Physical Chemistry B 2006, 110,15666-15675.

26. Wiley, B.; Herricks, T.; Sun, Y.; Xia, Y. Nano Letters 2004, 4,1733-1739.

27. Sun, Y.; Mayers, B.; Xia, Y. Nano Letters 2003, 3,675-679.

28. Lu, X.; Tuan, H.-Y.; Chen, J.; Li, Z.-Y.; Korgel, B. A.; Xia, Y. Journal of the American Chemical Society 2007, 129,1733-1742.

29. Chen, J.; Wiley, B.; Li, Z. Y.; Campbell, D.; Saeki, F.; Cang, H.; Au, L.; Lee, J.; Li, X.; Xia, Y. Advanced Materials 2005, 17,2255-2261.

30. Sun, Y.; Mayers, B.; Xia, Y. Advanced Materials 2003, 15,641-646.

31. James, J. Z.; Lucas, D.; Koshland, C. P. Environmental science \& technology 2012, 46,9557-9562.

32. Inuta, M.; Arakawa, R.; Kawasaki, H. Analyst 2011, 136,1167-1176.

33. Marinakos, S. M.; Chen, S.; Chilkoti, A. Analytical Chemistry 2007, 79,52785283.

34. Mitsui, K.; Handa, Y.; Kajikawa, K. Applied Physics Letters 2004, 85,4231-4233.

35. Nath, N.; Chilkoti, A. Analytical Chemistry 2002, 74,504-509.

36. Martin, O. J. F. Microelectronic Engineering 2003, 67-68,24-30.

37. Sung, J.; Hicks, E. M.; Van Duyne, R. P.; Spears, K. G. J. Phys. Chem. C 2008, 112,4091-4096.

38. Hicks, E. M.; Zou, S.; Schatz, G. C.; Spears, K. G.; Van Duyne, R. P.;

Gunnarsson, L.; Rindzevicius, T.; Kasemo, B.; Käll, M. Nano Lett. 2005, 5,1065-1070.

39. Lamprecht, B.; Schider, G.; Lechner, R. T.; Ditlbacher, H.; Krenn, J. R.; Leitner, A.; Aussenegg, F. R. Phys. Rev. Lett. 2000, 84,4721-4724.

40. Tong, H. D.; Jansen, H. V.; Gadgil, V. J.; Bostan, C. G.; Berenschot, E.; van Rijn, C. J. M.; Elwenspoek, M. Nano Letters 2004, 4,283-287.

41. Dick, L. A.; McFarland, A. D.; Haynes, C. L.; Van Duyne, R. P. The Journal of Physical Chemistry B 2002, 106,853-860.

42. Hulteen, J. C.; Van Duyne, R. P. Journal of Vacuum Science \&amp; Technology A 1995, 13,1553-1558.

43. Ito, T.; Okazaki, S. Nature 2000, 406,1027-1031.

44. Dhawan, A.; Yan, D.; Fei, Y.; Gerhold, M. D.; Misra, V.; Vo-Dinh, T. Sensors Journal, IEEE 2010, 10,608-616.

45. Haynes, C. L.; Van Duyne, R. P. The Journal of Physical Chemistry B 2001, 105,5599-5611.

46. Guffey, M. J.; Scherer, N. F. Nano Lett. 2010, 10,4302.

47. Urban, A. S.; Lutich, A. A.; Stefani, F. D.; Feldmann, J. Nano Lett. 2010, 10,4794.

48. Kraus, T.; Malaquin, L.; Schmid, H.; Riess, W.; Spencer, N. D.; Wolf, H. Nat. Nanotechnol. 2007, 2,570.

49. Barsotti, J. R. J.; Stellacci, F. J. Mater. Chem. 2006, 16,962-965.

50. Taub, N.; Krichevski, O.; Markovich, G. The Journal of Physical Chemistry B 2003, 107,11579-11582.

51. Zhang, J.; Kambayashi, M.; Oyama, M. Electrochemistry Communications 2004, 6,683-688.

52. Beeram, S. R.; Zamborini, F. P. ACS Nano 2010, 4,3633-3646. 
53. Beeram, S. R.; Zamborini, F. P. The Journal of Physical Chemistry C 2011, 115,7364-7371.

54. Willets, K. A.; Van Duyne, R. P. Annu. Rev. Phys. Chem. 2007, 58,267-297.

55. Kelly, K. L.; Coronado, E.; Zhao, L. L.; Schatz, G. C. J. Phys. Chem. B 2002, $107,668-677$.

56. Link, S.; El-Sayed, M. A. J. Phys. Chem. B 1999, 103,8410-8426.

57. Sagle, L. B.; Ruvuna, L. K.; Ruemmele, J. A.; Van Duyne, R. P. Nanomedicine 2011, 6,1447-1462.

58. Link, S.; El-Sayed, M. A. The Journal of Physical Chemistry B 1999, 103,84108426.

59. Indrasekara, A. S. D. S.; Meyers, S.; Shubeita, S.; Feldman, L. C.; Gustafsson, T.; Fabris, L. Nanoscale 2014, 6,8891-8899.

60. Barbosa, S.; Agrawal, A.; Rodríguez-Lorenzo, L.; Pastoriza-Santos, I.; AlvarezPuebla, R. A.; Kornowski, A.; Weller, H.; Liz-Marzán, L. M. Langmuir 2010, 26,1494314950.

61. Kim, C.; Song, H.-M.; Cai, X.; Yao, J.; Wei, A.; Wang, L. V. Journal of Materials Chemistry 2011, 21,2841-2844.

62. Dam, D. H. M.; Culver, K. S. B.; Odom, T. W. Molecular Pharmaceutics 2014, 11,580-587.

63. Amendola, V.; Bakr, O.; Stellacci, F. Plasmonics 2010, 5,85-97.

64. Mock, J. J.; Barbic, M.; Smith, D. R.; Schultz, D. A.; Schultz, S. J. Chem. Phys. 2002, 116,6755-6759.

65. Aizpurua, J.; Bryant, G. W.; Richter, L. J.; García de Abajo, F. J.; Kelley, B. K.; Mallouk, T. Physical Review B 2005, 71,235420.

66. Sheikholeslami, S.; Jun, Y.-w.; Jain, P. K.; Alivisatos, A. P. Nano Lett. 2010, 10,2655-2660.

67. Aćimović, S. S.; Kreuzer, M. P.; González, M. U.; Quidant, R. ACS Nano 2009, 3,1231-1237.

68. Hall, W. P.; Ngatia, S. N.; Van Duyne, R. P. J. Phys. Chem. C 2011, 115,14101414.

69. Sonnichsen, C.; Reinhard, B. M.; Liphardt, J.; Alivisatos, A. P. Nat Biotechnol 2005, 23,741-745.

70. $\quad$ Kneipp, K.; Wang, Y.; Kneipp, H.; Perelman, L. T.; Itzkan, I.; Dasari, R. R.; Feld, M. S. Phys. Rev. Lett. 1997, 78,1667-1670.

71. Nie, S.; Emory, S. R. Science 1997, 275,1102-1106.

72. Michaels, A. M.; Jiang; Brus, L. J. Phys. Chem. B 2000, 104,11965-11971.

73. Camden, J. P.; Dieringer, J. A.; Wang, Y.; Masiello, D. J.; Marks, L. D.; Schatz, G. C.; Van Duyne, R. P. J. Am. Chem. Soc. 2008, 130,12616-12617.

74. Talley, C. E.; Jackson, J. B.; Oubre, C.; Grady, N. K.; Hollars, C. W.; Lane, S. M.; Huser, T. R.; Nordlander, P.; Halas, N. J. Nano Lett. 2005, 5,1569-1574.

75. Maier, S. A. Nat. Photonics 2008, 2,460-461.

76. Solis, D.; Paul, A.; Olson, J.; Slaughter, L. S.; Swanglap, P.; Chang, W.-S.; Link, S. nano Lett. 2013, 13,4779-4784.

77. Halas, N. J.; Lal, S.; Chang, W.-S.; Link, S.; Nordlander, P. Chemical Reviews 2011, 111,3913-3961.

78. Sheikholeslami, S.; Jun, Y.-w.; Jain, P. K.; Alivisatos, A. P. Nano Letters 2010, 10,2655-2660.

79. Prodan, E.; Radloff, C.; Halas, N. J.; Nordlander, P. Science 2003, 302,419-422. 
80. Jain, P. K.; Eustis, S.; El-Sayed, M. A. J. Phys. Chem. B 2006, 110,18243-18253. 81. Nordlander, P.; Oubre, C.; Prodan, E.; Li, K.; Stockman, M. I. Nano Lett. 2004, 4,899-903.

82. Khoury, C. G.; Norton, S. J.; Vo-Dinh, T. ACS Nano 2009, 3,2776-2788.

83. Kim, D.-S.; Heo, J.; Ahn, S.-H.; Han, S. W.; Yun, W. S.; Kim, Z. H. Nano Lett. 2009, 9,3619-3625.

84. Lassiter, J. B.; Aizpurua, J.; Hernandez, L. I.; Brandl, D. W.; Romero, I.; Lal, S.; Hafner, J. H.; Nordlander, P.; Halas, N. J. Nano Lett. 2008, 8, 1212-1218.

85. Jain, P. K.; Huang, W.; El-Sayed, M. A. Nano Lett. 2007, 7,2080-2088.

86. Funston, A. M.; Novo, C.; Davis, T. J.; Mulvaney, P. Nano Letters 2009, 9,16511658.

87. Koh, A. L.; Fernández-Dom.nguez, A. I.; McComb, D. W.; Maier, S. A.; Yang, J. K. W. Nano Letters 2011, 11,1323-1330.

88. Duan, H.; Fernández-Domínguez, A. I.; Bosman, M.; Maier, S. A.; Yang, J. K. W. Nano Letters 2012, 12,1683-1689.

89. Sannomiya, T.; Hafner, C.; Voros, J. Nano Letters 2008, 8,3450-3455.

90. Xia X, X. Y. R. M. Q. D. J. Mater. Chem. C 2013, 1,6145-6150.

91. Sebba, D. S.; Mock, J. J.; Smith, D. R.; LaBean, T. H.; Lazarides, A. A. Nano Lett. 2008, 8,1803-1808.

92. Gandra, N.; Abbas, A.; Tian, L.; Singamaneni, S. Nano Lett. 2012, 12,2645-2651.

93. $\mathrm{Xu}, \mathrm{L} . ;$ Kuang, H.; Xu, C.; Ma, W.; Wang, L.; Kotov, N. A. J. Am. Chem. Soc. 2011, 134,1699-1709.

94. Yoon, J. H.; Zhou, Y.; Blaber, M. G.; Schatz, G. C.; Yoon, S. The Journal of Physical Chemistry Letters 2013, 4,1371-1378.

95. Ross, B. M. W. J. R. W. T. L. L. P. Appl. Phys. Lett. 2009, 95,193112.

96. Yang, W. H.; Schatz, G. C.; Van Duyne, R. P. The Journal of Chemical Physics 1995, 103,869-875.

97. Kane, Y. Antennas and Propagation, IEEE Transactions on 1966, 14,302-307.

98. Purcell, E. M.; Pennypacker, C. R. Astrophysical Journal 1973, 186.

99. Draine, B. T. Astrophysical Journal 1988, 333.

100. Jun, Y.-w.; Sheikholeslami, S.; Hostetter, D. R.; Tajon, C.; Craik, C. S.;

Alivisatos, A. P. P. Natl. Acad. Sci. USA 2009, 106,17735-17740.

101. Sonnichsen, C.; Reinhard, B. M.; Liphardt, J.; Alivisatos, A. P. Nat. Biotech. 2005, 23,741-745.

102. Charles, D. E.; Aherne, D.; Gara, M.; Ledwith, D. M.; Gun'ko, Y. K.; Kelly, J. M.; Blau, W. J.; Brennan-Fournet, M. E. ACS Nano 2009, 4,55-64.

103. Chen, H.; Kou, X.; Yang, Z.; Ni, W.; Wang, J. Langmuir 2008, 24,5233-5237.

104. Sherry, L. J.; Chang, S.-H.; Schatz, G. C.; Van Duyne, R. P.; Wiley, B. J.; Xia, Y. Nano Lett 2005, 5,2034-2038.

105. Ghosh, S. K.; Nath, S.; Kundu, S.; Esumi, K.; Pal, T. The Journal of Physical Chemistry B 2004, 108,13963-13971.

106. Chen, H.; Kou, X.; Yang, Z.; Ni, W.; Wang, J. Langmuir 2008, 24,5233-5237.

107. Hanarp, P.; Käll, M.; Sutherland, D. S. The Journal of Physical Chemistry B 2003, 107,5768-5772.

108. Jensen, T. R.; Duval, M. L.; Kelly, K. L.; Lazarides, A. A.; Schatz, G. C.; Van Duyne, R. P. J. Phys. Chem. B 1999, 103,9846-9853.

109. Larsson, E. M.; Alegret, J.; Käll, M.; Sutherland, D. S. Nano Letters 2007, 7,1256-1263. 
110. Sönnichsen, C.; Geier, S.; Hecker, N. E.; von Plessen, G.; Feldmann, J.; Ditlbacher, H.; Lamprecht, B.; Krenn, J. R.; Aussenegg, F. R.; Chan, V. Z.-H.; Spatz, J. P.; Möller, M. Applied Physics Letters 2000, 77,2949-2951.

111. Schultz, S.; Smith, D. R.; Mock, J. J.; Schultz, D. A. Proceedings of the National Academy of Sciences 2000, 97,996-1001.

112. McFarland, A. D.; Van Duyne, R. P. Nano Letters 2003, 3, 1057-1062.

113. Murray, W. A.; Auguié, B.; Barnes, W. L. J. Phys. Chem. C 2009, 113,5120-5125.

114. Yu, C.; Irudayaraj, J. Biophysical Journal 93,3684-3692.

115. Frederix, F.; Friedt, J.-M.; Choi, K.-H.; Laureyn, W.; Campitelli, A.; Mondelaers, D.; Maes, G.; Borghs, G. Analytical Chemistry 2003, 75,6894-6900.

116. Yonzon, C. R.; Jeoung, E.; Zou, S.; Schatz, G. C.; Mrksich, M.; Van Duyne, R. P. Journal of the American Chemical Society 2004, 126,12669-12676.

117. Raschke, G.; Kowarik, S.; Franzl, T.; Sönnichsen, C.; Klar, T. A.; Feldmann, J.; Nichtl, A.; Kürzinger, K. Nano Letters 2003, 3,935-938.

118. Raschke, G.; Brogl, S.; Susha, A. S.; Rogach, A. L.; Klar, T. A.; Feldmann, J.; Fieres, B.; Petkov, N.; Bein, T.; Nichtl, A.; Kürzinger, K. Nano Letters 2004, 4,18531857.

119. Nusz, G. J.; Marinakos, S. M.; Curry, A. C.; Dahlin, A.; Höök, F.; Wax, A.; Chilkoti, A. Analytical Chemistry 2008, 80,984-989.

120. Becker, J.; Trügler, A.; Jakab, A.; Hohenester, U.; Sönnichsen, C. Plasmonics 2010, 5,161-167.

121. Baciu, C. L.; Becker, J.; Janshoff, A.; Sönnichsen, C. Nano Letters 2008, 8,17241728 .

122. Unger, A.; Kreiter, M. The Journal of Physical Chemistry C 2009, 113,1224312251.

123. Davis, T. J.; Vernon, K. C.; Gómez, D. E. Journal of Applied Physics 2009, 106,043502 .

124. Mayer, K. M.; Hao, F.; Lee, S.; Nordlander, P.; Hafner, J. H. Nanotechnology 2010, 21.

125. Liu, X.; Xu, H.; Xia, H.; Wang, D. Langmuir 2012, 28,13720-13726.

126. Nehl, C. L.; Hafner, J. H. J. Mater. Chem. 2008, 18,2415-2419.

127. Mulvihill, M. J.; Ling, X. Y.; Henzie, J.; Yang, P. J. Am. Chem. Soc. 2009, $132,268-274$.

128. El Khoury, J. M.; Zhou, X.; Qu, L.; Dai, L.; Urbas, A.; Li, Q. Chemical Communications 2009,2109-2111.

129. Jain, P. K.; Deeb, C. Handbook of Molecular Plasmonics 2013,261-294.

130. Wang, H.; Brandl, D. W.; Nordlander, P.; Halas, N. J. Acc. Chem. Res. 2007, 40,53-62.

131. Hao, E.; Schatz, G. C. J. Chem. Phys. 2004, 120,357-366.

132. Prodan, E.; Radloff, C.; Halas, N. J.; Nordlander, P. Science 2003, 302,419-422.

133. Brown, L. V.; Sobhani, H.; Lassiter, J. B.; Nordlander, P.; Halas, N. J. ACS Nano 2010, 4,819-832.

134. Liu, N.; Hentschel, M.; Weiss, T.; Alivisatos, A. P.; Giessen, H. Science 2011, 332,1407-1410.

135. Ross, B. M.; Waldeisen, J. R.; Wang, T.; Lee, L. P. Appl. Phys. Lett. 2009, 95, 193112.

136. Yoon, J. H.; Zhou, Y.; Blaber, M. G.; Schatz, G. C.; Yoon, S. J. Phys. Chem. Lett. 2013, 4,1371-1378. 
137. Pierrat, S.; Zins, I.; Breivogel, A.; Sönnichsen, C. Nano Lett. 2006, 7,259-263.

138. Hostetler, M. J.; Templeton, A. C.; Murray, R. W. Langmuir 1999, 15,3782-3789.

139. Takao, O.; Katsuhiro, I.; Satoko, N.; Kazushi, M. Applied Physics Express 2014, 7,065001 .

140. Gabudean, A. M.; Biro, D.; Astilean, S. Journal of Molecular Structure 2011, $993,420-424$.

141. Johnson, P. B.; Christy, R. W. Physical Review B 1972, 6,4370-4379.

142. Beeram, S. R.; Zamborini, F. P. J. Phys. Chem. C 2011, 115,7364-7371.

143. Stroyer-Hansen, T.; Svendsen, E. N. J. Mol. Struct. 1997, 410-411,349-352.

144. Hinchliffe, A.; Mkadmh, A.; Nikolaidi, B.; Soscún, H.; Abu-Awwad, F. Central

Eur. J. Chem. 2006, 4,743-759.

145. Hostetler, M. J.; Templeton, A. C.; Murray, R. W. Langmuir 1999, 15,3782-3789.

146. Fang, A.; White, S.; Jain, P. K.; Zamborini, F. P. Nano Lett 2014, 15,542-548.

147. Yoon, J. H.; Lim, J.; Yoon, S. ACS Nano 2012, 6,7199-7208.

148. Novak, J. P.; Feldheim, D. L. Journal of the American Chemical Society 2000, 122,3979-3980.

149. Yoon, J. H.; Yoon, S. Langmuir 2013, 29,14772-14778.

150. Dadosh, T.; Gordin, Y.; Krahne, R.; Khivrich, I.; Mahalu, D.; Frydman, V.; Sperling, J.; Yacoby, A.; Bar-Joseph, I. Nature 2005, 436,677-680.

151. Jensen, T.; Kelly, L.; Lazarides, A.; Schatz, G. Journal of Cluster Science 1999, 10,295-317.

152. Hill, R. T.; Mock, J. J.; Hucknall, A.; Wolter, S. D.; Jokerst, N. M.; Smith, D. R.; Chilkoti, A. ACS Nano 2012, 6,9237-9246.

153. Su, K. H.; Wei, Q. H.; Zhang, X.; Mock, J. J.; Smith, D. R.; Schultz, S. Nano Lett 2003, 3,1087-1090.

154. Wang, L.; Zhu, Y.; Xu, L.; Chen, W.; Kuang, H.; Liu, L.; Agarwal, A.; Xu, C.; Kotov, N. A. Angew. Chem. Int. Ed. 2010, 49,5472-5475.

155. McFarland, A. D.; Van Duyne, R. P. Nano Lett 2003, 3,1057-1062.

156. Shuford, K. L.; Ratner, M. A.; Schatz, G. C. J. Chem. Phys 2005, 123,114713. 


\section{APPENDIX}

Chapter III was reproduced with permission from the American Chemical Society, Copyright 2015. The simulation work described in that chapter was performed by Sarah White, as part of her $\mathrm{PhD}$ dissertation, and Prashant Jain at the University of

Illinois. Permissions from these co-authors to reproduce their results here have been received.

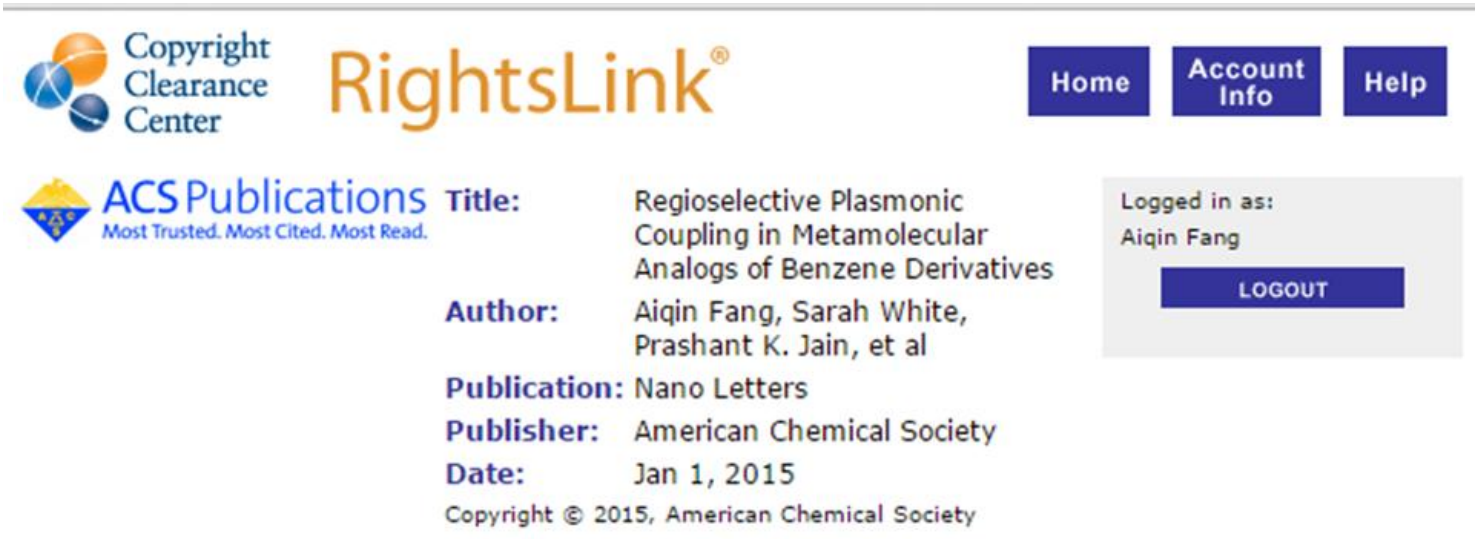

\section{PERMISSION/LICENSE IS GRANTED FOR YOUR ORDER AT NO CHARGE}

This type of permission/license, instead of the standard Terms \& Conditions, is sent to you because no fee is being charged for your order. Please note the following:

- Permission is granted for your request in both print and electronic formats, and translations.

- If figures and/or tables were requested, they may be adapted or used in part.

- Please print this page for your records and send a copy of it to your publisher/graduate school.

- Appropriate credit for the requested material should be given as follows: "Reprinted (adapted) with permission from (COMPLETE REFERENCE CITATION). Copyright (YEAR) American Chemical Society." Insert appropriate information in place of the capitalized words.

- One-time permission is granted only for the use specified in your request. No additional uses are granted (such as derivative works or other editions). For any other uses, please submit a new request. 


\title{
CURRICULUM VITAE
}

\author{
Aiqin Fang \\ Department of Chemistry \\ University of Louisville \\ Louisville, KY 40292 \\ Mobile: (502) 294-0866 \\ E-mail: aiqin fang@yahoo.com
}

\section{EDUCATIONAL BACKGROUND:}

$>\quad$ University of Louisville (Louisville, KY)

2011-present

$\mathrm{Ph} . \mathrm{D}$ in chemistry, expected May 2015

Department of Chemistry

$>\quad$ University of Louisville (Louisville, KY)

2008-2011

MS in chemistry, July 2011

Department of Chemistry

$>\quad$ Ocean University of China (Qingdao, China)

2004-2007

MS in chemistry, July 2007

Department of Marine Drug and Food Analysis

Jinan University (Jinan, China)

2000-2004

Bachelors in Chemistry, July 2004

\section{RESEARCH EXPERIENCE:}

University of Louisville (Louisville, KY)

2005-2011

$>\quad$ Quantitating location and shape effect of nanostructures on Localized Surface Plasmon Resonance (LSPR) shifts.

$>\quad$ Simultaneous conductivity and SERS measurement of polyaniline film at electrochemically fabricated nanojunctions.

$>\quad$ SERS single molecule detection at electrochemically fabricated silver nanowire and nanoparticle junctions.

$>\quad$ Single protein level detection of human serum anti-immunoglobulin G by LSPR and AFM.

$>\quad$ Comprehensive metabolomic analysis of rat plasma using GCxGC TOFMS and 653 metabolites were identified including 45 acids, 70 alcohols and 72 esters. 
Metabolomic analysis of wheat (normal vs disease) using GCxGC TOFMS, 4 metabolites were found up-regulated and serve as potential biomarkers.

$>\quad$ Repeatability study of 53 metabolites using GCxGC TOFMS with nonpolar by polar column combination.

Determination of monosaccharide composition in traditional Chinese medicine rhizoma phramitis and typhaceae polysaccharides by HPCE

\section{RESEARCH JOURNAL PUBLICATIONS:}

$>\quad$ Fang, A.; White, S.; Jain, P. K.; Zamborini, F. P. "Regio-selective Plasmonic Coupling in Metamolecular Analogs of Benzene Derivatives". Nano Letters. 2015, 15, 542

Wang, B.; Fang, A.; Shi, X.; Kim, S. H.; Zhang, X. "DISCO2: a comprehensive peak alignment algorithm for two-dimensional gas chromatography time-of-flight mass spectrometry". Bio-Inspired Computing and Applications, 2012, 6840, 486.

$>\quad$ Zhao, Y.; Zhang, J.; Wang, B.; Kim, S. H.; Fang, A.; Bogdanov, B.; Zhou, Z.; McClain, C.; Zhang, X. "A method of calculating the second dimension retention index in comprehensive two-dimensional gas chromatography time-of-flight mass spectrometry". Journal of chromatography A, 2011, 1218, 2577.

$>\quad$ Zhang, J.; Fang, A.; Wang, B.; Bogdanov, B.; Kim, S. H.; Zhou, Z.; McClain, C.; Zhang, X. "iMatch: A retention index tool for analysis of gas chromatography-mass spectrometry data". Journal of chromatography A, 2011, 1218, 6522.

$>\quad$ Kim, S.; Koo, I.; Fang, A.; Zhang, X. "Smith-Waterman peak alignment for comprehensive two-dimensional gas chromatography-mass spectrometry" BMC bioinformatics, 2011, 12, 235.

$>\quad$ Kim, S.; Fang, A.; Wang, B.; Jeong, J.; Zhang, X. “An optimal peak alignment for comprehensive two-dimensional gas chromatography mass spectrometry using mixture similarity measure", Bioinformatics, 2011, 27, 1660.

$>\quad$ Wang, B.; Fang, A.; Heim, J.; Bogdanov, B.; Pugh, S.; Libardoni, M.; Zhang, X "DISCO: Distance and Spectrum Correlation Optimization Alignment for TwoDimensional Gas Chromatography Time-of-Flight Mass Spectrometry-Based Metabolomics". Analytical Chemistry, 2010, 82, 5069.

$>\quad$ Zhang, X.; Fang, A.; Riley, C. P.; Wang, M.; Regnier, F. E. Buck, C. "Multidimensional liquid chromatography in proteomics - A review". Analytica Chimica Acta, 2010, 664, 101.

\section{PRESENTATIONS:}

Zamborini, F. P.; Fang, A. "Plasmonic nanoparticle counter" Oral presentation at FACSS SCIX. September 2014, Reno, NV.

$>$ Fang, A.; Zamborini, F. P. "Plasmonic counter of nanoparticle". Poster presentation at Gordon Research Conference on Noble Metal Nanoparticles. June 2014, Mount Holyoke College, South Hadley, MA. 
Zamborini, F. P.; Fang, A.; Shah, N. "The combination of resistance and spectroscopic measurements for analytical measurements with metallic nanostructures" Oral presentation at Pittcon Conference. March 2014, Chicago, IL.

$>\quad$ Zamborini, F. P.; Fang, A.; Shah, N. "Combined electronic and Raman scattering measurements at nanoscale junctions fabricated by electrodeposition of silver nanowires" Oral presentation at American Chemical Society. September 2013, Indianapolis, IN.

\section{INSTRUMENTATION:}

Electrochemistry- Cyclic Voltammetry, Chronocoulorometry and Chronoamperometry

$>\quad$ Microscopic Techniques - Atomic Force Microscopy (AFM), Scanning Electron Microscopy (SEM), Dark Field Microscopy

$>\quad$ Spectroscopy Techniques - UV-vis, Raman, Rayleigh Scattering Spectroscopy, Fourier Transform Infrared Spectroscopy (FT-IR)

$>\quad$ Separation Techniques -HPLC-UV, GC-MS, HPCE-UV.

\section{TEACHING EXPERIENCE:}

$>\quad$ Graduate Teaching Assistant

Fall 2011 - Spring 2014

Proctored and graded for General Chemistry 201/202 and 101/105

Lectured for Chem 201 recitation class

$>\quad$ Graduate Research Mentor:

Taught basic laboratory techniques, instrumentation, synthesis of metal nanostructures and characterization

\section{AWARDS:}

$>\quad$ Graduate student fellowship of chemistry department of University of Louisville

\section{REFERENCES:}

Dr. Francis P. Zamborini (PhD)

Department of Chemistry

University of Louisville

2320 South Brook Street

Louisville, KY 40292

Phone: (502) 852-6550

Email: f.zamborini@louisville.edu 
Dr. Craig A. Grapperhaus (PhD)

Department of Chemistry University of Louisville 2320 South Brook Street Louisville, KY 40292

Phone: (502) 852-5932

Email: grapperhaus@1ouisville.edu

Dr. Marta C. Yappert (PhD)

Department of Chemistry University of Louisville 2320 South Brook Street Louisville, KY 40292

Phone: (502) 8527061

Email: mcyappert@1ouisville.edu 